\title{
WestVirginiaUniversity
}

THE RESEARCH REPOSITORY @ WVU

Graduate Theses, Dissertations, and Problem Reports

2016

\section{Distal Determinants of Cardiovascular Disease Risk Factors}

\author{
Amna Umer
}

Follow this and additional works at: https://researchrepository.wvu.edu/etd

\section{Recommended Citation}

Umer, Amna, "Distal Determinants of Cardiovascular Disease Risk Factors" (2016). Graduate Theses, Dissertations, and Problem Reports. 6848.

https://researchrepository.wvu.edu/etd/6848

This Dissertation is protected by copyright and/or related rights. It has been brought to you by the The Research Repository @ WVU with permission from the rights-holder(s). You are free to use this Dissertation in any way that is permitted by the copyright and related rights legislation that applies to your use. For other uses you must obtain permission from the rights-holder(s) directly, unless additional rights are indicated by a Creative Commons license in the record and/ or on the work itself. This Dissertation has been accepted for inclusion in WVU Graduate Theses, Dissertations, and Problem Reports collection by an authorized administrator of The Research Repository @ WVU.

For more information, please contact researchrepository@mail.wvu.edu. 
Distal Determinants of Cardiovascular Disease Risk Factors

Amna Umer, BDS, MPH

\title{
Dissertation submitted
}

to the School of Public Health

at West Virginia University

in partial fulfillment of the requirements for the degree of

Doctor of Philosophy in

Epidemiology

\author{
Christa L. Lilly, Ph.D., Chair \\ Lesley Cottrell, Ph.D. \\ Peter Giacobbi, Jr., Ph.D. \\ Kim Innes, MSPH, Ph.D. \\ George A. Kelley, DA, FACSM.
}

Department of Epidemiology

Morgantown, West Virginia

2016

Keywords: birth weight, breastfeeding, obesity, cardiovascular disease, blood pressure, and cholesterol

Copyright 2016. Amna Umer, BDS, MPH 


\section{ABSTRACT \\ Distal Determinants of Cardiovascular Disease Risk Factors}

Amna Umer

Introduction: Cardiovascular disease (CVD) is the leading cause of morbidity and mortality in the world. West Virginia (WV) has one of the highest prevalence of CVD in the United States. The first two studies examined the association between perinatal risk factors (birth weight (BTW) and breastfeeding) and subsequent childhood and maternal CVD risk factors 11 years postpartum. The purpose of the third study was to conduct a systematic review and meta-analysis to examine the evidence regarding the relationship between childhood obesity and adult CVD risk factors.

Method: We used longitudinally linked data from three cross-sectional datasets in WV for the first $(\mathrm{N}=19,583)$ and second study $(\mathrm{N}=10,457)$. The outcome variables included blood pressure for children and lipid levels for both mothers and children. The exposures were BTW of the infant (study 1) and reported history of breastfeeding obtained retrospectively when the child was in fifth grade (study 2). Mean differences, correlations, and simple regression analyses were performed to examine the unadjusted associations. Multiple regression analysis was performed adjusting for current body mass index (BMI) and additional covariates. For the third study, the search strategy included (1) electronic searches in multiple databases (PubMed (MEDLINE), Web of Science, and Scopus) on June 5, 2015, and (2) citation tracking ( $\mathrm{N}=4,840$ citations). Studies were included if they met the following criteria (1) longitudinal study-design, (2) childhood exposure and adult outcomes collected on the same individual over time, (3) childhood obesity, as defined by the authors, (4) English language articles, (5) studies published by June, 2015, (6) the primary outcome measures included: systolic blood pressure (SBP), diastolic blood pressure (DBP), total cholesterol (TC), high-density lipoprotein cholesterol (HDL), low-density lipoprotein cholesterol (LDL), non-high-density lipoprotein cholesterol (non-HDL), triglycerides (TG), and (7) outcome not self-reported.

Results: BTW was significantly associated with HDL $(b=0.14 \mathrm{mg} / \mathrm{dL} ; 95 \%$ CI: $0.11,0.18)$, LDL $(b=-0.1 \mathrm{mg} / \mathrm{dL} ; 95 \% \mathrm{CI}:-0.19,-0.016)$, non-HDL $(b=-0.18 \mathrm{mg} / \mathrm{dL} ; 95 \% \mathrm{CI}:-0.28,-0.09)$, and $\log$-TGs $(b=-0.007(-0.008,-0.005)$ per $1000 \mathrm{~g}$ increase in BTW in the adjusted analyses. There was a positive association between BTW and maternal TC levels, which became non-significant in the adjusted analysis $[b=0.4 \mathrm{mg} / \mathrm{dL}(95 \% \mathrm{CI}$ : $-0.01,0.90)$ per $1000 \mathrm{~g}$ increase in BTW]. None of the other maternal lipids were significant in the unadjusted or the adjusted analysis. For the second study, there was a significant association between reported history of breastfeeding and child's TGs (beta $=-0.04 ; 95 \%$ CI: $-0.06,-0.01$ ) when adjusted for the child's current BMI and additional covariates. Maternal lipids were not significantly related to their breastfeeding history. For the third study, a total of 23 studies were included in the systematic review and 21 in the meta-analysis. The findings suggest that childhood obesity is significantly and positively associated with adult SBP $(\mathrm{Zr}=0.11 ; 95 \% \mathrm{CI}: 0.07,0.14)$, DBP $(\mathrm{Zr}=0.11 ; 95 \% \mathrm{CI}: 0.07,0.14)$, and TG ( $\mathrm{Zr}=0.08 ; 95 \% \mathrm{CI}: 0.03,0.13)$, and inversely associated with adult $\mathrm{HDL}(\mathrm{Zr}=-0.06 ; 95 \%$ CI: -0.10, -0.02). For those studies that adjusted for adult BMI, associations were reversed.

Conclusion: Low BTW was associated with poor lipid levels (LDL, HDL, non-HDL, and TG) and breastfeeding was protective for TGs in fifth grade children independent of their current BMI. As CVD risk factors persist from childhood into adulthood, the small effect sizes observed in the first two studies can have potential unfavorable consequence on lipid levels in later adulthood. The results of the systematic review with meta-analysis suggest that childhood obesity is significantly and positively associated with adult SBP, DBP, and TG and negatively associated with adult HDL. Well-designed, longitudinal studies with improved reporting as well as data analysis that include both unadjusted and adjusted associations for adult adiposity are needed before any definitive conclusions can be made. 


\section{DEDICATION}

I dedicate my doctoral dissertation to my parents and my husband. Thank you for your love, support, and encouragement throughout my life.

\section{ACKNOWLEDGEMENTS}

I would like to express sincere gratitude to my dissertation committee chair Dr. Christa Lilly for her constant guidance, support, and friendship without which this work would not have been possible. I would like to thank all my committee members Dr. Lesley Cottrell, Dr. Peter Giacobbi, Dr. Kim Innes, and Dr. George Kelley for their invaluable advice and support. I would specially like to thank Dr. George Kelley for teaching me the complex science of conducting systematic reviews and meta-analysis; Dr. Collin John, Candice Hamilton, and Cris Britton for not only providing me with the data for my dissertation but for their incredible support and encouragement for the past four years; the faculty and staff at the School of Public Health, the WATCH/Birth Score Project team, and the CARDIAC Project team at West Virginia University; Dr. Roger Edwards from Northeastern University for mentoring me on my first research project; my family and friends who provided unconditional love and support throughout this journey. Lastly, I wish to thank my husband who has been my biggest cheerleader and supporter throughout this process. 


\section{Table of Contents}

CHAPTER 1: Project overview ................................................................................................. 1

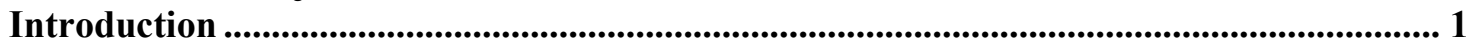

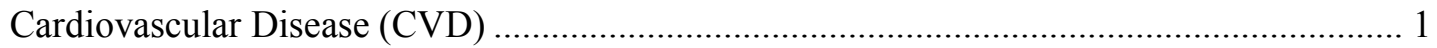

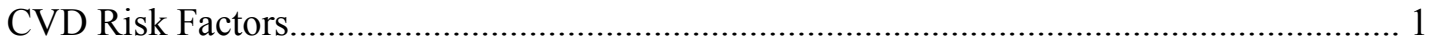

Early Life Exposure and Origins of Childhood and Maternal CVD Risk Factors .................. 5

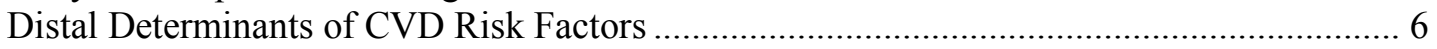

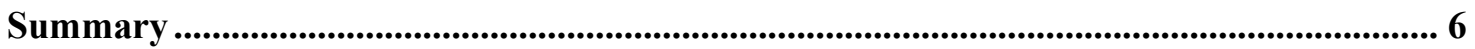

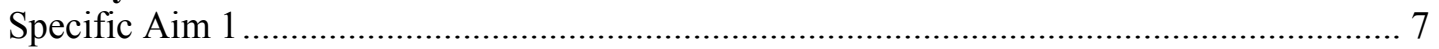

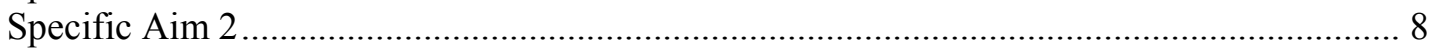

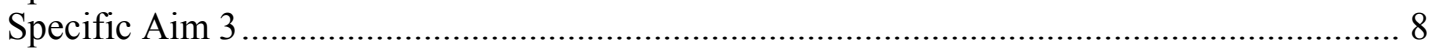

CHAPTER 2: Birth weight and childhood and maternal cardiovascular disease risk

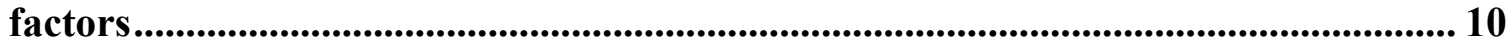

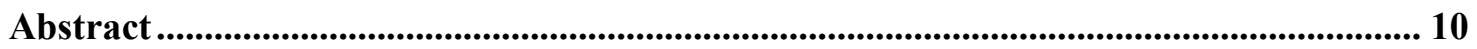

Introduction ...................................................................................................................................................... 11

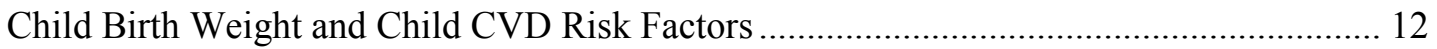

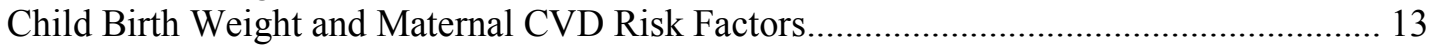

Low Birth Weight, Preterm Births, and Small for Gestational Age ................................... 14

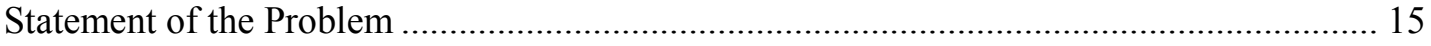

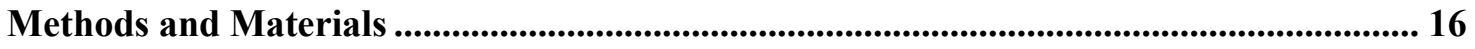

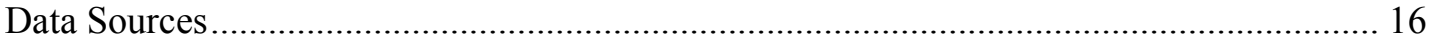

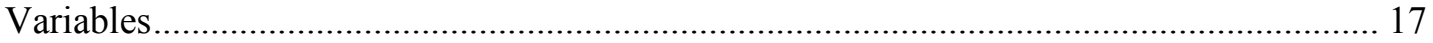

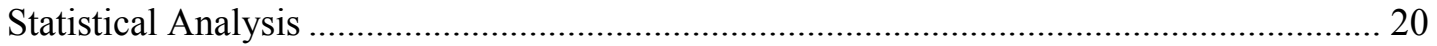

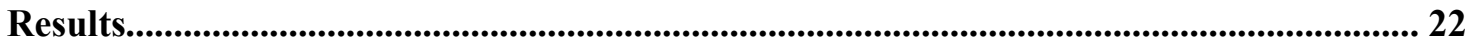

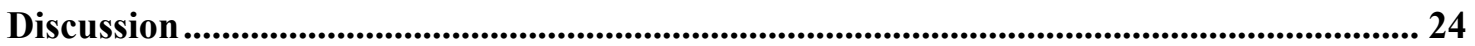

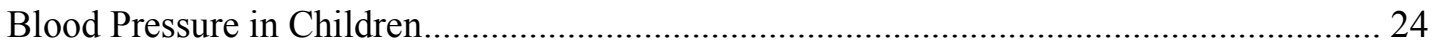

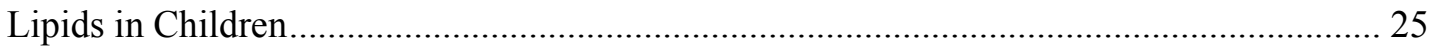

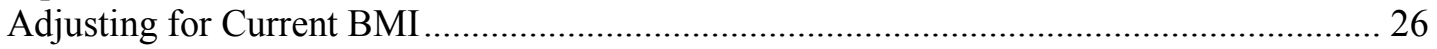

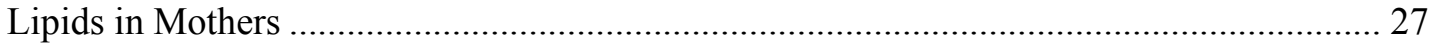

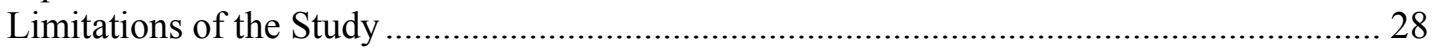

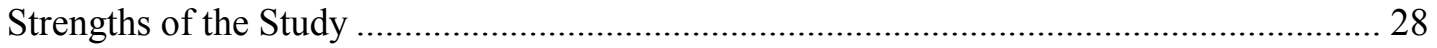

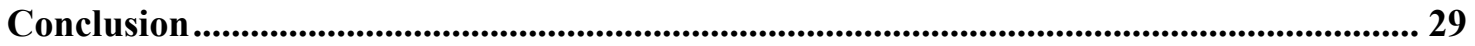

Chapter 3: Breastfeeding and childhood and maternal cardiovascular risk factors 30

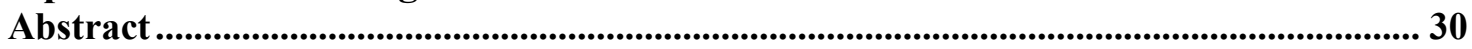

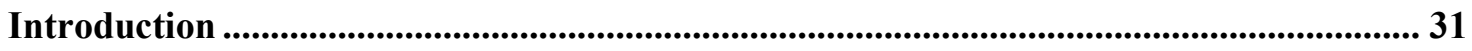

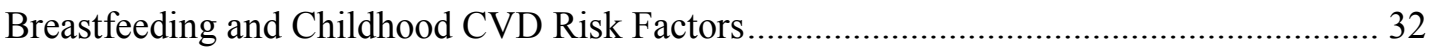

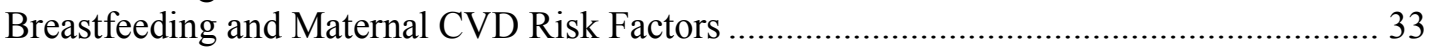

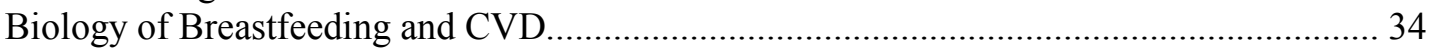

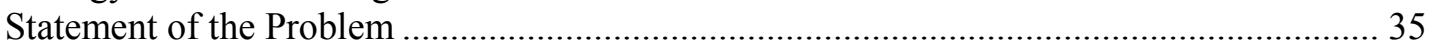

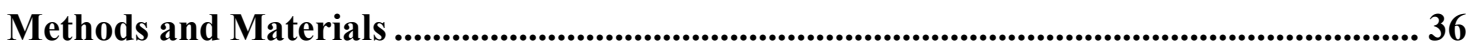

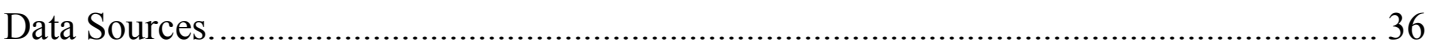

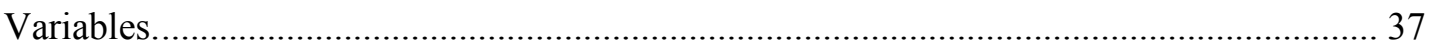

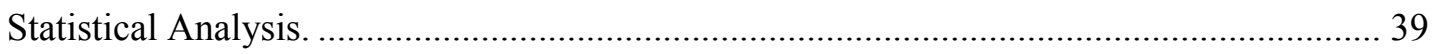

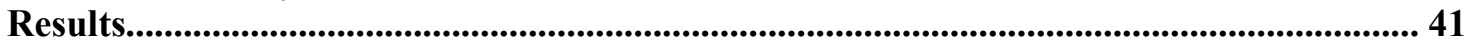

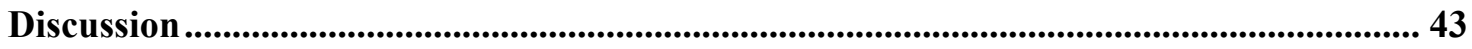

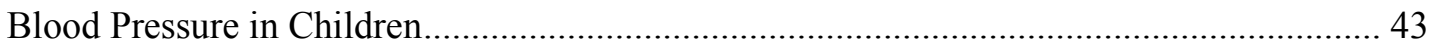

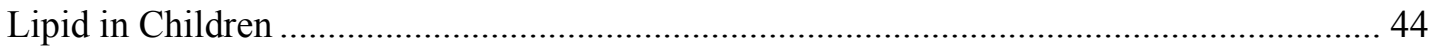

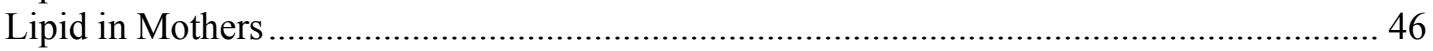

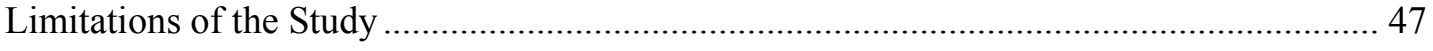




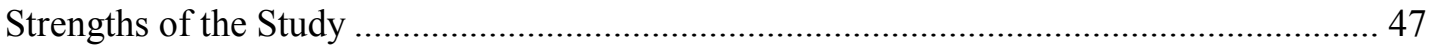

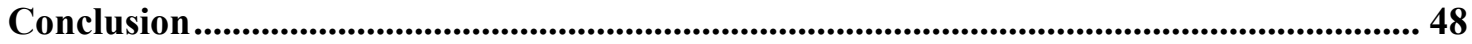

\section{Chapter 4: Childhood obesity and adult cardiovascular disease risk factors: a} systematic review with meta-analysis.......................................................................... 49

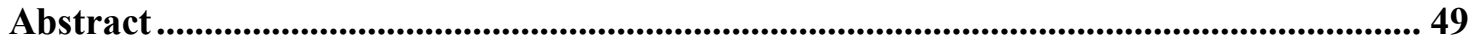

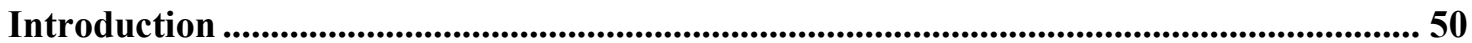

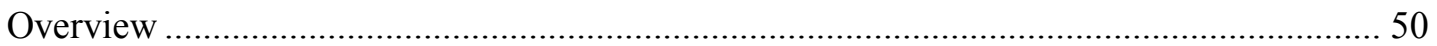

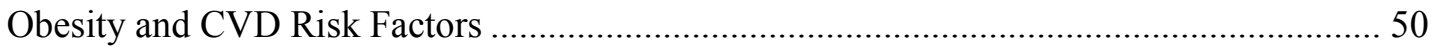

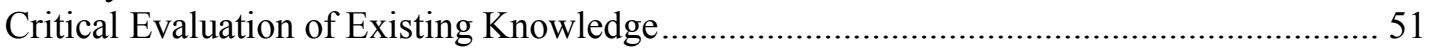

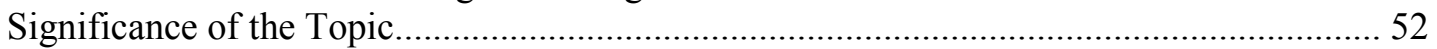

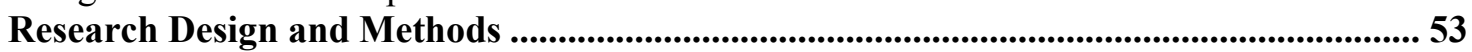

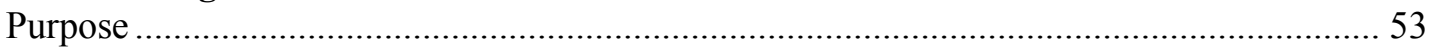

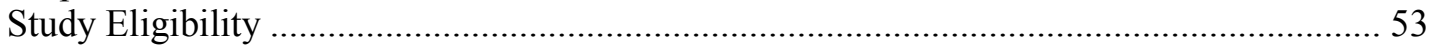

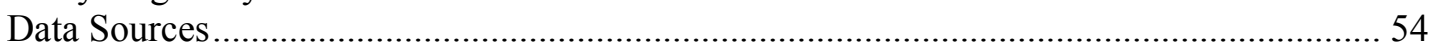

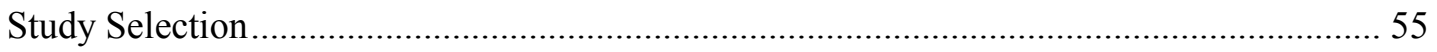

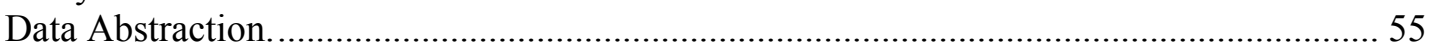

Risk of Bias Assessment ................................................................................ 55

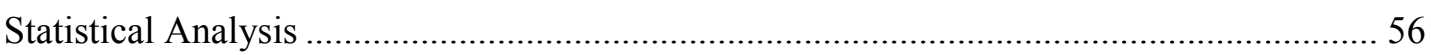

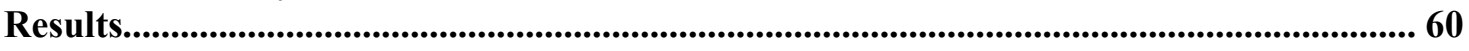

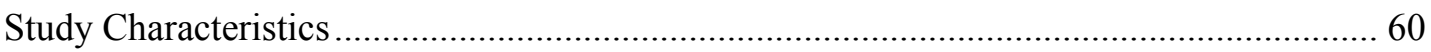

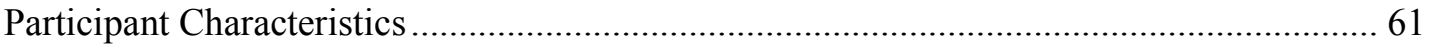

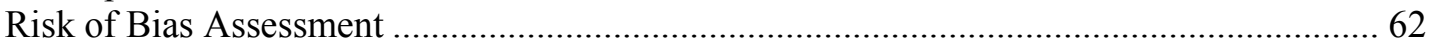

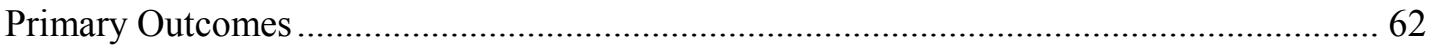

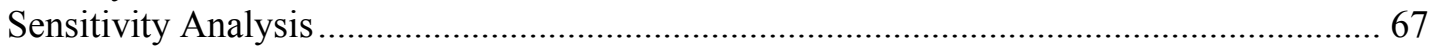

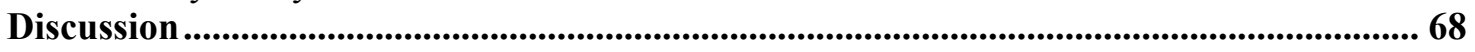

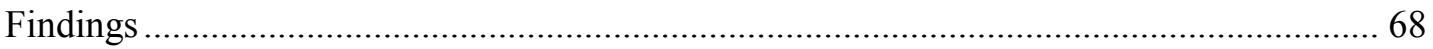

Critical Evaluation of Results compared to Previous Systematic Reviews and Meta-analysis

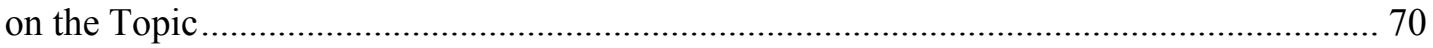

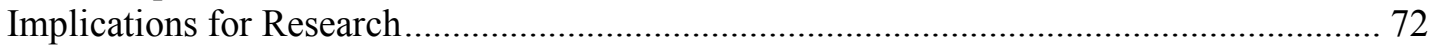

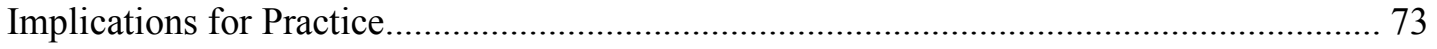

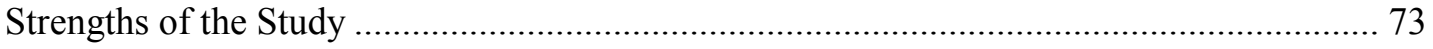

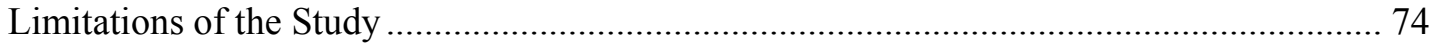

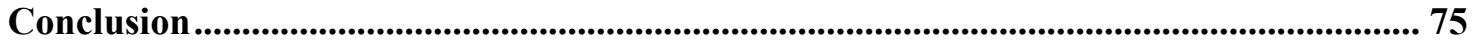

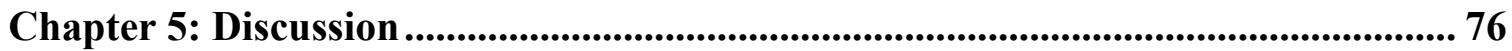

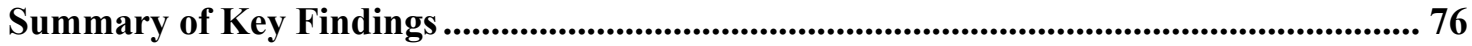

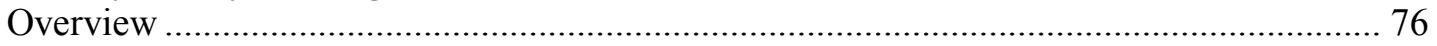

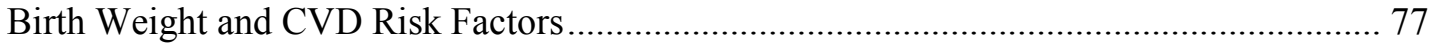

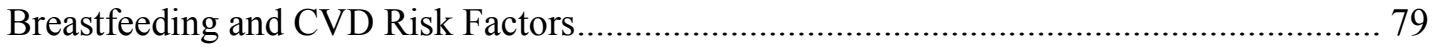

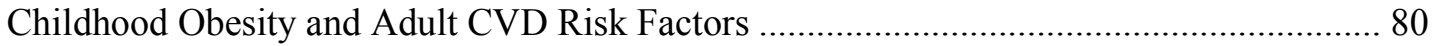

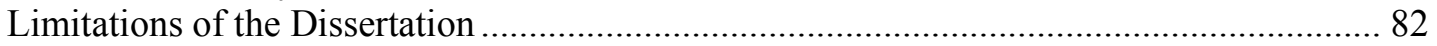

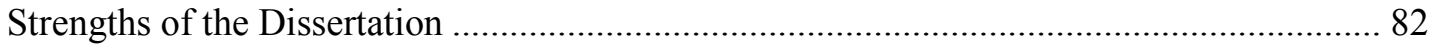

Potential Public Health Implications and Future Recommendations ..................................... 83

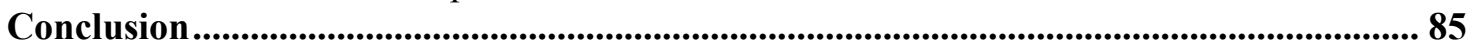

References ................................................................................................................................. 85

Chapter 1 1................................................................................................................................................. 86

Chapter 2 ....................................................................................................................................... 93

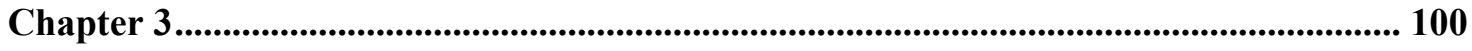

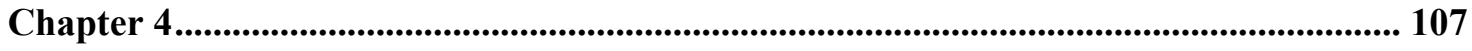

Chapter 5...................................................................................................................................................... 114 


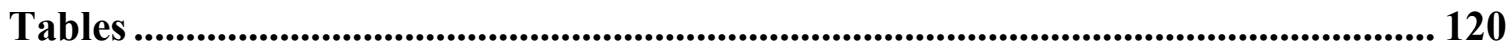

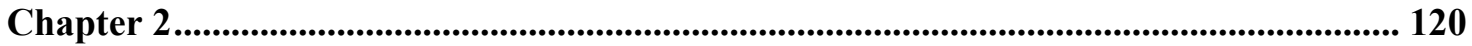

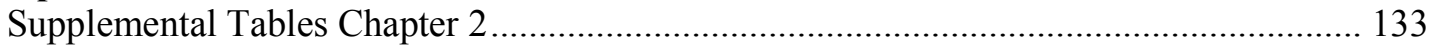

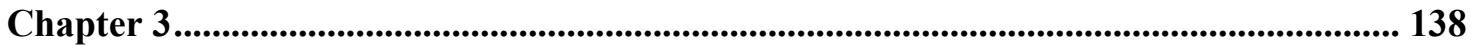

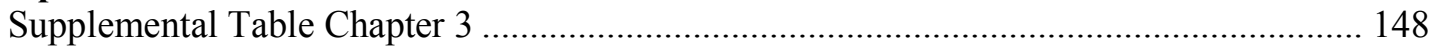

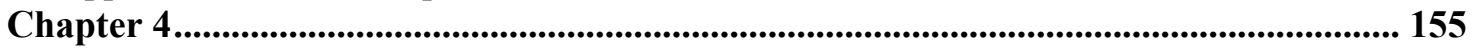

Figures........................................................................................................................................... 163

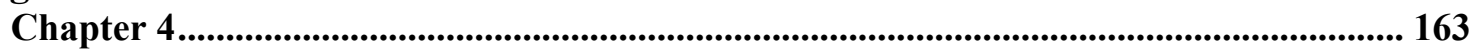

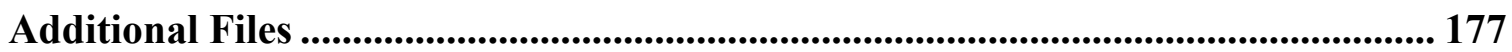

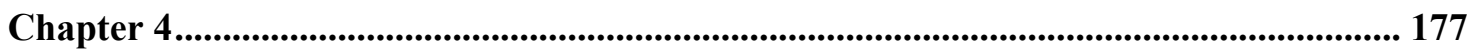




\section{CHAPTER 1: Project overview \\ Introduction}

\section{Cardiovascular Disease (CVD)}

Cardiovascular Disease is the leading cause of death in the world and also in the United States (U.S.). According to the Global Burden of Disease Study, more than 17.3 million people died from CVD in 2013, representing 31\% of all global deaths ${ }^{1,2}$ and nearly $50 \%$ of deaths from chronic diseases worldwide. ${ }^{3}$ The number of CVD mortalities is projected to rise to more than 23.6 million by $2030 .{ }^{4,5}$ Based on U.S. mortality data, CVD accounted for $34.3 \%$ deaths in $2006,{ }^{6}$ $32.8 \%$ deaths in $2008,{ }^{7} 31.9 \%$ deaths in $2010,{ }^{8}$ and $30.8 \%$ of all deaths in $2013 .{ }^{4}$ Although CVD mortality rate is declining, it is still the leading cause of death in the U.S. and accounts for nearly 1 out of 3 deaths. ${ }^{4}$ According to the 2016 Heart Disease and Stroke Statistics update, more than one third of U.S. adults have one or more than one types of CVD. ${ }^{4}$ For 2011-12, the estimated cost of CVD (direct and indirect) was $\$ 316.6$ billion, which is projected to increase to $\$ 918$ billion by $2030 .{ }^{4}$ There are geographic disparities in the CVD prevalence in the U.S. The Appalachian region in the southeastern U.S. has among the highest rates of CVD compared to rest of the nation. ${ }^{9-11}$ West Virginia (WV), a state entirely within the Appalachian region, has one of the highest CVD mortality rates in the U.S. ${ }^{4}$ In 2013, the age adjusted CVD mortality rate in WV was 270.6 per 100,000 , ranking 47 th in the nation (U.S.: 225.2 per 100,000 population). ${ }^{4}$ With such a high prevalence of CVD, it becomes important to assess the risk factors of CVD in order to implement preventative measures. Some of the known risk factors for CVD include high blood pressure (hypertension), abnormal lipid profile, obesity, diabetes mellitus, and family history of heart disease, which are linked to known risky health behaviors such as smoking, physical inactivity, poor diet, and excessive alcohol intake. ${ }^{12-15}$ Previously these risk factors were thought to be prevalent in adulthood only, but are now detected during early childhood and adolescence as well. ${ }^{16,17}$ These risk factors have the propensity to track from childhood into adulthood and thus are considered predictors of adult CVD risk factors. ${ }^{18}$ As current adolescents enter adulthood, the prevalence of CVD is expected also to rise, ${ }^{19}$ as projected that more than $43 \%$ of men and $45 \%$ of women in the U.S. will have some form of CVD and $41.4 \%$ will have hypertension by $2030 .{ }^{4,20}$

\section{CVD Risk Factors}

For this study we focus on three CVD risk factors: (1) blood pressure measured by systolic blood pressure (SBP) and diastolic blood pressure (DBP), (2) lipids including total 
cholesterol (TC), low-density lipoprotein cholesterol (LDL), high-density lipoprotein cholesterol (HDL), non-high-density lipoprotein cholesterol (non-HDL), and triglycerides (TG), and (3) obesity.

High Blood Pressure. High blood pressure can be due to high SBP $\geq 140 \mathrm{mmHg}$ or high DBP $\geq 90 \mathrm{mmHg}$ or both. Hypertension is defined as SBP and DBP $\geq 140 / 90 \mathrm{mmHg}$ for adults' $\geq 20$ years, and $\geq 95^{\text {th }}$ percentile for age and sex for children and adolescents. ${ }^{21}$ High SBP, high DBP, and hypertension are all independent risk factors for CVD. ${ }^{22-24}$ Hypertension is responsible for approximately two-thirds of stroke and one-half of ischemic heart diseases. ${ }^{25,26}$ According to National Health and Nutrition Examination Survey (NHANES) 2009-2012 data, nearly one third (32.6\%) of U.S. adults' $\geq 20$ years have hypertension. ${ }^{27}$ Among children and adolescents ages 8 $17,11.0 \%$ have pre-hypertension (SBP or DBP $\geq 90$ but less than 95 percentile) or hypertension (SBP or DBP $\geq 95$ th percentile), which remained unchanged since 1999-2000.4,27 A systematic review with meta-analysis concluded that high blood pressure tracks strongly from childhood into adulthood with the average tracking correlation coefficients of 0.38 and 0.28 for SBP and DBP respectively. ${ }^{28}$ Not only is hypertension in adulthood one of the leading causes of heart diseases in the U.S. ${ }^{29,30}$, but children with hypertension may also have evidence of cardiovascular endorgan damage such as increased left ventricular mass and thickness of carotid artery intima media (potential markers of hypertensive vascular damage). ${ }^{31-33}$ According to the United States Center for Disease Control and Prevention (CDC)'s 2013 Behavioral Risk Factor Surveillance System (BRFSS), the state-specific prevalence of adult hypertension in the U.S. ranges from $24 \%-41 \%$. WV has the highest prevalence of adult hypertension in the nation (rank 50). ${ }^{34}$ Additionally, results from the WV CARDIAC project 2014-2015 found nearly 23\% fifth grade children in WV to have hypertension (SBP and/or DBP $\geq 95 \%$ percentile). ${ }^{35}$

Abnormal serum lipid and lipoprotein concentrations. Abnormal serum lipid and lipoprotein cholesterol levels (high TC, LDL, TG, non-HDL, and low HDL) are another established risk factor for CVD. ${ }^{14,15,36}$ Adults with high TC have nearly twice the risk of heart disease compared to those with normal cholesterol levels. ${ }^{37}$ High cholesterol in early life has also been associated with preclinical atherosclerosis that contributes to adult atherosclerosis. ${ }^{38-41}$ The criteria for high risk for each component of lipid profile in children and adolescents vary by age and gender but are based on having an individual lipid component $\geq 95^{\text {th }}$ percentile. ${ }^{42}$ For $10-19$ year old children the individual components $\geq 95^{\text {th }}$ percentile corresponds to approximately: TC $\geq 200 \mathrm{mg} / \mathrm{dL}, \mathrm{LDL} \geq 130 \mathrm{mg} / \mathrm{dL}, \mathrm{TG} \geq 130 \mathrm{mg} / \mathrm{dL}$ HDL $<40 \mathrm{mg} / \mathrm{dL}$, and non-HDL $\geq 145 \mathrm{mg} / \mathrm{dL}$. ${ }^{42-}$ ${ }^{44}$ For adults the criteria for high risk for each component of lipid profile is: TC $\geq 240 \mathrm{mg} / \mathrm{dL}$, $\mathrm{LDL} \geq 160 \mathrm{mg} / \mathrm{dL}, \mathrm{TG} \geq 200 \mathrm{mg} / \mathrm{dL}$, and HDL $<40 \mathrm{mg} / \mathrm{dL} .{ }^{45,46}$ According to the 2016 Heart 
Disease and Stroke Statistics update, ${ }^{4}$ mainly based on NHANES 2009-2012 data showed that the prevalence of high TC was $13 \%$ in U.S. adults $\geq 20$ years and $8.5 \%$ in adolescents ages 12-19.

High LDL was prevalent in $27 \%$ of U.S. adults' $\geq 20$ years and nearly $7 \%$ in adolescents ages 12 19. The proportion of low HDL varied significantly by gender. Nearly $25 \%$ of men and less than $10 \%$ of women $\geq 20$ years had low $\mathrm{HDL}^{47}$ and $19.5 \%$ of boys and $11 \%$ of girls (ages $12-19$ ) had low HDL. ${ }^{4}$ For TG nearly a quarter $(25.1 \%)$ of adults' $\geq 20$ years had high TG and the proportion in children and adolescents $12-19$ years old, $10 \%$ males and $6.5 \%$ females had high TG. ${ }^{4}$ Based on CDC's BRFSS 2013, WV ranks 48th in the nation with the prevalence of high TC among adults. ${ }^{34}$ Additionally, results from the WV CARDIAC project 2014-2015 showed that nearly 5\% of children in fifth grade have high LDL $(\geq 130 \mathrm{mg} / \mathrm{dL})$ and $17 \%$ have low HDL $(<40 \mathrm{md} / \mathrm{dL}){ }^{35}$ Although nationally the prevalence of high total cholesterol and low HDL is declining in children ages $8-17 ;{ }^{27}$ it is still an important CVD risk factor. Furthermore, as observed with high blood pressure, abnormal serum lipid and lipoprotein also track from childhood into adulthood as well. ${ }^{48-51}$

Overweight and obesity. Overweight and obesity is another major risk factor for CVD. ${ }^{52}$ Body Mass Index (BMI) is a person's weight in kilograms divided by the square of height in meters used to establish the weight status of an individual. However, at a population level it is one of the most commonly used measures to assess body fatness. BMI correlates well with the individual's adiposity status, and is also a convenient and an inexpensive method. ${ }^{53,54}$ Overweight is defined as $25.0 \leq \mathrm{BMI} \leq 29.9 \mathrm{~kg} / \mathrm{m}^{2}$, and obese is defined as BMI $\geq 30.0 \mathrm{~kg} / \mathrm{m}^{2}$ in adults. ${ }^{55} \mathrm{In}$ children overweight is defined as BMI ranging from 85 th to $<95$ th percentile, and obesity is defined as $\geq 95$ th percentile using sex-specific BMI-for-age 2000 CDC growth charts. ${ }^{56}$ Overweight and obesity during childhood and adolescence is a major public health problem. According to the most recent national study (NHANES 2011-2012), nearly 32\% children and adolescents between the ages of 2 to 19 were either overweight or obese, of which $17 \%$ were obese. The study stratified the results by different age groups and found that nearly $8 \%$ of 2-5year-olds were obese compared to $18 \%$ of $6-11$-year-olds and $21 \%$ of $12-19$-year-olds. ${ }^{57}$ Data from the WV Healthy Lifestyles Act Evaluations 2008-2009 shows that 18.5\% of children in kindergarten were obese, compared with $22.1 \%$ of second graders and $29.6 \%$ of fifth graders. ${ }^{58}$ According to the 2007 National Survey of Children's Health (NSCH) nearly 36\% of WV adolescents ages 10-17 years were overweight or obese compared to the national average of $32 \% .{ }^{59}$ Results from the WV CARDIAC project $2014-2015$ showed that nearly $47 \%$ of children in fifth grade were either overweight or obese (BMI $\geq 85$ th percentile). ${ }^{35}$ 
Overweight adolescents have a 40\%-80\% chance of becoming overweight or obese adults $^{60,61}$ and adult adiposity is a well-known risk factor for developing adult CVD. ${ }^{62-64}$ Some of the immediate heath implications of pediatric obesity include development of other CVD risk factors during childhood and adolescence ${ }^{65-70}$ Adult CVD risk factors found during childhood (such as high blood pressure, poor lipid profile, impaired glucose tolerance, and metabolic syndrome ${ }^{71-73}$ ) are amplified in the presence of pediatric obesity and referred by Ford and colleagues as 'obesity-associated CVD risk factors'. ${ }^{71}$ A population-based study estimated that $70 \%$ of obese children and adolescents between the ages of 5 to 17 have at least one risk factor for CVD. ${ }^{65}$ Data from the longitudinal Bogalusa Heart Study shows that overweight children were 4.5 and 2.4 times as likely to have elevated SBP and DBP, compared to normal weight children respectively. ${ }^{74}$ Moreover, overweight children were 2.4 times as likely to have high TC, 3 times likely to have high LDL, 3.4 times as likely to have low HDL, and 7 times as likely to have high TG compared to normal weight children. ${ }^{74} \mathrm{~A}$ recent systematic review with metaanalysis concluded that obese children, when compared to normal weight children, have raised SBP by $7.49 \mathrm{mmHg}$, DBP by $4.45 \mathrm{mmHg}$, and TC by $0.15 \mathrm{mmol} / \mathrm{L} .^{70}$

Moreover, data from NHANES 2011-12 showed that two-third of adults are either overweight or obese $(68.5 \%)$ in the U.S. The prevalence of extreme obesity $(6.3 \%$; BMI $\geq 40.0$ $\left.\mathrm{kg} / \mathrm{m}^{2}\right)$ was higher in women $(8.3 \%$; $95 \%$ CI: $6.9,9.8)$ compared to men $(4.4 \%$; $95 \%$ CI: 2.8 , 6.8) ${ }^{57}$ According to the 2014 BRFSS data, the state specific obesity rates ranged from $21.3 \%$ percent in Colorado to $35.9 \%$ in Arkansas. In the past decade the obesity rates in WV has increased from $27.6 \%$ in 2004 to $35.7 \%$ in 2014 , ranking the second highest rate of adult obesity in the nation. ${ }^{75}$

Adult obesity is also associated with numerous comorbid conditions such as hypertension, dyslipidemia, diabetes, insulin resistance, and metabolic syndrome and all these conditions increases the risk for CVD. ${ }^{76}$ Obese individuals have an increased risk of developing hypertension. Some studies have suggested that $60-75 \%$ of hypertension can be attributed to adult overweight or obesity. ${ }^{62,77,78}$ Furthermore, obesity is a predictor of higher TG, LDL, and lower HDL. ${ }^{62}$ Results from a meta-analysis of 21 cohort studies concluded that overweight was an independent risk factor for heart diseases, but the association was partially mediated by the effects of overweight on blood pressure and cholesterol levels, which accounted for nearly $45 \%$ of the increased risk of heart disease. ${ }^{79}$ Data from NHANES III showed a strong association of adult body mass index (BMI) with hypertension and abnormal lipid profile. The report showed that the prevalence of high blood pressure was $15 \%$ for underweight/normal weight men and women, and $38 \%$ for obese women and $42 \%$ for obese men. ${ }^{80}$ 
Link between childhood obesity and adult CVD risk factors. In summary, research suggests that childhood obesity is an independent risk factor for developing childhood CVD risk factors, ${ }^{65-68}$ and obese children also have higher risk of becoming overweight/obese adults, ${ }^{60,61,81}$ which is an independent risk factor for developing adult CVD risk factors. ${ }^{62,64,76-78}$ However, studies that have examined the direct relationship between childhood obesity and adult CVD have yielded inconsistent results. ${ }^{63,71,82-84}$ Recent systematic reviews suggest that the relationship between childhood obesity and adult high blood pressure or poor lipid profile is weak, possibly because the results are confounded by adult obesity. ${ }^{84,85}$ Thus, it remains unclear whether childhood obesity is an independent risk factor for adult CVD risk factors or whether childhood obesity persists as adult obesity and indirectly increases the risk of adult CVD. ${ }^{86,87}$

\section{Early Life Exposure and Origins of Childhood and Maternal CVD Risk Factors}

There is growing evidence that early-life risk factors may influence the development of fatty streaks and vascular endothelial dysfunction in children to atherosclerotic plaques in early adulthood and subsequent CVD in later life.$^{88-91}$ For example, research shows an association between birth weight and obesity, ${ }^{92}$ hypertension, ${ }^{93}$ and dyslipidemia. ${ }^{94}$ Conversely, early-life protective factors (such as breastfeeding) have been shown to reduce the risk of overweight and obesity, ${ }^{95}$ hypertension ${ }^{96,97}$ and improve cholesterol concentrations in later life. ${ }^{98}$

These relationships may start to occur prior to birth. The Barker hypothesis (also called the 'in utero fetal programming hypothesis' or the 'developmental origins of adult disease hypothesis') proposed that intrauterine malnutrition leads to low birth weight (LBW) infants, which predisposes infants to develop CVD in later life. ${ }^{99-102}$ Although this hypothesis has been a topic of great controversy, evidence seems to be accumulating on various associations between perinatal risk factors and the development of CVD risk factors in subsequent years. ${ }^{103}$ Although, Barker and colleagues focused primarily on LBW and adult CVD, recent literature has focused on other perinatal risk factors such as high birth weight (HBW), maternal pre-pregnancy adiposity, excessive gestational weight gain (GWG), and gestational diabetes as early determinants for the origins of childhood and adulthood CVD..$^{92,94,104-108}$ Some studies have shown that it is not only in the periods of gestation but also in the critical period of lactation (e.g., breastfeeding) that the type of nutrition can have long lasting effects on the offspring's development of CVD risk factors in later life. ${ }^{109,110}$

Surprisingly, perinatal risk factors not only impact the health of the offspring, but also are associated with maternal CVD morbidity and mortality in later years. ${ }^{111-115}$ Data shows that mothers who deliver LBW infants have significantly higher risk of maternal CVD morbidity and 
mortality ${ }^{116-120}$ and CVD related risk factors such as hypertension ${ }^{121}$ and metabolic syndrome. ${ }^{122}$ Furthermore, recent literature indicates that mothers who breastfeed their newborns have a significant positive impact on their own cardiovascular health ${ }^{115}$ including lower prevalence of CVD risk factors (e.g., high blood pressure and poor lipid profile). ${ }^{123}$

\section{Distal Determinants of CVD Risk Factors}

There is an ongoing debate on using the term 'distal' and 'proximal' causes of disease/outcome in the field of public health research. Social and behavioral sciences, a branch of public health research that focuses on social determinants of health use the term 'distal' for social and economic risk factors and 'proximal' for individual and biological factors. ${ }^{124,125}$ Others suggest that proximal are more direct or 'downstream' factors and distal are indirect or 'upstream' health determinant for the outcome of interest. ${ }^{124}$ For the purpose of this current dissertation, distal determinant of CVD risk factors are predictor variables, which are temporally distal (far in relation to time) from when the health outcomes are being assessed. We focused on two distal perinatal factors (birth weight of the infant and breastfeeding) and later childhood and maternal CVD risk factors for study 1 and study 2 (aims 1 and 2 respectively). For the third study we focused on childhood obesity as the distal determinant of adult CVD risk factors.

\section{Summary}

CVD morbidity and mortality accounts for nearly one-third of all diseases and death nationally and worldwide. Although the mortality from CVD is declining in the U.S. since the past decade, CVD morbidity is expected to increase from nearly $33 \%$ to more than $43 \%$ by 2030. ${ }^{4,20}$ Several well-established adult CVD risk factors (i.e. high blood pressure, high cholesterol, and obesity) are now prevalent during childhood as well. Childhood obesity in itself is an independent risk factor for other childhood CVD risk factors including high blood pressure and abnormal lipid profile.

Additionally, the development of CVD risk factors may start as early as in utero and infancy, and these early life exposures can impact the cardiovascular health in later years. CVD risk factors such as childhood obesity, high blood pressure, and dyslipidemia share common early life exposures such as birth weight and breastfeeding. However, there is limited data focusing on these associations during childhood independent of child's obesity status. Surprisingly, these perinatal risk factors (birth weight and breastfeeding) have been linked to maternal CVD risk factors in subsequent years. ${ }^{120,122,126-128}$ However, this topic is a recent and an active area of 
research with limited number of studies have explored these associations for maternal cardiovascular health in subsequent years. Most relevant to our study, no studies examined these associations longitudinally across the lifespan in the state of WV; where there is a vital need to assess these risk factors and implement early preventative measures to reduce the high burden of CVD in this state. Data shows that WV has some of the highest rates of obesity, hypertension, high cholesterol levels, LBW infants, and one of the lowest breastfeeding rates in the nation. Thus, we intended to fill this gap in the literature with the first two studies/aims.

For the third aim we moved our focus from perinatal exposures (birth weigh and breastfeeding) and its associations with childhood and maternal CVD risk factors to childhood exposure (over weight and obesity) and its association with adult CVD risk factors. Despite the high prevalence of both childhood obesity and CVD, studies examining the relationship between childhood adiposity and adult CVD have yielded conflicting results. ${ }^{63,71,82-84}$ However, adult adiposity is an established risk factor for developing adult $\mathrm{CVD}^{62,76-78}$ and there is much evidence to suggest that overweight children and adolescents have a higher risk of becoming overweight or obese adults. ${ }^{60,61,81}$ Thus, it remains unclear whether childhood obesity is an independent risk factor for adult CVD risk factors or whether the association is mediated through adult obesity. ${ }^{84-87}$ Given that childhood obesity as an independent risk factor for CVD in adults is not well established, for the third aim/study we examined the association between childhood obesity and adult CVD risk factors.

The overall objective of this project was to determine the associations between two distal perinatal factors (birth weight and breastfeeding) and childhood and maternal cardiovascular health, and determine the relationship between childhood obesity as the distal determinant of adult CVD risk factors. The central hypothesis of this project is that early life exposures are independently associated with childhood CVD risk factors and childhood obesity is an independent risk factor for adult CVD risk factors. Specifically, the proposed project looks to investigate the following specific aims.

\section{Specific Aim 1}

Investigate the association between infant's birth weight on childhood CVD risk factors (SBP, DBP, TC, LDL, HDL, non-HDL, and TG) at 11 years of age (controlling for child's current body mass index (BMI), and the subsequent risk of developing maternal CVD risk factors (TC, LDL, HDL, non-HDL, and TG) 11 years post partum. 
Objective 1.1. Determine the association between infant's birth-weight on childhood CVD risk factors (SBP, DBP, TC, LDL, HDL, non-HDL, and TG) at 11 years of age independent of childhood adiposity.

Working Hypotheses 1.1. Low or/and high birth weight infants will have significantly higher SBP, DBP, TC, LDL, HDL, and TG, and lower HDL at 11 years of age independent of childhood adiposity.

Objective 1.2. Determine the association between infant's birth-weight on maternal CVD risk factors (TC, LDL, HDL, non-HDL, and TG) 11 years post-partum.

Working Hypothesis 1.2. Mothers who gave birth to low or/and high birth weight infants will have significantly higher TC, LDL, non-HDL, and TG, and lower HDL, 11 years post-partum.

\section{Specific Aim 2}

Investigate the association between infant breastfeeding and childhood CVD risk factors (SBP, DBP, TC, LDL, HDL, non-HDL, and TG) at 11 years of age (controlling for child's current BMI, and the subsequent risk of developing maternal CVD risk factors (TC, LDL, HDL, non-HDL, and TG) 11 years post partum.

Objective 2.1. Determine the association between infant breastfeeding and childhood CVD risk factors (SBP, DBP, TC, LDL, HDL, non-HDL, and TG) at 11 years of age independent of childhood adiposity.

Working Hypotheses 2.1. Infants who were breastfed have significantly lower SBP, DBP, TC, LDL, non-HDL, and TG and higher HDL at 11 years of age independent of childhood adiposity.

Objective 2.2. Determine the association between infant breastfeeding and maternal CVD risk factors (TC, LDL, HDL, non-HDL, and TG) 11 years post-partum.

Working Hypothesis 2.2. Mothers who breastfed their infants will have significantly lower TC, LDL, non-HDL, and TG and higher HDL after 11 years post-partum.

\section{Specific Aim 3}

Conduct a comprehensive systematic review (SR) and meta-analysis (MA) to critically evaluate the available evidence regarding the relationship between childhood obesity and adult CVD risk factors (SBP, DBP, TC, LDL, HDL, non-HDL, and TG).

Objective 3.1. Determine the overall effect of childhood and adolescence obesity and its association with adult blood pressure and lipid profile. 
Objective 3.2. Identify sources of between-study heterogeneity in relation to changes in adult blood pressure and lipid profile as a result of childhood and adolescence obesity. Where significant between study variation exists in research methodology, study design, study population, sample size, analysis and context, we will determine covariates responsible for such heterogeneity for the primary outcome variables (SBP, DBP, TC, LDL, HDL, non-HDL, and TG) of interest.

The three specific aims/studies correspond to chapters two, three, and four. Chapter 5 presents a summary of key findings of individual studies, strengths, limitations, and significance of the studies, potential public health implications as well as potential direction and recommendations for future research. There are wide-ranging factors that contribute to the high burden of CVD across the life course. Thus examining the associations of these distal determinants of CVD will aid in the understanding of these factors and their potential role. This can potentially lead to planning and implementation of ideal preventive strategies for promoting cardiovascular health during various life stages. 


\title{
CHAPTER 2: Birth weight and childhood and maternal cardiovascular disease risk factors
}

\begin{abstract}
Introduction: The reported associations between birth weight and childhood cardiovascular disease (CVD) risk factors have been inconsistent. The relationship between infants' birth weight and later maternal CVD is also a more recent and active area of research. We aimed to examine the association between birth weight and subsequent childhood and maternal CVD risk factors 11 years post-partum.

Methods: The study used longitudinally linked data from three cross-sectional datasets [West Virginia Birth Certificates, the Working in Appalachia to Track High Birth Score, Critical Congenital Heart Disease and Hearing Loss (WATCH)/Birth Score project, and the Coronary Artery Risk Detection in Appalachian Communities (CARDIAC) project] in West Virginia and restricted to full term infants only. The outcome variables included blood pressure measures (systolic blood pressure (SBP), diastolic blood pressure (DBP)) for children and lipid levels for both mothers and children (total cholesterol (TC), low-density lipoprotein cholesterol (LDL), high-density lipoprotein cholesterol (HDL), non-high-density lipoprotein cholesterol (non-HDL), and triglycerides (TG). The exposure was birth weight of the infant assessed as continuous and categorical variable using conventional cut-offs. Low birth weight (LBW) and high birth weight (HBW) was defined as birth weight of $<2,500$ grams and $>4000$ grams respectively. The role of child's current body mass index (BMI) was assessed as a potential mediator.

Results: The final study sample after excluding infants born pre-term (i.e., $<37$ weeks of gestation: $\mathrm{N}=2,097,9.67 \%$ ) was 19,583 . In this sample, nearly $3 \%$ of the infants were LBW and $11 \%$ were HBW. Unadjusted analyses showed a positive association between birth weight and the SBP, DBP, HDL, and a negative association between birth weight and TGs. When adjusted for the child's BMI, the association became negative but non-significant for SBP, and remained positive but became non-significant for DBP. The association between birth weigh and HDL $[b=$ $0.14 \mathrm{mg} / \mathrm{dL}(95 \% \mathrm{CI}: 0.11,0.18)$ per $1000 \mathrm{~g}$ increase in birth weight] and between birth weight and $\log$-TG $[b=-0.007(-0.008,-0.005)$ per $1000 \mathrm{~g}$ increase in birth weight] remained significant after adjustments were made for the child's BMI. LDL and non-HDL became significant and negatively associated with birth weight in the adjusted analysis [LDL $(b=-0.1 \mathrm{mg} / \mathrm{dL}(-0.19$, $0.16)$ per $1000 \mathrm{~g}$ increase in birth weight; non-HDL $(\mathrm{b}=-0.18 \mathrm{mg} / \mathrm{dL}(-0.28,-0.09)$ per $1000 \mathrm{~g}$ increase in birth weight]. There was a positive association between birth weight and maternal TC levels, which became non-significant in the adjusted analysis $[b=0.4 \mathrm{mg} / \mathrm{dL}(95 \% \mathrm{CI}:-0.01$, 0.90) per $1000 \mathrm{~g}$ increase in birth weight]. None of the other maternal lipids levels (LDL, HDL, non-HDL, and TG) were significant in the unadjusted or the adjusted analysis.

Conclusion: In this young Appalachian population, birth weight was associated with lipid levels in children. LBW was associated with higher LDL, non-HDL, and TGs and lower HDL levels in fifth grade children independent of the current weight status. As CVD risk factors persist from childhood into adulthood, these small effect sizes can have potential unfavorable consequence on lipid levels in later adulthood. HBW seemed to show a trend towards poor maternal lipid levels 11 years post-partum. Well-designed longitudinal studies are needed to understand the complex pathways examining these associations at different life stages.
\end{abstract}




\section{Introduction}

Cardiovascular Disease (CVD) is the leading cause of death globally, nationally, and also in the state of West Virginia (WV). ${ }^{1-3} \mathrm{WV}$, a state entirely within the Appalachian region, has one of the highest CVD mortality rates in the U.S. ${ }^{3}$ In 2013, the age adjusted CVD mortality rate in the United States was 225.2 per 100,000 population whereas in WV the age adjusted CVD mortality rate was 270.6 per 100,000 , ranking 47 th in the nation. ${ }^{3}$ High blood pressure and poor cholesterol are well-established risk factors for CVD. ${ }^{4-8}$ These risk factors are not only found in adulthood but are prevalent during childhood as well and have been linked with numerous perinatal exposures such as birth weight. ${ }^{9-17}$

The fetal origins hypothesis proposed by Barker and colleagues states that early life exposures such as under nutrition in utero could potentially increase susceptibility to poor health outcomes in later life. ${ }^{18}$ Briefly, the hypothesis suggests that this poor nutrition in utero can potentially trigger a response where the fetus may slow down its own growth to adapt to the unfavorable conditions (restricted nutrients and poor fetal oxygenation). This potentially leads to inadequate fetal growth (intrauterine growth restriction (IUGR)),${ }^{19}$ as a short-term strategy for survival. This strategy leads to having low birth weight (LBW) but in the long term can have adverse health consequences. ${ }^{20}$

LBW is defined as birth weight of $<2,500$ grams (5.5 lbs) and high birth weight (HBW) is defined as a birth weight of $>4000$ grams $(8.8 \mathrm{lbs}) .{ }^{21-23} \mathrm{LBW}(<2,500$ grams $)$ at birth can result from either IUGR in a full-term infant ( $\geq 37$ weeks of gestation), or from premature/preterm birth ( $<37$ weeks of gestation). ${ }^{19}$ According to National Vital Statistics System the national average incidence of LBW is $8.0 \%$ of all live births, based on 2013 birth certificates data. The statespecific incidences vary from 5.8\% in Alaska to $11.5 \%$ in Mississippi. WV ranks 45 in the nation, with an incidence of 9.4\%. ${ }^{24}$ Based on 2011 Pregnancy Surveillance System (PNSS) and Pediatric Nutrition Surveillance System (PedNSS) the national prevalence of LBW and HBW is $8.9 \%$ and $6.4 \%$ respectively in low-income children enrolled in federally funded programs. ${ }^{23}$

A child's LBW is an indicator of suboptimal fetal growth and an important predictor for several long-term health outcomes including morbidity and mortality from CVD. ${ }^{25-27}$ However, others have argued that birth weight is a strong predictor of infant mortality in the first year of life and not a predictor for the development of chronic diseases during childhood or adulthood. ${ }^{28,29}$ Finally, recent literature also shows that mothers who deliver LBW or HBW infants have a higher risk of having CVD morbidity and mortality. ${ }^{30,31,58}$ 
The detailed literature review presented below first outlines associations between birth weight and child CVD risk factors including blood pressure (systolic blood pressure (SBP), diastolic blood pressure (DBP)), and lipid profile ((total cholesterol (TC), low-density lipoprotein cholesterol (LDL), high-density lipoprotein cholesterol (HDL), non-high-density lipoprotein cholesterol (non-HDL), and triglycerides (TG)), and then expands on associations between birth weight and maternal CVD risk factors.

\section{Child Birth Weight and Child CVD Risk Factors}

LBW has shown to be an independent risk factor for childhood CVD risk factors such as obesity ${ }^{32}$ high blood pressure, ${ }^{33,34}$ and poor lipid profile. ${ }^{35}$ For example, Gademan and colleagues found that birth weight was significant and inversely associated with SBP and DBP independent of the child's current body mass index (BMI) at 6 years of age. ${ }^{33}$ However, Zhang and colleagues observed the inverse association only with SBP but a positive (linear) association with DBP. The study demonstrated that among children 6-15 years, SBP levels decreased $0.9 \mathrm{mmHg}(\mathrm{b}=-0.9 ; \mathrm{p}$ $=0.002)$ and DBP increased $0.9 \mathrm{mmHg}(\mathrm{b}=0.9 ; \mathrm{p}=0.006)$ for each $1,000 \mathrm{~g}$ increase in birth weight. ${ }^{36}$ Other studies also found a positive (linear) relationship between birth weight and high blood pressure. ${ }^{9,37,38}$ Filler and colleagues observed that birth weight was significantly and positively correlation with DBP $z$ score $(r=0.037, p=0.044)$, BMI z score $(r=0.123, p<0.0001)$ but not with SBP $(r=-0.006, p=0.729) .{ }^{37}$ Sousa and colleagues found that HBW adolescents $(14$ years old) had a significantly higher prevalence ratio for high SBP of 3.3 (95\% CI: 1.7, 6.4) compared with normal birth weight adolescents. ${ }^{38}$ As suggested by these conflicting relationships between blood pressure and birth weight, another study that examined the association at 7 years and 16 years ( 2 different cohorts) found a non-linear relationship and concluded that both LBW and HBW were associated with high SBP. ${ }^{39}$ A recent systematic review concluded that children and adults who were born preterm or very LBW $(<1500 \mathrm{~g})$ have moderately higher SBP later in life, ${ }^{26}$ and a recent meta-analysis also demonstrated an inverse linear association between birth weight and later risk of high SBP. ${ }^{40}$ However, other studies have found a weak association or no association between birth weight and subsequent risk of developing high blood pressure. ${ }^{41-43}$ This also includes a recent systematic review that found no significant association between LBW and childhood obesity or childhood hypertension. ${ }^{44}$ This relationship may be further obscured by the child's current weight status. For example, Berge and colleagues found an association between LBW and SBP only among overweight children between the ages of 5-9 years..$^{45}$

These conflicting relationships are also found when examining the association between birth weight and cholesterol levels in childhood. For example, when examining the association 
between birth weight and serum lipid profile, Donker and colleagues found an inverse association between birth weight and elevated TG concentrations in later childhood (ages 9-11 years). ${ }^{35}$ Another longitudinal study found that among children (ages 4-11 years) LBW was associated with lower mean HDL, higher LDL, and TG levels irrespective of age or gender. ${ }^{34}$ However, Azadbakht and colleagues demonstrated that both LBW and HBW were significantly associated with low levels of HDL. ${ }^{9}$ Similarly, a recent study found no significant association between birth weight (when analyzed as a continuous variable) and lipid profiles, but when birth weight was categorized as low, normal, and high, the results showed that HBW subjects had higher TC levels $(b=0.971 ; p<0.001)$ and higher non-HDL levels $(b=0.960 ; p=0.001)$ compared to children who had normal birth weight. ${ }^{36}$ A study using WV Coronary Artery Risk Detection In Appalachian Communities (CARDIAC) data on 11 years old children found that infants who were born preterm had higher levels of TGs compared to term infants. The study also categorized gestational age categories, as small for gestational age (SGA), appropriate for gestational age (AGA), and large for gestational age (LGA) based on Fenton's weight graphs, ${ }^{46}$ and found that SGA infants were less likely to be obese in fifth grade, but those who were obese had higher TG levels compared to AGA or LGA obese children. ${ }^{47}$ Other studies have found no significant association between HBW and serum lipid profile in children (6 years of age) or between LBW and serum cholesterol levels at 8 years of age, ${ }^{10,48}$ in adolescents, ${ }^{38}$ or in early adulthood. ${ }^{49}$ Consistent with these conflicting results, a recently published systematic review and metaanalysis concluded that the current birth weight standards are poor predictors for later development of adverse health outcomes. ${ }^{44}$

\section{Child Birth Weight and Maternal CVD Risk Factors}

Mothers who deliver LBW infants (BTW $<2500 \mathrm{~g}$ vs. $>3500 \mathrm{~g}$ ) have 7-11 times the risk of maternal CVD mortality in later years. ${ }^{30,50-53}$ Preterm delivery and delivery of a SGA infant has also been associated with an increase risk of maternal CVD. ${ }^{54-56}$ Catov and colleagues found that women who gave birth to preterm babies (independent of hypertension during pregnancy) had higher blood pressure several years after pregnancy compared to women who gave birth to term babies. ${ }^{57}$ Results from a large retrospective cohort study $(\mathrm{N}=129,290)$ found that mothers who delivered babies in the lowest birth weight quintile for gestational age had higher risks of maternal CVD morbidity 15-19 years later compared to mothers who delivered babies in the highest four quintiles grouped together. ${ }^{53}$ One study found that mothers of infants with HBW were more likely to be obese and have higher rates of metabolic syndrome 8 years after delivery. ${ }^{58}$ The study found no significant relationship between the infant birth weight and 
maternal TC, LDL and HDL but found significant positive association for TGs only (Odds Ratio $=2.17 ; 95 \% \mathrm{CI}: 1.01,4.70) .{ }^{58}$ Thus, suggesting that HBW was associated with higher TGs. Another study demonstrated a positive association between delivering a LBW infant and maternal hypertension in later life. ${ }^{59}$ Yet another study that examined the relationship between offspring birth weight and parental carotid artery intima media thickness found that the relationship was significant and inversely associated. ${ }^{60}$ The researchers state two plausible explanations: 1) Maternal environmental factors (e.g., poor diet) can potentially increase maternal risk of atherosclerosis and impact the infant's birth weight or 2) a potential common genetic factor could be related to both LBW and higher risk of atherosclerosis in subsequent years. ${ }^{60}$

\section{Low Birth Weight, Preterm Births, and Small for Gestational Age}

Birth weight of the infant is the weight of the infant at the time of delivery. Preterm birth is defined as birth at less than 37 weeks of gestational age, which can be due to several factors such as infection and/or fetal growth restriction. SGA is defined as an infant who is less than the 10th percentile of birth weight for gestational age. SGA is most often used as a marker of intra uterine growth restriction (IUGR). A LBW may be due to preterm birth or due to IUGR. ${ }^{19}$ Although there seems to be an overlap between these terms, Behrman and colleagues suggest that "They are not interchangeable, however, as each has distinct etiologies and risk factors. Among low birth weight infants, approximately two-thirds are born preterm, whereas less than 20 percent of SGA age infants are born preterm." ${ }^{61} \mathrm{LBW}$ and HBW babies are found in both preterm and full term babies. However, preterm infants are at a greater risk of infant mortality as well as adverse health outcomes compared to term birth infants. ${ }^{61-64}$ Many researchers believe that birth weight categories (LBW, normal birth weight, HBW) and birth weight for gestational age categories (SGA, AGA, and LGA), both combines preterm and term births and are thus unreliable predictor of population risk. They further suggest that these two populations should be examined independently; for example, Wilcox notes, "Once the percent of preterm births is known, the analysis of birth weight can be simplified by restricting the sample to term births. Among term births, the influence of gestational age is minor and can be ignored." ${ }^{28}$ Lastly, some of the critics of Barker hypothesis have argued that Barker and colleagues failed to account for infants born prematurely. ${ }^{65}$ They argue that not only the determinants of preterm birth and fetal growth differ; ${ }^{66}$ IUGR, rather than prematurity is associated with later chronic diseases. ${ }^{67}$ 


\section{Statement of the Problem}

As presented in the literature review on the current topic above, most studies found an inverse association between birth weight and CVD risk factors without excluding preterm infants or only focusing on preterm infants as demonstrated by earlier systematic reviews. ${ }^{26,68}$ Our study population included nearly $10 \%$ preterm birth infants. Furthermore, the LBW in the overall population was $7 \%$, which accounts for 3\% among full-term infants, and nearly $50 \%$ in preterm infants (49.36\%). Thus, in agreement with the literature that suggests that preterm and term babies are two distinct populations, we aimed to examine the association of birth weight and CVD risk factors in full-term birth infants only. The association of birth weight in full-term births with CVD risk factors is less clear and our study is intended to fill this gap.

Moreover, we can also observe from the literature that the associations between birth weight and childhood CVD risk factors show inconsistent results. While some studies have found a significant association ${ }^{33-35}$ others have found a weak association or no association between birth weight and poor lipid profile or blood pressure in subsequent years. ${ }^{41-43,69,70}$ Child's birth weight has also shown to be associated either linearly (negatively or positively) or non-linearly (Ushaped) with childhood obesity, ${ }^{32,38,71-74}$ which is an independent risk factor for childhood CVD risk factors. Thus many argue that child's current weight may be a potential mediator between these associations, while others argue that socio-demographic factors and maternal history of CVD may increase the risk of LBW, as well as increase the risk of CVD risk factors in the offspring. ${ }^{75,76}$ Thus this study aims to examine this association adjusting for important confounder variables as well as examine the role of childhood BMI as a potential mediator. There is also a paucity of information on the influence of infant's birth weight and the subsequent risk of maternal hypertension and only one study in India looked at the association for maternal lipid profile postpartum. ${ }^{58}$ Most importantly, we did not find any study examining these associations (for both children and mothers) longitudinally in the state of WV; where there is a critical need to identify risk factors that can potentially reduce the high burden of CVD through early prevention and intervention. For the purpose of this study we focused on two CVD risk factors: (1) blood pressure (SBP and DBP) and, (2) and lipids and lipoprotein levels (TC, LDL, HDL, non-HDL, and TG). The main objective of the study was to determine the association between infant's birth weight and childhood CVD risk factors (SBP, DBP, TC, LDL, HDL, non HDL, and TG) at 11 years of age controlling for child's current BMI, and the subsequent risk of developing maternal CVD risk factors (TC, LDL, HDL, non-HDL, and TG) 11 years post partum. 


\section{Methods and Materials}

\section{Data Sources}

The study used data from three projects, including WV Birth Certificates, the Working in Appalachia to Track High Birth Score, Critical Congenital Heart Disease and Hearing Loss (WATCH)/Birth Score project, and the Coronary Artery Risk Detection in Appalachian Communities (CARDIAC) project. The WV Birth-Score Project began in 1985 and is an infant risk-screening tool. ${ }^{47,77}$ Health care professionals collect data on every newborn within 24-48 hours or prior to discharge, in order to identify infants who are at a high risk of morbidity or mortality in the first year of life, for referral to primary care management. ${ }^{47,77,78}$ In this study, children participating in the Birth-Score project (all of whom are merged with the Birth Certificate data) born between in 1994 to 2000 were merged with data collected by the CARDIAC Project in years 2004-2010. The CARDIAC project collects data on fifth grade public school children in 55 counties in WV with informed consent by parents/guardians and assent by the child. ${ }^{79}$ Area coordinators employed by the project, along with health science student volunteers, local school nurses, and volunteer phlebotomists conduct blood pressure, anthropometric measurements, and blood lipid testing. The blood sample is obtained from fifth grade children in the CARDIAC project and analyzed by local area hospitals or by LabCorp Inc. (Burlington, NC). Parents of participating children were sent a voucher for screening of their fasting blood lipid profile and reports were submitted to the CARDIAC project. Further details of the data collection procedure are described elsewhere. ${ }^{80-83}$ The West Virginia University Institutional Review Board (Protocol number 1504666639) approved merged analysis based on the child's identification number. We include only those observations where data was available from all three projects.

Matching Process. The WV Vital Statistics department (in Charleston, WV) sends the Birth Certificate data to the Birth Score project each month. This is done to ensure the number of Birth Score project forms completed by each hospital for every child born in the state. The Birth Score project data manager performed the matching process by linking maternal social security numbers using SAS software. The remaining unmatched babies are printed out and hand matched against the Vital Statistics Birth certificate data. The first and last name of the infant, birth hospital, mother's first, last, and maiden names, and date of birth are used to obtain the highest probable match.

This combined Birth Certificate/Birth Score data file was then used for linkage to the CARDIAC data. The CARDIAC office provided the CARDIAC data file, which consists of the 
CARDIAC identification number, the first and last name of the child, date of birth and the mother's name for years 2004-2010 ( $\mathrm{N}=46,198)$. The Birth Score applications programmer performed the matching process for all years. Overall, nearly $50 \%$ data match was achieved $(\mathrm{N}=22,136)$ between the CARDIAC and the Birth Score data. One of the main reasons for the unmatched data is the fact that not all children in the fifth grade in WV were necessarily born in WV and thus their information is not available in the Birth Score database.

\section{Variables}

Dependent variables. The main outcome variables for the study were available from the CARDIAC data, and include blood pressure (SBP and DBP) and lipid profile (TC, LDL, HDL, non-HDL, and TG) for the child and only lipid profile (TC, LDL, HDL, non-HDL, and TG) for the mother. Blood pressure was taken after the child had rested for five minutes. The first Korotkoff sound was used to record SBP and the fifth Korotkoff sound (K5, the last sound heard) was used to record DBP, measured in mm Hg. Lipids were both fasting and non-fasting, and measured in mg/dL; LabCorp estimated LDL using the Friedwald equation. All the outcomes were used as continuous variables. Some implausible values due to data coding error (e.g. SBP of $5 \mathrm{~mm} \mathrm{Hg}$ ) were set to missing. This included 8 observations for SBP, 28 observations for DBP, 10 observations for TC, 27 observations for LDL, 7 observations for TG, 5 observations for maternal TC, and 1 observation for maternal LDL.

Independent variable. The main exposure was defined as birth weight of the child in grams at the time of delivery. The continuous variable was obtained from the Birth Certificate data. The birth weight is also captured by the Birth Score Project with the following categories: $<1501$ grams, 1501-2000 grams, 2001-2500 grams, 2501-3000 grams, and >3000 grams. For the purpose of this project, we will use birth weight data for full-term infant only; i.e., for infants born $\geq 37$ weeks of gestation. This variable was used as both a continuous and a categorical variable depending on the type of analysis. Child's birth weight has been shown to be both linearly (positively ${ }^{72,73}$ and inversely ${ }^{33,38,74}$ ) and non-linearly (i.e., U-shape ${ }^{9,84}$ ) associated with childhood CVD risk factor measures. Because of this plausible U-shaped relationship, birth weight was categorized using conventional cut-offs categories as LBW $(<2,500$ grams), normal birth weight (2500-4000 grams), and HBW (>4000 grams).

Mediator. Trained area coordinators, nurses, and health science students measure the children's height and weight using SECA Road Rod stadiometer $(78 " / 200 \mathrm{~cm})$ and the SECA 840 Personal Digital Scale respectively (Seca Corp, Hanover, MD, USA). Body Mass Index (BMI) is a measure of weight adjusted for height and is calculated by CDC EpiInfo using the following 
equation: BMI $(\mathrm{kg} / \mathrm{m} 2)$ = weight $(\mathrm{kg}) /$ height $(\mathrm{m} 2)$. The study used BMI percentiles as a proxy for the child's current adiposity measure. ${ }^{85}$ The BMI for children ages 2 to 20 are plotted on a gender specific growth chart (the $2000 \mathrm{CDC}$ growth chart) ${ }^{86} \mathrm{BMI}$ percentiles are thus a measure of relative weight adjusted for the child's height, age and sex that corresponds to the $2000 \mathrm{CDC}$ growth charts. ${ }^{86}$

Covariates. We controlled for socio-demographic variables and other confounder variables that have shown to be associated with both the exposure (birth weight) and the outcomes of interest (CVD risk factors) or only with the outcomes of interest based on current literature.

Socio-demographic variables. The socio-demographic variables included the child's age, sex, race, maternal age, maternal race, education (at birth and later when child in fifth grade), and health insurance status at time of delivery. The age of the child in fifth grade was calculated from the CARDIAC screening date and parent-reported child birthdate in the CARDIAC project. The sex of the child was recorded at birth and at fifth grade by all three projects. This study used the sex (male or female), recorded by the CARDIAC project in fifth grade. The race/ethnicity of the child was parent-reported in fifth grade. For this study we dichotomized the race as "white" and "other" based on the population distribution of WV (i.e., 94\% white). ${ }^{87}$ The Birth Certificate as well the Birth Score Project captured data on maternal race, which was dichotomized as "white" and "other" as well.

Current literature shows inconsistent results of the association between maternal age and childhood CVD risk factors ${ }^{88,89}$ and between maternal age and maternal CVD risk factors. ${ }^{90,91}$ The association between maternal age at birth and childhood birth weight has been shown in numerous studies; ${ }^{92,93}$ thus maternal age (continuous) at the time of infant's birth was included as a confounder and recorded by the WV Birth Certificate data. For the maternal outcomes, the maternal age at birth was added to the child's age in fifth grade to generate maternal age when the child was in fifth grade. Current literature also shows that maternal socio-economic status such as maternal education and maternal health insurance are not only associated with giving birth to LBW babies, ${ }^{94-96}$ but are also associated with CVD risk factors in childhood, ${ }^{97}$ and with maternal CVD health as well. ${ }^{98}$ The maternal education at time of birth was recorded as a continuous variable and included the number of years of education received ranging from 1 to 17 . Maternal education was also recorded when the child was in fifth grade and was dichotomized as less than or equal to 12 grade and more than 12 grades of education. Maternal health insurance at the time of delivery was categorized as a binary variable (Medicaid and non-Medicaid). 
Family history of risk factors. The CARDIAC project collects information on family history of heart disease, coronary heart disease, heart attack, open-heart surgery, angioplasty, and death from heart disease; thus, we made a new variable called family history of CVD based on having family history of any one of these six variables. Family history of hypertension or high cholesterol is associated with having high blood pressure and abnormal lipid level in later life; ${ }^{99,100}$ the CARDIAC project collects information on family history of cholesterol (yes or no). Family history of diabetes (yes or no) was also available from the CARDIAC project.

Other infant and maternal characteristics. Additional covariates include number of previous pregnancies ( 0 or $\geq 1$ ), smoking during pregnancy (yes or no), smoking in the house when the child was in fifth grade (yes or no), weight gain during pregnancy (measured in pounds), gestational age (range 37 - 44 weeks) and infant feeding intention (breastfeed or both breastfeed and bottle). The literature shows that children from mothers with increasing parity (i.e., with one or more than one number of previous pregnancies; multiparous) have lower blood pressure, TC and LDL compared to children of mothers with no prior pregnancies (nulliparous). 101,102 There is also an association between nulliparity vs. multiparity and low birth weight. ${ }^{103}$ Furthermore, there is also an association between parity and maternal CVD risk. ${ }^{104,105}$

Smoking is a well-established risk factor for CVD. Maternal smoking during pregnancy has also been shown to be associated with childhood CVD risk factors ${ }^{106} 107$ as well as delivering LBW babies. ${ }^{108,109}$ Maternal smoking during pregnancy (yes or no) was obtained via self-report by the Birth Score project at birth. The CARDIAC project inquired if anyone smoked in the house when the child was in fifth grade as well. Current literature shows inconsistent association between gestational weight gain and higher risk of childhood CVD risk factors. ${ }^{110,111}$ Data on maternal gestational weight gain was available at birth and was assessed for its role as a covariate. Although this study only examined full-term birth infants (born $\geq 37$ weeks of gestation), children born full term can also have low birth weight; therefore the potential role gestational age (ranging from 37-44 weeks) as a confounder was assessed as well. The Birth Score Project collects information on mother's intention to breastfeed. Breastfeeding has shown to be protective for childhood CVD risk factors. ${ }^{112,113}$ Recent research also suggests that breastfeeding is significantly associated with positive CVD health outcomes for the mother as well. ${ }^{114-116}$ In our previous study we have demonstrated that intention to breastfeed is correlated ( $\mathrm{r}$ $=0.66$ ) with actual breastfeeding practices in WV children. ${ }^{117}$ Therefore, we used intent to breastfeed as a surrogate for actual breastfeeding practices. 


\section{Statistical Analysis}

All statistical analyses were conducted in SAS version 9.3 (SAS Institute, Cary NC). TG was $\log$ transformed in all analyses. The one-way analysis of variance (ANOVA) was used to determine whether there were significant differences between the means of all the outcomes of interest and three birth weight groups (LBW, normal birth weight, and HBW). Post hoc tests (i.e., Tukeys) were performed when there was significant omnibus F-tests to explore which means were significantly different from each other. Bivariate relationships between the birth weight (continuous) and the CVD risk factors (continuous variables) were assessed using Pearson correlation. Seven separate multiple-regression analyses were performed for the 7 continuous dependent variables (SBP, DBP, TC, LDL, HDL, non-HDL, and TG). All CVD outcomes were first regressed on birth weight (main independent variable) as a continuous variable as also as categorical variable using conventional cut-offs [LBW $(<2,500$ grams), normal birth weight (2500-4000 grams), and HBW (>4000 grams)] to determine for both the linear as well as the nonlinear relationship of birth weight and CVD risk factors (model 1). Two dummy variables were made for LBW and HBW categories and normal birth weight was utilized as the referent category. In separate models BMI percentile of the child in fifth grade was included in order to assess its role as a mediator (model 2). For all outcomes, additional covariates were added to model 2 (model 3). The decision to include the additional covariates was based on a priori hypotheses, existing literature, and associations that were found significant with the outcome variable at the bivariate level using Pearson (for continuous variables) or Spearman (for categorical variables) correlations. The covariates were dropped one at a time from the regression model if they were not significant (with highest $p$-value greater than $p>0.05$ ). However, if the main independent variable (birth weight) was not significant it was retained in the model regardless of its significance.

Ordinary Least Squares (OLS) regression was utilized, which is the most common type of multiple regression model where: $y=a+b x 1+b x 2+b \times 3+b x 4 \ldots b x 15$, where $y$ is the criterion DV, ' $a$ ' is the constant, ' $b$ ' is the slope weight values, and $x 1$ through $x 15$ are the values for the predictors (IVs). The slope ' $b$ ' gives us the change in the predicted value of $Y$, on average, for each unit increase in $\mathrm{X}$. Regression assumptions were assessed for all variables. The regression $\mathrm{F}$ statistic was used to determine the overall significance of the regression model and a corresponding $\mathrm{p}$-value of $\leq 0.05$ was used for statistical significant. The association of each independent variable to the outcome variable was also assessed for significance (alpha $\leq 0.05$ ) by examining the parameter estimates and their corresponding t-test values in addition to the $95 \%$ confidence intervals (CI) of the t-statistic. 
We report both standardized and unstandardized regression coefficients in order to assist in interpretation of the findings. The squared multiple correlations, $\mathrm{R}^{2}$, and adjusted $\mathrm{R}^{2}$ were interpreted as the effect sizes of the regression model (magnitude of the association). The study also performed the regression analysis with and without the main predictor variable (i.e., birth weight) in order to calculate the unique amount of variance shared between birth weight and CVD risk factors by calculating the change in $\mathrm{R}^{2}$ for outcomes assessed in model 3 only. Furthermore, the interactions between age and sex were also assessed and dropped from the model if they were not significant. (Note: the only significant interaction (age*sex) was present for LDL).

For the maternal CVD risk factors (TC, LDL, HDL, non-HDL, and TG), the same analyses were performed. The one-way analysis of variance (ANOVA) was used to determine whether there were significant differences between the means of all the outcomes of interest and three birth weight groups (LBW, normal birth weight, and HBW). Bivariate relationships between the birth weight (continuous) and the CVD risk factors (continuous variables) were assessed using Pearson correlation. Five separate multiple-regression analyses were performed for the 5 continuous dependent variables (TC, LDL, HDL, non HDL, and TG). If the bivariate relationships were significant, then multiple regression analysis was planned to regress the outcome variable on birth weight variable adjusting for additional covariates.

Sensitivity analyses. To explore whether there were gender differences we included the interaction term between gender and birth weight in the regression analysis. If the interaction term was significant we performed separate regression models by gender for that outcome. In order to use birth weight as a continuous variable and assess for the U-shape relationship, we used splines modeling technique that takes into consideration the relationship between the birth weight and the outcome within and between levels of the predictor variable (i.e. birth weight). ${ }^{118,119} \mathrm{~A}$ linear spline is a continuous function formed by connecting linear segments. They are piecewise polynomial segments in one variable of some degree $\mathrm{D}$ with function values, which is continuous and the function has D-1 derivatives that agree at the points where they join. The joining points are called knots that mark one transition to the next and allowing the curve the freedom to change direction and follow the data more accurately to model the relationship between the independent and the dependent variable. ${ }^{118,119}$ We used 2 knots at the two conventional cut-off points for birth weight distribution $(<2,500$ grams and $>4000$ grams $)$ and additional knots were also explored. This allows the slope of the regression to change at these two knots, thus allowing flexibility of the continuous birth weight to fit the non-linear segments (three segments). Lastly, we performed a post hoc sensitivity analysis by adjusting for pre-screening 
fasting levels in the regression analysis. Nearly $5 \%$ of the study population was not fasting before screening. Recent data shows that cholesterol levels test for fasting and non-fasting children are minor and do not have clinical relevance. ${ }^{140}$

\section{Results}

A total of 22,136 participants were available for analysis with the merged data. After excluding the infants born preterm (i.e. $<37$ weeks of gestation: $2,097(9.67 \%)$ ), the final number of participants for this study was 19,583. From the CARDIAC project data, the mean age of the children in fifth grade was $10.95( \pm 0.48), 56 \%$ were female, $95 \%$ infants were white. The mean BMI percentile of fifth graders was $73.05( \pm 27.84)$. Nearly $3 \%$ of the infants were LBW and $11 \%$ were HBW in full-term birth infants. The CVD risk factor data was available for approximately $85 \%$ of children. However, the maternal lipid data was available for only $6 \%$ of the mothers $(\mathrm{N}=1,121)$. The population characteristics of children and mothers are available in Table 1.

The means of all the outcomes of interest by the three birth weight groups (LBW, normal birth weight, and HBW) are given in Table 2. Only SBP and DBP showed significant overall results. Further exploration using post-hoc comparisons showed that the mean SBP and DBP for infants with HBW were significantly higher than infants born with normal birth weight. For the mothers, none of the mean lipid levels were significantly different for the three groups. Consistent with the ANOVA results, the correlations between birth weight (continuous) and SBP $(\mathrm{N}=19,397$, $\mathrm{r}=0.03, \mathrm{p}=0.0002)$, and between birth weight and DBP $(\mathrm{N}=19,344, \mathrm{r}=0.03, \mathrm{p}<0.0001)$, were significant and positive (Table 3). For the lipid levels, the correlations between birth weight and HDL ( $\mathrm{N}=16,066, \mathrm{r}=0.02, \mathrm{p}=0.0035)$ were significant and positive, and between birth weight and TG $(\mathrm{N}=15,951, \mathrm{r}=-0.03, \mathrm{p}=0.0005)$ were significant and negative. For the mothers there was significant and positive correlation of birth weight with maternal TC $(\mathrm{N}=1,116, \mathrm{r}=0.06, \mathrm{p}=$ 0.0386) only (Table 3).

The results of the multiple linear regression analysis using birth weight as a continuous variable showed the same pattern as observed in the correlations above for childhood CVD risk factors (Table 4a model 1). When using birth weight categories, only HBW was significantly higher than normal birth weight for child's SBP and DBP only and showed a trend towards significance for TC, LDL, and non-HDL cholesterol levels $(p=0.07)$ in children (Table 4a model 1). When the child's current BMI was added to the model the association between birth weight and SBP and DBP became non-significant. Moreover, the association between birth weight and SBP, TC, and LDL reversed but were not significant (Table 4a model 2). HDL was positively, 
and non-HDL and TG were negatively associated with the birth weight. Children born LBW had significantly lower HDL, and those born with HBW had significantly higher HDL levels, where as the reverse was true for non-HDL and TG in fifth grade (Table 4a model 2). Moreover, when additional covariates were adjusted for in the regression model, the association between continuous birth weight and LDL was significant with a $1.1 \mathrm{mg} / \mathrm{dL}$ decrease in LDL per $1000 \mathrm{~g}$ increase in birth weight $(b=-1.1 \mathrm{md} / \mathrm{dL} ;(95 \% \mathrm{CI}:-1.9,-0.2)$ per $1000 \mathrm{~g}$ increase in birth weight, $\mathrm{p}=0.02)$. The association between birth weight and non-HDL $[(b=-0.2 \mathrm{mg} / \mathrm{dL}(95 \% \mathrm{CI}:-0.3$, $0.1)$ per $1000 \mathrm{~g}$ increase in birth weight, $\mathrm{p}=0.0002)]$, and between birth weight and TG [( $\mathrm{b}=$ 0.005 (95\% CI: -0.007, -0.004) per $1000 \mathrm{~g}$ increase in birth weight, $\mathrm{p}=0.0001$ ] remained significant and negative (Table 4a model 3). For HDL the association remained significant and positively associated with birth weight in the fully adjusted model [ $(\mathrm{b}=0.1 \mathrm{mg} / \mathrm{dL}$ (95\% CI: 0.06 , 0.13 ) per $1000 \mathrm{~g}$ increase in birth weight, $\mathrm{p}=0.0001$ ) (Table $4 \mathrm{a}$ model 3 ). The list of additional covariates and their relationship with the outcomes are detailed in Table 5. The unique variance shared between birth weight and the significant outcomes were determined by calculating the change in R-square, which ranged from $0.02 \%$ to $0.2 \%$ (Table 5 ).

For the maternal lipid levels the unadjusted regression results showed that with every $1000 \mathrm{~g}$ increase in the child's birth weight the maternal TC increased by $0.5 \mathrm{mg} / \mathrm{dL}$ ( $95 \% \mathrm{CI}: 0.02$, 0.90) (Table $4 \mathrm{~b}$ model 1). However, the association became non-significant when adjustments were made for additional covariates $[(b=0.4 \mathrm{mg} / \mathrm{dL}(95 \% \mathrm{CI}:-0.01,0.87)$ per $1000 \mathrm{~g}$ increase in birth weight] (Table $4 \mathrm{~b}$ model 2).

No gender differences were observed in the additional sensitivity analysis that examined the interaction of birth weight by gender on blood pressure and lipid levels of children. The results of the sensitivity analyses that used spline regression models for children are presented in supplemental Tables S1. For the models that included the child's current BMI and additional covariates (Table S1-model 3) showed that with every one-unit increase in the birth weight the child's TC, LDL, and non-HDL levels decreased significantly in the $<2500$-gram segment only. DBP demonstrated a U-shaped relationship in both the crude and adjusted spline models. However, none of the segments were statistically significant. The results of the sensitivity analyses that used spline regression model for the mother data are presented in supplemental Table S2. The results were not significant for any of the spline birth weight segments and maternal lipids. The post hoc sensitivity analysis adjusting for children pre-screening fasting status showed no differences in the results of the multiple regression analysis that did not adjust for the pre-screening fasting status of the child. 


\section{Discussion}

Overall, the results showed a linear positive association between birth weight and maternal TC levels and between birth weight and childhood SBP, DBP, and HDL and a negative association of birth weight with child's TG at the bivariate level (based on the results of the Pearson correlation and linear regression (unadjusted) results). When adjusted for the child's current BMI percentile in fifth grade, the association between birth weight and SBP and DBP became non-significant. However, the association between birth weight and HDL and TG not only remained significant but also strengthened. Additionally, non-HDL was negatively but not significantly associated with birth weight in the crude analysis, and became significantly and negatively associated with birth weight after adjusting for the child's current BMI. Surprisingly, when additional covariates were added to the models, the association between birth weight and LDL also became significant, demonstrating a negative association as well.

\section{Blood Pressure in Children}

Our results are consistent with studies that found a positive association between birth weight and childhood SBP for the crude analysis that reversed in direction for the adjusted association. ${ }^{45,120}$ Although the direction of our study is consistent with previous studies, unlike most studies the adjusted association was not significant. ${ }^{34,36,40,68,121}$ However a recent study that examined this association in WV fifth grade found no significant differences in SBP in fifth grade by gestational group (SGA, AGA, LGA) or comparing preterm to term birth infants. ${ }^{47}$ As our study focused on term birth infants only, it is difficult to compare the findings to the earlier work done on WV fifth grade children. The results of our study suggest that the association between birth weight and childhood is potentially mediated by the child's current BMI. The direction of the association between birth weight and SBP is consistent with the Barker's hypothesis for the adjusted analysis only. However, the non-significant findings suggest that the association is not independent of the child's current BMI.

Our results are similarly consistent with studies that found a significant positive association between birth weight and childhood DBP for the unadjusted analysis ${ }^{37,120}$ that became non-significant (but remained positive) after adjusting for the child's current BMI. ${ }^{42}$ Azadbakht and collegues also found significant mean differences in DBP levels according to birth weight categories and also a positive crude association as observed in our study. However unlike the results of our study their findings remained signifiant after adjusting for child's current BMI, ${ }^{9}$ as

demostrated by other studies as well. ${ }^{33,36}$ Our study demonstated a significant positive linear 
association between birth weight and DBP $(b=0.06 \mathrm{mmHg}$; 95\% CI: $0.03-0.08 ; \mathrm{p}<0.0001$ for $1,000 \mathrm{~g}$ increase in birth weight) that attenuated and became non-significant when adjusted for the child's current BMI $(b=0.01 \mathrm{mmHg}$; 95\% CI: $-0.01,0.04 ; \mathrm{p}=0.4916$ for $1,000 \mathrm{~g}$ increase in birth weight). The results from the spline regression models (sensitivity analysis) for DBP demonstrated a U-shaped relationship in both the crude and adjusted spline regression models. However, none of the segments were statistically significant. The direction of the association between birth weight and DBP is not consistent with the Barker's hypothesis for both the crude and the adjusted analyses. However, it is important to note that DBP is a less reliable measure compared to SBP in children. ${ }^{122,123}$ Furthermore, numerous studies have shown SBP to be a stronger predictor of CVD compared to DBP. ${ }^{124}$

\section{Lipids in Children}

Our results are consistent with studies that have found a significant association between birth weight and childhood HDL (positive), ${ }^{9,34}$ and between birth weight and TGs (negative). ${ }^{35}$ Adjustments for the child's current BMI strengthened the relationship between birth weight and HDL and between birth weight and TG levels in children. LDL and non-HDL were not significant in the crude analysis but became significant and negatively associated with birth weight after accounting for the child's BMI and additional covariates. Our results for non-HDL and LDL are consistent with the findings of other studies as well. ${ }^{34,36}$ For TC; our results are not consistent with the systematic review that found a weak inverse relationship between birth weight and childhood TC levels. ${ }^{125}$ Another study that used WV CARDIAC data for 11 years old children found that infants who were born preterm had higher levels of TGs compared to term infants. The study also found that SGA infants were less likely to be obese in fifth grade, but those who were obese had higher TG levels compared to AGA or LGA obese children. ${ }^{47}$ The study did not find an association with other lipid levels. However, the population in our study was limited to term infants only, which differed from the study by Mullett and colleagues that included preterm infants as well. ${ }^{47}$

Our results demonstrated that LBW was associated with lower mean HDL and higher LDL, non-HDL, and TG levels independent of the child's current BMI and additional covariates. When birth weight was categorized, LBW vs. normal birth weight subjects had higher LDL and non-HDL levels, and HBW subjects had higher HDL and lower TG compared to normal birth weight children. These results suggest that association between birth weight and lipid levels is linear and not U-shaped as demonstrated by some studies. ${ }^{35,84,126}$ Based on these results, we hypothesize that the association between birth weight and lipids is independent of the child's 
current BMI levels. However, the change in $\mathrm{R}^{2}$ showed that birth weight accounted for less than $1 \%$ of unique variance in lipid levels of fifth grade WV children, suggesting a small effect size. Interpreting significant covariates included in the study may be of some interest as well (Table 5). The results suggest that girls had significantly higher LDL, higher TG and lower HDL compared to boys as observed in other studies as well. ${ }^{127,128}$ The interaction between sex and age was significant for LDL only, which suggested that there was an increase in LDL levels with an increase in age and the increase was much larger for girls than for boys. Although the age range in our study was only 5 years (9 to 14), we observed that there was a significant increase in the level of TGs ${ }^{129}$ and a decrease in HDL $^{130}$ and non-HDL with age during childhood as observed in other studies as well. ${ }^{131}$ Race (white vs. others) was a significant factor for HDL (lower) and TG (higher) only, which is also consistent in the literature. ${ }^{129,132}$ Family history of CVD or cholesterol was positively associated with the child LDL, non-HDL, and TG levels and negatively associated with HDL as expected based on current literature. ${ }^{99,100,133}$ Previous literature has shown inconsistent association between maternal education and child's TG levels, ${ }^{134,135}$ our study demonstrates that the increase in mothers' education at birth was associated with lower child's TG levels $(b=-0.01 \mathrm{mg} / \mathrm{dL}$ decrease in TG per 1 year increase in maternal education, $95 \% \mathrm{CI}$ : $0.02,-0.01)$. Maternal age at time of birth was not significantly related to childhood lipid levels as observed in other studies as well, ${ }^{89}$ except for HDL levels, which increased as the maternal age at birth increased. Maternal smoking during pregnancy was associated with lower HDL during childhood as observed in another study examining lipid level in 8-year-old children. ${ }^{106}$ However, previous literature has shown this association with other lipid levels as well ${ }^{106,107}$ not observed in this study. Mother who intended to 'breastfeed only' had children with significantly lower nonHDL compared to mothers who 'intended to breastfeed and bottle-feed' their infants. Lastly, and most importantly the BMI percentile was significantly associated with all lipid levels. The standardized regression coefficients for the outcomes ranged from 0.14 to 0.35 (negative for HDL) for every one unit standardized increase in the BMI percentile of children (Table 5).

\section{Adjusting for Current BMI}

Our study results showed that the positive association between birth weight and SBP, TC, and LDL became negative (reversed) when current BMI was added in the model. However the change in direction from positive to negative after adjusting for current BMI is an area of great controversy. Some believe that adjusting for current BMI may produce spurious inverse association. ${ }^{136,137} \mathrm{Tu}$ and colleagues performed a simulation study where the true positive association between birth weight and adult blood pressure attenuated and even reversed when the 
correlation between birth weight and the current weight was increased. ${ }^{137}$ However, Gillman and colleagues demonstrate that adjusting for current body size is not only appropriate but can also help us understand the inverse birth weight and CVD risk factors association. ${ }^{138}$ The systematic review by Lauren and colleagues concluded that there was no consistent relationship between birth weight and later lipid levels and most studies that adjusted for the current size were the ones that supported the Barkers hypothesis only. ${ }^{84}$ Other researchers have also recommended not adjusting for current body size. ${ }^{39,69}$ Menezes and colleagues found the positive association between birth weight and blood pressure that reversed direction after adjusting for current BMI. However the authors state that regardless of their findings they do not support the inverse association because birth weight adjusted for current weight is a proxy measure of post-natal growth and not a measure of birth weight. ${ }^{120}$ In our study the direction of association between birth weight and SBP, TC, and LDL changed from positive to negative when adjusted for current BMI. However, based on our unadjusted results (model 1) we can clearly see that LBW was significantly associated with higher TG and lower HDL levels and adjustment for current BMI only strengthened the associations. Overall, our results suggest that BMI is a key predictor of CVD risk factors (Table 5) and a potential mediator for the relationship between birth weight and CVD risk factors and therefore should be accounted for in the regression models. (Note in our study: Pearson correlations between BMI and birth weight $(r=0.10)$, SBP $(r=0.34)$, DBP $(r=$ $0.26), \mathrm{TC}(\mathrm{r}=0.10), \operatorname{LDL}(\mathrm{r}=0.15), \operatorname{HDL}(\mathrm{r}=-0.33)$, non-HDL $(\mathrm{r}=0.23)$, and $\log -\mathrm{TG}(\mathrm{r}=$ $0.35), \mathrm{p}<0.0001)$.

\section{Lipids in Mothers}

The results of our study found no significant mean difference in any of the lipid levels of mothers by birth weight categories of the child. However, the correlation between birth weight and maternal TC was significant and positive. The nature of the association was further revealed when the birth weight was categorized in the regression analysis, which demonstrated that mothers who gave birth to HBW babies had higher TC compared to mothers who gave birth to normal birth weight babies. However, the association became non-significant when additional covariates were added in the model. None of the other lipid levels were significant associated with the birth weight of infant. Although, not significant the direction of association suggests that mothers who give birth to HBW babies have a trend towards increased risk of poor lipid levels 11 years post-partum as demonstrated by a positive association between birth weight and TC, LDL, non-HDL, TG and a negative association with HDL. A study by Yajnik and colleagues found no significant relationship between the infant birth weight and maternal TC, LDL, and HDL but 
found significant positive association for TG levels in India. ${ }^{58}$ Our results are consistent with Yajnik et al., findings for the direction of association between birth weight and lipid levels but not significant for TG as found in this earlier work. ${ }^{58}$ This may be due to differences in the birth weight distribution of the two populations. Furthermore, we did not have information on maternal lipid levels or maternal BMI, which has been associated with infants birth weight. ${ }^{139} \mathrm{We}$ also did not have information on the birth weight of the mothers when they were born. Whether mother's abnormal lipid levels are associated with giving birth to low or high birth weight babies, or whether the birth weight of the infant is a potential risk factor for maternal lipid level in later years, or whether the mother's own birth weight impacts their lipid profile is difficult to establish at this point. The research on this topic is still in its infancy and needs further exploration.

\section{Limitations of the Study}

Some of the limitations of the study include lack of information on possible important confounder variables such as parental adiposity status, maternal pre-pregnancy weight, maternal lipid status before and during pregnancy, maternal birth weight, rapid weight gain during the first year of life, pubertal status of the child, family history of hypertension, physical activity, and dietary behaviors. Furthermore, the CARDIAC project only collects parental lipid data and thus the maternal CVD risk factors were limited to lipid profile only, which was available for a small sample of the population. Only a small percent of mothers of participating CARDIAC students took advantage of the free vouchers for lipid testing, thus limiting the generalizability of the sample. Due to the unique characteristics of the Appalachian state of WV, results may not be generalizable beyond the participants included in this study.

\section{Strengths of the Study}

Despite these limitations, this study demonstrates a significant linear association between birth weight and child's lipid levels in fifth grade using a large longitudinal dataset with information available for important covariates. The direction of association of all outcomes (except DBP) was consistent with the Barker's hypothesis. To our knowledge this is the first study that has examined these associations in the state of WV for both mothers and the children born full-term at birth. These results provide an important contribution for expanding the research examining the association between birth weight of the infant and maternal CVD health. 


\section{Conclusion}

Our earlier work has shown that HBW is associated with higher childhood BMI in fifth grade WV children. ${ }^{117}$ This study provides information on the relationship between birth weight and other CVD risk factors as well, demonstrating that LBW is associated with higher risk of abnormal lipid levels (higher LDL, non-HDL, and TGs and lower HDL levels) in fifth grade children independent of current weight status in this young Appalachian population. As there is tracking of CVD risk factors from childhood into adulthood, there is a potential that these small effect sizes can have detrimental effect on CVD in later adulthood in WV. For the mothers CVD risk factors, HBW of the infant seemed to demonstrate a trend towards poor maternal lipid levels 11 years post-partum. Well-designed longitudinal studies are needed to understand the complex biological pathways for both the mother and the child and examine these associations at different stages of life. Furthermore, future research should also focus on working with diverse populations to establish different birth weight cut-offs that can increase the sensitivity of identifying infants

with higher risk of future CVD as suggested by the recent systematic review as well. ${ }^{44}$ 


\title{
Chapter 3: Breastfeeding and childhood and maternal cardiovascular risk factors
}

\begin{abstract}
Introduction: The Appalachian state of West Virginia (WV) has a higher prevalence of cardiovascular disease (CVD) and lower breastfeeding rates compared to the national averages. There is paucity of research examining the relationship of breastfeeding to subsequent childhood and maternal CVD risk factors, an issue of particular relevance in this rural state.

Methods: The study used longitudinally linked data from three cross-sectional datasets in WV datasets [West Virginia Birth Certificates, the Working in Appalachia to Track High Birth Score, Critical Congenital Heart Disease and Hearing Loss (WATCH)/Birth Score project, and the Coronary Artery Risk Detection in Appalachian Communities (CARDIAC) project]. The information on the main exposure "history of breastfeeding" was obtained retrospectively via parental recall when the child was in fifth grade. The outcome variables included blood pressure measures (systolic blood pressure (SBP), diastolic blood pressure (DBP)) for children and lipid profile for both the mother and child (total cholesterol (TC), low-density lipoprotein cholesterol (LDL), high-density lipoprotein cholesterol (HDL), non-high-density lipoprotein cholesterol (non-HDL), and triglycerides (TG)). Mean differences, correlations, and simple regression analyses were performed to examine the crude associations. Multiple regression analysis was performed adjusting for current body mass index (BMI) and additional covariates.

Results: The final study sample after excluding infants born pre-term (i.e. $<37$ weeks of gestation: $\mathrm{N}=1,190,10.2 \%$ ) was 10,457 . Nearly, $43 \%$ of the mothers self-reported breastfeeding their index child. The results showed a significant negative correlation between reported history of breastfeeding and childhood SBP $(\mathrm{r}=-0.06)$, DBP $(\mathrm{r}=-0.04)$, and TGs $(\mathrm{r}=-0.07)$, and a positive correlation between breastfeeding history and child's HDL levels $(r=0.04)$. When adjusted for the child's current BMI and socio-demographic variables in the regression analysis, the association was statistically significant for TGs only (beta $=-0.04 \mathrm{mg} / \mathrm{dL} ; 95 \% \mathrm{CI}$ : -0.06 , $0.01 ; p=0.01$ ). Maternal mean lipid levels were not significantly different between the two groups nor were significantly correlated to their reported history of breastfeeding.

Conclusion: The observed protective effect of breastfeeding on the child's TGs level in fifth grade was small but significant. This supports the promotion of breastfeeding as a possible preventive measure for future CVD risk factors in the rural Appalachian state of WV.

Nonetheless future research should focus on designing large prospective studies to assess and understand the association of breastfeeding with both maternal and child CVD risks factors at various life stages.
\end{abstract}




\section{Introduction}

Cardiovascular disease (CVD) mortality accounts for nearly $50 \%$ of mortality, or 17.5 million out of 38 million chronic disease mortality worldwide. ${ }^{1,2}$ CVD is also the leading cause of mortality and morbidity in the United States (U.S.) ${ }^{3}$ with nearly one third of adults having at least one type of CVD. ${ }^{4,5}$ CVD mortality rates are nearly $20 \%$ higher in the rural Appalachian population compared to the rest of the nation. ${ }^{6}$ West Virginia (WV), a state entirely within the Appalachian region, has one of the highest rates of CVD mortality (ranks 45) in the nation and also one of the highest prevalence of CVD risk factors ranking number 50th in high blood pressure and 48th in high total cholesterol level. ${ }^{7}$ Data on early life determinants of CVD risk suggests that breastfeeding is protective for cardiovascular health of an individual and also has a protective effect on mothers who choose to breastfeed their infant. ${ }^{8-11}$

American Academy of Pediatrics (AAP) suggests six months of exclusive breastfeeding and continuation of breastfeeding until the infant is one year old while gradually introducing solid foods after six months. ${ }^{12}$ However, results from the 2011 National Immunization Survey (NIS) show that only $18.8 \%$ of mothers in the U.S. and $12.2 \%$ of the mothers in WV exclusively breastfeed for the first six months. The percentage of mothers who breastfeed but not exclusively for the first six months postpartum is also much lower in WV (29\%) compared to the national average of $49 \%{ }^{13}$ Pediatric Nutrition Surveillance System (PedNSS), is another survey that monitors nutritional status of low-income children enrolled in federally funded maternal and child health program (mainly WIC). The results from 2011 PedNSS shows the only $13.5 \%$ of women in WV enrolled in these programs breastfeed for six months where as only $0.3 \%$ exclusively breastfed their infant. The results from 2011 NIS and PedNSS survey for the state of WV as well as national rates are shown in Table $1 . .^{13,14}$

Bartick and colleagues performed a pediatric cost analysis and concluded that the cost of morbidity and mortality associated with suboptimal breastfeeding in the U.S. was approximately $\$ 14$ billion annually in 2011..$^{15,16}$ The researchers also performed a cost analysis of maternal morbidity and mortality associated with suboptimal breastfeeding. The study calculated the cost to be approximately $\$ 17$ billion from premature deaths and $\$ 733.7$ million (direct) and $\$ 126.1$ million (indirect) morbidity costs. ${ }^{17}$

The detailed literature review presented below outlines the associations between breastfeeding and child CVD risk factors including blood pressure (systolic blood pressure (SBP), diastolic blood pressure (DBP)), and lipid profile [(total cholesterol (TC), low-density lipoprotein cholesterol (LDL), high-density lipoprotein cholesterol (HDL), non-high-density lipoprotein 
cholesterol (non-HDL), and triglycerides (TG)], and then expands on associations between mothers who breastfeed their infant and maternal CVD risk factors.

\section{Breastfeeding and Childhood CVD Risk Factors}

Breastfeeding has shown to be protective against childhood obesity. ${ }^{18}$ Data shows that the risk of childhood obesity decreases (ranging from 15\% to 35\%) significantly in children who were breastfed compared to children who were not breastfed. ${ }^{19-21}$ In addition to childhood adiposity, breastfeeding has been shown to have protective effects on other CVD risk factors during childhood such as blood pressure, cholesterol, and diabetes. ${ }^{22}$

A study that examined the association between breastfeeding duration and child's blood pressure at 8 years of age found significant higher mean values of SBP (but not for DBP) for children who were breastfed for $<40$ days ( $99 \mathrm{mmHg}$ ) compared to children who were breastfed for $\geq 40$ days $(93 \mathrm{mmHg})^{23}$ suggesting a dose-response relationship between breastfeeding duration and later childhood blood pressure. ${ }^{24}$ In another study, SBP was $1.19 \mathrm{mmHg}$ (95\% CI: $0.40,1.96)$ lower among 5 -year-old children who were breastfed for at least 6 months compared to those who were not, even after adjusting the child's current weight status. ${ }^{25}$ In another larger prospective cohort study, SBP was $1.2 \mathrm{mmHg}$ lower $(95 \% \mathrm{CI}: 0.5,1.9)$ and DBP was $0.9 \mathrm{mmHg}$ lower $(95 \%$ CI: $0.3,1.4)$ among breastfed children, compared with children who were never breastfed after adjusting for covariates. ${ }^{26}$ Consistent with these results, a systematic review with meta-analysis found the pooled mean difference in SBP was $-1.10 \mathrm{mmHg}(95 \% \mathrm{CI}:-1.79,-0.42)$ among infants who were breastfed during infancy but DBP was not significantly related to the type of infant feeding. ${ }^{27}$ Another systematic review on the topic showed that breastfeeding was associated with a $1.4 \mathrm{mmHg}$ reduction in SBP and $0.5 \mathrm{mmHg}$ reductions in DBP in later life. ${ }^{28}$

However, other studies have shown no association between blood pressure and breastfed and non-breastfed children, ${ }^{29,30}$ or between breastfeeding duration and blood pressure in adolescence and young adults. ${ }^{31-34}$ For example, results from a randomized controlled trial for a breastfeeding promotion intervention found no differences in adiposity or blood pressure at age 6.5 years in the experimental group compared to the control group. ${ }^{35,36}$ In an observational study, neither exclusive nor partial breastfeeding (compared to never breastfeeding) were significantly associated with blood pressure in 13-year-old adolescents. ${ }^{30}$ Furthermore, a recent systematic review also found no association between breastfeeding and blood pressure in children ages 10$19 .{ }^{37}$

Breastfeeding has also been studied in relation to childhood and adolescence cholesterol levels. ${ }^{22}$ A study found that cholesterol concentration was significantly lower in boys (but not in 
girls) who were breastfed for a median duration of $<2$ months compared to those who were formula fed. ${ }^{38}$ Another study that examined the association between breastfeeding and BMI and serum lipids concentrations at 6 years, found that boys who were breastfed for $<6$ months had significantly higher BMI than those breastfed for $>8$ months and longer duration was also related to higher HDL levels in girls but not in boys. ${ }^{39}$ These results may also be beneficial later in life: one study demonstrated a significant association between breastfeeding and higher levels of TC and LDL cholesterol in older women compared to men. ${ }^{40}$ Two systematic reviews on the topic also found breastfeeding to be associated with decreased risk of blood cholesterol levels later in child's life ${ }^{22}$ or in adolescence and adult life. ${ }^{41}$

Although generally the literature notes a positive protective factor of breastfeeding against abnormal lipid profiles, other studies showed no association between breastfeeding and serum lipid concentrations in children ${ }^{42}$ or in adults. ${ }^{34}$ But even these results may be misleading due to the time in which the lipid measurements were taken. For example, two studies found no difference in mean TC and LDL levels between breast feeders and bottle feeders in adolescence (ages 13-16 years), but demonstrated that breastfeeding was associated with higher TC and LDL during infancy ( $<1$ years) and lower mean TC and LDL levels in adults $>17$ years even after adjustment for BMI. ${ }^{41,43}$ Moreover, some studies have found the negative association with one lipoprotein but not with the other types of lipoproteins or only in one of the sexes. For example, in a population based cohort study in Brazil, the LDL cholesterol was higher among never (mean $41.0 \mathrm{mg} / \mathrm{dl} ; 95 \%$ CI: 39.4, 42.7) than among ever breast fed adolescent boys $(38.6 \mathrm{mg} / \mathrm{dl} ; 95 \%$ CI: 38.6, 40.3), while the associations with all other lipoprotein concentrations were not significant. ${ }^{44}$ Another study of 17 -year-old adolescents found significantly higher TG levels in boys only and the association was not significant for girls and for other lipoprotein

concentrations. ${ }^{45}$ Additionally, a recent systematic review with meta-analysis found no association between breastfeeding and TC levels for all age groups including ages 10-19. ${ }^{37}$

\section{Breastfeeding and Maternal CVD Risk Factors}

Recent research suggests that breastfeeding is significantly associated with positive cardiovascular health not only for the child but also for the mother. ${ }^{46-48}$ A recent study examining the association between lactation and CVD mortality found that parous women $<65$ years who had never breastfed had a higher CVD mortality than women who had breastfed 24 months or more (Hazard ratio (HR): 2.77, 95\% CI: 1.28, 5.99). ${ }^{49}$ Another study noted that women who breastfed for a lifetime total of $>2$ years had a $23 \%$ lower risk of heart disease compared to women who had never breastfed. ${ }^{11}$ The researchers also found a dose-response relationship 
between duration of lactation and lower prevalence of hypertension and hyperlipidemia in postmenopausal women (median age 63 years) even after adjusting for the maternal BMI. ${ }^{11}$ Thus, the beneficial effect of breastfeeding for mothers may extend to CVD risk factors such as metabolic syndrome, obesity, hypertension, and high cholesterol. For example, a cross-sectional study demonstrated that women who breastfed had significantly lower odds of metabolic syndrome in later life $(\mathrm{OR}=0.79,95 \% \mathrm{CI}: 0.63 ; 0.99) .{ }^{48}$ Another study also found that women who breastfed for $<12$ weeks had significantly greater weight gain over a 15 year follow up period compared to women who breastfed for $>12$ weeks. ${ }^{50}$ Other researchers note that breastfeeding lowered maternal blood pressure as early as one month to five months postpartum ${ }^{10,51}$ and continued to have beneficial effects later in life (45-64 years of age). ${ }^{52}$ On average, women who breastfed for $<3$ months have higher visceral fat content, ${ }^{53}$ along with higher TC and TG levels compared to women who breastfed their children for at least three months.$^{54}$ However, McClure et al., noted that the association between breastfeeding and HDL became non-significant after controlling for socio-demographic variables. ${ }^{54}$

Conversely, a randomized controlled trial showed that longer breastfeeding duration did not lower maternal adiposity or blood pressure 11.5 years postpartum ${ }^{55}$ and a prospective cohort study did not find an association between breastfeeding duration and any of the serum lipid markers (TC, TG, HDL, and LDL) at 3 years postpartum. ${ }^{56}$

\section{Biology of Breastfeeding and CVD}

Children. The composition and constituents of human milk are specifically intended for human babies and any other form of feeding preparations is markedly different from human milk. ${ }^{57,58}$ One of the main differences in human milk and formula milk is the fat content, which differs throughout the day and within a single milk expression, as well as across each stage of the infant's growth. ${ }^{59}$ Formula milk is not responsive to the nutritional needs of the infant as the infant ages. ${ }^{60}$ Human milk also contains digestive enzymes that help in the digestion of milk and absorption of fat. ${ }^{61}$ This enzyme is absent in formula milk, which makes the digestive process difficult. Some researchers hypothesize that the differences in health outcomes observed in children are due to the differences in the infant's digestion of human milk or formula milk. ${ }^{62}$ Other researchers note that the fat content in human milk may provide satiety signal, which protects against overfeeding, in addition to the assumption that breastfed infants better learn to regulate the amount of milk consumed. ${ }^{63-65}$ Studies have shown that breastfeeding increases the infant's ability to self-regulate energy intake, which prevents them from overconsumption. ${ }^{67,68}$ In addition to digestive enzymes, human milk also contains growth factors or hormones that could 
also have an effect on the lipid metabolism and growth and development of the infant. ${ }^{66}$ Lastly, Li and colleagues examined the satiety responsiveness of 6 year old children found that infants who were bottle fed had low satiety responsiveness compared to breastfed infants, probably due to parental control in infancy to empty their bottles. ${ }^{69}$

Mother. Several experts in the field have summarized the biological explanation for the association between lactation and maternal lipid levels. During pregnancy, lipid levels increase and after delivery these levels decrease rapidly in lactating women. This is potentially due to alteration in lipid metabolism, as lactation process requires mobilization and redistribution of lipids from body fat storage for milk production. ${ }^{48,70}$ Lactation not only increases the mother's metabolic expenditure (nearly $500 \mathrm{kcal} /$ day), ${ }^{71}$ the process also improves the metabolic system by making it more efficient. ${ }^{11,48,72}$ This metabolic efficiency can potentially reverse the gestational increases in fat and lipid accumulation and have a long-term positive impact on women's cardiovascular health. ${ }^{70}$ The decrease in lipid levels have been observed as early as 6 to 13 weeks after delivery and up to three years postpartum; others have noted these differences even postmenopause. ${ }^{11,73-75}$

\section{Statement of the Problem}

In summary, the literature examining the association between breastfeeding and later childhood CVD risk presents some inconsistent results. While most studies have found a significant protective effect of breastfeeding on child CVD risk factors ${ }^{23}$ others have not. ${ }^{30,35,41,43}$ Data shows that childhood adiposity is associated with blood pressure and cholesterol. ${ }^{76-78}$ While some studies have controlled for childhood obesity and found that the association between breastfeeding and blood pressure and lipid levels attenuates, ${ }^{42}$ others have found that even after controlling for BMI, the association remains significant. ${ }^{23}$ These conflicting results suggest that childhood obesity could potentially be a partial or a full mediator between the observed effects. Furthermore, some studies have found this association to be gender specific, ${ }^{38,39,45}$ age specific (adults only), ${ }^{40}$ or specific to one or two components of CVD risk factors. ${ }^{44,45}$

The association between maternal breastfeeding and maternal CVD risk factors also show inconsistent results. Some studies suggest that mothers who breastfeed have lower CVD risk factor in later life, ${ }^{11,50,52}$ while others did not find a significant association. ${ }^{55,56}$ Most importantly, to the best of our knowledge, we did not find any study examining these associations longitudinally in the state of WV; where focusing on improving the low breastfeeding rates in the state could potentially have an impact on the future CVD burden. The purpose of this study was to investigate the influence of infant breastfeeding and childhood CVD risk factors (SBP, DBP, 
TC, LDL, HDL, non-HDL, and TG) at 11 years of age (controlling for child's current BMI), and the subsequent risk of developing maternal CVD risk factors (TC, LDL, HDL, non-HDL, and TG) 11 years post partum.

\section{Methods and Materials}

\section{Data Sources.}

The study used data from three projects, including WV Birth Certificates, the Working in Appalachia to Track High Birth Score, Critical Congenital Heart Disease and Hearing Loss (WATCH)/Birth Score project, and the Coronary Artery Risk Detection in Appalachian Communities (CARDIAC) project. The WATCH/WV Birth Score project is an infant riskscreening instrument that was initiated in $1985 . .^{79,80}$ Trained health care professionals collect data on every newborn within 24-48 hours or prior to discharge, in order to identify infants who are at a high risk of poor health outcomes or mortality in the first year of life, for referral to primary care management. ${ }^{79-81}$ In this study, children participating in the Birth-Score project (all of whom are merged with the Birth Certificate data) born between in 1997 to 2004 were merged with data collected by the CARDIAC Project in years 2008-2014. The CARDIAC project collects data on fifth grade public school children in 55 counties in WV with informed consent by parents/guardians and assent by the child. ${ }^{82}$ Area coordinators employed by the project, along with health science student volunteers, local school nurses, and volunteer phlebotomists conduct blood pressure, anthropometric measurements, and blood lipid testing. Blood sample is obtained from fifth grade children in the CARDIAC project and analyzed by local area hospitals or by LabCorp Inc. (Burlington, NC). Parents of participating children were sent a voucher for screening of their fasting blood lipid profile and reports were submitted to the CARDIAC project. Further details of the data collection procedure are described elsewhere. ${ }^{83-86}$ The WV University Institutional Review Board (Protocol number 1504666639) approved the merged analysis based on the child's identification number. We include only those observations where data was available from all three projects.

Matching Process. The WV Vital Statistics department (in Charleston, WV) sends the Birth Certificate data to the Birth Score project each month. This is done to ensure the number of Birth Score project forms completed by each hospital for every child born in the state. The Birth Score project data manager performed the matching process by linking maternal social security numbers using SAS software. The remaining unmatched babies are printed out and hand matched against the Vital Statistics Birth Certificate data. The first and last name of the infant, birth 
hospital, mother's first, last, and maiden names, and date of birth are used to obtain the highest probable match.

This combined Birth Certificate/Birth Score data file was then used for linkage to the CARDIAC data. The CARDIAC office provided the CARDIAC data file, which consists of the CARDIAC identification number, the first and last name of the child, date of birth and the mother's name for years 2008-2014 ( $\mathrm{N}=20,531)$. The Birth Score applications programmer performed the matching process for all years. Overall, nearly $60 \%$ data match was achieved $(\mathrm{N}=11,980,58.4 \%)$ between the CARDIAC and the Birth Score data. One of the main reasons for the unmatched data is the fact that not all children in the fifth grade in WV were necessarily born in WV and thus their information are not available in the Birth Score database.

\section{Variables.}

Dependent variables. The main outcome variables for the proposed study are available from the CARDIAC data, and include blood pressure and lipid profile (SBP, DBP, TC, LDL, HDL, non-HDL, and TG) for the child and lipid profile for the mother (TC, LDL, HDL, nonHDL, and TG). If maternal lipid profile was available for stepmother, adoptive-mother or legal guardian, the observations were excluded for the maternal analyses only. Blood pressure was taken after the child had rested for five minutes. The first Korotkoff sound was used to record SBP and the fifth Korotkoff sound was used to record DBP, measured in mm Hg. Lipids were both fasting and non-fasting, and measured in $\mathrm{mg} / \mathrm{dL}$; LabCorp estimated LDL using the Friedwald equation. All the outcomes were used as continuous variables. Some implausible values due to data coding error (e.g. SBP of $0 \mathrm{~mm} \mathrm{Hg}$ ) were set to missing. This included 2 observations for SBP and 1 observation for DBP.

Independent variable. The main exposure variable was the reported history of breastfeeding. Information related to breastfeeding was obtained using the CARDIAC questionnaire retrospectively via parental/caregiver recall when the child was in fifth grade. The question stated, "Was your child breastfed?" and the answer options included 'yes', 'no' and 'don't know'. The 'don't know' option was recoded to missing.

Mediator. Trained area coordinators, nurses, and health science students measure the children's height and weight using SECA Road Rod stadiometer $(78 \% / 200 \mathrm{~cm})$ and the SECA 840 Personal Digital Scale respectively (Seca Corp, Hanover, MD, USA). Body Mass Index (BMI) is a measure of weight adjusted for height and is calculated by CDC EpiInfo using the following equation: BMI $\left(\mathrm{kg} / \mathrm{m}^{2}\right)=$ weight $(\mathrm{kg}) /$ height $\left(\mathrm{m}^{2}\right)$. The study used BMI percentiles as a proxy for the child's current adiposity measure. ${ }^{87}$ The BMI for children ages 2 to 20 are plotted on a gender 
specific growth chart (the $2000 \mathrm{CDC}$ growth chart) ${ }^{88}$ Established cut-off points were used to identify underweight (less than 5th percentile); healthy weight (5th to 84.9th percentile); overweight (85th to 94.9th percentile); obese (95th to 98.9th percentile); and morbidly obese (greater than 99th percentile) weight categories. ${ }^{89}$ BMI percentiles are thus a measure of relative weight adjusted for the child's height, age and sex that corresponds to the $2000 \mathrm{CDC}$ growth charts. $^{88}$

Covariates. We controlled for socio-demographic variables and other confounder variables that have shown to be associated with both the exposure (breastfeeding) and the outcomes of interest (CVD risk factors) or only with the outcomes of interest based on current literature.

Socio-demographic variables. The socio-demographic variables included the child's age, sex, race, maternal age, education and health insurance status at time of delivery. The age of the child at fifth grade was calculated from the CARDIAC screening date and parent-reported child birthdate in the CARDIAC project. The sex of the child was recorded at birth and at fifth grade by all three projects. This study used the sex (male or female), recorded by the CARDIAC project in fifth grade. The race/ethnicity of the child was parent-reported in fifth grade. For our study we dichotomized the race as "white" and "other" based on the population distribution of WV (i.e., $94 \%$ white). ${ }^{90}$ Current literature shows inconsistent results of the association between maternal age and childhood CVD risk factors; ${ }^{91,92}$ thus maternal age at the time of infant's birth was included as a confounder and recorded by the WV Birth Certificate data. Maternal education has also been shown to be associated with CVD risk factors in childhood ${ }^{93}$ and also a predictor of maternal breastfeeding practices. ${ }^{94,95}$ Maternal education at time of birth was recorded as the number of years of education received ranging from 1 to 17 and was included as a continuous variable for the analysis. Current literature also shows that maternal socio-economic play an important role in the mother's decision and duration to breastfeed her child. ${ }^{94,96,97}$ Maternal health insurance at the time of delivery was assessed as a confounder variable (as a measure of socioeconomic factor) and categorized as a binary variable (Medicaid and non-Medicaid).

Family history of risk factors. The CARDIAC project collects information on family history of heart disease, coronary heart disease, heart attack, open-heart surgery, angioplasty, and death from heart disease; thus, we made a new variable called family history of CVD based on having family history of any one of these six variables. Family history of hypertension or high cholesterol is associated with having high blood pressure and abnormal lipid level in later life; $; 8,99$ the CARDIAC project collects information on family history of cholesterol (yes or no). 
Other infant and maternal characteristics. Additional covariates include number of previous pregnancies ( 0 or $\geq 1$ ), smoking during pregnancy (yes or no), weight gain during pregnancy (measured in pounds) and birth weight of the child (measured in grams). The literature shows that children from mothers with increasing parity (i.e., with one or more than one number of previous pregnancies; multiparous) have lower blood pressure, TC and LDL compared to children of mothers with no prior pregnancies (nulliparous). ${ }^{100,101}$ Maternal smoking during pregnancy has also been shown to be associated with childhood CVD risk factors, ${ }^{96,102}$ and maternal smoking is also associated with breastfeeding initiation and duration. ${ }^{103}$ Current literature shows inconsistent association between gestational weight gain and higher risk of childhood CVD risk factors. ${ }^{104,105}$ We had information on maternal gestational weight gain and assessed for its role as a covariate in our study. Birth weight of the child is also associated with childhood CVD risk factor. ${ }^{102}$ The information on birth weight was available as a continuous variable in grams from the WV Birth Certificate data.

\section{Statistical Analysis.}

All statistical analyses were conducted in SAS version 9.3 (SAS Institute, Cary NC). TG was $\log$ transformed in all analyses. Independent samples t-tests were used to compare the means of dependent variables for the two breastfeeding groups (reported history of breastfeeding and no reported history of breastfeeding) and the magnitude of this association was calculated using Cohen's d effect size (Cohen's d $=\frac{\mathrm{M} 1-\mathrm{M} 2}{\text { SD pooled }}$ where SD pooled $=\frac{\text { s.d.1 }+ \text { s.d.2 }}{2}$ ). Bivariate relationships between reported history of breastfeeding (binary: yes vs. no) and the CVD risk factors (continuous variables) were assessed using Spearman correlation.

Seven separate multiple-regression analyses were performed for the 7 continuous DVs (SBP, DBP, TC, LDL, HDL, non-HDL, and TG). All CVD outcomes were first regressed on reported history of breastfeeding (model 1) (main independent variable) and then in separate models BMI percentile was included to assess for its role as a mediator (model 2). For the outcomes where the association was significant between reported history of breastfeeding and CVD outcomes (independent of obesity), additional covariates were added in the model (model 3 ). The decision to include the additional covariates was based on a priori hypotheses, existing literature, and associations that were found significant with the outcome variable at the bivariate level using Pearson (for continuous variables) or Spearman (for categorical variables) correlations.

Ordinary Least Squares (OLS) regression was utilized, which is the most common type of multiple regression model where: $y=a+b x 1+b x 2+b x 3+b x 4 \ldots b x 15$, where ' $y$ ' is the criterion 
DV, ' $a$ ' is the constant, ' $b$ ' is the slope weight values, and $x 1$ through $x 15$ are the values for the predictors (IVs). The slope ' $b$ ' gives us the change in the predicted value of $Y$, on average, for each unit increase in X. Regression assumptions were assessed for all variables. The regression $\mathrm{F}$ statistic was used to determine the overall significance of the regression model and a corresponding $\mathrm{p}$-value of $\leq 0.05$ was used for statistical significant. The association of each independent variable to the outcome variable was also assessed for significance (alpha $\leq 0.05$ ) by examining the parameter estimates and their corresponding t-test values in addition to the $95 \%$ confidence intervals (CI) of the t-statistic. The covariates were dropped one at a time from the regression model if they were not significant (with highest $p$-value greater than $p>0.05$ ).

However, if the main independent variable (reported history of breastfeeding) was not significant it was retained in the model regardless of its significance.

We report both standardized and unstandardized regression coefficients in order to assist in interpretation of the findings. The squared multiple correlations, $\mathrm{R}^{2}$, and adjusted $\mathrm{R}^{2}$ were interpreted as the effect sizes of the regression model (magnitude of the association). The study also performed the regression analysis with and without the main predictor variable (i.e., reported history of breastfeeding) in order to calculate the amount of variance shared between reported history of breastfeeding and CVD risk factors by calculating the change in $\mathrm{R}^{2}$ for outcomes assessed in model 3 only. Furthermore, the interaction between age and sex was also assessed and dropped from the model if they were not significant.

For the maternal CVD risk factors (TC, LDL, HDL, non-HDL, and TG), we first assessed for the bivariate relationship between the binary categorical variable 'reported history of breastfeeding' and maternal CVD risk factors (continuous variables) using Spearman correlations. Independent samples t-tests were used to compare the means of dependent variables for the two breastfeeding groups, and the magnitude of this association was calculated using Cohen's d effect size for significant differences only. If the bivariate relationships were significant, then multiple regression analysis was planned to regress the outcome variable on the reported history of breastfeeding variable adjusting for additional covariates.

Sensitivity analyses. To explore whether there were sex differences we performed chisquare tests on breastfeeding groups, in addition to including the interaction term between sex and reported history of breastfeeding in the linear regression analysis. Many researchers believe that preterm and term infants are two separate populations with different rates and risks of morbidity and mortality in later life. ${ }^{106-110}$ The percentage of all infants who were reported to being breastfed was $43.03 \%$ in this population overall, and the frequency of reported history of breastfeeding was similar in full-term $(43.17 \%)$ and preterm $(41.75 \%)$ infants. Therefore, we 
performed all the analyses with children born both preterm and full-term combined to determine if there were any observed differences in the outcomes assessed. We also performed a sensitivity analysis on the missing data, and used multiple imputation techniques to address the missing data for the maternal lipid data. Lastly, we performed a post-hoc sensitivity analysis by adjusting for fasting levels in the regression analysis for children. There is a recent debate on whether to require prescreening fasting when assessing lipids in children in population based studies. Steiner and colleagues using data from NHANES 1999-2008 $(\mathrm{N}=12,744)$ found that the lipid and lipoprotein test results for fasting and non-fasting children were minor and do not have clinical relevance. They concluded that prescreening fasting should not be required for children in order to decrease the burden of childhood lipid screening. ${ }^{129}$ Nearly $20 \%$ of our study population in fifth grade was not fasting before screening.

\section{Results}

A total of 11,980 participants were available for analysis with the merged data. After excluding the infants born preterm (i.e. $<37$ weeks of gestation: $1,190(10.2 \%)$ ), the final number of participants for this study was 10,457. The study had lipid profiles on only 131 mothers, of which 127 were biological mothers in the complete dataset. For data restricted to full-term infants only, the study had lipid profile on 115 mothers, of which 112 were biological mothers.

From the CARDIAC project data, the mean age of the children in fifth grade was 10.97 $( \pm 0.47), 55 \%$ were female, $94 \%$ infants were white. The mean BMI percentile of fifth graders was $72.58 \%( \pm 28)$ and $43.2 \%$ of the parents reported breastfeeding the index child at birth. The CVD risk factor data was available for approximately $93 \%$ of children. However, the maternal lipid data was available for only $1 \%$ of the mothers. The detailed population characteristics of the children as well as the mothers' characteristic are available in Table 2.

Children who had a reported history of breastfeeding compared to children with no reported history of breastfeeding had significantly lower mean SBP, DBP, and TG and significantly higher for mean HDL in fifth grade. The mean difference was $-1.3 \mathrm{mmHg}(95 \% \mathrm{CI}$ : -1.97, -0.81), -0.81 mmHg (95\% CI: $-1.26,-0.33),-0.08 \mathrm{mg} / \mathrm{dL}$ (95\% CI: $-0.10,-0.05)$, and 0.9 $\mathrm{mg} / \mathrm{dL}(95 \% \mathrm{CI}: 0.33,1.56)$ for SBP, DBP, TG, and HDL cholesterol levels respectively (Table 3). The effect size calculated using the Cohen's d formula ranged from 0.07 to 0.16 for the significant outcomes (SBP, DBP, TG and HDL). The mean differences were not significant for TC, LDL, and non-HDL among children who were reported to be breastfed compared to children who were not breastfed. For the mothers, none of the mean lipid levels were significantly related 
to mothers who reported to have breastfed as compared to mothers who did not report breastfeeding the index child.

Consistent with the t-test results, the correlations between reported history of breastfeeding (yes vs. no) and SBP $(\mathrm{N}=6647, \mathrm{r}=-0.06, \mathrm{p}=0.0001)$, DBP $(\mathrm{N}=6648, \mathrm{r}=-0.04, \mathrm{p}=$ $0.0007)$ and $\mathrm{TG}(\mathrm{N}=6435, \mathrm{r}=-0.07, \mathrm{p}=0.0001)$ were significant and negative, whereas the correlation between reported history of breastfeeding (yes vs. no) and HDL was significant and positive $(\mathrm{N}=6647, \mathrm{r}=0.04, \mathrm{p}=0.0011)$ (Table 4$)$. The correlation between reported history of breastfeeding and child's TC, LDL, and non-HDL was not significant. Maternal lipid levels were not significantly correlated to reported history of breastfeeding (yes vs. no) (Table 4). However the direction of associations was consistent with the literature that suggests a protective association between breastfeeding and maternal lipid levels. The results demonstrated a positive correlation between mothers who reported to have breastfed vs. not breastfed and TC, LDL, nonHDL, and TG and a negative correlation between these two groups and maternal HDL levels.

The unadjusted regression analysis showed that children who had a reported history of breastfeeding compared to children with no reported history of breastfeeding had significantly lower SBP ( $b=-1.39 \mathrm{mmHg}$; 95\% CI: $-1.97,-0.81 ; \mathrm{p}<0.0001)$, DBP ( $b=-0.79 \mathrm{mmHg} ; 95 \% \mathrm{CI}$ : $-1.26,-0.33 ; \mathrm{p}=0.0009), \log -\mathrm{TG}(\mathrm{b}=-0.08$ (95\% CI: $-0.1,-0.05 ; \mathrm{p}<0.0001)$, and higher HDL $(\mathrm{b}$ $=0.95 \mathrm{mg} / \mathrm{dL}(95 \%$ CI: 0.33, 1.56; $<<0.0001)$ (Table 5, model 1). However, adjustments for the child's current BMI decreased the associations of reported history of breastfeeding to SBP $(b=-$ $0.77 \mathrm{mmHg} ; 95 \% \mathrm{CI}:-1.32,-0.23 ; \mathrm{p}=0.005)$ and $\log -\mathrm{TG}(\mathrm{b}=-0.05 ; 95 \% \mathrm{CI}:-0.07,-0.03 ; \mathrm{p}=$ $0.048)$, but eliminated the association to DBP $(b=-0.44 \mathrm{mmHg} ; 95 \% \mathrm{CI}:-0.89,0.02 ; \mathrm{p}=0.06)$ and HDL $(b=0.28 \mathrm{mg} / \mathrm{dL}(95 \% \mathrm{CI}:-0.29,0.86 ; \mathrm{p}=0.335)$ (Table 5, model 2). Adjustments for additional socio-demographic variables eliminated the association between reported history of breastfeeding and SBP $(b=-0.43 \mathrm{mmHg} ; 95 \% \mathrm{CI}:-0.98,-0.13 ; \mathrm{p}=0.1349)$ and attenuated the association with $\log$-TG but remained significant $(b=-0.04 ; 95 \% \mathrm{CI}:-0.06,-0.01 ; \mathrm{p}=0.008)$ (Table 5, model 3).

The results of the multiple regression analysis showed that reported history of breastfeeding was significantly and inversely associated with TG of children with inclusion of covariates in the model $\left(\mathrm{F}(8,5339)=118.84 ; \mathrm{p}<0.0001\right.$; adjusted $\left.\mathrm{R}^{2}=0.1499\right)$. Covariates including BMI percentile in fifth grade, child's age, sex, race, maternal education at birth, number of previous pregnancies, and family history of cholesterol were statistically significant in predicting variance in TG of the child at 11 years of age (Table 6). The regression model showed that among children who had a reported history of breastfeeding there was a $0.04(b=-0.04 ; 95 \%$ CI: $-0.06,-0.01, \mathrm{t}=-2.65, \mathrm{p}=0.008)$ decrease in the log-transformed TG levels compared to 
children who did not have a reported history of breastfeeding. The amount of variance shared between reported history of breastfeeding and childhood TG levels was $0.13 \%$ (change in $\mathrm{R}^{2}$ ).

No sex differences were observed in the additional (sensitivity) analysis that examined the interaction of reported history of breastfeeding by sex on blood pressure and lipid levels of children ( $b=-0.03 ; 95 \%$ CI: $-0.08,0.02, t=-1.15, p=0.2495)$. The results of the sensitivity analyses that included all infants (preterm and full term) showed similar findings for both the children and the mothers' data. For example, the regression results for children born both preterm and full-term combined showed that reported history of breastfeeding was significantly and inversely associated with log-TG of children in fifth grade $(-0.03 ; 95 \% \mathrm{CI}:-0.0513,-0.0005, \mathrm{p}=$ $0.046)$ with inclusion of covariates in the model $\left(\mathrm{F}(8,6146)=139.58 ; \mathrm{p}<0.0001\right.$; adjusted $\mathrm{R}^{2}=$ 0.1527). After multiple imputation for the missing data for the mothers, none of the lipid levels were significantly correlated with mothers' reported history of breastfeeding (mothers who reported to have breastfed compared to mothers who did not) (supplemental files tables S1-5). We performed a sensitivity analysis (post-hoc) and adjusted for the child's pre-screening fasting status. The regression results showed similar findings when adjusted for pre-screening fasting status vs. not adjusting for fasting status [for e.g. the beta coefficient for the association between breastfeeding and log-TGs adjusting for fasting was -0.035 (95\% CI: $-0.06,-0.01 ; p=0.0113)$ compared to -0.036 without adjusting for fasting status $(95 \% \mathrm{CI}:-0.06,-0.01 ; \mathrm{p}=0.008)]$.

\section{Discussion}

Overall, the results showed that children who had a reported history of breastfeeding (yes vs. no) had significantly lower SBP, DBP, TG, and higher HDL at the bivariate level (based on the results of the t-test, Spearman correlation and unadjusted linear regression results). When adjusted for the child's current BMI percentile in fifth grade, the association attenuated for SBP and TG but remained significantly and positively associated with reported history of breastfeeding. Thus, results suggest that BMI is a potential mediator for the association between reported history of breastfeeding and DBP and HDL levels. However, adjustments for additional covariates eliminated the association of reported history of breastfeeding to SBP but remained significant for TG levels.

\section{Blood Pressure in Children}

Our results are consistent with studies that found no differences in the mean SBP and DBP of children who were breastfed as compared to those not breastfed during infancy. ${ }^{29-36}$ 
However, several studies have found the opposite ${ }^{23-26}$ including two earlier systematic reviews with meta-analysis. ${ }^{27,28}$ These systematic reviews concluded that the pooled mean difference in SBP (but not DBP) was significantly lower among infants who were breastfed, ${ }^{27}$ while the later systematic review with meta-analysis found significant reduction in both SBP and DBP. ${ }^{28}$ Systematic reviews with meta-analysis occupy the highest levels of evidence in the hierarchy of study designs. ${ }^{111}$ The Cochrane Collaboration, an organization dedicated to the conduct of systematic reviews in healthcare, recommends that reviews on a given topic be updated every two years. ${ }^{112,113}$ Our results on both SBP and DBP are consistent with the evidence provided by the most recent systematic review with meta-analysis published in $2015 .{ }^{37}$ Horta and colleagues demonstrated based on 10 studies for age groups 10-19 that SBP was lower but not significant among those subjects who had been breastfed [mean difference: $-1.03 \mathrm{mmHg}(95 \% \mathrm{CI}:-2.07$; 0.02)], whereas no association was observed for DBP based on 8 studies for age groups 10-19 [mean difference: $-0.1 \mathrm{mmHg}(95 \% \mathrm{CI}:-0.65 ; 0.45)$ ]. Furthermore, this meta-analysis showed that the mean difference was inversely related to study size. Studies with more than 1000 participants included the null value. Our study further demonstrated that the association between reported history of breastfeeding and childhood blood pressure (SBP and DBP) is not independent of child's current BMI and socio-demographic variables, thus suggesting the role of child's current BMI as a potential mediator. Breastfeeding has shown to be protective for childhood obesity as demonstrated by several studies ${ }^{18-21,37,114}$ including our earlier work with WV fifth grade children. ${ }^{115}$

\section{Lipid in Children}

The results of the current study are also consistent with studies that found no association between TC, ${ }^{37,42,43}$ LDL, ${ }^{43}$ and non-HDL levels among breastfed vs. non-breastfed children. The results of two systematic reviews with meta-analysis using random effects model showed that the mean TC and LDL levels in children were not significantly different comparing breastfed infants to non-breastfed infants [(mean difference TC $=0.00 \mathrm{mmol} / \mathrm{L} ; 95 \% \mathrm{CI}:-0.07,0.07)$ and (mean difference $\mathrm{LDL}=0.01 \mathrm{mmol} / \mathrm{L} ; 95 \% \mathrm{CI}:-0.07,0.08)] .{ }^{43}$ The most recent meta-analysis also found no significant difference in the mean TC levels for children ages 10-19 between breastfed and non-breastfed infants (mean difference $\mathrm{TC}=0.01 \mathrm{mmol} / \mathrm{L} ; 95 \% \mathrm{CI}:-0.06,0.08) .{ }^{37}$

Of greater interest to us were the relationships that proved significant: in particular, HDL and TG. For HDL our study found a significant positive association among fifth grade children who were reported to have been breastfed during infancy, with a mean difference of $0.9 \mathrm{mg} / \mathrm{dL}$ (95\% CI: 0.33, 1.56). However, the association attenuated after adjustment for the child's current 
BMI and became non-significant. The non-significant results are consistent with other studies ${ }^{42,44}$ that also adjusted for the child's current BMI. ${ }^{42}$ This finding also suggests that BMI is a potential mediator for the association between breastfeeding and HDL levels in this population.

We found a significant association between log-transformed TG levels among fifth grade children who had a positive history of breastfeeding independent of the child's BMI, sociodemographic and lifestyle characteristics. To our knowledge very few studies have examined this association for childhood TG levels. One study found a significant negative association in 17 years old boys but not among girls ${ }^{116}$ and another study among 18-year-old boys did not find any difference in never vs. ever breastfed adolescents. ${ }^{44}$ Yet, another population based study found that among 6 year old children this association was not significant after adjusting for age, sex, family-based socio-demographic, maternal lifestyle-related and childhood factors. ${ }^{117} \mathrm{We}$ did not find any differences by gender for the association between breastfeeding and TG levels. The mean difference was significantly lower among children who had a reported history of breastfeeding (yes vs. no) during infancy -0.08 (95\% CI: -0.10, -0.05), and the correlation was small $(r=-0.07, p<0.0001)$ but also significant. The standardized regression coefficient of the unadjusted association was -0.07 , which attenuated but remained significant after adjusting for childhood BMI percentile (-0.05) and remained significant after adjusting for additional covariates as well (-0.03) (covariates included BMI percentile, child's age, sex, race, maternal education at birth, number of previous pregnancies, family history of cholesterol). Based on these results, we hypothesize that this association is potentially partially mediated by the child's current BMI levels. However, the change in $\mathrm{R}^{2}$ showed that reported history of breastfeeding accounted for less than $0.2 \%$ of unique variance in TG levels of fifth grade children, suggesting a small effect size.

Interpreting significant covariates included in the study may be of some interest (Table 5). The results suggest that boys have lower TG levels compared to girls in fifth grade. Some studies have noted that boys have lower TG levels compared to girls at ages $10-14^{118}$ and other studies have found that TG levels in girls and boys are generally similar during early childhood, but in adolescence girls have lower TG levels than boys. ${ }^{119,120}$ These studies also noted that TG values increase sequentially with the increase in pubertal stage. Although this study did not have information on pubertal status we can hypothesize that perhaps more girls had reached puberty in fifth grade compared to boys in this cohort, explaining the higher TG levels in girls in fifth grade WV children. Although the age range in our study was only 5 years (9.3 to 14.14), we observed that there was a significant increase in the level of TGs with age as observed in other studies as well. ${ }^{121}$ The results also showed that white children had higher TG levels compared to other racial 
groups, which is also consistent in the literature as well. ${ }^{121,122}$ Although most of the previous research compared whites to blacks, this study collapsed the minority racial groups in one group based on the population distribution of WV as well as our study (i.e., 94\% white). ${ }^{90}$ Our study demonstrates that increasing years of maternal education at time of the child's birth was associated with lower child's TG levels. Although previous studies examining this association (between maternal education and child's TG level) have shown inconsistent results. ${ }^{123,124}$ Our results also demonstrated that multiparous mothers at birth of the index child had children with lower TG levels compared to nulliparous mothers, which is also consistent with the literature. ${ }^{100}$ Lastly, children who had a family history of hypercholesterolemia had significantly higher TG levels in fifth grade WV children, a finding echoed by previous research as well. ${ }^{125}$

As mentioned earlier that there were significant differences in TG level by gender but no significant interaction between gender and reported history of breastfeeding was observed. Furthermore the results of the sensitively analysis performed on the complete data that included all infants found similar results to the data restricted to full term birth infants only.

\section{Lipid in Mothers}

The results of our study found no significant correlation or significant mean difference in any of the lipid levels of mothers who reported to have breastfed as compared to mothers who did not breastfeed. Although the results were not significant it is important to note that the direction for the association was positive for TC, LDL, non-HDL, and TG and negative for HDL. To our knowledge very few studies have examined this association. Our results contrast with few studies that have found a protective association between breastfeeding and maternal lipid profile. ${ }^{11,48,53}$ However, our results are in concordance with the results of a prospective cohort study that did not find an association between breastfeeding duration and any of the serum lipid markers (TC, LDL, HDL, and TG) at 3 years postpartum. ${ }^{56}$ Several factors may explain our disparate results. The earlier studies examined lifetime lactation duration of at least $3 \mathrm{month}^{54}$ or lifetime duration of lactation of more than 1 or 2 years to demonstrate this protective association. ${ }^{11,48}$ Our study did not have information on lactation duration or lifetime duration of lactation, which is the basis of most previous research. Since our study asked mothers if they breastfed or not, it is possible that most mothers who initiated breastfeeding, failed to continue to breastfeed for longer durations. Data from the National Immunization Survey 2011 show that 59\% of the mothers ever breastfed their infant while only $12.2 \%$ of the mothers in WV exclusively breastfeed for the first six months and nearly $16 \%$ breastfed for up to one year. ${ }^{13}$ Additionally, only a small percent of mothers of participating CARDIAC students took advantage of the free vouchers for lipid testing, resulting 
in very low participation rates among this particular sample. Furthermore, experts in the field also suggest that no existing study has collected comprehensive data to explain the apparent association between lifetime lactation and maternal metabolic risk adjusting for several confounding factors. ${ }^{70}$ It has been noted in the U.S. as well as in WV that mothers who breastfeed belong to higher SES and engage in healthy behaviors. ${ }^{126,127}$ Thus future studies need to collect wide ranged longitudinal data to examine this association in mothers accounting for several socio-demographics, genetic and lifestyle factors.

\section{Limitations of the Study}

Some of the limitations of the study include lack of information on possible important confounder variables such as parental adiposity status, maternal pre-pregnancy weight, rapid weight gain during the first year of life, pubertal status of the child, family history of hypertension, physical activity, and dietary behaviors. Furthermore, the CARDIAC project only collects parental lipid data and thus the maternal CVD risk factors were limited to lipid profile only. Additionally, maternal lipid data was available for a very small sample of the population as few mothers took advantage of the free vouchers for lipid testing, thus limiting the generalizability of the maternal sample population. Moreover, information on history of breastfeeding was obtained retrospectively and thus is subject to recall bias and also to social desirability bias. The measure also did not inquire about the method, extent, or the lifetime duration of lactation. Lastly, due to the unique population characteristics of this state, the results may not be generalizable beyond the participants included in this study.

\section{Strengths of the Study}

Despite these limitations, this study afforded the strengths of examining the association between reported history of breastfeeding and CVD health outcomes in a large dataset, longitudinally, using linked data from two cross-sectional studies and adjusting for numerous important covariates. Although all the associations attenuated after adjustment for the child's BMI, the association remained significant for TG levels independent of the child's current BMI, socio-demographic, genetic and other lifestyle factors. In addition, the results from the regression analysis showed that although the predictors accounted for nearly $15 \%$ of the variance in TG levels, reported history of breastfeeding uniquely accounted for less than $1 \%$ of the variance in TG levels. Although this effect size is small, it is a novel finding in 11-year-old children. Earlier studies included 6-year-old children or older adolescents or young adults ranging from ages 1725. ${ }^{116} 44,117,128$ Lastly, for the maternal CVD risk factors; the current study demonstrates some 
important preliminary results (i.e., correlations in the correct direction for lipid levels and breastfeeding). Thus, providing an essential contribution for expanding the research examining the associations between breastfeeding as a protective perinatal factor and maternal cardiovascular health in subsequent years.

\section{Conclusion}

Breastfeeding has been shown to have numerous health benefits for both the mother and the child. Our earlier work has shown that breastfeeding is protective for childhood obesity. This study further adds that breastfeeding is protective for child's TG levels at 11 years of age after accounting for several demographic and maternal characteristics, thus adding to the argument of promoting breastfeeding as a preventive measure against future CVD. The observed effects of breastfeeding as an early-life determinants or a distal protective factor for the child's TG lipid level was small. However, these small changes can impact the burden of CVD risk factors prevalence in rural Appalachian state of WV. The fact that we were not able to show the protective effect on blood pressure and other child or maternal lipid levels does not mean that breastfeeding promotion should be ignored. Future large prospective studies are needed to assess and understand the association of breastfeeding with both maternal and child CVD risks factors at various life stages. 


\title{
Chapter 4: Childhood obesity and adult cardiovascular disease risk factors: a systematic review with meta-analysis.
}

\begin{abstract}
Introduction: Previous systematic reviews have led to conflicting findings regarding the relationship between childhood obesity and adult cardiovascular disease risk factors. The purpose of this study was to use the aggregate data meta-analytic approach to address this gap.

Methods: Studies were included if they met the following criteria: (1) longitudinal and cohort studies (including case-cohort), (2) childhood exposure and adult outcomes collected on the same individual over time, (3) childhood obesity, as defined by the authors, (4) Englishlanguage articles, (5) studies published up to June, 2015, (6) one or more of the following CVD risk factors [systolic blood pressure (SBP), diastolic blood pressure (DBP), total cholesterol (TC), high-density lipoprotein cholesterol (HDL), low-density lipoprotein cholesterol (LDL), non-highdensity lipoprotein cholesterol (non-HDL), and triglycerides (TG)] (7) outcome(s) not selfreported, and (8) exposure measurements (child's adiposity) assessed by health professionals, trained investigators, or self-reported. The search strategy included (1) electronic searches in multiple databases (PubMed (MEDLINE), Web of Science, and Scopus) on June 5, 2015, and (2) cross-referencing from the reference lists of all retrieved articles (citation tracking). Fisher's $\mathrm{r}$ to $z$ score was calculated for each study for each outcome. Pooled effect sizes were calculated using random-effects models while risk of bias was assessed using the STROBE instrument. Heterogeneity was assessed based on fixed-effect models. In order to try and identify sources of heterogeneity, random-effects meta-regression was also performed.

Results: Of the 4875 citations reviewed, a total of 23 studies were included in the systematic review and 21 in the meta-analysis. The findings suggest that childhood obesity is significantly and positively associated with adult SBP $(\mathrm{Zr}=0.11 ; 95 \% \mathrm{CI}: 0.07,0.14)$, DBP ( $\mathrm{Zr}=$ 0.11 ; 95\% CI: $0.07,0.14)$, and TG ( $\mathrm{Zr}=0.08 ; 95 \% \mathrm{CI}: 0.03,0.13)$, and significantly and inversely associated with adult HDL ( $\mathrm{Zr}=-0.06$; 95\% CI: $-0.10,-0.02)$. For 3-6 studies that adjusted for adult body mass index (BMI), associations were reversed for all outcomes, suggesting that adult BMI may be a potential mediator. Nine studies had more than $33 \%$ of items that placed them at an increased risk for bias.

Conclusions: Childhood obesity is a risk factor for adult SBP, DBP, HDL, and TG. Well-designed, longitudinal studies with high quality of reporting as well as data on both unadjusted and adjusted (for adult adiposity) associations are needed before any definitive conclusions can be reached
\end{abstract}




\section{Introduction}

\section{Overview}

Overweight and obesity during childhood and adolescence is a major public health problem. One of the immediate health implications of childhood and adolescent obesity includes the development of cardiovascular disease (CVD) risk factors during childhood and adolescence..$^{1-4}$ According to the World Health Organization, CVD mortality increased globally from 14.4 million in 1990 to 17.5 million in 2005 and is projected to rise to more than 23.6 million by $2030 .^{5-8}$ However, in the United States (U.S.), CVD mortality has been decreasing from $34.3 \%$ deaths in 2006 to $30.8 \%$ of all deaths in $2013 .{ }^{5,9}$ Nevertheless, it is still the leading cause of death. ${ }^{10}$ According to the 2016 Heart Disease and Stroke Statistics update, more than one third of U.S. adults have at least one type of CVD, ${ }^{5}$ which is also projected to rise to more than $40 \%$ by $2030 .^{5,11}$ Several well-established adult CVD risk factors have been found during childhood. These include, but are not necessarily limited to, high blood pressure (BP), poor lipid profile, impaired glucose tolerance, and metabolic syndrome. ${ }^{12-14}$ Importantly, data shows that these risk factors are amplified in the presence of pediatric obesity, referred to by Ford et al. as 'obesity-associated risk factors for CVD.' ${ }^{13,14}$ Most notably, a population-based study estimated that $70 \%$ of obese children and adolescents between the ages of 5 to 17 have at least one risk factor for CVD. ${ }^{1}$

Despite the high prevalence of both childhood and adolescence obesity and adult CVD, studies examining the relationship between childhood obesity and adult CVD have yielded conflicting results. ${ }^{13,15-18}$ However, adult adiposity is an established risk factor for developing adult $\mathrm{CVD}^{19,20}$ and there is much evidence to suggest that overweight adolescents have a $40 \%$ $80 \%$ chance of becoming overweight or obese adults. ${ }^{21-23}$ Thus, it remains unclear whether childhood obesity is an independent risk factor for adult CVD risk factors or whether childhood obesity persists as adult obesity and indirectly increases the risk of adult CVD. ${ }^{24,25}$

\section{Obesity and CVD Risk Factors}

High BP and poor lipid profile are independent risk factors for CVD. However, pediatric obesity is also an independent risk factor for high BP and poor serum lipid and lipoprotein concentrations (higher total cholesterol (TC), triglycerides (TG), low-density lipoprotein cholesterol (LDL) and lower high-density lipoprotein cholesterol (HDL) concentrations) during childhood and adolescence. ${ }^{26-31}$ Children and adolescents with less than optimal BP or cholesterol levels are more likely to develop high BP and less than optimal cholesterol levels during 
adulthood compared to children/adolescents with a normal BP and lipid profile ${ }^{32-34}$. Moreover, abnormal serum lipoprotein concentrations due to pediatric obesity and its correlation with poor lipid profile during adulthood also remain a controversial topic in the literature ${ }^{3,35}$. Data also shows that childhood CVD risk factors may persist into adulthood, ${ }^{25}$ however, the mechanism from transition from CVD risk factors from childhood to adulthood is still unclear. ${ }^{36}$ Recent systematic reviews suggest that the relationship between childhood obesity and adult high BP or poor lipid profile is weak, possibly because the results are confounded by adult obesity. ${ }^{18,37}$ Given that childhood obesity as an independent risk factor for CVD in adults is not well established, we aim to investigate the association between childhood obesity and select adult CVD risk factors, i.e., BP, lipids, and lipoproteins.

\section{Critical Evaluation of Existing Knowledge}

When proposing to conduct a systematic review (SR) with meta-analysis (MA) on a topic, it is important to examine previous SRs and MA on this topic. To the best of the investigative team's knowledge, four SRs and one MA have been conducted on the relationship between childhood obesity and adult CVD risk factors. ${ }^{18,37-40} \mathrm{~A}$ brief description of these studies is shown in Table 1. The SRs and MA found were based on a systematic literature search in PubMed on February 2, 2015, using the following search strategy: ((obes* OR overweight) AND (child* OR adolesc*) AND (systematic review OR meta-analy*) AND (blood pressure OR cholesterol OR lipids OR lipoproteins OR cardiovascular) AND adult*). The search strategy can found in Additional file 1.

To the best of our knowledge, we are not aware of any meta-analytic research that utilized a systematic way for critically appraising the association between childhood obesity and adult CVD risk factors based on current guidelines. ${ }^{41}$ As can be seen in Table 1, four SRs published on this topic from 2010-2012 provided qualitative evidence but did not show any quantitative evidence on the association between childhood obesity and adult CVD risk factors (BP and lipid profile). While one MA was conducted four years ago on this topic, it was limited to a select four cohorts only, ${ }^{40}$ thereby possibly biasing results. Moreover, this MA did not calculate the association between childhood obesity and TC as well as between childhood obesity and adult non-high-density lipoprotein cholesterol (non-HDL) levels. This is important since nonHDL (non-HDL = TC minus HDL) has been shown to be better marker of risk for coronary artery disease and stroke compared to LDL. ${ }^{42,43}$

Of the four SRs listed in Table 1, two included hypertension as one of the main outcomes ${ }^{37,38,44}$ while the other reported results for resting SBP and DBP. ${ }^{37}$ Another study focused 
on the lipid profile as the main outcome of interest. ${ }^{18}$ With respect to years covered for those studies that included hypertension, Park et al, included studies published between 1980-2011, ${ }^{38}$ Reilly et al. included studies from January 2002 to mid-June 2010, ${ }^{39}$ while Lloyd et al, searched online electronic databases, i.e., PubMed (MEDLINE) and ISI Web of Science from their inception up to July 2008 for the SR with hypertension as the outcome, ${ }^{37}$ and up to July 2010 for the SR with serum cholesterol levels as the outcome. ${ }^{18}$ The Cochrane Collaboration, an organization dedicated to the conduct of SRs in healthcare, recommends that SRs on a given topic be updated every two years. ${ }^{45,46}$ Based on this recommendation, the last SR was published in 2012, thus suggesting that this work needs to be updated. In addition, all previous SRs included data where adiposity was measured using BMI for both children and adults. ${ }^{18,37-40}$ However, research has shown that BMI is not an ideal marker for adiposity ${ }^{47,48}$ and including other definitions or classifications of adiposity may help in identifying other potentially eligible studies that have looked at this association. Finally, the methodological quality of these previous systematic reviews could have been better. ${ }^{18,37-40}$ Using the Assessment of Multiple Systematic Reviews (AMSTAR) Instrument, ${ }^{49}$ we evaluated the methodological quality of the previously described SRs. Item-by-item results for these studies are shown in Table 2. As can be seen, the overall score for each SR ranged from $40 \%$ to $80 \%$ while scores for each question ranged from $0 \%$ to $100 \%$. The questions with the three lowest scores included: (1) status of publication, (2) including a list of both published and unpublished studies, and (3) assessment for the likelihood of publication bias. These findings provide further support for an updated, high-quality SR with MA on the relationship between childhood obesity and selected adult CVD risk factors, i.e., BP, lipids, and lipoproteins.

\section{Significance of the Topic}

SRs with MA occupy the highest levels of evidence in the hierarchy of study designs. ${ }^{50}$ This structured and standardized approach has been used to make health care decisions and inform policy makers by analyzing prior findings as well as summarizing, synthesizing and critically appraising evidence on a specific topic in the literature. ${ }^{51}$ While several SRs, ${ }^{18,37-39}$ and one $\mathrm{MA},{ }^{40}$ have examined the association between childhood obesity and adult CVD, the investigative team is not aware of any current and thorough SR with MA on this topic. Thus, the specific aim of this study was to conduct a SR and MA to critically evaluate the available evidence regarding the relationship between childhood obesity and selected adult CVD risk factors. The results of this study can contribute to the planning and implementation of preventive strategies for promoting cardiovascular health during the various life stages as well as provide 
direction for the future conduct and reporting of research on this topic.

\section{Research Design and Methods}

\section{Purpose}

The purpose of this study was to conduct a SR and MA of studies that have examined the association between childhood obesity and the following adult CVD risk factors: (1) resting systolic blood pressure (SBP), (2) resting diastolic blood pressure (DBP), (3) TC, (4) HDL, (5) LDL, (6) non-HDL and (7) TG. A secondary aim of the study was to examine whether this association persists after adjusting for adult obesity.

We conducted a SR with MA by following the Cochrane Collaboration's recommendations and guidelines for conducting SRs and MA for observational studies, ${ }^{45,52}$ as well as the Preferred Reporting Items for Systematic reviews and Meta-Analyses (PRISMA) ${ }^{53,54}$ statement. In addition, we registered this study in PROSPERO, an international registry for SRs (Protocol number: PROSPERO 2015:CRD42015019763). ${ }^{55}$

\section{Study Eligibility}

The eligibility criteria for the studies included or excluded in the MA need to be well defined and established $a$ priori. ${ }^{56}$ The Cochrane acronym $\operatorname{PICO}(\mathrm{S})$ (population, intervention, comparison, outcomes, study design or study setting), was utilized to ensure that all key components are covered prior to starting the review process. The eligibility criteria for the study included: (1) longitudinal and cohort studies (including case-cohort), (2) childhood exposure and adult outcomes collected on the same individual over time, (3) main exposure variable of the child's overweight and obesity status (BMI age-and sex-specific percentiles, percent body fat, fat mass, waist circumference (WC), waist-to-hip ratio, visceral adipose tissue, skin fold thickness, body weight, BMI z-score, BMI or other measures used to assess overweight and obesity in populations), ${ }^{57}$ (4) studies available in English-language, (5) studies published up to June, 2015, (6) one or more of the following CVD risk factors as the primary outcome measure: (SBP, DBP, TC, LDL, HDL, non-HDL, and TG), (7) outcome measurements taken by health professionals or trained investigators but not based on self-report data, (8) exposure measurements (child's adiposity) assessed by health professionals, trained investigators, or self-reported. Exclusion criteria included the following: (1) review articles, (2) cross-sectional study designs, (3) casecontrol study designs, (4) case reports, (5) comments, (6) letters, (7) animal studies, (8) studies published in non-English language sources, (9) presentations from conference meetings, (10) 
unpublished studies (abstracts, master theses, dissertations, etc.), (11) studies in which the outcome(s) were self-reported and, (12) studies outside general population.

We utilized a number of various sources and methods to identify the maximum number of eligible articles. These included electronic database searches as well citation tracking from the retrieved articles. We also assessed for publication bias, which occurs when the published literature is systematically unrepresentative of the population of all completed studies ${ }^{58,59}$ Grey literature refers to unpublished reports, conference abstracts, thesis and dissertations, articles in obscure journals, reports, rejected or un-submitted manuscripts. ${ }^{60}$ Due to limited resources, we did not search the grey literature but utilized numerous sources to identify the published literature. While MA that exclude grey literature may tend to overestimate ESs, ${ }^{60-62}$ some researchers have argued that the methodological quality of grey literature is low compared to published literature due to a lack of formal quality control and peer review, ${ }^{63,64}$ and thus, can jeopardize the quality of MA given that the quality of MA is dependent on the quality of the studies included in the analysis. ${ }^{65}$ Alternatively, others have argued that there is limited evidence that grey literature is of lower quality than published trials. ${ }^{62}$ However, as previously mentioned, we excluded grey literature because of limited resources as well as the difficulty to access this type of literature, the latter of which can also potentially bias results.

In order to control for multiple publication bias (multiple publications from the same population), only one set of data that included the greatest amount of relevant information on the participants was included. Given the lack of resources, we also excluded studies that were not published in the English language. Meta-analyses that restrict studies by language can potentially overestimate the outcome effects by only $2 \%$, and the percentage of non-English studies traditionally included in meta-analyses is very small. ${ }^{66}$

\section{Data Sources}

A SR provides a comprehensive search for all potentially relevant literature for a specific research question. ${ }^{67}$ For the current study, we searched multiple electronic databases and crossreferenced from the bibliographies of all retrieved articles (citation tracking). An information retrieval specialist (Health Sciences librarian, JS) assisted in the planning of the literature search and in identifying and creating correct Boolean operators and search strings for the different electronic database searches. ${ }^{68}$ All aspects of the searches were documented with respect to name of the data source, journal, date of the search, person responsible etc. For this study, we searched the following electronic databases on June 5, 2015: (1) PubMed (MEDLINE), (2) Web of Science, and (3) Scopus. We first conducted a preliminary search in PubMed and searched 
forward from the date of the first study that met our search criteria (1975). The PubMed search string used was as follows: "(obesity OR obese OR overweight OR fat OR adipos* OR "body mass index" OR BMI) AND (child* or adolesc*) AND ("blood pressure" OR hypertension OR cholesterol OR lipid OR lipids OR lipoprotein OR lipoproteins OR cardiovascular) AND (observational OR cohort OR longitudinal) AND adult* AND (human OR humans)." Each search was conducted separately and then downloaded as a separate file using Endnote X7. ${ }^{69}$ All duplicates were removed electronically and then manually.

\section{Study Selection}

In order to minimize selection bias, two researchers (AU and CL) independently screened studies for eligibility by reviewing the titles and abstracts of articles based on the pre-defined eligibility criteria (see previous section on study eligibility). If the inclusion or exclusion criteria could not be decided based on the title and abstract, full-text articles were retrieved and the decision was made accordingly. After independent study selection was performed, the two reviewers met and reviewed every selection for agreement. Cohen's kappa statistic ${ }^{70}$ was used to measure inter-selection agreement and discrepancies were resolved by consensus. If a decision could not be achieved, a content area and MA expert (GK) resolved any disagreement(s). Using Cohen's kappa statistic, the overall agreement rate prior to correcting discrepancies was 0.75 .

\section{Data Abstraction.}

Prior to data abstraction, a detailed codebook that could hold up to 200 items per study was developed by the research team in Microsoft Excel software (version 2011). ${ }^{71}$ The codebook included continuous variables, categorical variables, and free text information. The codebook developed was pilot-tested and revised by the investigative team. In order to avoid data abstraction bias, two authors (AU and CL) coded or extracted data from each selected article independently. The researchers then compared every data point for accuracy and consistency until $100 \%$ agreement was reached. If agreement could not be reached, a content area and MA expert (GK) resolved any disagreement(s).

\section{Risk of Bias Assessment}

The term 'risk of bias' is used to describe "a systematic error or deviation from the truth, in results or inferences." ${ }^{45}$ The Cochrane Collaboration emphasizes that risk of bias assessment should clearly differentiate between the quality of reporting and the quality of the underlying conduct of the research. ${ }^{45}$ Biases similar to randomized controlled trials (RCT) may also be 
present in a non-randomized study (NRS). These include selection bias, attrition bias, performance bias, detection bias, outcome-reporting bias, publication bias and dissemination bias. However, the nature and variety of NRS makes it more difficult for the groups to be comparable. Several of the important biases in longitudinal studies include (1) selective dropout bias, (2) participation bias, (3) selection bias (sampling frame, recruitment, consent rates, retention, loss to follow-up, item non-response), (4) interviewer bias, (5) response bias, (6) social desirability bias, (7) measurement bias, and (8) the role of potential confounders and residual confounding in explaining the findings from NRS. Numerous tools that have evaluated study quality or risk of bias of observational studies ${ }^{72}$ i.e., Downs and Black, ${ }^{73}$ Reisch, ${ }^{74}$ and Zaza ${ }^{75}$ still require modifications as there is no gold standard for assessing the risk of bias of observational studies. ${ }^{72,76}$ However, one tool, Strengthening the Reporting of Observational Studies in Epidemiology (STROBE), has been cited by several SR and MA for assessing the reporting of observational studies. ${ }^{77}$ The STROBE instrument consists of a checklist of 22 items that provides guidance on the reporting of observational studies to facilitate critical assessment and interpretation of results. ${ }^{78}$ For this study, we used the STROBE instrument to assess the quality of reporting of the included observational studies. This checklist facilitates in assessing the risk of potential bias in the title and abstract, introduction, methods, results, and discussion sections of articles. Each item was classified as "yes" (low risk), "no" (high risk), or "unclear". Two researchers (AU and CL) conducted all assessments independent of each other and examined the results at the study level as well as for each item. They then compared their selections for accuracy and consistency. Inter-rater agreement was assessed using Cohen's kappa statistic. Any disagreements were discussed and resolved until 100\% agreement was reached. Using Cohen's kappa statistic, the overall agreement rate prior to correcting discrepancies ranged from 0.70 to $0.94($ mean $=0.89, \mathrm{SD}=0.03)$.

\section{Statistical Analysis}

Descriptive statistics. Using Microsoft Excel $2011^{71}$ and after data cleaning, descriptive statistics were generated for continuous [sample sizes, means, medians, standard deviations, standard errors, 95\% confidence intervals (CI)] and categorical (frequencies and percentages) variables. Seven separate Microsoft excel sheets were generated for all seven outcomes [(1) SBP, (2) DBP, (3) TC, (4) HDL, (5) LDL, (6) non-HDL, and (7) TG)]. Any outcome with only one study was excluded from the meta-analysis. Each outcome was further analyzed separately if it was adjusted for adult adiposity. 
Calculation of effect sizes from each study. The effect size (ES) is the strength of an association between two variables. The two previous SRs by Lloyd et al. presented the correlation statistics for the individual studies, ${ }^{18,37}$ whereas Reilly et al. ${ }^{39}$ and Park et al. ${ }^{38}$ presented odds ratios (OR). However, the one MA on this topic used risk ratios (RR), also known as relative risks, as their ES measure. ${ }^{40}$ The a priori plan was to use RR as our ES in order to examine the association between childhood obesity and selected adult CVD risk factors (SBP, DBP, TC, HDL, LDL, non-HDL, and TG). However, because most studies reported a correlation between two continuous variables, a post hoc decision was made to use correlation statistics (Fishers $\mathrm{r}$ to $\mathrm{z}$ score) instead of RR to serve as the main ES index. ${ }^{79}$ All other ESs (OR, mean differences) were converted to correlation statistics using Comprehensive Meta-Analysis (version 3.0). ${ }^{80}$ This software program allows for the automatic calculation of different data into correlation statistics. Additionally, standardized beta coefficients from individual studies were used as the correlation statistics. This decision was based on previous research showing that the correlation between the beta coefficient and correlation coefficient is linear, having a correlation of 0.84 if the coefficients reside in the interval $\pm 0.50 .{ }^{81}$ Studies that presented unstandardized beta-coefficients were first converted to standardized regression coefficients by multiplying the unstandardized coefficient by the ratio of the standard deviations of the independent variable and the dependent variable. Studies where unstandardized regression coefficients could not be converted to standardized regression coefficients and no other ES was provided were excluded from the MA.

Pooling of ES's. Results for the association between childhood obesity and selected CVD risk factors (SBP, DBP, TC, HDL, LDL, non-HDL, and TG) were pooled separately using random-effects, method-of-moments models. ${ }^{82}$ The correlation metric was converted to Fisher's z scale (Fisher's r-to-z transformation), and all analyses performed using the transformed values. ${ }^{79}$ These results included an overall effect estimate as well as $95 \%$ CI. ${ }^{82}$ If the $95 \%$ CI did not include zero (null $=0$ for correlation coefficient/Fishers $\mathrm{r}$ to $\mathrm{z}$ score), we considered our results to be statistically significant. We chose a random-effects model over a fixed-effect model because the former incorporates heterogeneity into the model. Random-effects models in MA assume that the true ESs are normally distributed and that studies are drawn from populations that differ from each other in ways that could impact the outcome. ${ }^{83}$ Moreover, several researchers suggest that when studies are gathered from the published literature, the random-effects model is generally a more appropriate model. ${ }^{79}$ This model takes into account both within and between-study variation while the fixed-effect model only takes into account within-study variation. In other words, a fixed-effect model assumes that all studies are drawn from a common population ${ }^{84}$ while a random-effects model does not. If there is no significant heterogeneity, the random-effects model 
and the fixed-effect model will produce similar results. ${ }^{59}$ If significant heterogeneity does exist, a random-effects model usually yields wider CI's. Forest plots were used to visually display the estimated ES of each study and their corresponding 95\% CI's. In addition, an overall pooled effect as well as 95\% CI's was generated. Furthermore, 95\% prediction intervals (PI's) were also calculated as a measure of dispersion in order to identify how the true value is likely to vary from one study to the next.

Stability and validity of changes in ES's. A primary purpose of MA is to investigate potential sources of heterogeneity of results in existing studies. ${ }^{85}$ For this study, we estimated heterogeneity for each outcome using the $I^{2}$ statistic. ${ }^{86,87}$ The $I^{2}$ statistic provides a useful summary of the impact and extent of heterogeneity, which helps in determining the robustness of drawing overall conclusions. ${ }^{86,87} I^{2}$ is calculated as $100 \% \times(\mathrm{Q}-\mathrm{df}) / \mathrm{Q}$, where $\mathrm{Q}$ is Cochran's heterogeneity statistic and df is the degrees of freedom. Statistical significance for $\mathrm{Q}$ was set at an alpha level of $\leq 0.10$ versus $\leq 0.05$ because the Q statistic suffers from power issues. ${ }^{87} \mathrm{~A}$ value of $0 \%$ indicates no observed heterogeneity while larger values show increasing heterogeneity. Negative values of $I^{2}$ are set to zero. ${ }^{87}$ For this study, heterogeneity for $I^{2}$ was classified as trivial $(0 \%-25 \%)$, low $(25.1 \%-50 \%)$, moderate $(50.1 \%-75 \%)$, or high $(75.1 \%-100 \%) .{ }^{87}$ The results were also interpreted with respect to the clinical implications of the degree of inconsistency as well as the magnitude and direction across studies, including the strength of evidence for heterogeneity. ${ }^{87}$

Publication bias is the tendency for authors to submit, and journals to accept and publish, manuscripts based on statistically significant findings. ${ }^{88}$ For this study, we followed the general guidelines of Sterne et al., ${ }^{89}$ for assessing publication bias, one of the potential reasons for what is known as "small-study effects", i.e., the tendency for smaller studies to show greater effects than larger studies. This includes a qualitative test, the funnel plot (inverse of standard error on the $\mathrm{y}$ axis and ES on the $\mathrm{x}$ axis) and a quantitative test, Egger's linear regression test. ${ }^{90}$ As recommended by Sterne et al., ${ }^{89}$ the test for funnel plot asymmetry was not used when there were fewer than 10 studies because power is usually low in identifying chance from real asymmetry. ${ }^{89}$ The Egger regression test is a regression of the standardized effect estimates against their precision (inverse standard error) and quantifies the funnel plot asymmetry by determining whether the intercept deviates significantly from zero. If the intercept is not significantly different from zero there is no evidence of funnel plot asymmetry. ${ }^{90-92}$ Influence analysis was used to examine the effects of each study on the overall results. This analysis excludes one study at a time in order to determine the influence of each study on the overall results. ${ }^{93}$ Cumulative $M A$, ranked by the year the study started, was used to examine the accumulation of findings over time by 
adding one study at a time and the results summarized as each new study is added. ${ }^{94}$ A sensitivity analysis was also performed by pooling the ESs from studies that only used childhood BMI as the exposure. This was performed in order to determine if any differences existed in the pooled results that included any definition for childhood exposure, including BMI.

Meta-regression. Because of missing data for different predictor variables from different studies, simple weighted least squares meta-regression (random-effects, method of moments approach) was used to examine the relationship between each outcome and selected covariates. Meta-regression is analogous to individual study regression except that the outcome variable is the effect estimate, i.e., unit of analysis is the study, rather than individual participant scores. ${ }^{59}$ This analysis is exploratory and used to indicate the magnitude and direction of association between variables as well as explore which factors, if any, best account for changes in outcomes. The slope of the regression coefficients along with their 95\% CIs were also calculated. CIs that did not cross zero were considered statistically significant. Planned covariates to examine a priori included: (1) country in which the study was conducted (USA, other), (2) bias due to loss to follow up, (3) type of analysis, (4) type of definitions used for adiposity, (5) exposure measure (self-report or not), (6) subject characteristics (sex, race/ethnicity), (7) studies that examined the association between childhood obesity and CVD risk factors while controlling or not controlling for adult adiposity, (8) time to follow up, (9) age categories of adults, (10) age categories of children, (11) comorbid conditions for both the child and the adult (diabetes, metabolic syndrome, etc.), (12) lipid lowering medication, (13) hypertensive medication, (14) family history of CVD, (15) smoking status/alcohol or drug use of both the child and the adult, (16) socio-economic status related variables, (17) diet, (18) physical activity, (19) fasting vs. non-fasting lipid profile, (20) child's pubertal status, (21) perinatal risk factors, and (22) study design. Where there was insufficient data (fewer than 3 results per group) for potential predictor variables, we performed a sensitivity analysis without the predictor to see if it had an effect on our overall findings. For categorical variables, less than three results for any one category were used as the cut-off for analysis. The results of the meta-regression tests need to be interpreted with caution since they are considered observational and exploratory inquiries, i.e., non-experimental comparisons, designed to generate hypotheses about potential sources of heterogeneity to be tested in original studies. ${ }^{59}$ In addition to statistical significance, the results of our study were also interpreted with respect to practical significance. 


\section{Results}

\section{Study Characteristics}

A general description of the characteristics of each study is shown in Table 3. Of the 4875 citations reviewed, a total of 23 were included in the $\mathrm{SR}^{4,95-116}$ and 21 in the MA., ${ }^{4,95-100,102-}$ ${ }^{110,112-116}$ A description of the search process, including the reasons for excluded studies, is shown in Figure 1. A list of all excluded studies and reasons for exclusion are shown in Additional file 2. The year that each study started varied considerably, ranging from 1923 to 1989 while the year that studies were published ranged from 1971 to 2014.

Studies were conducted in eleven different countries; six in the U.S., ${ }^{4,95,97,98,104,106}$ three in United Kingdom (England, Wales, Scotland and Newcastle), ${ }^{107,114,116}$ three in Finland, ${ }^{96,99,112}$ two in Australia, ${ }^{108,113}$ and one in Sweden, ${ }^{100}$ India, ${ }^{102}$ Lithuania, ${ }^{103}$ Poland, ${ }^{105}$ the Republic of Seychelles, ${ }^{109}$ Japan, ${ }^{110}$ and Six Solomon Island. ${ }^{115}$ Most studies used a prospective longitudinal study design except for two studies that used a retrospective study design. ${ }^{96,104}$ None of the studies used a case-cohort study design.

The length of follow-up for the studies ranged from 4.5 to 60 years. As most of the studies were prospective longitudinal studies, the number of subjects at baseline was often greater than the number of participants at follow-up due to loss to follow-up. Seven studies included information on loss to follow-up. ${ }^{4,99,100,102,103,108,113}$ Reasons for loss to follow-up included the following: (1) refused to participate, (2) inability to locate, (3) did not respond to contact, (4) participants out of country or town at time of follow-up, (5) death, (6) difficult to contact married girls in India who left their native village, (7) social disadvantage (less well educated and having lower family income). Two studies specified that participants who were lost to follow-up did not have significantly different childhood BMI's when compared to those who were available at follow-up in adulthood. ${ }^{4,116}$

As for the exposure, most studies used BMI as a measure of adiposity in childhood in addition to other measures used. ${ }^{4,96-100,102,103,105-110,112-116}$ However, two studies used relative overweight ${ }^{95}$ and sub-scapular skinfold thickness measures only. ${ }^{104}$ Most studies did not use a cut-off point to define childhood obesity, but rather, used childhood BMI as a continuous variable.

Sixteen studies examined the association between childhood obesity and adult SBP, ${ }^{4,95,97-}$ 100,102-104,106-110,113,116 14 examined the association between childhood obesity and adult DBP, 4,95,98100,102,103,106-110,113,116 8 examined the association between childhood obesity and adult TC, 4,95,98,110,112,114-116 5 examined the association between childhood obesity and adult 
LDL, 4,109,112,114,116 8 examined the association between childhood obesity and adult HDL, 4,98,99,109,112-114,116 and 9 examined the association between childhood obesity and adult TG. ${ }^{4,98,99,102,109,112,113,115,116}$ However, data from one study that assessed TG could not be used for MA because the study's main outcome was blood pressure and there was not enough information provided for TG to calculate an effects size. ${ }^{102}$ Only one study (Pereira et al., 2013) included data for the association between childhood adiposity and adult non-HDL cholesterol. ${ }^{111}$ However, the study by Pereira et al. was excluded from the MA due to the inability to retrieve data for metaanalytic use for other outcomes as well. Another study by Holland and colleagues was excluded from the MA for the same reason. ${ }^{101}$ Of the 21 studies, only six $(28.6 \%)$ included data on the association between childhood obesity and adult CVD risk factors while adjusting for adult BMI. ${ }^{4,96,105,107,109,116}$ Two of these six studies reported adjusted associations only. ${ }^{96,105}$

\section{Participant Characteristics}

As previously stated, a description of the participant characteristics is shown in Table 3. The majority of studies included information on both males and females, ${ }^{97,100,103-106,109,110,112,115,116}$ two were limited to men only, ${ }^{95,102}$ while 9 included combined data for both men and women. ${ }^{4,96,98,99,107-109,113,114}$ No study was limited to women. One study included combined results as well as results according to sex. ${ }^{109}$ However, for the current MA, we used the results reported according to sex. ${ }^{109}$ Moreover, while one study had data on both males and females, data from only males was used for the current MA because the regression model for females included change of BMI over time i.e., from childhood to adulthood. ${ }^{97}$

The participants' ages at baseline when the exposure was measured ranged from 2 to 18 years. The age at follow-up when the outcome was measured also varied substantially between studies, ranging from 19 to 62 years. Some studies used one childhood age or average of a range of childhood ages as their exposure, while some studies categorized children based on different age groups. Furthermore, some studies used longitudinal data for the same children over time.

Most of the studies provided some level of information on comorbid conditions of adults. These included hypertension, ${ }^{95}$ arteriosclerotic heart disease,${ }^{95} \mathrm{CV}$ renal disease, ${ }^{95}$ coronary heart disease, ${ }^{96}$ diabetes, insulin or glucose levels, ${ }^{4,99,109}$ metabolic syndrome, ${ }^{100}$ medication for heart diseases, ${ }^{107}$ medication for hypertension, ${ }^{9,105,107}$ and uric acid level. ${ }^{109}$ One study excluded participants who were on hypertension (HT) medication, ${ }^{104}$ whereas one study found no difference in any of the analyses after performing sensitivity analysis and excluding those subjects who were taking cholesterol-lowering drugs. ${ }^{114}$ For women, additional information on the use of oral contraceptives, menstruation, menopausal status, and use of hormonal replacement 
therapy was provided by a five studies. ${ }^{105-107,112,114}$ Family history of CVD or CVD risk factors was available in 2 studies. ${ }^{104,106}$ Information on smoking, alcohol, and drug use was provided by six studies. ${ }^{100,102,106,107,112,113}$ Perinatal risk factors such as birth weight and/or gestational age were presented in approximately one third of the studies. ${ }^{97,99,107,108,110,114,116}$ Information on diet and physical activity was provided by 4 studies, ${ }^{100,107,112,113}$ while information on the child's pubertal status was provided by one study. ${ }^{103}$ It is important to note that these variables were not necessarily adjusted for in the analyses performed by the individual studies.

\section{Risk of Bias Assessment}

Overall study-level risk of bias results are shown in Figure 2 while results for each item from each study are shown in Additional file 3. More than 50\% of the studies did not provide an adequate description of participant characteristics while almost $70 \%$ did not describe any efforts to address potential sources of bias, explain how loss to follow-up was addressed, or provide reasons for non-participation at each stage. More than $80 \%$ did not explain how missing data was addressed. At the study level, nine studies had more than one third of items that were at an increased risk of bias for reporting of items for observational studies. ${ }^{95,97,101,102,104-106,112,116}$

\section{Primary Outcomes}

The results of the random-effects MA for the association between childhood obesity and adult CVD risk factors are presented below. The forest plots displaying the effect estimates along with the 95\% CI for each outcome is shown in Figures 3-14. The forest plots for influence analyses, cumulative MA, and funnel plots are shown in Additional file 4. Exploratory randomeffects meta-regression analyses for the association between childhood obesity and adult CVD risk factors and selected covariates (categorical and continuous) in which adequate data were available are shown in Additional file 5.

Systolic Blood Pressure (unadjusted for adult adiposity). Overall, there was a statistically significant and positive association between childhood adiposity and adult SBP (Table 4 and Figure 3). Heterogeneity was also statistically significant and large. However, 95\% PIs were non-significant. Funnel plot results for small-study effects showed a lack of asymmetry and were reinforced by a lack of statistical significance based on Egger et al.'s regression intercept test $(\mathrm{p}=0.42)$. With each study deleted from the model once, results remained statistically significant across all deletions. The associations ranged from approximately 0.10 to 0.11. Cumulative MA, ranked by the year the study started, demonstrated that results have been 
statistically significant since examining the birth cohort of 1958. Random-effects meta-regression revealed statistically significant evidence for an association between pooled ES (Fisher's $r$ to $z$ score for the association between childhood obesity and adult SBP) and baseline age ( $\beta=0.01, \mathrm{p}$ $=0.01 ; I^{2}$ reduced from $91 \%$ to $88 \%$ ), studies conducted in the United States versus other countries $(\beta=0.07, p=0.05)$ and studies that used BMI versus alternative methods for assessing adiposity $(\beta=0.05, \mathrm{p}=0.05)$.

Systolic Blood Pressure (adjusted for adult adiposity). When examining studies that adjusted for adult BMI, a statistically significant and negative association was observed between childhood adiposity and adult SBP (Table 4 and Figure 4). Heterogeneity was also statistically significant and considered large. However, 95\% PIs were non-significant. Because there were less than 10 studies, small-study effects were not assessed. With each study deleted from the model once, results remained statistically significant across all deletions. The associations ranged from approximately -0.10 to -0.13 . Cumulative MA, ranked by the year the studies started, demonstrated that results have been statistically significant since the first study was conducted in 1934. Random-effects meta-regression revealed statistically significant evidence for an association between pooled ES (Fisher's $r$ to $\mathrm{z}$ score for the association between childhood obesity and adult SBP for studies that adjusted for adult BMI) and follow-up age $(\beta=-0.005, p=$ 0.002 ; $\mathrm{I}^{2}$ reduced from $89 \%$ to $69 \%$ ) as well as length of follow-up (follow-up age - baseline age) $\left(\beta=-0.004, \mathrm{p}=0.008 ; I^{2}\right.$ reduced from $89 \%$ to $\left.76 \%\right)$.

Diastolic Blood Pressure (unadjusted for adult adiposity). Overall, there was a statistically significant and positive association between childhood adiposity and adult DBP (Table 4 and Figure 5). Heterogeneity was also statistically significant and large. However, 95\% PIs were non-significant. Funnel plot results for small-study effects showed a lack of asymmetry and were reinforced by a lack of statistical significance based on Egger et al.'s regression intercept test $(\mathrm{p}=0.37)$. With each study deleted from the model once, results remained statistically significant across all deletions. The associations ranged from 0.094 to 0.113 . Cumulative MA, ranked by the year the study started, demonstrated that results have been significant since examining the birth cohort of 1966. Random-effects meta-regression revealed statistically significant evidence for an association between pooled ES (Fisher's $\mathrm{r}$ to $\mathrm{z}$ score for the association between childhood obesity and adult DBP $)$ and baseline age $\left(\beta=0.01, \mathrm{p}=0.01 ; I^{2}\right.$ reduced from $90 \%$ to $87 \%$ ). 
Diastolic Blood Pressure (adjusted for adult adiposity). When examining studies that adjusted for adult BMI, a statistically significant and negative association was observed between childhood adiposity and adult DBP (Table 4 and Figure 6a). Heterogeneity was also statistically significant and considered large. However, 95\% PIs were non-significant. Because there were less than 10 studies, small-study effects were not assessed. The results of the influence analysis showed that when two studies (Koziel et al, 2011 and Li et al., 2007) were deleted separately from the model, results were slightly non-significant. ${ }^{105,107}$ The results remained statistically significant when deleting the rest of the studies individually. The associations ranged from approximately -0.081 to -0.131 . Cumulative MA, ranked by the year the studies started, demonstrated that results have been statistically significant since the first study was conducted in 1947. Random-effects meta-regression revealed statistically significant evidence for an association between pooled ES (Fisher's $r$ to $z$ score for the association between childhood obesity and adult DBP for studies that adjusted for adult BMI) and follow-up age ( $\beta=-0.006, p$ $<0.0001 ; I^{2}$ reduced $92 \%$ to $58 \%$ ), and length of follow-up $\left(\beta=-0.006, \mathrm{p}<0.0001 ; I^{2}\right.$ reduced from $92 \%$ to $56 \%$ ).

Total Cholesterol (unadjusted for adult adiposity): Overall, there was a positive association between childhood adiposity and adult TC (Table 4 and Figure 7). However, the association was not statistically significant. The $95 \%$ PIs were also non-significant. The heterogeneity was statistically significant and large. Because there were less than 10 studies, small-study effects were not assessed. With each study deleted from the model once, results remained statistically non-significant across all deletions. The associations ranged from approximately -0.01 to 0.02 . Cumulative meta-analysis, ranked by the year the study started, demonstrated that results have been negative since the start of the first study in 1923 up to the sixth study that started in 1970 after which the cumulative results showed a positive association. Random-effects meta-regression revealed statistically significant evidence for an association between pooled ES (Fisher's $r$ to $z$ score for the association between childhood obesity and adult TC) and follow-up age ( $\beta=-0.004, p=0.04 ; I^{2}$ reduced from $91 \%$ to $76 \%$ ), and length of followup $\left(\beta=-0.004, p=0.01 ; I^{2}\right.$ reduced from $91 \%$ to $\left.67 \%\right)$.

Total Cholesterol (adjusted for adult adiposity). When examining studies that adjusted for adult BMI, a negative and non-significant association was observed between childhood adiposity and adult TC (Table 4 and Figure 8 ). The 95\% PIs were non-significant as well. Heterogeneity was statistically significant and considered large. Because there were less than 10 
studies, small-study effects were not assessed. With each study deleted from the model once, results remained statistically non-significant across all deletions except when one study by Barker et al., 2005 was deleted, resulting in statistically significant results. The associations ranged from approximately -0.036 to -0.090 . Cumulative meta-analysis, ranked by the year the studies started, demonstrated that results have been negative since examining the birth cohort of 1947. Randomeffects meta-regression revealed statistically significant evidence for an association between pooled ES (Fisher's $r$ to z score for the association between childhood obesity and adult TC for studies that adjusted for adult BMI) and baseline age $\left(\beta=-0.32, \mathrm{p}<0.0001 ; I^{2}\right.$ reduced from $86 \%$ to $0 \%)$ and $\operatorname{sex}\left(\beta=-0.32, \mathrm{p}<0.0001 ; I^{2}\right.$ reduced from $77 \%$ to $\left.35 \%\right)$.

Low-density Lipoprotein (unadjusted for adult adiposity): Overall, there was a positive association between childhood adiposity and adult LDL (Table 4 and Figure 9). However, the results were not statistically significant. The 95\% PIs were non-significant as well. Heterogeneity was statistically significant and large. Because there were less than 10 studies, small-study effects were not assessed. With each study deleted from the model once, results remained statistically non-significant across all deletions. The associations ranged from approximately 0.095 to -0.085 . Cumulative meta-analysis, ranked by the year the study started, demonstrated that results have been statistically non-significant since examining the birth cohort of 1947. The results were negative with the first three studies added and became positive after the fourth and fifth studies were included. Random-effects meta-regression revealed statistically significant evidence for an association between pooled ES (Fisher's $r$ to $\mathrm{z}$ score for the association between childhood obesity and adult LDL) and follow-up age $(\beta=-0.005, \mathrm{p}=0.0001$; $I^{2}$ reduced from $94 \%$ to $39 \%$ ), and length of follow-up $\left(\beta=-0.004, p=0.005 ; I^{2}\right.$ reduced from $94 \%$ to $59 \%)$.

Low-density Lipoprotein (adjusted for adult adiposity): When examining the three studies that adjusted for adult BMI, a statistically significant and negative association was observed between childhood adiposity and adult LDL (Table 4 and Figure 10). Heterogeneity was also statistically significant and considered large. However, 95\% PIs were significant. Because there were less than 10 studies, small-study effects were not assessed. With each study deleted from the model once, results remained statistically significant across all deletions. The associations ranged from approximately -0.07 to -0.09 . Cumulative meta-analysis, ranked by the year the studies started, demonstrated that results have been statistically significant since the first study was conducted in 1947. Random-effects meta-regression revealed no statistically 
significant evidence for an association between pooled ES (Fisher's $r$ to $\mathrm{z}$ score for the association between childhood obesity and adult LDL for studies that adjusted for adult BMI) and sex. None of the other variables were examined because of insufficient data.

High-density Lipoprotein (unadjusted for adult adiposity): Overall, there was a statistically significant and negative association between childhood adiposity and adult HDL (Table 4 and Figure 11). Heterogeneity was also statistically significant and large. However, 95\% PIs were non-significant. Because there were less than 10 studies, small-study effects were not assessed. With each study deleted from the model once, results remained statistically significant across all deletions except when one study by Schmidt et al., 2011 was deleted. Deleting this study resulted in a negative non-significant association. The associations ranged from approximately -0.04 to -0.07 . Cumulative meta-analysis, ranked by the year the study started, demonstrated that results have been statistically significant since examining the birth cohort of 1985. Random-effects meta-regression revealed no statistically significant evidence for an association between pooled ES (Fisher's $r$ to $\mathrm{z}$ score for the association between childhood obesity and adult HDL) for all the covariates examined.

High-density Lipoprotein (adjusted for adult adiposity): When examining studies that adjusted for adult BMI, a positive but non-significant association was observed between childhood adiposity and adult HDL (Table 4 and Figure 12). Heterogeneity was statistically significant and considered large. However, 95\% PIs were non-significant. Because there were less than 10 studies, small-study effects were not assessed. With each study deleted from the model once, results remained statistically non-significant across all deletions. The associations ranged from approximately -0.009 to 0.079 . Cumulative meta-analysis, ranked by the year the studies started, demonstrated that results have been statistically non-significant since the first study was conducted in 1947. However, it is important to note that these results are based on only four studies and the direction of association changed from positive to negative and then positive over time. Random-effects meta-regression revealed statistically significant evidence for an association between pooled ES (Fisher's $r$ to $\mathrm{z}$ score for the association between childhood obesity and adult HDL for studies that adjusted for adult BMI) and follow-up age $(\beta=-0.007, p=$ $0.004 ; I^{2}$ reduced from $94 \%$ to $\left.74 \%\right)$, length of follow-up, $\left(\beta=-0.007, \mathrm{p}=0.004 ; I^{2}\right.$ reduced from $94 \%$ to $76 \%$ ) and sex (males vs. female, $\beta=-0.13, \mathrm{p}=0.01 ; I^{2}$ reduced from $66 \%$ to $59 \%$ ). 
Triglycerides (unadjusted for adult adiposity): Overall, there was a statistically significant and positive association between childhood adiposity and adult TG (Table 4 and Figure 13). Heterogeneity was also statistically significant and large. However, 95\% PIs were non-significant. Because there were less than 10 studies, small-study effects were not assessed. With each study deleted from the model once, results remained statistically significant across all deletions except when Freedman et al., 2001 and Schmidt et al., 2011 were deleted. The associations ranged from approximately 0.07 to 0.11 . Cumulative meta-analysis, ranked by the year the study started, demonstrated that results have been statistically significant since examining the birth cohort of 1985. Random-effects meta-regression revealed statistically significant evidence for an association between pooled ES (Fisher's $r$ to $z$ score for the association between childhood obesity and adult TG) and follow-up age $(\beta=-0.001, p<0.0001$; $I^{2}$ reduced from $83 \%$ to $\left.50.35 \%\right)$, and length of follow-up $\left(\beta=-0.007, \mathrm{p}=0.0001 ; I^{2}\right.$ reduced from $83 \%$ to $50 \%$ ).

Triglycerides (adjusted for adult adiposity): When examining studies that adjusted for adult BMI, a negative association was observed between childhood adiposity and adult TG (Table 4 and Figure 14). However, the association was not statistically significant. The 95\% PIs were non-significant as well. Heterogeneity was statistically significant and considered large. Because there were less than 10 studies, small-study effects were not assessed. With each study deleted from the model once, results remained statistically non-significant across all deletions. The associations ranged from approximately -0.05 to -0.13 . Cumulative meta-analysis, ranked by the year the studies started, demonstrated that results have been statistically non-significant since examining the cohort of 1947. Random-effects meta-regression revealed statistically significant evidence for an association between pooled ES (Fisher's $r$ to $z$ score for the association between childhood obesity and adult TG for studies that adjusted for adult BMI) and follow-up age ( $\beta=$ $0.006, \mathrm{p}=0.05 ; I^{2}$ reduced from $95 \%$ to $\left.92 \%\right)$, length of follow-up, $\left(\beta=0.004, \mathrm{p}=0.02 ; I^{2}\right.$ reduced from $95 \%$ to $90 \%$ ) and sex (male vs. females, $\beta=0.05, \mathrm{p}=0.002 ; I^{2}$ reduced from $60 \%$ to $45 \%$ ).

Non-High-density Lipoprotein. Our study was not able to perform MA on the association between childhood obesity and non-HDL levels due to lack of data.

\section{Sensitivity Analysis}

The results of the sensitivity analysis for all the outcomes using only BMI as the 
exposure showed that childhood obesity is significantly and positively associated with adult SBP $(\mathrm{Zr}=0.10 ; 95 \% \mathrm{CI}: 0.06,0.13), \mathrm{DBP}(\mathrm{Zr}=0.11 ; 95 \% \mathrm{CI}: 0.07,0.14)$, and $\mathrm{TG}(\mathrm{Zr}=0.10 ; 95 \%$ CI: $0.02,0.17)$, and significantly and inversely associated with adult $\mathrm{HDL}(\mathrm{Zr}=-0.06 ; 95 \% \mathrm{CI}$ $0.11,-0.01)$ (Table 5).

\section{Discussion}

\section{Findings}

The purpose of this study was to conduct a SR and MA of studies that have examined the association between childhood obesity and adult CVD risk factors (SBP, DBP, TC, HDL, LDL, non-HDL, and TG). The overall, unadjusted findings suggest that childhood obesity is significantly and positively associated with adult SBP, DBP, and TG and significantly and negatively associated with adult HDL. This interpretation is supported by: (1) $95 \%$ CI for overall results that do not include the null, (2) consistency of overall results when each study was deleted from the model once (influence analysis), (3) significance of results over a long time period in which the included studies were conducted (cumulative meta-analysis), and (4) nonsignificant small study effects.

When examining studies that adjusted for adult obesity, the overall findings suggest that the association was significant and negative for SBP, DBP, and LDL while the associations between childhood obesity and adult HDL and TG became non-significant when adult BMI was accounted for. However, it is important to point out that less than one third of studies adjusted for adult adiposity measures. ${ }^{4,96,105,107,109,116}$ For the studies that adjusted for adult BMI, the associations became reversed, suggesting that the association between childhood adiposity and adult CVD risk factors is potentially mediated by adult adiposity. The correlation coefficient for childhood adiposity from childhood to adulthood ranged from 0.3 to 0.8 (mean $=0.6, \mathrm{SD}=0.1$ ), demonstrating a medium to strong tracking of adiposity across the lifespan. This is also consistent with previous research suggesting that children who are obese have a $40 \%-80 \%$ chance of becoming overweight or obese adults..$^{21-23}$

Several factors need to be taken under consideration when examining the results of this study. First, we used random-effects models that incorporate heterogeneity into the analysis. However, based on the fixed-effect model, we observed a moderate to large amount of heterogeneity in all the outcomes assessed. While a random-effects model incorporates heterogeneity into the analysis, it does not explain the sources of heterogeneity. Second, the $95 \%$ PIs were not statistically significant as they overlapped the null (0). As previously noted, the $95 \%$ 
CI are centered around the point estimate of the between study variation. A 95\% PI is based on the random mean effect, and is an interval/range in which $95 \%$ of the true study's expected effects are found. This is the area where the true effect measure of the new study lies, or simultaneously the predicted parameter value in a new study can be deduced, thus giving more confidence in the overall results of the study. ${ }^{117,118}$ Third, many studies were considered to be at an increased risk of bias for the quality of reporting on several items of the STROBE instrument (Figure 2 and Additional file 3). More specifically, nearly $70 \%$ of the studies were considered to be at a high risk of bias for the following elements: (1) describing any efforts to address potential sources of bias, (2) explaining how missing data were addressed, (3) explaining how loss to follow-up was addressed, (4) describing any sensitivity analyses, (5) providing reasons for nonparticipation at each stage, (6) considering the use of a flow diagram, (7) considering translating estimates of relative risk into absolute risk for a meaningful time period, and (8) not providing adequate information on participants characteristics. Fourth, loss to follow-up is one of the main sources of bias in longitudinal studies. The potential bias occurs when the participants who are lost to follow-up are different from participants who remain in the study. ${ }^{119}$ Research suggests that more than a $20 \%$ loss to follow-up is a potential threat to the internal validity of the study. ${ }^{120}$ Only seven studies included information on loss to follow-up..$^{4,99,100,102,103,108,113}$ Lastly, some of the associations observed in the exploratory covariates from the results of the meta-regression analyses suggest that some factors may potentially impact the overall conclusions. These include different factors for different outcomes. The significant factors included: (1) baseline age for adult SBP and DBP, (2) follow up age for TC, LDL, and TG (3), length of follow-up for TC, LDL, and TG, (4) country study was conducted for SBP, and (5) type of exposure used to assess adiposity for SBP. For the studies that adjusted for adult BMI, the factors included (1) baseline age for TC, (2) follow up age for SBP, DBP, HDL, and TG (3) length of follow-up for SBP, DBP, HDL, and TG and (4) sex for TC, HDL, and TG. The statistically significant findings of the meta-regression are discussed below.

The results from the meta-regression analysis revealed that the association between childhood obesity and adult SBP and DBP increases as the baseline age increases. For TC, LDL, and TG the association decreases as the follow-up age and length of follow-up increases. The association was higher in U.S. compared to other countries, and higher for studies that used BMI to measure the exposure compared to other measures of adiposity for SBP only. For studies that adjusted for adult BMI the association between childhood obesity and adult TC increases as the baseline age increases. For SBP, DBP, and HDL the association decreases as the follow-up age and length of follow-up increases. The association was lower in males compared to females for 
TC and HDL, and higher in males compared to females for TGs. However, the results found one unusual finding for the association between childhood obesity and adult TG (adjusted for adult BMI) that increased with the increase in the follow-up age/length of follow-up. We hypothesize that this odd finding could have been due to the play of chance given all the tests that were conducted. However, the results of the meta-regression tests should be interpreted with caution since they are considered observational and exploratory in nature, i.e., non-experimental comparisons, designed to generate hypotheses about potential sources of heterogeneity. ${ }^{59}$ Thus, these would need to be tested and confirmed in original studies.

\section{Critical Evaluation of Results compared to Previous Systematic Reviews and Meta-analysis on the Topic}

As discussed earlier, four SRs published on this topic from 2010-2012 provided qualitative evidence but did not provide any quantitative evidence on the association between childhood obesity and adult CVD risk factors (BP and lipid profile). While one quantitative analysis was conducted four years ago on this topic, it was limited to a select four cohorts only and thus, not considered a true MA ${ }^{40}$ The results of our study as well as similarities and differences from these previously conducted SRs and MA are discussed below.

Previous systematic reviews ${ }^{18,37-39}$ The SR by Lloyd and colleagues ${ }^{37}$ found little evidence that childhood obesity is an independent risk factor for adult SBP and DBP. They concluded that the relationships observed are dependent on the tracking of BMI from childhood to adulthood. They found that the positive association between childhood BMI and adult blood pressure was attenuated or became negative when taking into account adult BMI. The results of our study are in congruence with the findings of this SR. Notably, the second SR by Lloyd and colleagues ${ }^{18}$ also found little evidence that childhood obesity is an independent risk factor adult TC, LDL, HDL, and TG. They found that the association between childhood BMI and adult lipid levels was attenuated or inversed when taking into account adult BMI. The results of our study are also consistent with the findings of this SR.

The SR by Reilly and colleagues ${ }^{39}$ reported a significant and positive association between childhood adiposity and adult HT. However, the SR did not mention if the studies included in the review adjusted for adult adiposity. The SR by Park and colleagues ${ }^{38}$ also found a significant and positive association between childhood adiposity and adult HT. Two out of five studies described in this $\mathrm{SR}^{38}$ that adjusted for adult BMI found no association. We believe that the present study is perhaps answering a different research question (SBP and DBP versus HT). However, since HT is 
based on both SBP and DBP, it appeared important to mention these differences in findings. ${ }^{38,39}$ Along those lines, Park and colleagues suggested in their SR that since adult BMI is on the causal pathway for the association between childhood obesity and adult disease, adjusting for adult BMI has methodological limitations. One of the main limitations being that adjusting for variables on the casual pathway can lead to spurious associations (over-adjustment biases) that can draw estimates towards the null. The study also cited a simulation study that showed that a true positive association between birth weight and adult BP was diminished after adjusting for current adult weight status, something that could be reversed if the correlation between birth weight and current weight was increased. ${ }^{121}$ As childhood adiposity and adult adiposity are strongly correlated, this can be a potential issue. However, this debate has been both criticized by other researchers as well as supported. ${ }^{122-124}$ Some of the main differences of our study from these earlier SRs include: (1) combining the ESs of the included studies using the meta-analytic approach, (2) using SBP and DBP instead of HT, ${ }^{38,39}$ (3) performing MA on systematically finding 16 studies for SBP and 14 studies for DBP, 8 studies each for TC, HDL, and TG, and 5 studies for LDL, (4) including additional studies published up to June, 2015, (5) utilizing numerous definitions for childhood adiposity (exposure), (6) excluding studies that examined change of exposure from childhood to adulthood, ${ }^{18,37}$ (7) excluding special populations, ${ }^{18,37}(8)$ excluding gestational hypertension ${ }^{39}$ and, (9) excluding studies that used self-reported outcomes. ${ }^{39}$

Previous Meta-analysis ${ }^{40}$ The MA by Juonala and colleagues used data from four cohorts: the Bogalusa Heart Study (BHS) the Muscatine Study (MS), the Childhood Determinants of Adult Health (CDAH) study, and the Cardiovascular Risk in Young Finns Study (YFS). The results from the random effects MA showed a significant association between childhood obesity in predicting the following adult CVD outcomes using risk ratios: $\mathrm{HT}=2.1(95 \% \mathrm{CI}: 1.8,2.5)$, $\mathrm{LDL}=1.6(95 \% \mathrm{CI}: 1.3,2.0)$, high risk $\mathrm{HDL}=1.7(95 \% \mathrm{CI}: 1.5,1.9)$, and $\mathrm{TG}=1.8(95 \% \mathrm{CI}:$ $1.5,2.2)$. The direction of effect for the association between childhood obesity and adult CVD risk factors in the current MA is consistent with the previous meta-analytic work by Juonala et al. ${ }^{40}$ Juonala and colleagues used pooled data from all 4 studies and showed that childhood obesity was significantly associated with HT even after adjustment for adult obesity (relative risk, $1.5 ; 95 \%$ CI: $1.1,2.1 ; \mathrm{P}=0.009)$. For dyslipidemias, the effect of childhood adiposity was reduced and became non-significant when adult obesity was taken into account. The results of our MA are consistent with the pooled results for dyslipidemias. However, this previous study was not a true MA. ${ }^{40}$ Some of the main differences in our study compared to this previous 
investigation include: (1) using a systematic approach to find studies published until June 2015 that have examined these selected associations, (2) using SBP and DBP instead of HT, (3) examining the association for TC, (4) finding a positive but non-significant association for LDL, (5) performing MA on systematically finding 16 studies for SBP and 14 studies for DBP, 8 studies each for TC, HDL and TG and 5 studies for LDL, (6) performing MA on studies that adjusted for BMI, and (7) utilization of numerous definitions for childhood adiposity (exposure).

\section{Implications for Research}

The results of the current SR with MA have several implications for reporting of future longitudinal studies. First, based on the STROBE instrument, it is recommended that future longitudinal studies improve their reporting with respect to several potential sources of bias. These include: (1) describing any efforts to address potential sources of bias, (2) explaining how missing data were addressed, (3) explaining how loss to follow-up was addressed, (4) describing any sensitivity analyses conducted, (4) reporting the numbers of individuals at each stage of the study, (5) providing reasons for non-participation at each stage, (6) considering the use of a flow diagram, (7) describing the characteristics of study participants, and (8) considering translating estimates of relative risk into absolute risk for a meaningful time period. Because longitudinal studies have a criterion for initially selecting participants that chose to participate or not, have varied response rates, different numbers of participants at baseline and follow-up, as well as varied participation and response rates at follow-up time point(s), it is important to demonstrate this information using a flow diagram. However, only one study used a flow diagram. Therefore, it is suggested that future longitudinal studies include a flow diagram in order to clearly demonstrate their study design, participation and response rates. Second, complete information on the population characteristics should be presented (usually in Table 1 of most articles). Unfortunately, more than $50 \%$ of the studies did not provide adequate information on the population characteristics. Third, as loss to follow-up is a potential threat to the internal validity of the study, ${ }^{120}$ this information should also be provided. Only seven studies included information on loss to follow-up. Fourth, only one study reported on the association between childhood obesity and adult non-HDL. This is important since non-HDL has been shown to be better marker of risk for coronary artery disease and stroke compared to LDL. ${ }^{42,43}$ It is suggested that future studies collect and report this information. Fifth, only one third of the studies adjusted for adult adiposity. Given the former, it would appear prudent to suggest that future studies collect this information and present both crude and adjusted associations. Sixth, some studies presented results with unstandardized regression coefficients only. Among those studies that only provided 
unstandardized regression coefficients, we were able to calculate standardized regression coefficients using the standard deviations of the exposure and the outcome. However, there were some studies where the standard deviations were not provided. As a result, we were unable to use data from these studies for our MA. Given the former, it is suggested that future studies provide information for both standardized and unstandardized regression coefficients. Seventh, while the majority of studies included information on males and females, ${ }^{97,100,103-106,109,110,112,115,116}$ two were

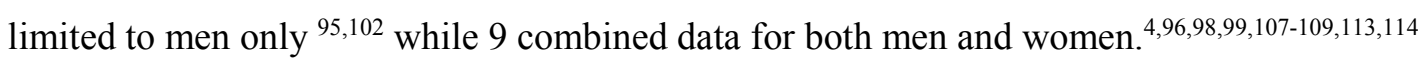
Given biological differences between men and women, it would appear plausible to suggest that future studies include separate as well as combined results for both men and women. Eighth, only one study provided information on the pubertal status of children. It is important to collect and adjust for pubertal status in future studies as previous studies have shown an association between childhood obesity and pubertal developmental, ${ }^{125}$ as well as between pubertal timing and adult cardio-metabolic risk factors. ${ }^{126}$ Ninth, most studies used BMI as a measure of adiposity for the childhood exposure. ${ }^{4,96-100,102,103,105-110,112-116}$ However, since prior research has shown that BMI is not an ideal marker for adiposity, ${ }^{47,48}$ it is suggested that future studies collect information on additional markers for adiposity, for example percent body fat, in addition to BMI. Lastly, the negative associations in the adjusted analysis for all outcomes, and a positive association for HDL provides the basis for future research to explore whether children at the lower end of BMI during childhood are at a higher risk for developing CVD risk factors compared to children at the higher end of the BMI spectrum during childhood and after adjusting for adult BMI.

\section{Implications for Practice}

The results of the current MA have relevant implications for practice. Overall, it appears that childhood obesity is positively associated with adult SBP, DBP, and TG and negatively associated with adult HDL. Given the former, prevention of childhood obesity should remain a priority for public health interventions for preventing negative health outcomes during childhood as well as reducing the burden of adult obesity. Furthermore, this study adds to the argument that obese children who become normal weight adults are probably not at any higher risk of CVD risk factor development if they become non-obese in adulthood. However, these findings need to be interpreted with caution given that only one third of the studies adjusted for adult BMI.

\section{Strengths of the Study}

To the best of our knowledge, this is the first MA and most recent SR that has systematically appraised studies examining these associations. The present study is based on a 
greater number of studies (published up to June 2015) that included both the crude associations as well as studies that adjusted for adult adiposity. This study also included any definition of adiposity measure that was utilized for the exposure. Research has shown that BMI is not an ideal marker for adiposity ${ }^{47,48}$ and including other definitions or classifications of adiposity helped us in identifying other potentially eligible studies that have looked at this association. Although we performed the main MA using studies that utilized varied childhood adiposity measure, the results of our sensitivity analysis using only BMI as the exposure showed similar findings (Table 5). In addition, we also used SBP and DBP instead of HT to examine for independent associations between childhood obesity and components of HT (i.e. high SBP and/or DBP). Lastly, we performed meta-regression analysis on covariates that may potentially impact this association and to inform future research on these factors.

\section{Limitations of the Study}

The results of the current MA should be viewed with respect to the following potential limitations. First, only one third of the included studies adjusted for adult BMI. Second, some of the pre-planned analyses to identify sources of heterogeneity were not performed due to lack of data. Third, the sample sizes for many of the analyses were probably underpowered to find a true effect. Fourth, due to the small sample sizes for some analyses, small-study effects (e.g. potential publication bias) were not conducted. Fifth, the a priori plan of the current study did not include an age range for childhood exposure. A post hoc decision was made to limit the age range from 219. However, due to including this age range the investigative team faced an additional challenge i.e. some outcomes were assessed where the mean age of the participants at the follow-up time point was greater than 19 but the age range included less than 19 year old participants as well. There were four studies in our review where the mean age was 27 but the age ranged from 18 $37,{ }^{4}$ mean age 24 and age ranged from $18-26,{ }^{102}$ mean age 21 and age ranged from 18-24, ${ }^{108}$ and mean age 20 and age ranged from 18-21. ${ }^{109}$ Sixth, MA cannot make up for the poor quality of the original studies and is inherently vulnerable to the biases in the original studies. Seventh, in order to retrieve information on missing data, we contacted the corresponding authors via email in order to obtain necessary information. Nearly $30 \%$ of the corresponding authors replied but no author provided any additional information. Eighth, we excluded studies that were not published in the English language, which could potentially introduce language bias. However, we do not believe that this was a major problem since previous research has shown that meta-analyses that restrict studies by language overestimate the effect of the outcomes by only $2 \% .{ }^{66} \mathrm{Ninth}$, like any $\mathrm{SR}$, literature search bias is a potential problem where some relevant literature is not identified 
during the search process. However, we performed an exhaustive search according to pre-defined criteria examining nearly 5000 citations. Thus, we expect this bias to be minimal. Finally, given the large number of analyses we conducted, one or more of our findings may have been due to the play of chance. However, no adjustment for multiple tests were made given that we did not want to miss potentially important findings that could be tested in future original studies. ${ }^{127}$ Though the study had several limitations, to the best of the investigative team's knowledge, this is the first MA to examine the association between childhood obesity and adult CVD risk factors as well as examine this association in studies that adjusted for adult BMI.

\section{Conclusion}

The results of the current MA suggest that childhood obesity is significantly and positively associated with adult SBP, DBP, and TG and negatively associated with adult HDL. However, additional, well-designed, longitudinal studies with improved reporting as well as data analysis that includes both unadjusted and adjusted associations for adult adiposity are needed before any definitive conclusions can be reached. 


\section{Chapter 5: Discussion}

\section{Summary of Key Findings}

\section{Overview}

The current set of studies aimed to examine three distinctive distal determinants of cardiovascular (CVD) risk factors during childhood and adulthood. We focused on two distal perinatal factors (birth weight of the infant and reported history of breastfeeding) and later childhood and maternal CVD risk factors for the first two studies. For the third study we focused on childhood obesity as the distal determinant of adult CVD risk factors. The CVD risk factors included: (1) blood pressure including both systolic blood pressure (SBP) and diastolic blood pressure (DBP), (2) lipids including total cholesterol (TC), high-density lipoprotein cholesterol (HDL), non-high-density lipoprotein cholesterol (non-HDL), low-density lipoprotein cholesterol (LDL), and triglycerides (TG), and (3) obesity as assessed by body mass index (BMI). Previous studies that have examined these distal exposures and CVD risk factors at various life stages typically have shown inconsistent results. Thus, the overall objective of this project as a whole was to determine the associations between perinatal risk factors (birth weight and breastfeeding) and childhood and maternal cardiovascular health, and determine the relationship between childhood obesity and adult CVD risk factors.

For the first two studies we used data from three projects, including West Virginia (WV) Birth Certificates, the Working in Appalachia to Track High Birth Score, Critical Congenital Heart Disease and Hearing Loss (WATCH)/Birth Score project, and the Coronary Artery Risk Detection in Appalachian Communities (CARDIAC) project. For the first study, children participating in the Birth-Score project (all of whom are merged with the Birth Certificate data) born in 1994-2000 were merged with data collected by the CARDIAC project in years 2004-2010 and restricted to full-term birth infants only $(\mathrm{N}=19,583)$. For the second study, children participating in the WATCH/Birth-Score project at birth (all of whom are merged with the Birth Certificate data) born between in 1997-2004 were merged with data collected by the CARDIAC project in years 2008-2014 ( $\mathrm{N}=10,457)$. For the third study we conducted a systematic review and meta-analysis to critically evaluate the evidence regarding the relationship between childhood obesity and adult CVD risk factors. A total of 4,840 citations were reviewed utilizing numerous resources. The inclusion criteria were: (1) longitudinal study-design, (2) childhood exposure and adult outcomes collected on the same individual over time, (3) childhood obesity, as defined by 
the authors, (4) English language articles, (5) studies published by June, 2015, (6) the primary outcome measures included SBP, DBP, TC, HDL, LDL, non-HDL, TG, and (7) outcome not selfreported. The search strategy included (1) electronic searches in multiple databases (PubMed (MEDLINE), Web of Science, and Scopus) on June 5, 2015, and (2) cross-referencing from the reference lists of all retrieved articles (citation tracking). The summaries of the key findings from all three studies are discussed below.

\section{Birth Weight and CVD Risk Factors}

The aim of the first study was to investigate the association between infant's birth weight and childhood CVD risk factors (SBP, DBP, TC, LDL, HDL, non-HDL, and TG) at 11 years of age (controlling for child's current BMI), and the subsequent risk of developing maternal CVD risk factors (TC, LDL, HDL, non-HDL, and TG) 11 years post partum in infants born full-term only.

The final study sample after excluding infants born pre-term (i.e., $<37$ weeks of gestation: $\mathrm{N}=2,097,9.67 \%$ ) was 19,583 . In this sample, nearly $3 \%$ of the infants were low birth weight (LBW) and 11\% were high birth weight (HBW). Whereas current literature on the association between birth weight and CVD risk factors shows inconsistent results, the results of this current study were generally consistent with the Barker's hypothesis for all outcomes except for DBP. The results showed a linear inverse association between birth weight and SBP, TC, LDL, nonHDL, and TG, and a positive association with HDL at 11 years of age after adjusting for child BMI and additional covariates. However, the associations were not statistically significant for SBP and TC in the adjusted models. Although the direction of the association between SBP and birth weight was consistent with previous studies, ${ }^{1-5}$ unlike most studies the adjusted association was not significant $(b=-0.02 \mathrm{mmHg} ; 95 \% \mathrm{CI}:-0.06,0.03 ; \mathrm{p}=0.5295$ for $1,000 \mathrm{~g}$ increase in birth weight). The findings suggest that the negative association between birth weight and SPB is most likely not independent of the child's current BMI. The direction of the association between birth weight and DBP was not consistent with the Barker's hypothesis for both the crude and the adjusted analysis $(b=0.01 \mathrm{mmHg} ; 95 \% \mathrm{CI}$ : $-0.02,0.04 ; \mathrm{p}=0.4913$ for $1,000 \mathrm{~g}$ increase in birth weight). However, the results for DBP are consistent with other studies that also found a significant positive association between birth weight and childhood DBP for the unadjusted analysis ${ }^{6,7}$ that became non-significant after adjusting for the child's current BMI. ${ }^{8}$ Our results found a significant positive association between birth weight and childhood HDL $(b=0.06$ $\mathrm{mg} / \mathrm{dL} ; 95 \% \mathrm{CI}: 0.02,0.09 ; \mathrm{p}=0.0035$ for $1,000 \mathrm{~g}$ increases in birth weight), and a negative association with TG levels $(b=-0.003 \mathrm{mg} / \mathrm{dL} ; 95 \% \mathrm{CI}:-0.05,-0.001 ; \mathrm{p}=0.0005$ for $1,000 \mathrm{~g}$ 
increases in birth weight) which is are also consistent with other studies for $\mathrm{HDL}^{2,9}$ and TG levels respectively. ${ }^{10}$ When adjusted for the child's current BMI the direction of association for HDL (b $=0.14 \mathrm{mg} / \mathrm{dL} ; 95 \% \mathrm{CI}: 0.11,0.18 ; \mathrm{p}<0.0001$ for $1,000 \mathrm{~g}$ increases in birth weight) and log-TG (b $=-0.007 ; 95 \%$ CI: $-0.008,-0.005 ; \mathrm{p}<0.0001$ for $1,000 \mathrm{~g}$ increases in birth weight) remained the same and the magnitude of the association increased. LDL and non-HDL were not significant in the unadjusted analysis but became significant and negatively associated with birth weight after accounting for the child's BMI and additional covariates [LDL $(b=-0.1 \mathrm{mg} / \mathrm{dL} ; 95 \% \mathrm{CI}:-0.19$, $0.16 ; \mathrm{p}=0.0196$ for $1,000 \mathrm{~g}$ increases in birth weight; and for non-HDL $(\mathrm{b}=-0.18 \mathrm{mg} / \mathrm{dL} ; 95 \%$ CI: $-0.28,-0.09 ; p=0.0002$ for $1,000 \mathrm{~g}$ increases in birth weight)], consistent with the findings of other studies. ${ }^{2,5}$

The results from the current study are consistent with the Barker's hypothesis, as LBW is associated with higher LDL, non-HDL, and TGs and lower mean HDL levels independent of the child's current BMI and additional covariates. The results of the sensitivity analysis using spline regression model further verified that the association between birth weight and lipid levels was linear in our population and not U-shaped as demonstrated by some studies. ${ }^{10-12}$ Based on these results, we hypothesize that the association between birth weight and lipids is independent of the child's current BMI levels. Although the observed effect of birth weight as a distal risk factor for the child's lipid levels was small but significant during childhood, this can have a potential amplified impact as these children enter adulthood.

The association between birth weight and maternal CVD risk factors is an active area of research. Only one study conducted in India that examined the relationship between birth weight and maternal lipid levels 8 years post partum found a significant positive association for maternal TG levels only, after adjusting for additional covariates including maternal BMI. ${ }^{13}$ Our study found no significant mean differences in the any of the lipid levels of mothers by birth weight categories of the infant. However, the unadjusted association between birth weight and maternal TC was significant and positive but became non-significant when adjusted for additional covariates in the regression analysis. None of the other lipid levels were significant in either the unadjusted or the adjusted models. However, the direction of association in our study suggests that mothers who give birth to HBW infants seemed to demonstrate a trend towards poor maternal lipid levels 11 years post-partum and is consistent with the previous study. ${ }^{13}$ Whether maternal lipid levels are associated with giving birth to LBW or HBW infants, or whether the birth weight of the infant is a potential risk factor for maternal lipid level in later years, or whether the mother's own birth weight impacted their lipid profile is difficult to establish at this point. The research on this topic is still in its infancy and needs further exploration. 


\section{Breastfeeding and CVD Risk Factors}

The aim of the second study was to investigate the association between infants who were breastfed during infancy and childhood CVD risk factors (SBP, DBP, TC, LDL, HDL, non-HDL, and TG) at 11 years of age (controlling for child's current BMI), and the subsequent risk of developing maternal CVD risk factors (TC, LDL, HDL, non-HDL, and TG) 11 years post partum.

The final study sample after excluding infants born pre-term (i.e. $<37$ weeks of gestation: $\mathrm{N}=1,190,10.2 \%$ ) was 10,457 . Nearly $43 \%$ of the mothers self-reported that they breastfed their index child during infancy. The results showed that children whose mother reported to have breastfed compared to children who were not breastfed in infancy had significantly lower SBP (b $=-1.39 \mathrm{mmHg} ; 95 \% \mathrm{CI}:-1.97,-0.81 ; \mathrm{p}<0.0001)$ and DBP $(\mathrm{b}=-0.79 \mathrm{mmHg} ; 95 \% \mathrm{CI}:-1.26$, $0.33 ; \mathrm{p}=0.0009)$ in unadjusted models. However, adjustments for the child's current BMI attenuated the association between report of breastfeeding and DBP $(b=-0.44 \mathrm{mmHg}$; $95 \% \mathrm{CI}$ : $0.89,0.02 ; \mathrm{p}=0.06)$. Adjustment for the child's current BMI also slightly attenuated the association of breastfeeding to SBP $(b=-0.77 \mathrm{mmHg} ; 95 \% \mathrm{CI}:-1.32,-0.23 ; \mathrm{p}=0.005)$, but adjustments for additional socio-demographic variables eliminated the association of breastfeeding to SBP $(b=-0.43 \mathrm{mmHg} ; 95 \% \mathrm{CI}:-0.98,-0.13 ; \mathrm{p}=0.1349)$. The results of the current study for both SBP and DBP are consistent with the evidence provided by the most recent systematic review with meta-analysis published in $2015 .{ }^{14}$ Horta and colleagues demonstrated that SBP and DBP were lower but not significant in children and adolescents aged 10-19 years who had been breastfed [SBP mean difference $=-1.03$ (95\%CI: -2.07; 0.02) and DBP mean difference $=-0.1(95 \% \mathrm{CI}:-0.65 ; 0.45)]$. Our study further demonstrated that the child's current BMI is a potential mediator for the association between breastfeeding and childhood blood pressure. Breastfeeding has shown to be protective for childhood obesity as demonstrated by several studies, ${ }^{14-19}$ including earlier work with WV fifth grade children. ${ }^{20}$ The results of this current study are also consistent with individual studies as well as with systemic reviews and meta-analyses that found no association between infants who were breastfed vs. non-breastfed and TC, ${ }^{14,21,22}$ LDL, ${ }^{22}$ and non-HDL levels in childhood. However, the results of this current study showed that children whose mother reported to have breastfed compared to children who were not breastfed in infancy had significantly lower TG. The regression coefficient of the unadjusted association was -0.07 (95\% CI: $-0.1,-0.05 ; \mathrm{p}<0.0001)$. Adjustment for the child's current BMI in fifth grade attenuated the association of reported history of breastfeeding to $\mathrm{TG}(\mathrm{b}=-0.05 ; 95 \%$ CI: -0.07, -0.03; $\mathrm{p}=0.048$ ), which further diminished in magnitude but remained significant after adjusting for additional covariates as well $(b=-0.04 ; 95 \% \mathrm{CI}:-0.06,-0.01 ; \mathrm{p}=0.01)$. The final model for TG included, the child's BMI percentile in fifth grade, age, sex, race, maternal 
education at birth, number of previous pregnancies, and family history of cholesterol. However, previous research on this association remains inconclusive with one study finding a significant association only among boys only ${ }^{23}$ or no association at all. ${ }^{24,25}$ This study did not find any differences by gender for the association between breastfeeding and childhood TG levels. Based on the results for TGs, we hypothesize that this association is independent of the child's current BMI. Although the associations were partially attenuated by childhood BMI but remained significant suggests that childhood BMI may be a potential partial mediator. Additionally the unique amount of variance shared between reported history of breastfeeding and TG levels of fifth grade WV children was less than $0.2 \%$, suggesting a small effect size.

The association between maternal history of breastfeeding their infants and maternal CVD risk factors is also an active area of research. Our results are not consistent with studies that found a protective association between breastfeeding and maternal lipid profile. ${ }^{26-28}$ However, it is important to note that for the current study the direction of association was positive for TC, TG, non-HDL, and LDL and negative for HDL, which is consistent with the hypothesis that breastfeeding is protective for maternal cardiovascular health. Some of the potential reasons for the contrasting results could be lack of information on lactation duration or lifetime duration of lactation, which is the basis of most previous research. ${ }^{26,27,29}$ Furthermore, the exposure was assessed retrospectively via parental recall and is subject to self-report bias. It is also possible that most mothers who initiated breastfeeding, failed to continue to breastfeed for longer durations. Additionally, the sample size for the maternal outcomes was small due to very low participation rates. However, Stuebe and colleagues suggest that currently there is no study that has collected comprehensive data to explain the apparent association between breastfeeding and maternal CVD risk factors. ${ }^{30}$ Thus, future studies are needed to collect longitudinal data with wide-ranging information on several socio-demographics, genetic and lifestyle factors in order to examine and understand these associations in mothers.

\section{Childhood Obesity and Adult CVD Risk Factors}

The purpose of the third study was to conduct a comprehensive systematic review (SR) and meta-analysis (MA) to critically evaluate the available evidence regarding the relationship between childhood obesity and adult CVD risk factors (SBP, DBP, TC, LDL, HDL, non-HDL, and TG).

The final number of studies included in the SR was $23^{31-53}$ and 21 studies were included in the MA. ${ }^{31-37,39-47,49-53}$ The findings suggest that childhood obesity is significantly and positively associated with adult SBP $(\mathrm{Zr}=0.11 ; 95 \% \mathrm{CI}, 0.07,0.14), \mathrm{DBP}(\mathrm{Zr}=0.11 ; 95 \% \mathrm{CI}, 0.07,0.14)$, 
and TG $(\mathrm{Zr}=0.08 ; 95 \% \mathrm{CI}, 0.03,0.13)$, and significantly and inversely associated with adult HDL $(\mathrm{Zr}=-0.06 ; 95 \% \mathrm{CI},-0.10,-0.02)$. However, the pooled results of studies that adjusted for adult $\mathrm{BMI}^{32,35,42,44,46,53}$ showed that these associations were significant but negative for SBP $(\mathrm{Zr}=$ $-0.13 ; 95 \% \mathrm{CI},-0.07,-0.14)$, DBP ( $\mathrm{Zr}=-0.11 ; 95 \% \mathrm{CI},-0.17,-0.04)$, and $\mathrm{LDL}(\mathrm{Zr}=-0.08 ; 95 \%$ CI, $-0.12,-0.05)$. Furthermore, the associations between childhood adiposity measure and adult HDL and TG became non-significant when pooling studies that adjusted for adult BMI. However, it is important to point out that less than one third of studies adjusted for adult adiposity measures.

The results of our study are consistent with two systematic reviews by Lloyd and colleagues. ${ }^{54,55}$ Lloyd and colleagues found little evidence that childhood obesity is an independent risk factor for adult blood pressure ${ }^{55}$ and adult lipid levels. ${ }^{54}$ The SR by Park and colleagues ${ }^{56}$ and by Reilly and colleagues,${ }^{57}$ which focused on hypertension and not individual components of hypertension (i.e. SBP and DBP), also found a significant and positive association between childhood adiposity and adult hypertension. However, two out of five studies that adjusted for adult BMI found no association. ${ }^{56}$ The results from the random effects MA by Juonala and colleagues using data from four cohorts showed a significant association between childhood obesity and adult hypertension, LDL, high risk HDL, and TG. When the study adjusted for adult obesity, hypertension remained significant but the effect of childhood adiposity was reduced and became non-significant for dyslipidemias. ${ }^{58}$ The direction of effect for the association between childhood obesity and adult CVD risk factors in the current MA is consistent with the meta-analytic work by Juonala et al..$^{58}$

The results also demonstrated that more rigorous science is needed. For example, the risk of bias assessment was performed using the 'Strengthening the Reporting of Observational studies in Epidemiology' (STROBE) instrument. The results showed that at the study level, nine studies had more than one third of items that were at an increased risk of bias. ${ }^{31,33,38,39,41-43,49,53}$ Other differences between studies may be driven by geographic, gender, or time related covariates. For example, results from the meta-regression analysis revealed that the association between childhood obesity and adult SBP and DBP increases as the baseline age increases. For SBP, DBP, TC, HDL, and LDL the association decreases as the follow-up age and length of follow-up increases. For TC and HDL (for studies that adjusted for adult BMI) the association was lower in males compared to females. For SBP only, the association was higher in United States compared to other countries.

To the best of the investigative team's knowledge, this is the first MA to examine the association between childhood obesity and adult CVD risk factors as well as examine this association in studies that adjusted for adult BMI. The results of the current MA suggest that 
childhood obesity is significantly and positively associated with adult SBP, DBP, and TG and negatively associated with adult HDL. However, additional, well-designed, longitudinal studies are needed before any definitive conclusions can be reached.

\section{Limitations of the Dissertation}

Some of the limitations of the first two studies include lack of information on potential factors that are known to influence the relationship between perinatal exposures and childhood and maternal CVD risk factors. These include but are not limited to: parental adiposity status, maternal pre-pregnancy weight, maternal lipid status at before and during pregnancy, maternal birth weight, rapid weight gain during the first year of life, pubertal status of the child, family history of hypertension, physical activity, and dietary behaviors. Secondly, maternal CVD risk factors were limited to lipid profile only, which was available for a small sample of the population thus limiting the generalizability of the sample. Although the childhood data can be generalizable to WV children, the results may not be generalizable beyond the participants included in this study due to the unique population characteristics of this state. Lastly, for the second study information on actual breastfeeding was obtained retrospectively via parental recall and did not inquire about the method, extent, or the lifetime duration of maternal lactation.

Some of the main limitations of the third study included having a small sample size for most of the outcomes assessed. Due to the small number of included studies we were not able to assess for small-study effects (e.g. potential publication bias) for all the lipid outcomes, and were perhaps underpowered to find a true effect for many of the analyses. Some of the pre-planned analyses to identify sources of heterogeneity were not performed due to the small sample size as well. Lastly, less than one third of the included studies adjusted for adult BMI, which further decreased the sample size of the pooled analysis for the outcomes that examined the relationship between childhood obesity and adult CVD risk factors adjusting for adult adiposity.

\section{Strengths of the Dissertation}

One of the main strengths of the first two studies included using a large longitudinal dataset with information available for important covariates. To our knowledge this is the first study that has examined these associations in Appalachia, as an initial step to reduce the CVD burden by identifying the linkages between perinatal risk factors and later CVD risk factors. Other strengths include the use of directly measured distal exposure (birth weight) and all study outcomes (i.e., they were not based on recall or self-report). Furthermore, the effect size of breastfeeding and childhood TGs levels is a novel finding in 11-year-old children as earlier 
studies included younger or older adolescent populations. ${ }^{23-25,59}$ For the third study, to the best of our knowledge this is the first MA to examine the association between childhood obesity and adult CVD risk factors as well as examine this association in studies that adjusted for adult BMI. Earlier SRs ${ }^{54-57}$ on the topic included studies published up to 2011, thus this SR provides a more updated review of studies examining this association in studies published up to 2015. The current SR also included any definition or classification used in individual studies to measure childhood adiposity, which facilitated in identification of additional eligible studies that examined these associations.

\section{Potential Public Health Implications and Future Recommendations}

CVD is the leading cause of death globally, nationally as well in the state of WV. ${ }^{60-62}$ According to the 2016 Heart Disease and Stroke Statistics update, more than one third of American adults have $\geq 1$ types of $\mathrm{CVD}^{62}$ and it is projected that $43.9 \%$ of the US population will have some form of CVD by $2030 .{ }^{62}$ For 2011-12, the estimated cost of CVD (direct and indirect) was $\$ 316.6$ billion, which is also projected to increase to $\$ 918$ billion by $2030 .{ }^{62}$ High blood pressure and poor cholesterol are well-established risk factors for CVD. ${ }^{63-67}$ Hypertension in children is linked to end-organ damage and poor cholesterol is associated with preclinical atherosclerosis during childhood. ${ }^{68-71}$ These risk factors also track from childhood into adulthood. ${ }^{49,72-78}$ Childhood adiposity is another risk factor for developing CVD risk factors ${ }^{35,79-81}$ and obese children have a higher risk of becoming overweight/obese adults, ${ }^{82-84}$ which is an independent predictor of adult CVD and its risk factors. ${ }^{85-89}$ Over weight and obesity is prevalent in one third of the youth in the U.S. and nearly two third of the adult population. ${ }^{90}$ Research shows that early prevention of CVD risk factors such as obesity, high blood pressure, and cholesterol levels can reduce the risk of CVD in subsequent years. ${ }^{64,91}$

The current set of studies was able to explore the association between perinatal factors (birth weight and breastfeeding) and childhood and maternal CVD risk factors in the state of WV. This rural Appalachian population has one of the highest prevalence of CVD and CVD risk factors in the nation. Thus, there is vital need to first and foremost identify these determinants and their long-term cardiovascular impact in this state as a first step geared towards health promotion and implementation of early preventative interventions for reducing the burden of CVD.

The findings revealed that perinatal exposures such as LBW in full-term infants was associated with worse lipid levels, and breastfeeding as a protective early life exposure was associated with lower lipid levels during childhood. Childhood BMI was a potential mediator between perinatal factors (birth weight and breastfeeding) and childhood CVD risk factors. 
Though the association between perinatal factors as the distal determinant of CVD risk factors was small, these risk factors have the ability to track and potentially amplify, as these children become adults. The results of the study can guide future research to identify early life exposures that may potentially restrict fetal growth resulting in LBW babies in full-term infants in this state. Moreover, biomedical research should focus on identifying complex mechanisms, which underlie the physiological changes in infants who were not breastfed or who were born with LBW that are responsible for less than optimal cardiovascular health in later life. The results from this study can guide future interventions to create targeted prevention or screening programs for children who are born LBW for less than optimal levels of lipids and lipoproteins. Considering the role of BMI as a potential mediator the study can also serve a guide to targeted screening interventions for children who are born LBW and are overweight or obese during childhood for high blood pressure and less than optimal levels of lipids and lipoproteins. Furthermore, future research should focus on establishing different birth cut-offs for diverse populations to increase the sensitivity of identifying infants with higher risk of future CVD as suggested by a recent systematic review as well. ${ }^{92}$ The observed impact of breastfeeding as a distal protective factor for the child's CVD risk factors was small; nonetheless it adds to the argument of promoting breastfeeding practices in this rural Appalachian state of WV. For the maternal CVD risk factors, the current study demonstrates some important preliminary results. The direction of association in the first study suggested that mothers who give birth to HBW babies have higher risk of poor lipid levels and for the second study the direction of correlations suggested breastfeeding to be protective for maternal lipid levels 11 years post-partum. Thus, providing an essential contribution for expanding the research examining the associations between perinatal factors and maternal CVD health in subsequent years.

The results of the current SR with MA have several implications as well. Some of the main implications for research include recommendation for future longitudinal studies to improve their reporting with respect to several potential sources of bias. Additionally, it is recommended that future studies examining the association between childhood obesity and adult CVD risk factors to also report their findings for non-HDL level. This is important because non-HDL has been shown to be better marker of risk for CVD compared to LDL. ${ }^{93,94}$ Moreover, less than one third of the studies included in the SR adjusted for adult adiposity. It is suggested that future studies collect this information and present both crude and adjusted associations. The inverse associations in the adjusted analysis provides the basis for future research to explore whether children at the lower end of the BMI are at a higher risk for developing CVD risk factors compared to children at the higher end of the BMI spectrum during childhood after adjusting for 
adult BMI. Furthermore, this finding adds to the argument that perhaps obese children who become normal weight adults are probably not at any higher risk of CVD risk factor development if they become non-obese in adulthood. However, future robust studies are needed to confirm these results in diverse populations. Overall childhood obesity was a risk factor for select adult CVD risk factors (SBP, DBP, HDL, and TG). These findings can guide future interventions to create screening programs for high blood pressure and less than optimal levels of lipids and lipoproteins in adults who were overweight or obese during childhood and adolescence. Prevention of childhood obesity should remain a priority for public health interventions for preventing negative health outcomes during childhood as well as reducing the burden of adult obesity.

\section{Conclusion}

The main findings of the study demonstrated that LBW is a risk factor for poor lipid levels in childhood (LDL, HDL, non-HDL, and TG) and breastfeeding is a protective factor for childhood lipid level (TG) in fifth grade West Virginian children born full term. No significant associations were observed for perinatal factors (birth weight and breastfeeding) and maternal lipid profile. Well-designed longitudinal studies are needed to understand the complex pathways examining these associations at different life stages. The results of the systematic review with meta-analysis suggest that childhood obesity is significantly and positively associated with adult SBP, DBP, and TG and negatively associated with adult HDL. However, additional, well-designed longitudinal studies with improved reporting as well as data on both unadjusted and adjusted associations for adult adiposity are needed before any definitive conclusions can be reached.

\section{References}




\section{Chapter 1}

1. Roth GA, Forouzanfar MH, Moran AE, et al. Demographic and epidemiologic drivers of global cardiovascular mortality. The New England journal of medicine 2015;372:1333-41. 2. Naghavi M, and Collaborators (712). GBD 2013 Mortality and Causes of Death Collaborators. Global, regional, and national age-sex specific all-cause and cause-specific mortality for 240 causes of death, 1990-2013: a systematic analysis for the Global Burden of Disease Study 2013. Lancet 2015;385:117-71.

3. World Health Organization. Global status report on noncommunicable diseases 2014. Geneva: WHO. Available at: http://www.who.int/mediacentre/factsheets/fs355/en/. 2015.

4. Mozaffarian D, Benjamin EJ, Go AS, et al. Heart Disease and Stroke Statistics-2016 Update: A Report From the American Heart Association. Circulation 2016;133:e38-e360.

5. Mozaffarian D, Benjamin EJ, Go AS, et al. Heart disease and stroke statistics--2015 update: a report from the American Heart Association. Circulation 2015;131:e29-322.

6. Lloyd-Jones D, Adams RJ, Brown TM, et al. Executive summary: heart disease and stroke statistics--2010 update: a report from the American Heart Association. Circulation 2010;121:94854.

7. Roger VL, Go AS, Lloyd-Jones DM, et al. Executive summary: heart disease and stroke statistics--2012 update: a report from the American Heart Association. Circulation 2012;125:18897.

8. Go AS, Mozaffarian D, Roger VL, et al. Executive summary: heart disease and stroke statistics--2014 update: a report from the American Heart Association. Circulation 2014;129:399410.

9. Mudd-Martin G, Biddle MJ, Chung ML, et al. Rural Appalachian perspectives on heart health: social ecological contexts. American journal of health behavior 2014;38:134-43.

10. Ezzati M, Oza S, Danaei G, Murray CJ. Trends and cardiovascular mortality effects of statelevel blood pressure and uncontrolled hypertension in the United States. Circulation 2008; 117:905-14.

11. Halverson JA, Barnett E, Casper M. Geographic disparities in heart disease and stroke mortality among black and white populations in the Appalachian region. Ethnicity \& disease 2002;12:S3-82-91.

12. Cardiovascular disease - Risk factors. Available at http://www.nhs.uk/Conditions/cardiovascular-disease/Pages/Risk-factors.aspx. 2014. (Accessed Feb 13, 2015, 13. Meyer PA, Yoon PW, Kaufmann RB, Centers for Disease C, Prevention. Introduction: CDC Health Disparities and Inequalities Report - United States, 2013. Morbidity and mortality weekly report Surveillance summaries 2013;62 Suppl 3:3-5.

14. O'Donnell MJ, Xavier D, Liu L, et al. Risk factors for ischaemic and intracerebral haemorrhagic stroke in 22 countries (the INTERSTROKE study): a case-control study. Lancet 2010;376:112-23.

15. Yusuf S, Hawken S, Ounpuu S, et al. Effect of potentially modifiable risk factors associated with myocardial infarction in 52 countries (the INTERHEART study): case-control study. Lancet 2004;364:937-52.

16. Stary HC, Blankenhorn DH, Chandler AB, et al. A definition of the intima of human arteries and of its atherosclerosis-prone regions. A report from the Committee on Vascular Lesions of the Council on Arteriosclerosis, American Heart Association. Arteriosclerosis and thrombosis : a journal of vascular biology / American Heart Association 1992;12:120-34.

17. McGill HC, Jr. The pathogenesis of atherosclerosis. Clinical chemistry 1988;34:B33-9. 
18. Bao W, Srinivasan SR, Wattigney WA, Berenson GS. Persistence of multiple cardiovascular risk clustering related to syndrome $\mathrm{X}$ from childhood to young adulthood. The Bogalusa Heart Study. Archives of internal medicine 1994;154:1842-7.

19. Urbina EM, Williams RV, Alpert BS, et al. Noninvasive assessment of subclinical atherosclerosis in children and adolescents: recommendations for standard assessment for clinical research: a scientific statement from the American Heart Association. Hypertension 2009;54:919 50 .

20. Antman E. Policy Report. American Heart Association. Available at http://www.heart.org/idc/groups/heartpublic/@wcm/@adv/documents/downloadable/ucm_478018.pdf

21. Flynn JT, Daniels SR, Hayman LL, et al. Update: ambulatory blood pressure monitoring in children and adolescents: a scientific statement from the American Heart Association. Hypertension 2014;63:1116-35.

22. Ong KL, Tso AW, Lam KS, Cheung BM. Gender difference in blood pressure control and cardiovascular risk factors in Americans with diagnosed hypertension. Hypertension 2008;51:1142-8.

23. Schillaci G, Pirro M, Mannarino E. Assessing cardiovascular risk: should we discard diastolic blood pressure? Circulation 2009;119:210-2.

24. Tin LL, Beevers DG, Lip GY. Systolic vs diastolic blood pressure and the burden of hypertension. Journal of human hypertension 2002;16:147-50.

25. Lawes CM, Vander Hoorn S, Law MR, Elliott P, MacMahon S, Rodgers A. Blood pressure and the global burden of disease 2000. Part II: estimates of attributable burden. Journal of hypertension 2006;24:423-30.

26. Chockalingam A, Campbell NR, Fodor JG. Worldwide epidemic of hypertension. The Canadian journal of cardiology 2006;22:553-5.

27. Kit BK, Kuklina E, Carroll MD, Ostchega Y, Freedman DS, Ogden CL. Prevalence of and trends in dyslipidemia and blood pressure among US children and adolescents, 1999-2012. JAMA pediatrics 2015;169:272-9.

28. Chen X, Wang Y. Tracking of blood pressure from childhood to adulthood: a systematic review and meta-regression analysis. Circulation 2008;117:3171-80.

29. Rosendorff C, Black HR, Cannon CP, et al. Treatment of hypertension in the prevention and management of ischemic heart disease: a scientific statement from the American Heart Association Council for High Blood Pressure Research and the Councils on Clinical Cardiology and Epidemiology and Prevention. Circulation 2007;115:2761-88.

30. Guo X, Zhang X, Guo L, et al. Association between pre-hypertension and cardiovascular outcomes: a systematic review and meta-analysis of prospective studies. Current hypertension reports 2013;15:703-16.

31. Hanevold C, Waller J, Daniels S, Portman R, Sorof J, International Pediatric Hypertension A. The effects of obesity, gender, and ethnic group on left ventricular hypertrophy and geometry in hypertensive children: a collaborative study of the International Pediatric Hypertension Association. Pediatrics 2004;113:328-33.

32. Lande MB, Carson NL, Roy J, Meagher CC. Effects of childhood primary hypertension on carotid intima media thickness: a matched controlled study. Hypertension 2006;48:40-4.

33. Brady TM, Fivush B, Flynn JT, Parekh R. Ability of blood pressure to predict left ventricular hypertrophy in children with primary hypertension. The Journal of pediatrics 2008;152:73-8

34. America's Health Ranking. United Health Foundation. Available at http://www.americashealthrankings.org. 2014. (Accessed March, 06, 2015, 35. The CARDIAC Project 1998-2016. Coronary Artery Risk Detection in Appalachian Communities. Avaiable at http://www.cardiacwv.org. 2016. (Accessed Feburary 12, 2016, 
36. Pischon T, Girman CJ, Sacks FM, Rifai N, Stampfer MJ, Rimm EB. Non-high-density lipoprotein cholesterol and apolipoprotein B in the prediction of coronary heart disease in men. Circulation 2005;112:3375-83.

37. Cholesterol Facts. National Center for Chronic Disease Prevention and Health Promotion, Division for Heart Disease and Stroke Prevention. Available http://www.cdc.gov/cholesterol/facts.htm. 2012. (Accessed Feb 13, 2015, 2015, 38. McGill HC, Jr., McMahan CA, Malcom GT, Oalmann MC, Strong JP. Effects of serum lipoproteins and smoking on atherosclerosis in young men and women. The PDAY Research Group. Pathobiological Determinants of Atherosclerosis in Youth. Arteriosclerosis, thrombosis, and vascular biology 1997;17:95-106.

39. Berenson GS, Srinivasan SR, Bao W, Newman WP, 3rd, Tracy RE, Wattigney WA. Association between multiple cardiovascular risk factors and atherosclerosis in children and young adults. The Bogalusa Heart Study. The New England journal of medicine 1998;338:16506.

40. Davis PH, Dawson JD, Riley WA, Lauer RM. Carotid intimal-medial thickness is related to cardiovascular risk factors measured from childhood through middle age: The Muscatine Study. Circulation 2001;104:2815-9.

41. Li S, Chen W, Srinivasan SR, et al. Childhood cardiovascular risk factors and carotid vascular changes in adulthood: the Bogalusa Heart Study. Jama 2003;290:2271-6.

42. Daniels SR, Greer FR, Committee on N. Lipid screening and cardiovascular health in childhood. Pediatrics 2008;122:198-208.

43. Expert Panel on Integrated Guidelines for Cardiovascular H, Risk Reduction in C, Adolescents, National Heart L, Blood I. Expert panel on integrated guidelines for cardiovascular health and risk reduction in children and adolescents: summary report. Pediatrics 2011;128 Suppl 5:S213-56.

44. Kavey RE, Daniels SR, Lauer RM, et al. American Heart Association guidelines for primary prevention of atherosclerotic cardiovascular disease beginning in childhood. The Journal of pediatrics 2003;142:368-72.

45. National Cholesterol Education Program Expert Panel on Detection E, Treatment of High Blood Cholesterol in A. Third Report of the National Cholesterol Education Program (NCEP) Expert Panel on Detection, Evaluation, and Treatment of High Blood Cholesterol in Adults (Adult Treatment Panel III) final report. Circulation 2002;106:3143-421.

46. ATP III At-A-Glance: Quick Desk Reference. National Institutes of Health. National Heart, Lung, and Blood Institute. NIH Publication No. 01-3305. Avaiable at:

http://www.nhlbi.nih.gov/health-pro/guidelines/current/cholesterol-guidelines/quick-deskreference-html. 2001. (Accessed Feburary 12, 2016, 47. Carroll MD, Kit BK, Lacher DA, Yoon SS. Total and high-density lipoprotein cholesterol in adults: National Health and Nutrition Examination Survey, 2011-2012. NCHS data brief 2013:18.

48. Porkka KV, Viikari JS, Taimela S, Dahl M, Akerblom HK. Tracking and predictiveness of serum lipid and lipoprotein measurements in childhood: a 12-year follow-up. The Cardiovascular Risk in Young Finns study. American journal of epidemiology 1994;140:1096-110.

49. Webber LS, Srinivasan SR, Wattigney WA, Berenson GS. Tracking of serum lipids and lipoproteins from childhood to adulthood. The Bogalusa Heart Study. American journal of epidemiology 1991;133:884-99.

50. Morrison JA, Glueck CJ, Woo JG, Wang P. Risk factors for cardiovascular disease and type 2 diabetes retained from childhood to adulthood predict adult outcomes: the Princeton LRC Followup Study. International journal of pediatric endocrinology 2012;2012:6.

51. Magnussen CG, Raitakari OT, Thomson R, et al. Utility of currently recommended pediatric dyslipidemia classifications in predicting dyslipidemia in adulthood: evidence from the 
Childhood Determinants of Adult Health (CDAH) study, Cardiovascular Risk in Young Finns Study, and Bogalusa Heart Study. Circulation 2008;117:32-42.

52. Poirier P, Giles TD, Bray GA, et al. Obesity and cardiovascular disease: pathophysiology, evaluation, and effect of weight loss. Arteriosclerosis, thrombosis, and vascular biology 2006;26:968-76.

53. Rudolf MC, Krom AJ, Cole TJ. How good are BMI charts for monitoring children's attempts at obesity reduction? Archives of disease in childhood 2012;97:418-22.

54. Stevens J, McClain JE, Truesdale KP. Selection of measures in epidemiologic studies of the consequences of obesity. International journal of obesity 2008;32 Suppl 3:S60-6.

55. Defining Adult Overweight and Obesity. Available at: http://www.cdc.gov/obesity/adult/defining.html. 2012. (Accessed Feburary 12, 2016, 56. Defining Childhood Obesity. BMI for Children and Teens. Division of Nutrition, Physical Activity, and Obesity, National Center for Chronic Disease Prevention and Health Promotion Avaiable at: http://www.cdc.gov/obesity/childhood/defining.html. 2015. (Accessed Feburary 12, 2016,

57. Ogden CL, Carroll MD, Kit BK, Flegal KM. Prevalence of childhood and adult obesity in the United States, 2011-2012. Jama 2014;311:806-14.

58. WVDHHR. Obesity in West Virginia. West Virginia Bureau for Public Health. Department of Health and Human Resources. Available at:

http://www.wvdhhr.org/bph/hsc/pubs/other/obesityreport2011/obesityreport2011.pdf. In:

Resources. WVBfPHDoHaH, ed.2011.

59. 2007 National Survey of Children's Health. Data analysis provided by the Child and

Adolescent Health Measurement Initiative, Data Resource Center.

http://www.childhealthdata.org/. 2007.

60. Serdula MK, Ivery D, Coates RJ, Freedman DS, Williamson DF, Byers T. Do obese children become obese adults? A review of the literature. Preventive medicine 1993;22:167-77.

61. Deshmukh-Taskar P, Nicklas TA, Morales M, Yang SJ, Zakeri I, Berenson GS. Tracking of overweight status from childhood to young adulthood: the Bogalusa Heart Study. European journal of clinical nutrition 2006;60:48-57.

62. Krauss RM, Winston M, Fletcher BJ, Grundy SM. Obesity : impact on cardiovascular disease. Circulation 1998;98:1472-6.

63. Poirier P, Giles TD, Bray GA, et al. Obesity and cardiovascular disease: pathophysiology, evaluation, and effect of weight loss: an update of the 1997 American Heart Association Scientific Statement on Obesity and Heart Disease from the Obesity Committee of the Council on Nutrition, Physical Activity, and Metabolism. Circulation 2006;113:898-918.

64. Eckel RH, Barouch WW, Ershow AG. Report of the National Heart, Lung, and Blood Institute-National Institute of Diabetes and Digestive and Kidney Diseases Working Group on the pathophysiology of obesity-associated cardiovascular disease. Circulation 2002;105:2923-8. 65. Freedman DS, Mei Z, Srinivasan SR, Berenson GS, Dietz WH. Cardiovascular risk factors and excess adiposity among overweight children and adolescents: the Bogalusa Heart Study. J Pediatr 2007;150:12-7 e2.

66. Teixeira PJ, Sardinha LB, Going SB, Lohman TG. Total and regional fat and serum cardiovascular disease risk factors in lean and obese children and adolescents. Obes Res 2001;9:432-42.

67. Celermajer DS, Ayer JG. Childhood risk factors for adult cardiovascular disease and primary prevention in childhood. Heart 2006;92:1701-6.

68. Freedman DS, Khan LK, Dietz WH, Srinivasan SR, Berenson GS. Relationship of childhood obesity to coronary heart disease risk factors in adulthood: the Bogalusa Heart Study. Pediatrics 2001;108:712-8.

69. Bridger T. Childhood obesity and cardiovascular disease. Paediatr Child Health 2009;14:17782. 
70. Friedemann C, Heneghan C, Mahtani K, Thompson M, Perera R, Ward AM. Cardiovascular disease risk in healthy children and its association with body mass index: systematic review and meta-analysis. Bmj 2012;345:e4759.

71. Ford ES, Mokdad AH, Ajani UA. Trends in risk factors for cardiovascular disease among children and adolescents in the United States. Pediatrics 2004;114:1534-44.

72. NIH. Clinical Guidelines on the Identification, Evaluation, and Treatment of Overweight and Obesity in Adults--The Evidence Report. National Institutes of Health. Obes Res 6 Suppl 2:51S209S. 1998.

73. Crowley DI, Khoury PR, Urbina EM, Ippisch HM, Kimball TR. Cardiovascular impact of the pediatric obesity epidemic: higher left ventricular mass is related to higher body mass index. The Journal of pediatrics 2011;158:709-14 e1.

74. Freedman DS, Dietz WH, Srinivasan SR, Berenson GS. The relation of overweight to cardiovascular risk factors among children and adolescents: the Bogalusa Heart Study. Pediatrics 1999; 103:1175-82.

75. Adult Obesity in the United States 2014. The State of Obesity is a project of the Trust for America's Health and the Robert Wood Johnson Foundation. Updated September 21, 2015. http://stateofobesity.org/adult-obesity/. 2015.

76. Zalesin KC, Franklin BA, Miller WM, Peterson ED, McCullough PA. Impact of obesity on cardiovascular disease. The Medical clinics of North America 2011;95:919-37.

77. Must A, Spadano J, Coakley EH, Field AE, Colditz G, Dietz WH. The disease burden associated with overweight and obesity. Jama 1999;282:1523-9.

78. Kotchen TA. Obesity-related hypertension: epidemiology, pathophysiology, and clinical management. American journal of hypertension 2010;23:1170-8.

79. Bogers RP, Bemelmans WJ, Hoogenveen RT, et al. Association of overweight with increased risk of coronary heart disease partly independent of blood pressure and cholesterol levels: a metaanalysis of 21 cohort studies including more than 300000 persons. Archives of internal medicine 2007; 167:1720-8.

80. Brown CD, Higgins M, Donato KA, et al. Body mass index and the prevalence of hypertension and dyslipidemia. Obesity research 2000;8:605-19.

81. USDHHS. The Surgeon General's call

to action to prevent and decrease overweight and obesity. [Rockville, MD]: U.S. Department of Health and Human Services, Public Health Service, Office of the Surgeon General; [2001].

Available from: U.S. GPO, Washington. Available at http://www.surgeongeneral.gov/library. W V Med J 2001;98:234-43.

82. Freedman DS, Dietz WH, Tang R, et al. The relation of obesity throughout life to carotid intima-media thickness in adulthood: the Bogalusa Heart Study. Int J Obes Relat Metab Disord 2004;28:159-66.

83. Baker JL, Olsen LW, Sorensen TI. Childhood body-mass index and the risk of coronary heart disease in adulthood. N Engl J Med 2007;357:2329-37.

84. Lloyd LJ, Langley-Evans SC, McMullen S. Childhood obesity and risk of the adult metabolic syndrome: a systematic review. International journal of obesity 2012;36:1-11.

85. Lloyd LJ, Langley-Evans SC, McMullen S. Childhood obesity and adult cardiovascular disease risk: a systematic review. International journal of obesity 2010;34:18-28.

86. Freedman DS, Khan LK, Serdula MK, Dietz WH, Srinivasan SR, Berenson GS. Interrelationships among childhood BMI, childhood height, and adult obesity: the Bogalusa Heart Study. Int J Obes Relat Metab Disord 2004;28:10-6.

87. Nadeau KJ, Maahs DM, Daniels SR, Eckel RH. Childhood obesity and cardiovascular disease: links and prevention strategies. Nat Rev Cardiol 2011;8:513-25.

88. Sun C, Burgner DP, Ponsonby AL, et al. Effects of early-life environment and epigenetics on cardiovascular disease risk in children: highlighting the role of twin studies. Pediatric research 2013;73:523-30. 
89. Touwslager RN, Houben AJ, Gielen M, et al. Endothelial vasodilatation in newborns is related to body size and maternal hypertension. Journal of hypertension 2012;30:124-31. 90. Holman RL, Mc GH, Jr., Strong JP, Geer JC. The natural history of atherosclerosis: the early aortic lesions as seen in New Orleans in the middle of the of the 20th century. The American journal of pathology 1958;34:209-35.

91. Victora CG, Adair L, Fall C, et al. Maternal and child undernutrition: consequences for adult health and human capital. Lancet 2008;371:340-57.

92. Yu ZB, Han SP, Zhu GZ, et al. Birth weight and subsequent risk of obesity: a systematic review and meta-analysis. Obesity reviews : an official journal of the International Association for the Study of Obesity 2011;12:525-42.

93. Azadbakht L, Kelishadi R, Saraf-Bank S, et al. The association of birth weight with cardiovascular risk factors and mental problems among Iranian school-aged children: the CASPIAN-III study. Nutrition 2014;30:150-8.

94. Bekkers MB, Brunekreef B, Smit HA, et al. Early-life determinants of total and HDL cholesterol concentrations in 8-year-old children; the PIAMA birth cohort study. PloS one 2011;6:e25533.

95. Reynolds D, Hennessy E, Polek E. Is breastfeeding in infancy predictive of child mental wellbeing and protective against obesity at 9 years of age? Child: care, health and development 2014;40:882-90.

96. Lawlor DA, Najman JM, Sterne J, Williams GM, Ebrahim S, Davey Smith G. Associations of parental, birth, and early life characteristics with systolic blood pressure at 5 years of age: findings from the Mater-University study of pregnancy and its outcomes. Circulation 2004;110:2417-23.

97. Martin RM, Ness AR, Gunnell D, Emmett P, Davey Smith G, Team AS. Does breast-feeding in infancy lower blood pressure in childhood? The Avon Longitudinal Study of Parents and Children (ALSPAC). Circulation 2004;109:1259-66.

98. Owen CG, Whincup PH, Kaye SJ, et al. Does initial breastfeeding lead to lower blood cholesterol in adult life? A quantitative review of the evidence. The American journal of clinical nutrition 2008;88:305-14.

99. Barker DJ. The fetal and infant origins of adult disease. Bmj 1990;301:1111.

100. Cooper C, Phillips D, Osmond C, Fall C, Eriksson J. David James Purslove Barker:

clinician, scientist and father of the 'fetal origins hypothesis'. Journal of developmental origins of health and disease 2014;5:161-3.

101. Barker DJ. Fetal origins of coronary heart disease. Bmj 1995;311:171-4.

102. Barker DJ. Fetal origins of coronary heart disease. British heart journal 1993;69:195-6.

103. Skogen JC, Overland S. The fetal origins of adult disease: a narrative review of the epidemiological literature. JRSM short reports 2012;3:59.

104. Filler G, Yasin A, Kesarwani P, Garg AX, Lindsay R, Sharma AP. Big mother or small baby: which predicts hypertension? Journal of clinical hypertension 2011;13:35-41.

105. Gaillard R, Steegers EA, Duijts L, et al. Childhood cardiometabolic outcomes of maternal obesity during pregnancy: the Generation R Study. Hypertension 2014;63:683-91.

106. Nehring I, Lehmann S, von Kries R. Gestational weight gain in accordance to the IOM/NRC criteria and the risk for childhood overweight: a meta-analysis. Pediatric obesity 2013;8:218-24.

107. Rooney BL, Mathiason MA, Schauberger CW. Predictors of obesity in childhood, adolescence, and adulthood in a birth cohort. Maternal and child health journal 2011;15:1166-75. 108. Boney CM, Verma A, Tucker R, Vohr BR. Metabolic syndrome in childhood: association with birth weight, maternal obesity, and gestational diabetes mellitus. Pediatrics 2005;115:e2906.

109. Sanchez J, Priego T, Garcia AP, et al. Maternal supplementation with an excess of different fat sources during pregnancy and lactation differentially affects feeding behavior in offspring: putative role of the leptin system. Molecular nutrition \& food research 2012;56:1715-28. 
110. McMillen IC, Robinson JS. Developmental origins of the metabolic syndrome: prediction, plasticity, and programming. Physiological reviews 2005;85:571-633.

111. Leddy MA, Power ML, Schulkin J. The impact of maternal obesity on maternal and fetal health. Reviews in obstetrics \& gynecology 2008;1:170-8.

112. Fraser A, Tilling K, Macdonald-Wallis C, et al. Associations of gestational weight gain with maternal body mass index, waist circumference, and blood pressure measured $16 \mathrm{y}$ after pregnancy: the Avon Longitudinal Study of Parents and Children (ALSPAC). The American journal of clinical nutrition 2011;93:1285-92.

113. McClure CK, Catov JM, Ness R, Bodnar LM. Associations between gestational weight gain and BMI, abdominal adiposity, and traditional measures of cardiometabolic risk in mothers $8 \mathrm{y}$ postpartum. The American journal of clinical nutrition 2013;98:1218-25.

114. Bukowski R, Davis KE, Wilson PW. Delivery of a small for gestational age infant and greater maternal risk of ischemic heart disease. PloS one 2012;7:e33047.

115. Natland Fagerhaug T, Forsmo S, Jacobsen GW, Midthjell K, Andersen LF, Ivar Lund Nilsen T. A prospective population-based cohort study of lactation and cardiovascular disease mortality: the HUNT study. BMC public health 2013;13:1070.

116. Smith GD, Whitley E, Gissler M, Hemminki E. Birth dimensions of offspring, premature birth, and the mortality of mothers. Lancet 2000;356:2066-7.

117. Smith GD, Sterne J, Tynelius P, Lawlor DA, Rasmussen F. Birth weight of offspring and subsequent cardiovascular mortality of the parents. Epidemiology 2005;16:563-9.

118. Smith GD, Hypponen E, Power C, Lawlor DA. Offspring birth weight and parental mortality: prospective observational study and meta-analysis. American journal of epidemiology 2007;166:160-9.

119. Li CY, Chen HF, Sung FC, et al. Offspring birth weight and parental cardiovascular mortality. International journal of epidemiology 2010;39:1082-90.

120. Smith GC, Pell JP, Walsh D. Pregnancy complications and maternal risk of ischaemic heart disease: a retrospective cohort study of 129,290 births. Lancet 2001;357:2002-6.

121. Catov JM, Lewis CE, Lee M, Wellons MF, Gunderson EP. Preterm birth and future maternal blood pressure, inflammation, and intimal-medial thickness: the CARDIA study. Hypertension 2013;61:641-6.

122. Yajnik CS, Joglekar CV, Pandit AN, et al. Higher offspring birth weight predicts the metabolic syndrome in mothers but not fathers 8 years after delivery: the Pune Children's Study. Diabetes 2003;52:2090-6.

123. Schwarz EB, Ray RM, Stuebe AM, et al. Duration of lactation and risk factors for maternal cardiovascular disease. Obstetrics and gynecology 2009;113:974-82.

124. Krieger N. Proximal, distal, and the politics of causation: what's level got to do with it? American journal of public health 2008;98:221-30.

125. Lammle L, Woll A, Mensink GB, Bos K. Distal and proximal factors of health behaviors and their associations with health in children and adolescents. International journal of environmental research and public health 2013;10:2944-78.

126. Catov JM, Newman AB, Roberts JM, et al. Association between infant birth weight and maternal cardiovascular risk factors in the health, aging, and body composition study. Annals of epidemiology 2007; 17:36-43.

127. Ebina S, Kashiwakura I. Influence of breastfeeding on maternal blood pressure at one month postpartum. International journal of women's health 2012;4:333-9.

128. McClure CK, Catov JM, Ness RB, Schwarz EB. Lactation and maternal subclinical cardiovascular disease among premenopausal women. American journal of obstetrics and gynecology 2012;207:46 e1-8. 


\section{Chapter 2}

1. Roth GA, Forouzanfar MH, Moran AE, et al. Demographic and epidemiologic drivers of global cardiovascular mortality. The New England journal of medicine 2015;372:1333-41. 2. Naghavi M, and Collaborators (712). GBD 2013 Mortality and Causes of Death Collaborators. Global, regional, and national age-sex specific all-cause and cause-specific mortality for 240 causes of death, 1990-2013: a systematic analysis for the Global Burden of Disease Study 2013. Lancet 2015;385:117-71.

3. Mozaffarian D, Benjamin EJ, Go AS, et al. Heart Disease and Stroke Statistics-2016 Update: A Report From the American Heart Association. Circulation 2016;133:e38-e360.

4. Ong KL, Tso AW, Lam KS, Cheung BM. Gender difference in blood pressure control and cardiovascular risk factors in Americans with diagnosed hypertension. Hypertension 2008;51:1142-8.

5. Chobanian AV, Bakris GL, Black HR, et al. Seventh report of the Joint National Committee on Prevention, Detection, Evaluation, and Treatment of High Blood Pressure. Hypertension 2003;42:1206-52.

6. O'Donnell MJ, Xavier D, Liu L, et al. Risk factors for ischaemic and intracerebral haemorrhagic stroke in 22 countries (the INTERSTROKE study): a case-control study. Lancet 2010;376:112-23.

7. Yusuf S, Hawken S, Ounpuu S, et al. Effect of potentially modifiable risk factors associated with myocardial infarction in 52 countries (the INTERHEART study): case-control study. Lancet 2004;364:937-52.

8. Cholesterol Facts. National Center for Chronic Disease Prevention and Health Promotion, Division for Heart Disease and Stroke Prevention. Available http://www.cdc.gov/cholesterol/facts.htm. 2012. (Accessed Feb 13, 2015, 2015, 9. Azadbakht L, Kelishadi R, Saraf-Bank S, et al. The association of birth weight with cardiovascular risk factors and mental problems among Iranian school-aged children: the CASPIAN-III study. Nutrition 2014;30:150-8.

10. Bekkers MB, Brunekreef B, Smit HA, et al. Early-life determinants of total and HDL cholesterol concentrations in 8-year-old children; the PIAMA birth cohort study. PloS one 2011;6:e25533.

11. Institute of Medicine (US) Committee on Preventing the Global Epidemic of Cardiovascular Disease: Meeting the Challenges in Developing Countries; Fuster V, Kelly BB, editors. Promoting Cardiovascular Health in the Developing World: A Critical Challenge to Achieve Global Health. Washington (DC): National Academies Press (US); 2010. 6, Cardiovascular Health Promotion Early in Life. Available from:

http://www.ncbi.nlm.nih.gov/books/NBK45695/. 2010.

12. Kaar JL, Crume T, Brinton JT, Bischoff KJ, McDuffie R, Dabelea D. Maternal obesity, gestational weight gain, and offspring adiposity: the exploring perinatal outcomes among children study. The Journal of pediatrics 2014;165:509-15.

13. Nehring I, Chmitorz A, Reulen H, von Kries R, Ensenauer R. Gestational diabetes predicts the risk of childhood overweight and abdominal circumference independent of maternal obesity. Diabetic medicine : a journal of the British Diabetic Association 2013;30:1449-56.

14. El-Behadli AF, Sharp C, Hughes SO, Obasi EM, Nicklas TA. Maternal depression, stress and feeding styles: towards a framework for theory and research in child obesity. The British journal of nutrition 2015;113 Suppl:S55-71.

15. Laura HC, Menezes AB, Noal RB, Hallal PC, Araujo CL. Maternal anthropometric characteristics in pregnancy and blood pressure among adolescents: 1993 live birth cohort, Pelotas, southern Brazil. BMC public health 2010;10:434. 
16. Fraser A, Tilling K, Macdonald-Wallis C, et al. Association of maternal weight gain in pregnancy with offspring obesity and metabolic and vascular traits in childhood. Circulation 2010;121:2557-64.

17. Owen CG, Whincup PH, Kaye SJ, et al. Does initial breastfeeding lead to lower blood cholesterol in adult life? A quantitative review of the evidence. The American journal of clinical nutrition 2008;88:305-14.

18. Barker DJ, Gluckman PD, Godfrey KM, Harding JE, Owens JA, Robinson JS. Fetal nutrition and cardiovascular disease in adult life. Lancet 1993;341:938-41.

19. Sola-Visner M. Cardiovascular disease and weight ... at birth. Blood 2011;118:1439-41.

20. Bateson P, Barker D, Clutton-Brock T, et al. Developmental plasticity and human health. Nature 2004;430:419-21.

21. World Health Organization, International statistical classification of diseases and related health problems, tenth revision, World Health Organization, Geneva, 1992. 1992.

22. PedNSS Health Indicators. Division of Nutrition, Physical Activity and Obesity, National Center for Chronic Disease Prevention and Health Promotion. Available at http://www.cdc.gov/pednss/pednss_tables/pdf/national_table2.pdf. 2012. (Accessed March, 6, 2015,

23. Pediatric Nutrition Surveillance System (PedNSS) and the Pregnancy Surveillance System (PNSS). Division of Nutrition and Physical Activity. National Center for Chronic Disease

Prevention and Health Promotion. Centers for Disease Control and Prevention. United States Department of Health and Human Services. Available at: http://www.cdc.gov/pednss/index.htm. PDF: http://www.cdc.gov/pednss/pednss_tables/pdf/national_table7.pdf. 2014. (Accessed March 6, 2015, at http://www.cdc.gov/pednss/pednss_tables/pdf/national_table2.pdf.)

24. America's Health Ranking. United Health Foundation. Available at http://www.americashealthrankings.org. 2014. (Accessed March, 06, 2015, 25. Huxley R, Owen CG, Whincup PH, et al. Is birth weight a risk factor for ischemic heart disease in later life? The American journal of clinical nutrition 2007;85:1244-50.

26. de Jong F, Monuteaux MC, van Elburg RM, Gillman MW, Belfort MB. Systematic review and meta-analysis of preterm birth and later systolic blood pressure. Hypertension 2012;59:22634.

27. Risnes KR, Vatten LJ, Baker JL, et al. Birthweight and mortality in adulthood: a systematic review and meta-analysis. International journal of epidemiology 2011;40:647-61.

28. Wilcox AJ. On the importance--and the unimportance--of birthweight. International journal of epidemiology 2001;30:1233-41.

29. Eriksson M, Wallander MA, Krakau I, Wedel H, Svardsudd K. The impact of birth weight on coronary heart disease morbidity and mortality in a birth cohort followed up for 85 years: a population-based study of men born in 1913. Journal of internal medicine 2004;256:472-81. 30. Li CY, Chen HF, Sung FC, et al. Offspring birth weight and parental cardiovascular mortality. International journal of epidemiology 2010;39:1082-90.

31. Bonamy AK, Parikh NI, Cnattingius S, Ludvigsson JF, Ingelsson E. Birth characteristics and subsequent risks of maternal cardiovascular disease: effects of gestational age and fetal growth. Circulation 2011;124:2839-46.

32. Zarrati M, Shidfar F, Razmpoosh E, et al. Does low birth weight predict hypertension and obesity in schoolchildren? Annals of nutrition \& metabolism 2013;63:69-76.

33. Gademan MG, van Eijsden M, Roseboom TJ, van der Post JA, Stronks K, Vrijkotte TG. Maternal prepregnancy body mass index and their children's blood pressure and resting cardiac autonomic balance at age 5 to 6 years. Hypertension 2013;62:641-7.

34. Frontini MG, Srinivasan SR, Xu J, Berenson GS. Low birth weight and longitudinal trends of cardiovascular risk factor variables from childhood to adolescence: the bogalusa heart study.

BMC pediatrics 2004;4:22. 
35. Donker GA, Labarthe DR, Harrist RB, et al. Low birth weight and serum lipid concentrations at age 7-11 years in a biracial sample. American journal of epidemiology 1997;145:398-407. 36. Zhang Z, Kris-Etherton PM, Hartman TJ. Birth weight and risk factors for cardiovascular disease and type 2 diabetes in US children and adolescents: 10 year results from NHANES. Maternal and child health journal 2014;18:1423-32.

37. Filler G, Yasin A, Kesarwani P, Garg AX, Lindsay R, Sharma AP. Big mother or small baby: which predicts hypertension? Journal of clinical hypertension 2011;13:35-41.

38. Sousa MA, Guimaraes IC, Daltro C, Guimaraes AC. Association between birth weight and cardiovascular risk factors in adolescents. Arquivos brasileiros de cardiologia 2013;101:9-17.

39. Hardy R, Sovio U, King VJ, et al. Birthweight and blood pressure in five European birth cohort studies: an investigation of confounding factors. European journal of public health 2006; 16:21-30.

40. Mu M, Wang SF, Sheng J, et al. Birth weight and subsequent blood pressure: a meta-analysis. Archives of cardiovascular diseases 2012;105:99-113.

41. Tilling K, Davies N, Windmeijer F, et al. Is infant weight associated with childhood blood pressure? Analysis of the Promotion of Breastfeeding Intervention Trial (PROBIT) cohort. International journal of epidemiology 2011;40:1227-37.

42. Amorim Rde J, Coelho AF, de Lira PI, Lima Mde C. Is breastfeeding protective for blood pressure in schoolchildren? A cohort study in northeast Brazil. Breastfeeding medicine : the official journal of the Academy of Breastfeeding Medicine 2014;9:149-56.

43. Laor A, Stevenson DK, Shemer J, Gale R, Seidman DS. Size at birth, maternal nutritional status in pregnancy, and blood pressure at age 17: population based analysis. Bmj 1997;315:44953.

44. Malin G, Morris R, Riley R, Teune M, Khan K. When is birthweight at term ( $>/=37$ weeks' gestation) abnormally low? A systematic review and meta-analysis of the prognostic and predictive ability of current birthweight standards for childhood and adult outcomes. BJOG : an international journal of obstetrics and gynaecology 2015.

45. Bergel E, Haelterman E, Belizan J, Villar J, Carroli G. Perinatal factors associated with blood pressure during childhood. American journal of epidemiology 2000;151:594-601.

46. Fenton TR. A new growth chart for preterm babies: Babson and Benda's chart updated with recent data and a new format. BMC pediatrics 2003;3:13.

47. Mullett MD, Cottrell L, Lilly C, et al. Association between birth characteristics and coronary disease risk factors among fifth graders. The Journal of pediatrics 2014;164:78-82.

48. Thorsdottir I, Gunnarsdottir I, Palsson GI. Association of birth weight and breast-feeding with coronary heart disease risk factors at the age of 6 years. Nutrition, metabolism, and cardiovascular diseases : NMCD 2003;13:267-72.

49. Gomes FM, Subramanian SV, Escobar AM, et al. No association between low birth weight and cardiovascular risk factors in early adulthood: evidence from Sao Paulo, Brazil. PloS one 2013;8:e66554.

50. Smith GD, Whitley E, Gissler M, Hemminki E. Birth dimensions of offspring, premature birth, and the mortality of mothers. Lancet 2000;356:2066-7.

51. Smith GD, Sterne J, Tynelius P, Lawlor DA, Rasmussen F. Birth weight of offspring and subsequent cardiovascular mortality of the parents. Epidemiology 2005;16:563-9.

52. Smith GD, Hypponen E, Power C, Lawlor DA. Offspring birth weight and parental mortality: prospective observational study and meta-analysis. American journal of epidemiology 2007;166:160-9.

53. Smith GC, Pell JP, Walsh D. Pregnancy complications and maternal risk of ischaemic heart disease: a retrospective cohort study of 129,290 births. Lancet 2001;357:2002-6.

54. Ngo AD, Roberts CL, Chen JS, Figtree G. Delivery of a Small-For-Gestational-Age Infant and Risk of Maternal Cardiovascular Disease - A Population-Based Record Linkage Study. Heart, lung \& circulation 2015. 
55. Bukowski R, Davis KE, Wilson PW. Delivery of a small for gestational age infant and greater maternal risk of ischemic heart disease. PloS one 2012;7:e33047.

56. Lykke JA, Langhoff-Roos J, Lockwood CJ, Triche EW, Paidas MJ. Mortality of mothers from cardiovascular and non-cardiovascular causes following pregnancy complications in first delivery. Paediatric and perinatal epidemiology 2010;24:323-30.

57. Catov JM, Lewis CE, Lee M, Wellons MF, Gunderson EP. Preterm birth and future maternal blood pressure, inflammation, and intimal-medial thickness: the CARDIA study. Hypertension 2013;61:641-6.

58. Yajnik CS, Joglekar CV, Pandit AN, et al. Higher offspring birth weight predicts the metabolic syndrome in mothers but not fathers 8 years after delivery: the Pune Children's Study. Diabetes 2003;52:2090-6.

59. Xu J, Barinas-Mitchell E, Kuller LH, Youk AO, Catov JM. Maternal hypertension after a low-birth-weight delivery differs by race/ethnicity: evidence from the National Health and Nutrition Examination Survey (NHANES) 1999-2006. PloS one 2014;9:e104149.

60. Lawlor DA, Davey Smith G, Whincup P, et al. Association between offspring birth weight and atherosclerosis in middle aged men and women: British Regional Heart Study. Journal of epidemiology and community health 2003;57:462-3.

61. Behrman RE, Butler, A. S. . Neurodevelopmental, Health, and Family Outcomes for Infants Born Preterm. Preterm Birth: Causes, Consequences, and Prevention. Washington (DC). 2007, National Academy of Sciences.; 2007.

62. Alexander GR. Prematurity at Birth: Determinants, Consequences, and Geographic Variation Preterm Birth: Causes, Consequences, and Prevention. Washington (DC). National Academy of Sciences.; 2007.

63. Hjalmarson O, Sandberg K. Abnormal lung function in healthy preterm infants. American journal of respiratory and critical care medicine 2002;165:83-7.

64. Moreira RS, Magalhaes LC, Alves CR. Effect of preterm birth on motor development, behavior, and school performance of school-age children: a systematic review. Jornal de pediatria 2014;90:119-34.

65. Wilson J. The Barker hypothesis. An analysis. The Australian \& New Zealand journal of obstetrics \& gynaecology 1999;39:1-7.

66. Kramer MS. Determinants of low birth weight: methodological assessment and meta-analysis. Bulletin of the World Health Organization 1987;65:663-737.

67. Delisle H. Programming of chronic disease by impaired fetal nutrition. Evidence and implications for policy and intervention strategies. World Health Organization.2002.

68. Huxley RR, Shiell AW, Law CM. The role of size at birth and postnatal catch-up growth in determining systolic blood pressure: a systematic review of the literature. Journal of hypertension 2000;18:815-31.

69. Huxley R, Owen CG, Whincup PH, Cook DG, Colman S, Collins R. Birth weight and subsequent cholesterol levels: exploration of the "fetal origins" hypothesis. Jama 2004;292:275564.

70. Lawlor DA, Najman JM, Sterne J, Williams GM, Ebrahim S, Davey Smith G. Associations of parental, birth, and early life characteristics with systolic blood pressure at 5 years of age:

findings from the Mater-University study of pregnancy and its outcomes. Circulation 2004;110:2417-23.

71. Murtaugh MA, Jacobs DR, Jr., Moran A, Steinberger J, Sinaiko AR. Relation of birth weight to fasting insulin, insulin resistance, and body size in adolescence. Diabetes care 2003;26:187-92. 72. Schellong K, Schulz S, Harder T, Plagemann A. Birth weight and long-term overweight risk: systematic review and a meta-analysis including 643,902 persons from 66 studies and 26 countries globally. PloS one 2012;7:e47776. 
73. Terry MB, Wei Y, Esserman D, McKeague IW, Susser E. Pre- and postnatal determinants of childhood body size: cohort and sibling analyses. Journal of developmental origins of health and disease 2011;2:99-111.

74. Li N, Strobino D, Ahmed S, Minkovitz CS. Is there a healthy foreign born effect for childhood obesity in the United States? Maternal and child health journal 2011;15:310-23.

75. Geelhoed JJ, Fraser A, Tilling K, et al. Preeclampsia and gestational hypertension are associated with childhood blood pressure independently of family adiposity measures: the Avon Longitudinal Study of Parents and Children. Circulation 2010;122:1192-9.

76. Chiolero A, Paradis G, Kaufman JS. Assessing the possible direct effect of birth weight on childhood blood pressure: a sensitivity analysis. American journal of epidemiology 2014;179:411 .

77. West Virginia Birth Score Project. This program is funded under an agreement with the West Virginia Department of Health and Human Resources, Bureau for Public Health, Office of Maternal, Child and Family Health. Available at http://www.wvdhhr.org/birthscore/. 2013. at http://www.wvdhhr.org/birthscore/.)

78. Myerberg DZ, Carpenter RG, Myerberg CF, Britton CM, Bailey CW, Fink BE. Reducing postneonatal mortality in West Virginia: a statewide intervention program targeting risk identified at and after birth. Am J Public Health 1995;85:631-7.

79. Muratova VN, Demerath EW, Spangler E, et al. The relation of obesity to cardiovascular risk factors among children: the CARDIAC project. W V Med J 2002;98:263-7.

80. Cottrell L, John C, Murphy E, et al. Individual-, family-, community-, and policy-level impact of a school-based cardiovascular risk detection screening program for children in underserved, rural areas: the CARDIAC Project. J Obes 2013;2013:732579.

81. Harris CV, Neal WA. Assessing BMI in West Virginia schools: parent perspectives and the influence of context. Pediatrics 2009;124 Suppl 1:S63-72.

82. Demerath E, Muratova V, Spangler E, Li J, Minor VE, Neal WA. School-based obesity screening in rural Appalachia. Prev Med 2003;37:553-60.

83. Ice CL, Murphy E, Minor VE, Neal WA. Metabolic syndrome in fifth grade children with acanthosis nigricans: results from the CARDIAC project. World journal of pediatrics : WJP 2009;5:23-30.

84. Lauren L, Jarvelin MR, Elliott P, et al. Relationship between birthweight and blood lipid concentrations in later life: evidence from the existing literature. International journal of epidemiology 2003;32:862-76.

85. Must A, Anderson SE. Body mass index in children and adolescents: considerations for population-based applications. International journal of obesity 2006;30:590-4.

86. Kuczmarski RJ, Ogden CL, Guo SS, et al. 2000 CDC Growth Charts for the United States: methods and development. Vital and health statistics Series 11, Data from the national health survey 2002:1-190.

87. U.S. Census Bureau: State and County QuickFacts. Data derived from Population Estimates, American Community Survey, Census of Population and Housing, State and County Housing Unit Estimates, County Business Patterns, Nonemployer Statistics, Economic Census, Survey of Business Owners, Building Permits vol. 2014. 2011.

88. Roberts RJ, Leary SD, Smith GD, Ness AR, Team AS. Maternal age in pregnancy and offspring blood pressure in childhood in the Avon Longitudinal Study of Parents and Children (ALSPAC). Journal of human hypertension 2005;19:893-900.

89. Savage T, Derraik JG, Miles HL, Mouat F, Hofman PL, Cutfield WS. Increasing maternal age is associated with taller stature and reduced abdominal fat in their children. PloS one 2013;8:e58869.

90. Gibbs CM, Wendt A, Peters S, Hogue CJ. The impact of early age at first childbirth on maternal and infant health. Paediatric and perinatal epidemiology 2012;26 Suppl 1:259-84. 
91. Sim JH, Chung D, Lim JS, et al. Maternal age at first delivery is associated with the risk of metabolic syndrome in postmenopausal women: from 2008-2010 Korean National Health and Nutrition Examination Survey. PloS one 2015;10:e127860.

92. Astone NM, Misra D, Lynch C. The effect of maternal socio-economic status throughout the lifespan on infant birthweight. Paediatric and perinatal epidemiology 2007;21:310-8.

93. Reichman NE, Pagnini DL. Maternal age and birth outcomes: data from New Jersey. Family planning perspectives 1997;29:268-72, 95.

94. Gage TB, Fang F, O'Neill E, Dirienzo G. Maternal education, birth weight, and infant mortality in the United States. Demography 2013;50:615-35.

95. Parker JD, Schoendorf KC, Kiely JL. Associations between measures of socioeconomic status and low birth weight, small for gestational age, and premature delivery in the United States.

Annals of epidemiology 1994;4:271-8.

96. Schwartz IL. Low-birth-weight effects of demographic and socioeconomic variables and prenatal care in Pima County, Arizona. The Western journal of medicine 1990;152:725-8.

97. van den Berg G, van Eijsden M, Galindo-Garre F, Vrijkotte TG, Gemke RJ. Explaining socioeconomic inequalities in childhood blood pressure and prehypertension: the ABCD study. Hypertension 2013;61:35-41.

98. Clark AM, DesMeules M, Luo W, Duncan AS, Wielgosz A. Socioeconomic status and cardiovascular disease: risks and implications for care. Nature reviews Cardiology 2009;6:712-

22.

99. Dekkers JC, Treiber FA, Kapuku G, Snieder H. Differential influence of family history of hypertension and premature myocardial infarction on systolic blood pressure and left ventricular mass trajectories in youth. Pediatrics 2003;111:1387-93.

100. Bergstrom E, Hernell O, Persson LA, Vessby B. Serum lipid values in adolescents are related to family history, infant feeding, and physical growth. Atherosclerosis 1995;117:1-13. 101. Gaillard R, Rurangirwa AA, Williams MA, et al. Maternal parity, fetal and childhood growth, and cardiometabolic risk factors. Hypertension 2014;64:266-74.

102. Whincup PH, Cook DG, Papacosta O. Do maternal and intrauterine factors influence blood pressure in childhood? Archives of disease in childhood 1992;67:1423-9.

103. Shah PS, Knowledge Synthesis Group on Determinants of LBWPTb. Parity and low birth weight and preterm birth: a systematic review and meta-analyses. Acta obstetricia et gynecologica Scandinavica 2010;89:862-75.

104. Catov JM, Newman AB, Sutton-Tyrrell K, et al. Parity and cardiovascular disease risk among older women: how do pregnancy complications mediate the association? Annals of epidemiology 2008;18:873-9.

105. Cowan LD, Go OT, Howard BV, et al. Parity, postmenopausal estrogen use, and cardiovascular disease risk factors in American Indian women: the Strong Heart Study. Journal of women's health / the official publication of the Society for the Advancement of Women's Health Research 1997;6:441-9.

106. Ayer JG, Belousova E, Harmer JA, David C, Marks GB, Celermajer DS. Maternal cigarette smoking is associated with reduced high-density lipoprotein cholesterol in healthy 8-year-old children. European heart journal 2011;32:2446-53.

107. Huang RC, Mori TA, Beilin LJ. Early life programming of cardiometabolic disease in the Western Australian pregnancy cohort (Raine) study. Clinical and experimental pharmacology \& physiology 2012;39:973-8.

108. Chiolero A, Bovet P, Paccaud F. Association between maternal smoking and low birth weight in Switzerland: the EDEN study. Swiss medical weekly 2005;135:525-30.

109. Stojanovic M, Bojanic V, Musovic D, et al. [Maternal smoking during pregnancy and socioeconomic factors as predictors of low birth weight in term pregnancies in Nis].

Vojnosanitetski pregled 2010;67:145-50. 
110. Scheers Andersson E, Tynelius P, Nohr EA, Sorensen TI, Rasmussen F. No association of maternal gestational weight gain with offspring blood pressure and hypertension at age 18 years in male sibling-pairs: a prospective register-based cohort study. PloS one 2015;10:e0121202.

111. Mamun AA, O'Callaghan M, Callaway L, Williams G, Najman J, Lawlor DA. Associations of gestational weight gain with offspring body mass index and blood pressure at 21 years of age: evidence from a birth cohort study. Circulation 2009;119:1720-7.

112. Jwa SC, Fujiwara T, Kondo N. Latent protective effects of breastfeeding on late childhood overweight and obesity: a nationwide prospective study. Obesity 2014;22:1527-37.

113. Plagemann A, Harder T. Breast feeding and the risk of obesity and related metabolic diseases in the child. Metabolic Syndrome and Related Disorders 2005;3:222-32.

114. Ip S, Chung M, Raman G, et al. Breastfeeding and maternal and infant health outcomes in developed countries. Evid Rep Technol Assess (Full Rep) 2007:1-186.

115. Toma TS, Rea MF. [Benefits of breastfeeding for maternal and child health: an essay on the scientific evidence]. Cadernos de saude publica 2008;24 Suppl 2:S235-46.

116. Ram KT, Bobby P, Hailpern SM, et al. Duration of lactation is associated with lower prevalence of the metabolic syndrome in midlife--SWAN, the study of women's health across the nation. American journal of obstetrics and gynecology 2008;198:268 e1-6.

117. Umer A, Hamilton C, Britton CM, et al. Association between Breastfeeding and Childhood Obesity: Analysis of a Linked Longitudinal Study of Rural Appalachian Fifth-Grade Children. Childhood obesity 2015;11:449-55.

118. Hurley D, Hullsey, J., McKeown, R., Addy, C. . An Evaluation of Splines in Linear RegressionPaper 147-31. Available at: Chttp://www2.sas.com/proceedings/sugi31/147-31.pdf. 119. Huang N. Scenarios Where Utilizing a Spline Model in Developing a Regression Model Is Appropriate. Available at: http://support.sas.com/resources/papers/proceedings14/1760-2014.pdf. 120. Menezes AM, Hallal PC, Horta BL, et al. Size at birth and blood pressure in early adolescence: a prospective birth cohort study. American journal of epidemiology 2007;165:611-6. 121. Gamborg M, Byberg L, Rasmussen F, et al. Birth weight and systolic blood pressure in adolescence and adulthood: meta-regression analysis of sex- and age-specific results from 20 Nordic studies. American journal of epidemiology 2007;166:634-45.

122. Frese EM, Fick A, Sadowsky HS. Blood pressure measurement guidelines for physical therapists. Cardiopulmonary physical therapy journal 2011;22:5-12.

123. Pickering TG, Hall JE, Appel LJ, et al. Recommendations for blood pressure measurement in humans and experimental animals: part 1: blood pressure measurement in humans: a statement for professionals from the Subcommittee of Professional and Public Education of the American Heart Association Council on High Blood Pressure Research. Circulation 2005;111:697-716. 124. Tin LL, Beevers DG, Lip GY. Systolic vs diastolic blood pressure and the burden of hypertension. Journal of human hypertension 2002;16:147-50.

125. Owen CG, Whincup PH, Odoki K, Gilg JA, Cook DG. Birth weight and blood cholesterol level: a study in adolescents and systematic review. Pediatrics 2003;111:1081-9.

126. Huang RC, Burke V, Newnham JP, et al. Perinatal and childhood origins of cardiovascular disease. International journal of obesity 2007;31:236-44.

127. Haney EM, Huffman LH, Bougatsos C, Freeman M, Steiner RD, Nelson HD. Screening and treatment for lipid disorders in children and adolescents: systematic evidence review for the US Preventive Services Task Force. Pediatrics 2007;120:e189-214.

128. Frerichs RR, Srinivasan SR, Webber LS, Berenson GR. Serum cholesterol and triglyceride levels in 3,446 children from a biracial community: the Bogalusa Heart Study. Circulation 1976;54:302-9.

129. Dai S, Fulton JE, Harrist RB, Grunbaum JA, Steffen LM, Labarthe DR. Blood lipids in children: age-related patterns and association with body-fat indices: Project HeartBeat! American journal of preventive medicine 2009;37:S56-64. 
130. Walter M. Interrelationships among HDL metabolism, aging, and atherosclerosis. Arteriosclerosis, thrombosis, and vascular biology 2009;29:1244-50.

131. Dai S, Yang Q, Yuan K, et al. Non-high-density lipoprotein cholesterol: distribution and prevalence of high serum levels in children and adolescents: United States National Health and Nutrition Examination Surveys, 2005-2010. The Journal of pediatrics 2014;164:247-53.

132. Sumner AE. Ethnic differences in triglyceride levels and high-density lipoprotein lead to underdiagnosis of the metabolic syndrome in black children and adults. The Journal of pediatrics 2009;155:S7 e-11.

133. Expert Panel on Integrated Guidelines for Cardiovascular H, Risk Reduction in C, Adolescents, National Heart L, Blood I. Expert panel on integrated guidelines for cardiovascular health and risk reduction in children and adolescents: summary report. Pediatrics 2011;128 Suppl 5:S213-56.

134. van den Berg G, van Eijsden M, Vrijkotte TG, Gemke RJ. Socioeconomic inequalities in lipid and glucose metabolism in early childhood in a population-based cohort: the ABCD-Study. BMC public health 2012;12:591.

135. Nobre LN, Lamounier JA, Franceschini Sdo C. Sociodemographic, anthropometric and dietary determinants of dyslipidemia in preschoolers. Jornal de pediatria 2013;89:462-9.

136. Huxley R, Neil A, Collins R. Unravelling the fetal origins hypothesis: is there really an inverse association between birthweight and subsequent blood pressure? Lancet 2002;360:659-

65.

137. Tu YK, West R, Ellison GT, Gilthorpe MS. Why evidence for the fetal origins of adult disease might be a statistical artifact: the "reversal paradox" for the relation between birth weight and blood pressure in later life. American journal of epidemiology 2005;161:27-32.

138. Gillman MW. Epidemiological challenges in studying the fetal origins of adult chronic disease. International journal of epidemiology 2002;31:294-9.

139. Maymunah AO, Kehinde O, Abidoye G, Oluwatosin A. Hypercholesterolaemia in pregnancy as a predictor of adverse pregnancy outcome. African health sciences 2014;14:967-73.

140. Steiner MJ, Skinner AC, Perrin EM. Fasting Might Not Be Necessary Before Lipid Screening: A Nationally Representative Cross-sectional Study. Pediatrics 2011;128:463-70.

\section{Chapter 3}

1. Mathers CD, Boerma T, Ma Fat D. Global and regional causes of death. British medical bulletin 2009;92:7-32.

2. World Health Organization. Global status report on noncommunicable diseases 2014. Geneva: WHO. Available at: http://www.who.int/mediacentre/factsheets/fs355/en/. 2015.

3. Mensah GA, Brown DW. An overview of cardiovascular disease burden in the United States. Health affairs 2007;26:38-48.

4. Thom T, Haase N, Rosamond W, et al. Heart disease and stroke statistics--2006 update: a report from the American Heart Association Statistics Committee and Stroke Statistics Subcommittee. Circulation 2006;113:e85-151.

5. Mozaffarian D, Benjamin EJ, Go AS, et al. Heart Disease and Stroke Statistics-2016 Update: A Report From the American Heart Association. Circulation 2016;133:e38-e360.

6. Liburd LC, Giles HW, Mensah GA. Looking through a glass, darkly: eliminating health disparities. Preventing chronic disease 2006;3:A72.

7. America's Health Ranking. United Health Foundation. Available at http://www.americashealthrankings.org. 2014. (Accessed March, 06, 2015, 
8. Gartner LM, Morton J, Lawrence RA, et al. Breastfeeding and the use of human milk. Pediatrics 2005; 115:496-506.

9. Martin RM, Ben-Shlomo Y, Gunnell D, Elwood P, Yarnell JW, Davey Smith G. Breast feeding and cardiovascular disease risk factors, incidence, and mortality: the Caerphilly study. Journal of epidemiology and community health 2005;59:121-9.

10. Groer MW, Jevitt CM, Sahebzamani F, Beckstead JW, Keefe DL. Breastfeeding status and maternal cardiovascular variables across the postpartum. Journal of women's health 2013;22:4539.

11. Schwarz EB, Ray RM, Stuebe AM, et al. Duration of lactation and risk factors for maternal cardiovascular disease. Obstetrics and gynecology 2009;113:974-82.

12. AAP. Section on Breastfeeding. Breastfeeding and the use of human milk. Pediatrics 2012;129:e827-41.

13. Breastfeeding Report Card 2014. CDC National Immunization Surveys. Division of Nutrition, Physical Activity, and Obesity, National Center for Chronic Disease Prevention and Health Promotion. Centers for Disease Control and Prevention. Atlanta, GA. Available at:

http://www.cdc.gov/breastfeeding/data/reportcard.htm. PDF:

http://www.cdc.gov/breastfeeding/pdf/2014breastfeedingreportcard.pdf. 2014. (Accessed March, 6, 2015,

14. Pediatric Nutrition Surveillance System (PedNSS) and the Pregnancy Surveillance System (PNSS). Division of Nutrition and Physical Activity. National Center for Chronic Disease

Prevention and Health Promotion. Centers for Disease Control and Prevention. United States Department of Health and Human Services. Available at: http://www.cdc.gov/pednss/index.htm. PDF: http://www.cdc.gov/pednss/pednss_tables/pdf/national table7.pdf. 2014. (Accessed March 6, 2015, at http://www.cdc.gov/pednss/pednss_tables/pdf/national_table2.pdf.)

15. Bartick M, Reinhold A. The burden of suboptimal breastfeeding in the United States: a pediatric cost analysis. Pediatrics 2010;125:e1048-56.

16. Bartick M. Breastfeeding and the U.S. economy. Breastfeeding medicine : the official journal of the Academy of Breastfeeding Medicine 2011;6:313-8.

17. Bartick MC, Stuebe AM, Schwarz EB, Luongo C, Reinhold AG, Foster EM. Cost analysis of maternal disease associated with suboptimal breastfeeding. Obstetrics and gynecology

2013;122:111-9.

18. Jwa SC, Fujiwara T, Kondo N. Latent protective effects of breastfeeding on late childhood overweight and obesity: a nationwide prospective study. Obesity 2014;22:1527-37.

19. Weng SF, Redsell SA, Swift JA, Yang M, Glazebrook CP. Systematic review and metaanalyses of risk factors for childhood overweight identifiable during infancy. Archives of disease in childhood 2012;97:1019-26.

20. von Kries R, Koletzko B, Sauerwald T, et al. Breast feeding and obesity: cross sectional study. BMJ 1999;319:147-50.

21. McCrory C, Layte R. Breastfeeding and risk of overweight and obesity at nine-years of age.

Soc Sci Med 2012;75:323-30.

22. Plagemann A, Harder T. Breast feeding and the risk of obesity and related metabolic diseases in the child. Metabolic Syndrome and Related Disorders 2005;3:222-32.

23. Amorim Rde J, Coelho AF, de Lira PI, Lima Mde C. Is breastfeeding protective for blood pressure in schoolchildren? A cohort study in northeast Brazil. Breastfeeding medicine : the official journal of the Academy of Breastfeeding Medicine 2014;9:149-56.

24. Hosaka M, Asayama K, Staessen JA, et al. Breastfeeding leads to lower blood pressure in 7year-old Japanese children: Tohoku Study of Child Development. Hypertension research : official journal of the Japanese Society of Hypertension 2013;36:117-22.

25. Lawlor DA, Najman JM, Sterne J, Williams GM, Ebrahim S, Davey Smith G. Associations of parental, birth, and early life characteristics with systolic blood pressure at 5 years of age: 
findings from the Mater-University study of pregnancy and its outcomes. Circulation 2004;110:2417-23.

26. Martin RM, Ness AR, Gunnell D, Emmett P, Davey Smith G, Team AS. Does breast-feeding in infancy lower blood pressure in childhood? The Avon Longitudinal Study of Parents and Children (ALSPAC). Circulation 2004;109:1259-66.

27. Owen CG, Whincup PH, Gilg JA, Cook DG. Effect of breast feeding in infancy on blood pressure in later life: systematic review and meta-analysis. Bmj 2003;327:1189-95.

28. Martin RM, Gunnell D, Smith GD. Breastfeeding in infancy and blood pressure in later life: systematic review and meta-analysis. American journal of epidemiology 2005;161:15-26.

29. de Jonge LL, van Osch-Gevers L, Geelhoed JJ, et al. Breastfeeding is not associated with left cardiac structures and blood pressure during the first two years of life. The Generation R Study.

Early human development 2010;86:463-8.

30. Kwok MK, Leung GM, Schooling CM. Breastfeeding and adolescent blood pressure: evidence from Hong Kong's "Children of 1997" Birth Cohort. American journal of epidemiology 2013;178:928-36.

31. Horta BL, Victora CG, Lima RC, Goncalves H, Guimaraes BE, Barros FC. Breastfeeding duration and blood pressure among Brazilian adolescents. Acta paediatrica 2006;95:325-31.

32. Fall $\mathrm{CH}$, Borja JB, Osmond $\mathrm{C}$, et al. Infant-feeding patterns and cardiovascular risk factors in young adulthood: data from five cohorts in low- and middle-income countries. International journal of epidemiology 2011;40:47-62.

33. Izadi V, Kelishadi R, Qorbani M, et al. Duration of breast-feeding and cardiovascular risk factors among Iranian children and adolescents: the CASPIAN III study. Nutrition (Burbank, Los Angeles County, Calif) 2013;29:744-51.

34. Rudnicka AR, Owen CG, Strachan DP. The effect of breastfeeding on cardiorespiratory risk factors in adult life. Pediatrics 2007;119:e1107-15.

35. Kramer MS, Matush L, Vanilovich I, et al. Effects of prolonged and exclusive breastfeeding on child height, weight, adiposity, and blood pressure at age $6.5 \mathrm{y}$ : evidence from a large randomized trial. The American journal of clinical nutrition 2007;86:1717-21.

36. Kramer MS. "Breast is best": The evidence. Early human development 2010;86:729-32.

37. Horta BL, Loret de Mola C, Victora CG. Long-term consequences of breastfeeding on cholesterol, obesity, systolic blood pressure and type 2 diabetes: a systematic review and metaanalysis. Acta paediatrica (Oslo, Norway : 1992) Supplement 2015;104:30-7.

38. Plancoulaine S, Charles MA, Lafay L, et al. Infant-feeding patterns are related to blood cholesterol concentration in prepubertal children aged 5-11 y: the Fleurbaix-Laventie Ville Sante study. European journal of clinical nutrition 2000;54:114-9.

39. Thorsdottir I, Gunnarsdottir I, Palsson GI. Association of birth weight and breast-feeding with coronary heart disease risk factors at the age of 6 years. Nutrition, metabolism, and cardiovascular diseases : NMCD 2003;13:267-72.

40. Pearce MS, Relton CL, Parker L, Unwin NC. Sex differences in the association between infant feeding and blood cholesterol in later life: the Newcastle thousand families cohort study at age 49-51 years. European journal of epidemiology 2009;24:375-80.

41. Owen CG, Whincup PH, Kaye SJ, et al. Does initial breastfeeding lead to lower blood cholesterol in adult life? A quantitative review of the evidence. The American journal of clinical nutrition 2008;88:305-14.

42. Bekkers MB, Brunekreef B, Smit HA, et al. Early-life determinants of total and HDL cholesterol concentrations in 8-year-old children; the PIAMA birth cohort study. PloS one 2011;6:e25533.

43. Owen CG, Whincup PH, Odoki K, Gilg JA, Cook DG. Infant feeding and blood cholesterol: a study in adolescents and a systematic review. Pediatrics 2002;110:597-608.

44. Victora CG, Horta BL, Post P, et al. Breast feeding and blood lipid concentrations in male Brazilian adolescents. Journal of epidemiology and community health 2006;60:621-5. 
45. Kolacek S, Kapetanovic T, Zimolo A, Luzar V. Early determinants of cardiovascular risk factors in adults. A. Plasma lipids. Acta paediatrica 1993;82:699-704.

46. Ip S, Chung M, Raman G, et al. Breastfeeding and maternal and infant health outcomes in developed countries. Evid Rep Technol Assess (Full Rep) 2007:1-186.

47. Toma TS, Rea MF. [Benefits of breastfeeding for maternal and child health: an essay on the scientific evidence]. Cadernos de saude publica 2008;24 Suppl 2:S235-46.

48. Ram KT, Bobby P, Hailpern SM, et al. Duration of lactation is associated with lower prevalence of the metabolic syndrome in midlife--SWAN, the study of women's health across the nation. American journal of obstetrics and gynecology 2008;198:268 e1-6.

49. Natland Fagerhaug T, Forsmo S, Jacobsen GW, Midthjell K, Andersen LF, Ivar Lund Nilsen T. A prospective population-based cohort study of lactation and cardiovascular disease mortality: the HUNT study. BMC public health 2013;13:1070.

50. Rooney BL, Schauberger CW, Mathiason MA. Impact of perinatal weight change on longterm obesity and obesity-related illnesses. Obstetrics and gynecology 2005;106:1349-56.

51. Ebina S, Kashiwakura I. Influence of breastfeeding on maternal blood pressure at one month postpartum. International journal of women's health 2012;4:333-9.

52. Lupton SJ, Chiu CL, Lujic S, Hennessy A, Lind JM. Association between parity and breastfeeding with maternal high blood pressure. American journal of obstetrics and gynecology 2013;208:454 e1-7.

53. McClure CK, Schwarz EB, Conroy MB, Tepper PG, Janssen I, Sutton-Tyrrell KC. Breastfeeding and subsequent maternal visceral adiposity. Obesity 2011;19:2205-13.

54. McClure CK, Catov JM, Ness RB, Schwarz EB. Lactation and maternal subclinical cardiovascular disease among premenopausal women. American journal of obstetrics and gynecology 2012;207:46 e1-8.

55. Oken E, Patel R, Guthrie LB, et al. Effects of an intervention to promote breastfeeding on maternal adiposity and blood pressure at 11.5 y postpartum: results from the Promotion of Breastfeeding Intervention Trial, a cluster-randomized controlled trial. The American journal of clinical nutrition 2013;98:1048-56.

56. Stuebe AM, Kleinman K, Gillman MW, Rifas-Shiman SL, Gunderson EP, Rich-Edwards J. Duration of lactation and maternal metabolism at 3 years postpartum. Journal of women's health 2010;19:941-50.

57. Eidelman AI. Breastfeeding and the use of human milk: an analysis of the American Academy of Pediatrics 2012 Breastfeeding Policy Statement. Breastfeeding medicine : the official journal of the Academy of Breastfeeding Medicine 2012;7:323-4.

58. Ballard O, Morrow AL. Human milk composition: nutrients and bioactive factors. Pediatric clinics of North America 2013;60:49-74.

59. Schanler RJ. Evaluation of the evidence to support current recommendations to meet the needs of premature infants: the role of human milk. The American journal of clinical nutrition 2007;85:625S-8S.

60. Lawrence PB. Breast milk. Best source of nutrition for term and preterm infants. Pediatric clinics of North America 1994;41:925-41.

61. Hamosh M. Should infant formulas be supplemented with bioactive components and conditionally essential nutrients present in human milk? The Journal of nutrition 1997;127:971S$4 \mathrm{~S}$.

62. Stevens EE, Patrick TE, Pickler R. A history of infant feeding. The Journal of perinatal education 2009;18:32-9.

63. Lucas A, Lucas PJ, Baum JD. Pattern of milk flow in breast-fed infants. Lancet (London, England) 1979;2:57-8.

64. Hamosh M. Does infant nutrition affect adiposity and cholesterol levels in the adult? Journal of pediatric gastroenterology and nutrition 1988;7:10-6. 
65. Hediger ML, Overpeck MD, Kuczmarski RJ, Ruan WJ. Association between infant breastfeeding and overweight in young children. Jama 2001;285:2453-60.

66. Ravelli AC, van der Meulen JH, Osmond C, Barker DJ, Bleker OP. Infant feeding and adult glucose tolerance, lipid profile, blood pressure, and obesity. Archives of disease in childhood 2000;82:248-52.

67. Arenz S, Ruckerl R, Koletzko B, von Kries R. Breast-feeding and childhood obesity--a systematic review. International journal of obesity and related metabolic disorders : journal of the International Association for the Study of Obesity 2004;28:1247-56.

68. Baker RS, Foote J, Kemmeter P, Brady R, Vroegop T, Serveld M. The science of stapling and leaks. Obesity surgery 2004;14:1290-8.

69. Li R, Scanlon KS, May A, Rose C, Birch L. Bottle-feeding practices during early infancy and eating behaviors at 6 years of age. Pediatrics 2014;134 Suppl 1:S70-7.

70. Stuebe AM, Rich-Edwards JW. The reset hypothesis: lactation and maternal metabolism. American journal of perinatology 2009;26:81-8.

71. Garza R, Ramussen, K.M. . Pregnancy and lactation. In J. S. Garrow, W. P. T. James, \& A. Ralph (Eds.), Human nutrition and dietetics (10th ed., pp. 437-448). Edinburgh, Scotland: Churchill-Livingstone.2000.

72. Butte NF, Wong WW, Hopkinson JM. Energy requirements of lactating women derived from doubly labeled water and milk energy output. The Journal of nutrition 2001;131:53-8.

73. Gunderson EP, Lewis CE, Wei GS, Whitmer RA, Quesenberry CP, Sidney S. Lactation and changes in maternal metabolic risk factors. Obstetrics and gynecology 2007;109:729-38.

74. Kallio MJ, Siimes MA, Perheentupa J, Salmenpera L, Miettinen TA. Serum cholesterol and lipoprotein concentrations in mothers during and after prolonged exclusive lactation. Metabolism: clinical and experimental 1992;41:1327-30.

75. Knopp RH, Walden CE, Wahl PW, et al. Effect of postpartum lactation on lipoprotein lipids and apoproteins. The Journal of clinical endocrinology and metabolism 1985;60:542-7.

76. Crispim PA, Peixoto Mdo R, Jardim PC. Risk factors associated with high blood pressure in two-to five-year-old children. Arquivos brasileiros de cardiologia 2014;102:39-46.

77. McMurray RG, Harrell JS, Levine AA, Gansky SA. Childhood obesity elevates blood pressure and total cholesterol independent of physical activity. International journal of obesity and related metabolic disorders : journal of the International Association for the Study of Obesity 1995; 19:881-6.

78. Morrison JA, Sprecher DL, Barton BA, Waclawiw MA, Daniels SR. Overweight, fat patterning, and cardiovascular disease risk factors in black and white girls: The National Heart, Lung, and Blood Institute Growth and Health Study. The Journal of pediatrics 1999;135:458-64. 79. West Virginia Birth Score Project. This program is funded under an agreement with the West Virginia Department of Health and Human Resources, Bureau for Public Health, Office of Maternal, Child and Family Health. Available at http://www.wvdhhr.org/birthscore/. 2013. at http://www.wvdhhr.org/birthscore/.)

80. Mullett MD, Cottrell L, Lilly C, et al. Association between birth characteristics and coronary disease risk factors among fifth graders. The Journal of pediatrics 2014;164:78-82.

81. Myerberg DZ, Carpenter RG, Myerberg CF, Britton CM, Bailey CW, Fink BE. Reducing postneonatal mortality in West Virginia: a statewide intervention program targeting risk identified at and after birth. Am J Public Health 1995;85:631-7.

82. Muratova VN, Demerath EW, Spangler E, et al. The relation of obesity to cardiovascular risk factors among children: the CARDIAC project. W V Med J 2002;98:263-7.

83. Cottrell L, John C, Murphy E, et al. Individual-, family-, community-, and policy-level impact of a school-based cardiovascular risk detection screening program for children in underserved, rural areas: the CARDIAC Project. J Obes 2013;2013:732579.

84. Harris CV, Neal WA. Assessing BMI in West Virginia schools: parent perspectives and the influence of context. Pediatrics 2009;124 Suppl 1:S63-72. 
85. Demerath E, Muratova V, Spangler E, Li J, Minor VE, Neal WA. School-based obesity screening in rural Appalachia. Prev Med 2003;37:553-60.

86. Ice CL, Murphy E, Minor VE, Neal WA. Metabolic syndrome in fifth grade children with acanthosis nigricans: results from the CARDIAC project. World journal of pediatrics : WJP 2009;5:23-30.

87. Must A, Anderson SE. Body mass index in children and adolescents: considerations for population-based applications. International journal of obesity 2006;30:590-4.

88. Kuczmarski RJ, Ogden CL, Guo SS, et al. 2000 CDC Growth Charts for the United States: methods and development. Vital and health statistics Series 11, Data from the national health survey 2002:1-190.

89. Hammer LD, Kraemer HC, Wilson DM, Ritter PL, Dornbusch SM. Standardized percentile curves of body-mass index for children and adolescents. American journal of diseases of children 1991;145:259-63.

90. U.S. Census Bureau: State and County QuickFacts. Data derived from Population Estimates, American Community Survey, Census of Population and Housing, State and County Housing Unit Estimates, County Business Patterns, Nonemployer Statistics, Economic Census, Survey of Business Owners, Building Permits vol. 2014. 2011.

91. Roberts RJ, Leary SD, Smith GD, Ness AR, Team AS. Maternal age in pregnancy and offspring blood pressure in childhood in the Avon Longitudinal Study of Parents and Children (ALSPAC). Journal of human hypertension 2005;19:893-900.

92. Savage T, Derraik JG, Miles HL, Mouat F, Hofman PL, Cutfield WS. Increasing maternal age is associated with taller stature and reduced abdominal fat in their children. PloS one 2013;8:e58869.

93. van den Berg G, van Eijsden M, Galindo-Garre F, Vrijkotte TG, Gemke RJ. Explaining socioeconomic inequalities in childhood blood pressure and prehypertension: the ABCD study. Hypertension 2013;61:35-41.

94. Bertini G, Perugi S, Dani C, Pezzati M, Tronchin M, Rubaltelli FF. Maternal education and the incidence and duration of breast feeding: a prospective study. Journal of pediatric gastroenterology and nutrition 2003;37:447-52.

95. van Rossem L, Oenema A, Steegers EA, et al. Are starting and continuing breastfeeding related to educational background? The generation R study. Pediatrics 2009;123:e1017-27. 96. Burke V, Beilin LJ, Simmer K, et al. Breastfeeding and overweight: longitudinal analysis in an Australian birth cohort. The Journal of pediatrics 2005;147:56-61.

97. Colen CG, Ramey DM. Is breast truly best? Estimating the effects of breastfeeding on longterm child health and wellbeing in the United States using sibling comparisons. Soc Sci Med 2014;109:55-65.

98. Dekkers JC, Treiber FA, Kapuku G, Snieder H. Differential influence of family history of hypertension and premature myocardial infarction on systolic blood pressure and left ventricular mass trajectories in youth. Pediatrics 2003;111:1387-93.

99. Bergstrom E, Hernell O, Persson LA, Vessby B. Serum lipid values in adolescents are related to family history, infant feeding, and physical growth. Atherosclerosis 1995;117:1-13.

100. Gaillard R, Rurangirwa AA, Williams MA, et al. Maternal parity, fetal and childhood growth, and cardiometabolic risk factors. Hypertension 2014;64:266-74.

101. Whincup PH, Cook DG, Papacosta O. Do maternal and intrauterine factors influence blood pressure in childhood? Archives of disease in childhood 1992;67:1423-9.

102. Huang RC, Mori TA, Beilin LJ. Early life programming of cardiometabolic disease in the Western Australian pregnancy cohort (Raine) study. Clinical and experimental pharmacology \& physiology 2012;39:973-8.

103. Giglia R, Binns CW, Alfonso H. Maternal cigarette smoking and breastfeeding duration. Acta paediatrica (Oslo, Norway : 1992) 2006;95:1370-4. 
104. Scheers Andersson E, Tynelius P, Nohr EA, Sorensen TI, Rasmussen F. No association of maternal gestational weight gain with offspring blood pressure and hypertension at age 18 years in male sibling-pairs: a prospective register-based cohort study. PloS one 2015;10:e0121202.

105. Mamun AA, O'Callaghan M, Callaway L, Williams G, Najman J, Lawlor DA. Associations of gestational weight gain with offspring body mass index and blood pressure at 21 years of age: evidence from a birth cohort study. Circulation 2009;119:1720-7.

106. Behrman RE, Butler, A. S. . Neurodevelopmental, Health, and Family Outcomes for Infants Born Preterm. Preterm Birth: Causes, Consequences, and Prevention. Washington (DC). 2007, National Academy of Sciences.; 2007.

107. Alexander GR. Prematurity at Birth: Determinants, Consequences, and Geographic Variation Preterm Birth: Causes, Consequences, and Prevention. Washington (DC). National Academy of Sciences.; 2007.

108. Hjalmarson O, Sandberg K. Abnormal lung function in healthy preterm infants. American journal of respiratory and critical care medicine 2002;165:83-7.

109. Moreira RS, Magalhaes LC, Alves CR. Effect of preterm birth on motor development, behavior, and school performance of school-age children: a systematic review. Jornal de pediatria 2014;90:119-34.

110. Wilcox AJ. On the importance--and the unimportance--of birthweight. International journal of epidemiology 2001;30:1233-41.

111. Wright RW, Brand RA, Dunn W, Spindler KP. How to write a systematic review. Clin Orthop Relat Res 2007;455:23-9.

112. Higgins JPT, Green, S. Cochrane Handbook for Systematic Reviews of Interventions Version 5.1.0.: The Cochrane Collaboration; 2011.; 2011.

113. Garritty C, Tsertsvadze A, Tricco AC, Sampson M, Moher D. Updating systematic reviews: an international survey. PLoS One 2010;5:e9914.

114. Ramirez-Silva I, Rivera JA, Trejo-Valdivia B, et al. Breastfeeding status at age 3 months is associated with adiposity and cardiometabolic markers at age 4 years in Mexican children. The Journal of nutrition 2015;145:1295-302.

115. Umer A, Hamilton C, Britton CM, et al. Association between Breastfeeding and Childhood Obesity: Analysis of a Linked Longitudinal Study of Rural Appalachian Fifth-Grade Children. Childhood obesity 2015;11:449-55.

116. Kark JD, Troya G, Friedlander Y, Slater PE, Stein Y. Validity of maternal reporting of breast feeding history and the association with blood lipids in 17 year olds in Jerusalem. Journal of epidemiology and community health 1984;38:218-25.

117. Gishti O, Gaillard R, Durmus B, et al. Infant diet and metabolic outcomes in school-age children. The Generation R Study. European journal of clinical nutrition 2014;68:1008-15. 118. Frerichs RR, Srinivasan SR, Webber LS, Berenson GR. Serum cholesterol and triglyceride levels in 3,446 children from a biracial community: the Bogalusa Heart Study. Circulation 1976;54:302-9.

119. Miller M, Stone NJ, Ballantyne C, et al. Triglycerides and cardiovascular disease: a scientific statement from the American Heart Association. Circulation 2011;123:2292-333. 120. Centers for Disease C, Prevention. Prevalence of abnormal lipid levels among youths --United States, 1999-2006. MMWR Morbidity and mortality weekly report 2010;59:29-33. 121. Dai S, Fulton JE, Harrist RB, Grunbaum JA, Steffen LM, Labarthe DR. Blood lipids in children: age-related patterns and association with body-fat indices: Project HeartBeat! American journal of preventive medicine 2009;37:S56-64.

122. Sumner AE. Ethnic differences in triglyceride levels and high-density lipoprotein lead to underdiagnosis of the metabolic syndrome in black children and adults. The Journal of pediatrics 2009;155:S7 e-11. 
123. van den Berg G, van Eijsden M, Vrijkotte TG, Gemke RJ. Socioeconomic inequalities in lipid and glucose metabolism in early childhood in a population-based cohort: the ABCD-Study. BMC public health 2012;12:591.

124. Nobre LN, Lamounier JA, Franceschini Sdo C. Sociodemographic, anthropometric and dietary determinants of dyslipidemia in preschoolers. Jornal de pediatria 2013;89:462-9.

125. Haney EM, Huffman LH, Bougatsos C, et al. Screening for Lipid Disorders in Children and Adolescents. Rockville (MD)2007.

126. Pesa JA, Shelton MM. Health-enhancing behaviors correlated with breastfeeding among a national sample of mothers. Public health nursing 1999;16:120-4.

127. Chertok IR, Luo J, Culp S, Mullett M. Intent to breastfeed: a population-based perspective. Breastfeeding medicine : the official journal of the Academy of Breastfeeding Medicine 2011;6:125-9.

128. Holmes VA, Cardwell C, McKinley MC, et al. Association between breast-feeding and anthropometry and CVD risk factor status in adolescence and young adulthood: the Young Hearts Project, Northern Ireland. Public health nutrition 2010;13:771-8.

129. Steiner MJ, Skinner AC, Perrin EM. Fasting Might Not Be Necessary Before Lipid Screening: A Nationally Representative Cross-sectional Study. Pediatrics 2011;128:463-70.

\section{Chapter 4}

1. Freedman DS, Mei Z, Srinivasan SR, Berenson GS, Dietz WH. Cardiovascular risk factors and excess adiposity among overweight children and adolescents: the Bogalusa Heart Study. J Pediatr 2007;150:12-7 e2.

2. Teixeira PJ, Sardinha LB, Going SB, Lohman TG. Total and regional fat and serum cardiovascular disease risk factors in lean and obese children and adolescents. Obesity research 2001;9:432-42.

3. Celermajer DS, Ayer JG. Childhood risk factors for adult cardiovascular disease and primary prevention in childhood. Heart 2006;92:1701-6.

4. Freedman DS, Khan LK, Dietz WH, Srinivasan SR, Berenson GS. Relationship of childhood obesity to coronary heart disease risk factors in adulthood: the Bogalusa Heart Study. Pediatrics 2001;108:712-8.

5. Mozaffarian D, Benjamin EJ, Go AS, et al. Heart Disease and Stroke Statistics-2016 Update: A Report From the American Heart Association. Circulation 2016;133:e38-e360.

6. Mozaffarian D, Benjamin EJ, Go AS, et al. Heart disease and stroke statistics--2015 update: a report from the American Heart Association. Circulation 2015;131:e29-322.

7. WHO. WHO. World health statistics 2008. Geneva: World Health Organization. 2009.

8. Morabia A, Abel T. The WHO report "Preventing chronic diseases: a vital investment" and us. Soz Praventivmed 2006;51:74.

9. Lloyd-Jones D, Adams RJ, Brown TM, et al. Executive summary: heart disease and stroke statistics--2010 update: a report from the American Heart Association. Circulation 2010;121:94854.

10. Heron M. Deaths: leading causes for 2010. Natl Vital Stat Rep 2013;62:1-96.

11. Heidenreich PA, Trogdon JG, Khavjou OA, et al. Forecasting the future of cardiovascular disease in the United States: a policy statement from the American Heart Association. Circulation 2011;123:933-44.

12. Crowley DI, Khoury PR, Urbina EM, Ippisch HM, Kimball TR. Cardiovascular impact of the pediatric obesity epidemic: higher left ventricular mass is related to higher body mass index. The Journal of pediatrics 2011;158:709-14 e1. 
13. Ford ES, Mokdad AH, Ajani UA. Trends in risk factors for cardiovascular disease among children and adolescents in the United States. Pediatrics 2004;114:1534-44.

14. Clinical Guidelines on the Identification, Evaluation, and Treatment of Overweight and Obesity in Adults--The Evidence Report. National Institutes of Health. Obesity research 1998;6 Suppl 2:51S-209S.

15. Freedman DS, Dietz WH, Tang R, et al. The relation of obesity throughout life to carotid intima-media thickness in adulthood: the Bogalusa Heart Study. Int J Obes Relat Metab Disord 2004;28:159-66.

16. Baker JL, Olsen LW, Sorensen TI. Childhood body-mass index and the risk of coronary heart disease in adulthood. The New England journal of medicine 2007;357:2329-37.

17. Poirier P, Giles TD, Bray GA, et al. Obesity and cardiovascular disease: pathophysiology, evaluation, and effect of weight loss: an update of the 1997 American Heart Association

Scientific Statement on Obesity and Heart Disease from the Obesity Committee of the Council on Nutrition, Physical Activity, and Metabolism. Circulation 2006;113:898-918.

18. Lloyd LJ, Langley-Evans SC, McMullen S. Childhood obesity and risk of the adult metabolic syndrome: a systematic review. International journal of obesity 2012;36:1-11.

19. Bridger T. Childhood obesity and cardiovascular disease. Paediatr Child Health 2009;14:177-

82.

20. Friedemann C, Heneghan C, Mahtani K, Thompson M, Perera R, Ward AM. Cardiovascular disease risk in healthy children and its association with body mass index: systematic review and meta-analysis. Bmj 2012;345:e4759.

21. Fact sheets from the Surgeon General's Call to Action to Prevent and Decrease Overweight and Obesity. W V Med J 2002;98:234-43.

22. Deshmukh-Taskar P, Nicklas TA, Morales M, Yang SJ, Zakeri I, Berenson GS. Tracking of overweight status from childhood to young adulthood: the Bogalusa Heart Study. European journal of clinical nutrition 2006;60:48-57.

23. Serdula MK, Ivery D, Coates RJ, Freedman DS, Williamson DF, Byers T. Do obese children become obese adults? A review of the literature. Preventive medicine 1993;22:167-77.

24. Freedman DS, Khan LK, Serdula MK, Dietz WH, Srinivasan SR, Berenson GS. Interrelationships among childhood BMI, childhood height, and adult obesity: the Bogalusa Heart Study. Int J Obes Relat Metab Disord 2004;28:10-6.

25. Nadeau KJ, Maahs DM, Daniels SR, Eckel RH. Childhood obesity and cardiovascular disease: links and prevention strategies. Nat Rev Cardiol 2011;8:513-25.

26. Reinehr T, de Sousa G, Toschke AM, Andler W. Long-term follow-up of cardiovascular disease risk factors in children after an obesity intervention. Am J Clin Nutr 2006;84:490-6.

27. Glowinska B, Urban M, Koput A, Galar M. New atherosclerosis risk factors in obese, hypertensive and diabetic children and adolescents. Atherosclerosis 2003;167:275-86.

28. Li S, Liu X, Okada T, Iwata F, Hara M, Harada K. Serum lipid profile in obese children in China. Pediatr Int 2004;46:425-8.

29. Friedland O, Nemet D, Gorodnitsky N, Wolach B, Eliakim A. Obesity and lipid profiles in children and adolescents. J Pediatr Endocrinol Metab 2002;15:1011-6.

30. Mayor S. Childhood obesity increases blood pressure in adolescence, study shows. Bmj 2012;344:e3065.

31. Muntner P, He J, Cutler JA, Wildman RP, Whelton PK. Trends in blood pressure among children and adolescents. JAMA 2004;291:2107-13.

32. Guardamagna O, Baracco V, Abello F, Bona G. Identification and management of dyslipidemic children. Minerva Pediatr 2009;61:391-8.

33. Chen X, Wang Y. Tracking of blood pressure from childhood to adulthood: a systematic review and meta-regression analysis. Circulation 2008;117:3171-80.

34. Franklin BA, Cushman M. Recent advances in preventive cardiology and lifestyle medicine: a themed series. Circulation 2011;123:2274-83. 
35. Jolliffe CJ, Janssen I. Vascular risks and management of obesity in children and adolescents. Vasc Health Risk Manag 2006;2:171-87.

36. Steinberger J, Daniels SR. Obesity, insulin resistance, diabetes, and cardiovascular risk in children: an American Heart Association scientific statement from the Atherosclerosis, Hypertension, and Obesity in the Young Committee (Council on Cardiovascular Disease in the Young) and the Diabetes Committee (Council on Nutrition, Physical Activity, and Metabolism). Circulation 2003;107:1448-53.

37. Lloyd LJ, Langley-Evans SC, McMullen S. Childhood obesity and adult cardiovascular disease risk: a systematic review. International journal of obesity 2010;34:18-28.

38. Park MH, Falconer C, Viner RM, Kinra S. The impact of childhood obesity on morbidity and mortality in adulthood: a systematic review. Obes Rev 2012;13:985-1000.

39. Reilly JJ, Kelly J. Long-term impact of overweight and obesity in childhood and adolescence on morbidity and premature mortality in adulthood: systematic review. International journal of obesity 2011;35:891-8.

40. Juonala M, Magnussen CG, Berenson GS, et al. Childhood adiposity, adult adiposity, and cardiovascular risk factors. The New England journal of medicine 2011;365:1876-85.

41. Liberati A, Altman DG, Tetzlaff J, et al. The PRISMA statement for reporting systematic reviews and meta-analyses of studies that evaluate health care interventions: explanation and elaboration. Annals of internal medicine 2009;151:W65-94.

42. Virani SS. Non-HDL cholesterol as a metric of good quality of care: opportunities and challenges. Tex Heart Inst J 2011;38:160-2.

43. Emerging Risk Factors C, Di Angelantonio E, Sarwar N, et al. Major lipids, apolipoproteins, and risk of vascular disease. JAMA 2009;302:1993-2000.

44. Reilly JJ, Methven E, McDowell ZC, et al. Health consequences of obesity. Arch Dis Child 2003;88:748-52.

45. Higgins JPT, Green, S. Cochrane Handbook for Systematic Reviews of Interventions Version 5.1.0.: The Cochrane Collaboration; 2011.; 2011.

46. Garritty C, Tsertsvadze A, Tricco AC, Sampson M, Moher D. Updating systematic reviews: an international survey. PloS one 2010;5:e9914.

47. Javed A, Jumean M, Murad MH, et al. Diagnostic performance of body mass index to identify obesity as defined by body adiposity in children and adolescents: a systematic review and metaanalysis. Pediatr Obes 2014.

48. Brambilla P, Bedogni G, Heo M, Pietrobelli A. Waist circumference-to-height ratio predicts adiposity better than body mass index in children and adolescents. International journal of obesity 2013;37:943-6.

49. Shea BJ, Hamel C, Wells GA, et al. AMSTAR is a reliable and valid measurement tool to assess the methodological quality of systematic reviews. J Clin Epidemiol 2009;62:1013-20. 50. Wright RW, Brand RA, Dunn W, Spindler KP. How to write a systematic review. Clin Orthop Relat Res 2007;455:23-9.

51. Cook DJ, Mulrow CD, Haynes RB. Systematic reviews: synthesis of best evidence for clinical decisions. Annals of internal medicine 1997;126:376-80.

52. Reeves BC, Deeks, J. J., Higgins, J. P.T., Wells, G.A. . Chapter 13: Including nonrandomized studies. In: Higgins JPT, Green S (editors), Cochrane Handbook for Systematic Reviews of Interventions. Version 5.0.1 The Cochrane Collaboration, 2008. Available from http://www.cochrane-handbook.org.2008.

53. Moher D, Liberati A, Tetzlaff J, Altman DG. Preferred reporting items for systematic reviews and meta-analyses: the PRISMA statement. Bmj 2009;339:b2535.

54. Panic N, Leoncini E, de Belvis G, Ricciardi W, Boccia S. Evaluation of the endorsement of the preferred reporting items for systematic reviews and meta-analysis (PRISMA) statement on the quality of published systematic review and meta-analyses. PloS one 2013;8:e83138. 
55. (Available from

http://www.crd.york.ac.uk/PROSPERO/display_record.asp?ID=CRD42015019763). . 2015.

56. Greenhalgh T. Papers that summarise other papers (systematic reviews and meta-analyses). Bmj 1997;315:672-5.

57. Must A, Anderson SE. Body mass index in children and adolescents: considerations for population-based applications. International journal of obesity 2006;30:590-4.

58. Rothstein HR, Sutton, A. J., Borenstein, M. Publication Bias in Meta-Analysis - Prevention, Assessment and Adjustments. 2005 John Wiley \& Sons, Ltd2005.

59. Littell JH, Corcoran. J., Pillai, V. Systematic reviews and meta-analysis, Oxford University Press, New York (2008), p. 202 ISBN: 978-0-19-532654-32008.

60. Conn VS, Valentine JC, Cooper HM, Rantz MJ. Grey literature in meta-analyses. Nurs Res 2003;52:256-61.

61. McAuley L, Pham B, Tugwell P, Moher D. Does the inclusion of grey literature influence estimates of intervention effectiveness reported in meta-analyses? Lancet 2000;356:1228-31.

62. Hopewell S, McDonald S, Clarke M, Egger M. Grey literature in meta-analyses of randomized trials of health care interventions. Cochrane Database Syst Rev 2007:MR000010. 63. Martin JL, Perez V, Sacristan M, Alvarez E. Is grey literature essential for a better control of publication bias in psychiatry? An example from three meta-analyses of schizophrenia. Eur Psychiatry 2005;20:550-3.

64. Sacks HS, Reitman D, Pagano D, Kupelnick B. Meta-analysis: an update. Mt Sinai J Med 1996;63:216-24.

65. Yuan Y, Hunt RH. Systematic reviews: the good, the bad, and the ugly. Am J Gastroenterol 2009;104:1086-92.

66. Moher D, Cook DJ, Eastwood S, Olkin I, Rennie D, Stroup DF. Improving the quality of reports of meta-analyses of randomised controlled trials: the QUOROM statement. Quality of Reporting of Meta-analyses. Lancet 1999;354:1896-900.

67. Garg AX, Hackam D, Tonelli M. Systematic review and meta-analysis: when one study is just not enough. Clin J Am Soc Nephrol 2008;3:253-60.

68. Sampson M, McGowan J, Cogo E, Grimshaw J, Moher D, Lefebvre C. An evidence-based practice guideline for the peer review of electronic search strategies. J Clin Epidemiol 2009;62:944-52.

69. Yusuf S, Hawken S, Ounpuu S, et al. Effect of potentially modifiable risk factors associated with myocardial infarction in 52 countries (the INTERHEART study): case-control study. Lancet 2004;364:937-52.

70. Cohen J. Weighted kappa: nominal scale agreement with provision for scaled disagreement or partial credit. Psychol Bull 1968;70:213-20.

71. Microsoft Excel. Redmond, WA: Microsoft Corporation. . 2011.

72. Viswanathan M, Ansari MT, Berkman ND, et al. Assessing the Risk of Bias of Individual Studies in Systematic Reviews of Health Care Interventions. 2008.

73. Downs SH, Black N. The feasibility of creating a checklist for the assessment of the methodological quality both of randomised and non-randomised studies of health care interventions. Journal of epidemiology and community health 1998;52:377-84.

74. Reisch JS, Tyson JE, Mize SG. Aid to the evaluation of therapeutic studies. Pediatrics $1989 ; 84: 815-27$.

75. Zaza S, Carande-Kulis VG, Sleet DA, et al. Methods for conducting systematic reviews of the evidence of effectiveness and economic efficiency of interventions to reduce injuries to motor vehicle occupants. Am J Prev Med 2001;21:23-30.

76. Deeks JJ, Dinnes J, D'Amico R, et al. Evaluating non-randomised intervention studies. Health Technol Assess 2003;7:iii-x, 1-173.

77. da Costa BR, Cevallos M, Altman DG, Rutjes AW, Egger M. Uses and misuses of the STROBE statement: bibliographic study. BMJ Open 2011;1:e00048. 
78. von Elm E, Altman DG, Egger M, et al. The Strengthening the Reporting of Observational Studies in Epidemiology (STROBE) Statement: Guidelines for reporting observational studies. Int J Surg 2014;12:1495-9.

79. Borenstein M, Hedges, L. V., Higgins, J. P. T., Rothstein, H. R. . Introduction to MetaAnalysis. C 2009 John Wiley \& Sons, Ltd. ISBN: 978-0-470-05724-72009.

80. Biostat. Comprehensive Meta-Analysis (3.3) Englewood, NJ, USA: Biostat; 2015

81. Peterson RA, Brown SP. On the use of beta coefficients in meta-analysis. J Appl Psychol 2005;90:175-81.

82. DerSimonian R, Laird N. Meta-analysis in clinical trials. Control Clin Trials 1986;7:177-88. 83. Ades AE, Lu G, Higgins JP. The interpretation of random-effects meta-analysis in decision models. Med Decis Making 2005;25:646-54.

84. Borenstein M, Hedges, L. V., Higgins, J. P. T., \& Rothstein, H. R. A basic introduction to fixed effect and random effects models for meta-analysis. Research Synthesis Methods, 1, 97111.2010.

85. Egger M, Smith, G., Altman, D Systematic reviews in health care: meta-analysis in context: BMJ Books London, England.; 2001.

86. Higgins JP, Thompson SG. Quantifying heterogeneity in a meta-analysis. Stat Med 2002;21:1539-58.

87. Higgins JP, Thompson SG, Deeks JJ, Altman DG. Measuring inconsistency in meta-analyses. Bmj 2003;327:557-60.

88. Hopewell S, Loudon K, Clarke MJ, Oxman AD, Dickersin K. Publication bias in clinical trials due to statistical significance or direction of trial results. Cochrane Database Syst Rev 2009:MR000006.

89. Sterne JA, Sutton AJ, Ioannidis JP, et al. Recommendations for examining and interpreting funnel plot asymmetry in meta-analyses of randomised controlled trials. Bmj 2011;343:d4002. 90. Egger M, Davey Smith G, Schneider M, Minder C. Bias in meta-analysis detected by a simple, graphical test. Bmj 1997;315:629-34.

91. Higgins JP, Thompson SG, Spiegelhalter DJ. A re-evaluation of random-effects metaanalysis. Journal of the Royal Statistical Society Series A 2009;172:137-59.

92. Song F, Khan KS, Dinnes J, Sutton AJ. Asymmetric funnel plots and publication bias in meta-analyses of diagnostic accuracy. International journal of epidemiology 2002;31:88-95. 93. Higgins JP. Commentary: Heterogeneity in meta-analysis should be expected and appropriately quantified. International journal of epidemiology 2008;37:1158-60.

94. Lau J, Schmid CH, Chalmers TC. Cumulative meta-analysis of clinical trials builds evidence for exemplary medical care. Journal of clinical epidemiology 1995;48:45-57; discussion 9-60. 95. Abraham S, Collins G, Nordsieck M. Relationship of childhood weight status to morbidity in adults. HSMHA health reports 1971;86:273-84.

96. Barker DJ, Osmond C, Forsen TJ, Kajantie E, Eriksson JG. Trajectories of growth among children who have coronary events as adults. The New England journal of medicine 2005;353:1802-9.

97. Berkey CS, Gardner J, Colditz GA. Blood pressure in adolescence and early adulthood related to obesity and birth size. Obesity research 1998;6:187-95.

98. Eisenmann JC, Wickel EE, Welk GJ, Blair SN. Relationship between adolescent fitness and fatness and cardiovascular disease risk factors in adulthood: the Aerobics Center Longitudinal Study (ACLS). American heart journal 2005;149:46-53.

99. Graversen L, Sorensen TI, Petersen L, et al. Preschool weight and body mass index in relation to central obesity and metabolic syndrome in adulthood. PloS one 2014;9:e89986.

100. Gustafsson PE, Persson M, Hammarstrom A. Life course origins of the metabolic syndrome in middle-aged women and men: the role of socioeconomic status and metabolic risk factors in adolescence and early adulthood. Annals of epidemiology 2011;21:103-10. 
101. Holland FJ, Stark O, Ades AE, Peckham CS. Birth weight and body mass index in childhood, adolescence, and adulthood as predictors of blood pressure at age 36. Journal of epidemiology and community health 1993;47:432-5.

102. Kanade A, Deshpande S, Patil K, Rao S. Prevalence of high blood pressure among young rural adults in relation to height in childhood and adult body mass index. Journal of the American College of Nutrition 2011;30:216-23.

103. Klumbiene J, Sileikiene L, Milasauskiene Z, Zaborskis A, Shatchkute A. The relationship of childhood to adult blood pressure: longitudinal study of juvenile hypertension in Lithuania.

Journal of hypertension 2000;18:531-8.

104. Kneisley J, Schork N, Julius S. Predictors of blood pressure and hypertension in Tecumseh, Michigan. Clinical and experimental hypertension Part A, Theory and practice 1990;12:693-708. 105. Koziel S, Lipowicz A, Hulanicka B. Childhood and adolescent body fat and its relationship with health outcome in 50 year old males and females: the Wroclaw Growth Study. Collegium antropologicum 2011;35:275-80.

106. Lauer RM, Clarke WR, Mahoney LT, Witt J. Childhood predictors for high adult blood pressure. The Muscatine Study. Pediatric clinics of North America 1993;40:23-40.

107. Li L, Law C, Power C. Body mass index throughout the life-course and blood pressure in mid-adult life: a birth cohort study. Journal of hypertension 2007;25:1215-23.

108. Liddle K, O'Callaghan M, Mamun A, Najman J, Williams G. Comparison of body mass index and triceps skinfold at 5 years and young adult body mass index, waist circumference and blood pressure. Journal of paediatrics and child health 2012;48:424-9.

109. Lyngdoh T, Viswanathan B, van Wijngaarden E, Myers GJ, Bovet P. Cross-Sectional and Longitudinal Associations between Body Mass Index and Cardiometabolic Risk Factors in Adolescents in a Country of the African Region. International journal of endocrinology 2013;2013:801832.

110. Miura K, Nakagawa H, Tabata M, Morikawa Y, Nishijo M, Kagamimori S. Birth weight, childhood growth, and cardiovascular disease risk factors in Japanese aged 20 years. American journal of epidemiology 2001;153:783-9.

111. Pinto Pereira SM, Power C. Life course body mass index, birthweight and lipid levels in mid-adulthood: a nationwide birth cohort study. European heart journal 2013;34:1215-24.

112. Porkka KV, Viikari JS, Taimela S, Dahl M, Akerblom HK. Tracking and predictiveness of serum lipid and lipoprotein measurements in childhood: a 12-year follow-up. The Cardiovascular Risk in Young Finns study. American journal of epidemiology 1994;140:1096-110.

113. Schmidt MD, Dwyer T, Magnussen CG, Venn AJ. Predictive associations between alternative measures of childhood adiposity and adult cardio-metabolic health. International journal of obesity 2011;35:38-45.

114. Skidmore PM, Hardy RJ, Kuh DJ, Langenberg C, Wadsworth ME. Life course body size and lipid levels at 53 years in a British birth cohort. Journal of epidemiology and community health 2007;61:215-20.

115. Weitz CA, Friedlaender FY, Friedlaender JS. Adult lipids associated with early life growth in traditional Melanesian societies undergoing rapid modernization: a longitudinal study of the mid-20th century. American journal of physical anthropology 2014;153:551-8.

116. Wright CM, Parker L, Lamont D, Craft AW. Implications of childhood obesity for adult health: findings from thousand families cohort study. Bmj 2001;323:1280-4.

117. Guddat C, Grouven U, Bender R, Skipka G. A note on the graphical presentation of prediction intervals in random-effects meta-analyses. Systematic reviews 2012;1:34.

118. Riley RD, Higgins JP, Deeks JJ. Interpretation of random effects meta-analyses. Bmj 2011;342:d549.

119. Kristman V, Manno M, Cote P. Loss to follow-up in cohort studies: how much is too much? European journal of epidemiology 2004;19:751-60.

120. Dettori JR. Loss to follow-up. Evidence-based spine-care journal 2011;2:7-10. 
121. Tu YK, West R, Ellison GT, Gilthorpe MS. Why evidence for the fetal origins of adult disease might be a statistical artifact: the "reversal paradox" for the relation between birth weight and blood pressure in later life. American journal of epidemiology 2005;161:27-32.

122. Gillman MW. Re: "Why evidence for the fetal origins of adult disease might be a statistical artifact: the 'reversal paradox' for the relation between birth weight and blood pressure in later life". American journal of epidemiology 2005;162:292; author reply -3.

123. Cole TJ. Re: "Why evidence for the fetal origins of adult disease might be a statistical artifact: the 'reversal paradox' for the relation between birth weight and blood pressure in later life". American journal of epidemiology 2005;162:394-5; author reply 5.

124. Gillman MW. Epidemiological challenges in studying the fetal origins of adult chronic disease. International journal of epidemiology 2002;31:294-9.

125. Burt Solorzano CM, McCartney CR. Obesity and the pubertal transition in girls and boys. Reproduction 2010;140:399-410.

126. Prentice P, Viner RM. Pubertal timing and adult obesity and cardiometabolic risk in women and men: a systematic review and meta-analysis. International journal of obesity 2013;37:103643.

127. Rothman KJ. No adjustments are needed for multiple comparisons. Epidemiology 1990;1:43-6.

128. Israeli E, Korzets Z, Tekes-Manova D, et al. Blood-pressure categories in adolescence predict development of hypertension in accordance with the European guidelines. Am J Hypertens 2007;20:705-9.

129. Barker DJ, Eriksson JG, Forsen T, Osmond C. Fetal origins of adult disease: strength of effects and biological basis. International journal of epidemiology 2002;31:1235-9.

130. Barker DJ, Forsen T, Eriksson JG, Osmond C. Growth and living conditions in childhood and hypertension in adult life: a longitudinal study. Journal of hypertension 2002;20:1951-6. 131. Field AE, Cook NR, Gillman MW. Weight status in childhood as a predictor of becoming overweight or hypertensive in early adulthood. Obesity research 2005;13:163-9.

132. Salonen MK, Kajantie E, Osmond C, et al. Role of childhood growth on the risk of metabolic syndrome in obese men and women. Diabetes Metab 2009;35:94-100.

133. Sinaiko AR, Donahue RP, Jacobs DR, Jr., Prineas RJ. Relation of weight and rate of increase in weight during childhood and adolescence to body size, blood pressure, fasting insulin, and lipids in young adults. The Minneapolis Children's Blood Pressure Study. Circulation 1999;99:1471-6.

134. Lauer RM, Lee J, Clarke WR. Factors affecting the relationship between childhood and adult cholesterol levels: the Muscatine Study. Pediatrics 1988;82:309-18.

135. Hardy R, Wadsworth ME, Langenberg C, Kuh D. Birthweight, childhood growth, and blood pressure at 43 years in a British birth cohort. International journal of epidemiology 2004;33:1219.

136. Lauer RM, Clarke WR. Childhood risk factors for high adult blood pressure: the Muscatine Study. Pediatrics 1989;84:633-41.

137. Burke V, Beilin LJ, Dunbar D, Kevan M. Associations between blood pressure and overweight defined by new standards for body mass index in childhood. Prev Med 2004;38:55864.

138. Ford CA, Nonnemaker JM, Wirth KE. The influence of adolescent body mass index, physical activity, and tobacco use on blood pressure and cholesterol in young adulthood. $\mathrm{J}$ Adolesc Health 2008;43:576-83.

139. Leeners B, Rath W, Kuse S, Irawan C, Neumaier-Wagner P. The significance of under- or overweight during childhood as a risk factor for hypertensive diseases in pregnancy. Early Hum Dev 2006;82:663-8. 


\section{Chapter 5}

1. Mu M, Wang SF, Sheng J, et al. Birth weight and subsequent blood pressure: a meta-analysis. Archives of cardiovascular diseases 2012;105:99-113.

2. Frontini MG, Srinivasan SR, Xu J, Berenson GS. Low birth weight and longitudinal trends of cardiovascular risk factor variables from childhood to adolescence: the bogalusa heart study. BMC pediatrics 2004;4:22.

3. Huxley RR, Shiell AW, Law CM. The role of size at birth and postnatal catch-up growth in determining systolic blood pressure: a systematic review of the literature. Journal of hypertension 2000;18:815-31.

4. Gamborg M, Byberg L, Rasmussen F, et al. Birth weight and systolic blood pressure in adolescence and adulthood: meta-regression analysis of sex- and age-specific results from 20 Nordic studies. American journal of epidemiology 2007;166:634-45.

5. Zhang Z, Kris-Etherton PM, Hartman TJ. Birth weight and risk factors for cardiovascular disease and type 2 diabetes in US children and adolescents: 10 year results from NHANES. Maternal and child health journal 2014;18:1423-32.

6. Menezes AM, Hallal PC, Horta BL, et al. Size at birth and blood pressure in early adolescence: a prospective birth cohort study. American journal of epidemiology 2007;165:611-6.

7. Filler G, Yasin A, Kesarwani P, Garg AX, Lindsay R, Sharma AP. Big mother or small baby: which predicts hypertension? Journal of clinical hypertension 2011;13:35-41.

8. Amorim Rde J, Coelho AF, de Lira PI, Lima Mde C. Is breastfeeding protective for blood pressure in schoolchildren? A cohort study in northeast Brazil. Breastfeeding medicine : the official journal of the Academy of Breastfeeding Medicine 2014;9:149-56.

9. Azadbakht L, Kelishadi R, Saraf-Bank S, et al. The association of birth weight with cardiovascular risk factors and mental problems among Iranian school-aged children: the CASPIAN-III study. Nutrition 2014;30:150-8.

10. Donker GA, Labarthe DR, Harrist RB, et al. Low birth weight and serum lipid concentrations at age 7-11 years in a biracial sample. American journal of epidemiology 1997;145:398-407. 11. Lauren L, Jarvelin MR, Elliott P, et al. Relationship between birthweight and blood lipid concentrations in later life: evidence from the existing literature. International journal of epidemiology 2003;32:862-76.

12. Huang RC, Burke V, Newnham JP, et al. Perinatal and childhood origins of cardiovascular disease. International journal of obesity 2007;31:236-44.

13. Yajnik CS, Joglekar CV, Pandit AN, et al. Higher offspring birth weight predicts the metabolic syndrome in mothers but not fathers 8 years after delivery: the Pune Children's Study. Diabetes 2003;52:2090-6.

14. Horta BL, Loret de Mola C, Victora CG. Long-term consequences of breastfeeding on cholesterol, obesity, systolic blood pressure and type 2 diabetes: a systematic review and metaanalysis. Acta paediatrica (Oslo, Norway : 1992) Supplement 2015;104:30-7.

15. Ramirez-Silva I, Rivera JA, Trejo-Valdivia B, et al. Breastfeeding status at age 3 months is associated with adiposity and cardiometabolic markers at age 4 years in Mexican children. The Journal of nutrition 2015;145:1295-302.

16. Jwa SC, Fujiwara T, Kondo N. Latent protective effects of breastfeeding on late childhood overweight and obesity: a nationwide prospective study. Obesity 2014;22:1527-37.

17. Weng SF, Redsell SA, Swift JA, Yang M, Glazebrook CP. Systematic review and metaanalyses of risk factors for childhood overweight identifiable during infancy. Archives of disease in childhood 2012;97:1019-26.

18. von Kries R, Koletzko B, Sauerwald T, et al. Breast feeding and obesity: cross sectional study. BMJ 1999;319:147-50. 
19. McCrory C, Layte R. Breastfeeding and risk of overweight and obesity at nine-years of age. Soc Sci Med 2012;75:323-30.

20. Umer A, Hamilton C, Britton CM, et al. Association between Breastfeeding and Childhood Obesity: Analysis of a Linked Longitudinal Study of Rural Appalachian Fifth-Grade Children. Childhood obesity 2015;11:449-55.

21. Bekkers MB, Brunekreef B, Smit HA, et al. Early-life determinants of total and HDL cholesterol concentrations in 8-year-old children; the PIAMA birth cohort study. PloS one 2011;6:e25533.

22. Owen CG, Whincup PH, Odoki K, Gilg JA, Cook DG. Infant feeding and blood cholesterol: a study in adolescents and a systematic review. Pediatrics 2002;110:597-608.

23. Kark JD, Troya G, Friedlander Y, Slater PE, Stein Y. Validity of maternal reporting of breast feeding history and the association with blood lipids in 17 year olds in Jerusalem. Journal of epidemiology and community health 1984;38:218-25.

24. Victora CG, Horta BL, Post P, et al. Breast feeding and blood lipid concentrations in male Brazilian adolescents. Journal of epidemiology and community health 2006;60:621-5.

25. Gishti O, Gaillard R, Durmus B, et al. Infant diet and metabolic outcomes in school-age children. The Generation R Study. European journal of clinical nutrition 2014;68:1008-15.

26. Ram KT, Bobby P, Hailpern SM, et al. Duration of lactation is associated with lower prevalence of the metabolic syndrome in midlife--SWAN, the study of women's health across the nation. American journal of obstetrics and gynecology 2008;198:268 e1-6.

27. Schwarz EB, Ray RM, Stuebe AM, et al. Duration of lactation and risk factors for maternal cardiovascular disease. Obstetrics and gynecology 2009;113:974-82.

28. McClure CK, Schwarz EB, Conroy MB, Tepper PG, Janssen I, Sutton-Tyrrell KC. Breastfeeding and subsequent maternal visceral adiposity. Obesity 2011;19:2205-13.

29. McClure CK, Catov JM, Ness RB, Schwarz EB. Lactation and maternal subclinical cardiovascular disease among premenopausal women. American journal of obstetrics and gynecology 2012;207:46 e1-8.

30. Stuebe AM, Rich-Edwards JW. The reset hypothesis: lactation and maternal metabolism. American journal of perinatology 2009;26:81-8.

31. Abraham S, Collins G, Nordsieck M. Relationship of childhood weight status to morbidity in adults. HSMHA health reports 1971;86:273-84.

32. Barker DJ, Osmond C, Forsen TJ, Kajantie E, Eriksson JG. Trajectories of growth among children who have coronary events as adults. The New England journal of medicine 2005;353:1802-9.

33. Berkey CS, Gardner J, Colditz GA. Blood pressure in adolescence and early adulthood related to obesity and birth size. Obesity research 1998;6:187-95.

34. Eisenmann JC, Wickel EE, Welk GJ, Blair SN. Relationship between adolescent fitness and fatness and cardiovascular disease risk factors in adulthood: the Aerobics Center Longitudinal Study (ACLS). American heart journal 2005;149:46-53.

35. Freedman DS, Khan LK, Dietz WH, Srinivasan SR, Berenson GS. Relationship of childhood obesity to coronary heart disease risk factors in adulthood: the Bogalusa Heart Study. Pediatrics 2001;108:712-8.

36. Graversen L, Sorensen TI, Petersen L, et al. Preschool weight and body mass index in relation to central obesity and metabolic syndrome in adulthood. PloS one 2014;9:e89986.

37. Gustafsson PE, Persson M, Hammarstrom A. Life course origins of the metabolic syndrome in middle-aged women and men: the role of socioeconomic status and metabolic risk factors in adolescence and early adulthood. Annals of epidemiology 2011;21:103-10.

38. Holland FJ, Stark O, Ades AE, Peckham CS. Birth weight and body mass index in childhood, adolescence, and adulthood as predictors of blood pressure at age 36. Journal of epidemiology and community health 1993;47:432-5. 
39. Kanade A, Deshpande S, Patil K, Rao S. Prevalence of high blood pressure among young rural adults in relation to height in childhood and adult body mass index. Journal of the American College of Nutrition 2011;30:216-23.

40. Klumbiene J, Sileikiene L, Milasauskiene Z, Zaborskis A, Shatchkute A. The relationship of childhood to adult blood pressure: longitudinal study of juvenile hypertension in Lithuania.

Journal of hypertension 2000;18:531-8.

41. Kneisley J, Schork N, Julius S. Predictors of blood pressure and hypertension in Tecumseh, Michigan. Clinical and experimental hypertension Part A, Theory and practice 1990;12:693-708. 42. Koziel S, Lipowicz A, Hulanicka B. Childhood and adolescent body fat and its relationship with health outcome in 50 year old males and females: the Wroclaw Growth Study. Collegium antropologicum 2011;35:275-80.

43. Lauer RM, Clarke WR, Mahoney LT, Witt J. Childhood predictors for high adult blood pressure. The Muscatine Study. Pediatric clinics of North America 1993;40:23-40.

44. Li L, Law C, Power C. Body mass index throughout the life-course and blood pressure in mid-adult life: a birth cohort study. Journal of hypertension 2007;25:1215-23.

45. Liddle K, O'Callaghan M, Mamun A, Najman J, Williams G. Comparison of body mass index and triceps skinfold at 5 years and young adult body mass index, waist circumference and blood pressure. Journal of paediatrics and child health 2012;48:424-9.

46. Lyngdoh T, Viswanathan B, van Wijngaarden E, Myers GJ, Bovet P. Cross-Sectional and Longitudinal Associations between Body Mass Index and Cardiometabolic Risk Factors in Adolescents in a Country of the African Region. International journal of endocrinology 2013;2013:801832.

47. Miura K, Nakagawa H, Tabata M, Morikawa Y, Nishijo M, Kagamimori S. Birth weight, childhood growth, and cardiovascular disease risk factors in Japanese aged 20 years. American journal of epidemiology 2001;153:783-9.

48. Pinto Pereira SM, Power C. Life course body mass index, birthweight and lipid levels in midadulthood: a nationwide birth cohort study. European heart journal 2013;34:1215-24.

49. Porkka KV, Viikari JS, Taimela S, Dahl M, Akerblom HK. Tracking and predictiveness of serum lipid and lipoprotein measurements in childhood: a 12-year follow-up. The Cardiovascular Risk in Young Finns study. American journal of epidemiology 1994;140:1096-110.

50. Schmidt MD, Dwyer T, Magnussen CG, Venn AJ. Predictive associations between alternative measures of childhood adiposity and adult cardio-metabolic health. International journal of obesity 2011;35:38-45.

51. Skidmore PM, Hardy RJ, Kuh DJ, Langenberg C, Wadsworth ME. Life course body size and lipid levels at 53 years in a British birth cohort. Journal of epidemiology and community health 2007;61:215-20.

52. Weitz CA, Friedlaender FY, Friedlaender JS. Adult lipids associated with early life growth in traditional Melanesian societies undergoing rapid modernization: a longitudinal study of the mid20th century. American journal of physical anthropology 2014;153:551-8.

53. Wright CM, Parker L, Lamont D, Craft AW. Implications of childhood obesity for adult health: findings from thousand families cohort study. Bmj 2001;323:1280-4.

54. Lloyd LJ, Langley-Evans SC, McMullen S. Childhood obesity and risk of the adult metabolic syndrome: a systematic review. International journal of obesity 2012;36:1-11.

55. Lloyd LJ, Langley-Evans SC, McMullen S. Childhood obesity and adult cardiovascular disease risk: a systematic review. International journal of obesity 2010;34:18-28.

56. Park MH, Falconer C, Viner RM, Kinra S. The impact of childhood obesity on morbidity and mortality in adulthood: a systematic review. Obesity reviews : an official journal of the International Association for the Study of Obesity 2012;13:985-1000.

57. Reilly JJ, Kelly J. Long-term impact of overweight and obesity in childhood and adolescence on morbidity and premature mortality in adulthood: systematic review. Int J Obes (Lond)

2011;35:891-8. 
58. Juonala M, Magnussen CG, Berenson GS, et al. Childhood adiposity, adult adiposity, and cardiovascular risk factors. The New England journal of medicine 2011;365:1876-85.

59. Holmes VA, Cardwell C, McKinley MC, et al. Association between breast-feeding and anthropometry and CVD risk factor status in adolescence and young adulthood: the Young Hearts Project, Northern Ireland. Public health nutrition 2010;13:771-8.

60. Roth GA, Forouzanfar MH, Moran AE, et al. Demographic and epidemiologic drivers of global cardiovascular mortality. The New England journal of medicine 2015;372:1333-41. 61. Naghavi M, and Collaborators (712). GBD 2013 Mortality and Causes of Death Collaborators. Global, regional, and national age-sex specific all-cause and cause-specific mortality for 240 causes of death, 1990-2013: a systematic analysis for the Global Burden of Disease Study 2013. Lancet 2015;385:117-71.

62. Mozaffarian D, Benjamin EJ, Go AS, et al. Heart Disease and Stroke Statistics-2016 Update: A Report From the American Heart Association. Circulation 2016;133:e38-e360.

63. Ong KL, Tso AW, Lam KS, Cheung BM. Gender difference in blood pressure control and cardiovascular risk factors in Americans with diagnosed hypertension. Hypertension 2008;51:1142-8.

64. Chobanian AV, Bakris GL, Black HR, et al. Seventh report of the Joint National Committee on Prevention, Detection, Evaluation, and Treatment of High Blood Pressure. Hypertension 2003;42:1206-52.

65. O'Donnell MJ, Xavier D, Liu L, et al. Risk factors for ischaemic and intracerebral haemorrhagic stroke in 22 countries (the INTERSTROKE study): a case-control study. Lancet 2010;376:112-23.

66. Yusuf S, Hawken S, Ounpuu S, et al. Effect of potentially modifiable risk factors associated with myocardial infarction in 52 countries (the INTERHEART study): case-control study. Lancet 2004;364:937-52.

67. Cholesterol Facts. National Center for Chronic Disease Prevention and Health Promotion, Division for Heart Disease and Stroke Prevention. Available http://www.cdc.gov/cholesterol/facts.htm. 2012. (Accessed Feb 13, 2015, 2015, 68. McGill HC, Jr., McMahan CA, Malcom GT, Oalmann MC, Strong JP. Effects of serum lipoproteins and smoking on atherosclerosis in young men and women. The PDAY Research Group. Pathobiological Determinants of Atherosclerosis in Youth. Arteriosclerosis, thrombosis, and vascular biology 1997; 17:95-106.

69. Berenson GS, Srinivasan SR, Bao W, Newman WP, 3rd, Tracy RE, Wattigney WA. Association between multiple cardiovascular risk factors and atherosclerosis in children and young adults. The Bogalusa Heart Study. The New England journal of medicine 1998;338:16506.

70. Davis PH, Dawson JD, Riley WA, Lauer RM. Carotid intimal-medial thickness is related to cardiovascular risk factors measured from childhood through middle age: The Muscatine Study. Circulation 2001;104:2815-9.

71. Li S, Chen W, Srinivasan SR, et al. Childhood cardiovascular risk factors and carotid vascular changes in adulthood: the Bogalusa Heart Study. Jama 2003;290:2271-6.

72. Chen X, Wang Y. Tracking of blood pressure from childhood to adulthood: a systematic review and meta-regression analysis. Circulation 2008;117:3171-80.

73. Hanevold C, Waller J, Daniels S, Portman R, Sorof J, International Pediatric Hypertension A. The effects of obesity, gender, and ethnic group on left ventricular hypertrophy and geometry in hypertensive children: a collaborative study of the International Pediatric Hypertension Association. Pediatrics 2004;113:328-33.

74. Lande MB, Carson NL, Roy J, Meagher CC. Effects of childhood primary hypertension on carotid intima media thickness: a matched controlled study. Hypertension 2006;48:40-4. 
75. Brady TM, Fivush B, Flynn JT, Parekh R. Ability of blood pressure to predict left ventricular hypertrophy in children with primary hypertension. The Journal of pediatrics $2008 ; 152: 73-8,8$ e1.

76. Webber LS, Srinivasan SR, Wattigney WA, Berenson GS. Tracking of serum lipids and lipoproteins from childhood to adulthood. The Bogalusa Heart Study. American journal of epidemiology 1991;133:884-99.

77. Morrison JA, Glueck CJ, Woo JG, Wang P. Risk factors for cardiovascular disease and type 2 diabetes retained from childhood to adulthood predict adult outcomes: the Princeton LRC Followup Study. International journal of pediatric endocrinology 2012;2012:6.

78. Magnussen CG, Raitakari OT, Thomson R, et al. Utility of currently recommended pediatric dyslipidemia classifications in predicting dyslipidemia in adulthood: evidence from the Childhood Determinants of Adult Health (CDAH) study, Cardiovascular Risk in Young Finns Study, and Bogalusa Heart Study. Circulation 2008;117:32-42.

79. Freedman DS, Mei Z, Srinivasan SR, Berenson GS, Dietz WH. Cardiovascular risk factors and excess adiposity among overweight children and adolescents: the Bogalusa Heart Study. J Pediatr 2007; 150:12-7 e2.

80. Teixeira PJ, Sardinha LB, Going SB, Lohman TG. Total and regional fat and serum cardiovascular disease risk factors in lean and obese children and adolescents. Obes Res 2001;9:432-42.

81. Celermajer DS, Ayer JG. Childhood risk factors for adult cardiovascular disease and primary prevention in childhood. Heart 2006;92:1701-6.

82. USDHHS. The Surgeon General's call

to action to prevent and decrease overweight and obesity. [Rockville, MD]: U.S. Department of Health and Human Services, Public Health Service, Office of the Surgeon General; [2001].

Available from: U.S. GPO, Washington. Available at http://www.surgeongeneral.gov/library. W V Med J 2001;98:234-43.

83. Deshmukh-Taskar P, Nicklas TA, Morales M, Yang SJ, Zakeri I, Berenson GS. Tracking of overweight status from childhood to young adulthood: the Bogalusa Heart Study. European journal of clinical nutrition 2006;60:48-57.

84. Serdula MK, Ivery D, Coates RJ, Freedman DS, Williamson DF, Byers T. Do obese children become obese adults? A review of the literature. Preventive medicine 1993;22:167-77.

85. Zalesin KC, Franklin BA, Miller WM, Peterson ED, McCullough PA. Impact of obesity on cardiovascular disease. The Medical clinics of North America 2011;95:919-37.

86. Eckel RH, Barouch WW, Ershow AG. Report of the National Heart, Lung, and Blood Institute-National Institute of Diabetes and Digestive and Kidney Diseases Working Group on the pathophysiology of obesity-associated cardiovascular disease. Circulation 2002;105:2923-8.

87. Must A, Spadano J, Coakley EH, Field AE, Colditz G, Dietz WH. The disease burden associated with overweight and obesity. Jama 1999;282:1523-9.

88. Krauss RM, Winston M, Fletcher BJ, Grundy SM. Obesity : impact on cardiovascular disease. Circulation 1998;98:1472-6.

89. Kotchen TA. Obesity-related hypertension: epidemiology, pathophysiology, and clinical management. American journal of hypertension 2010;23:1170-8.

90. Ogden CL, Carroll MD, Kit BK, Flegal KM. Prevalence of childhood and adult obesity in the United States, 2011-2012. Jama 2014;311:806-14.

91. NCEP. Third Report of the National Cholesterol Education Program (NCEP) Expert Panel on Detection, Evaluation, and Treatment of High Blood Cholesterol in Adults (Adult Treatment Panel III) final report. Circulation 2002;106:3143-421.

92. Malin G, Morris R, Riley R, Teune M, Khan K. When is birthweight at term ( $>/=37$ weeks' gestation) abnormally low? A systematic review and meta-analysis of the prognostic and predictive ability of current birthweight standards for childhood and adult outcomes. BJOG : an international journal of obstetrics and gynaecology 2015. 
93. Virani SS. Non-HDL cholesterol as a metric of good quality of care: opportunities and challenges. Tex Heart Inst J 2011;38:160-2.

94. Emerging Risk Factors C, Di Angelantonio E, Sarwar N, et al. Major lipids, apolipoproteins, and risk of vascular disease. JAMA 2009;302:1993-2000. 


\section{Tables}

\section{Chapter 2}

Table 1: Maternal and child characteristics at birth and in fifth grade using merged data from the Birth Score Project (1994-2000) and the CARDIAC Project (2004-2010) for all infants who were born Full-Term $(\mathrm{N}=19583)$

\begin{tabular}{|c|c|c|c|}
\hline Variable & $\mathbf{N}$ & Missing & $\begin{array}{l}\text { Frequency }(\%) / \\
\text { Mean (SD) }\end{array}$ \\
\hline Sex & 19583 & 0 & \\
\hline Female & & & $10713(54.71)$ \\
\hline Male & & & $8870(45.29)$ \\
\hline Race (child in fifth grade) & 19049 & 534 & \\
\hline Others & & & $931(4.89)$ \\
\hline White & & & $18118(95.11)$ \\
\hline Race (mother at birth) & 19583 & 0 & \\
\hline Others & & & $482(2.46)$ \\
\hline White & & & $19101(97.54)$ \\
\hline Marital Status (at birth) & 19583 & 0 & \\
\hline Single & & & $5072(25.9)$ \\
\hline Married & & & $14511(74.1)$ \\
\hline Health Insurance & 10657 & 8926 & \\
\hline Medicaid & & & $5937(55.71)$ \\
\hline Non-Medicaid & & & $4720(44.29)$ \\
\hline Maternal Education at Birth & 19516 & 67 & \\
\hline Less than or equal to 12 years & & & $12642(64.78)$ \\
\hline Greater than 12 years & & & $6874(35.22)$ \\
\hline Maternal Education when child in 5 grade & 17820 & 1763 & \\
\hline Less than or equal to 12 years & & & $7769(43.6)$ \\
\hline Greater than 12 years & & & $10051(56.4)$ \\
\hline Family History of Cholesterol & 15781 & 3802 & \\
\hline No & & & $10055(63.72)$ \\
\hline Yes & & & $5726(36.28)$ \\
\hline Family History of CVD & 19583 & 0 & \\
\hline No & & & $13098(66.88)$ \\
\hline Yes & & & $6485(33.12)$ \\
\hline Family History of Diabetes & 17897 & 1686 & \\
\hline No & & & $8513(47.57)$ \\
\hline Yes & & & $9384(52.43)$ \\
\hline Smoking during Pregnancy (maternal) & 19428 & 155 & \\
\hline Yes & & & $4839(24.91)$ \\
\hline No & & & $14590(75.09)$ \\
\hline Smoking in the house when child in 5 grade & 18564 & 1019 & \\
\hline
\end{tabular}




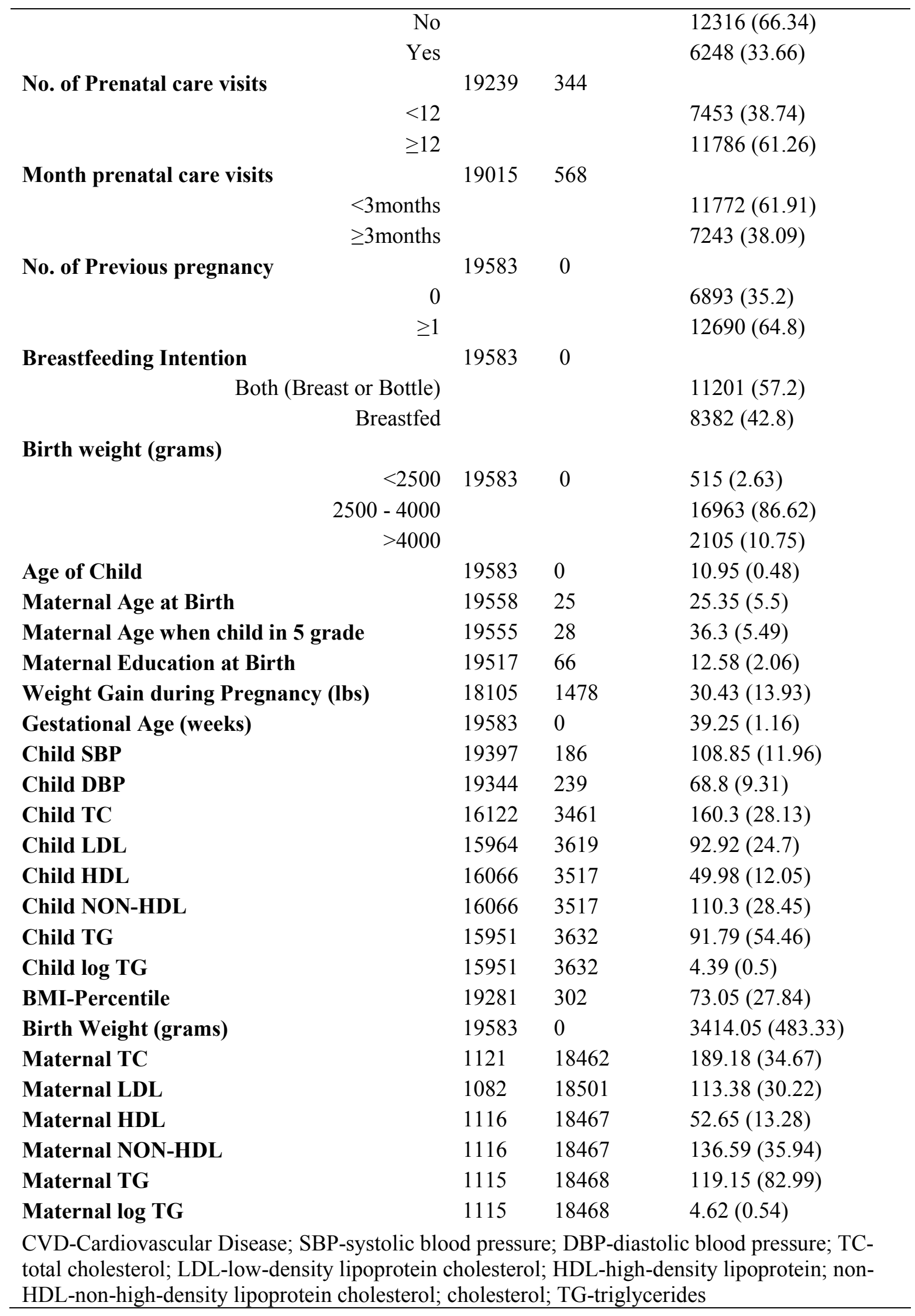


Table 2: Results one-way analysis of variance (ANOVA) for the mean difference in blood pressure and lipid levels in both fifth grade children and mothers by low birth weight $(<2500 \mathrm{~g})$ normal birth weight $(2000-4000 \mathrm{~g})$ and high birth weight $(>4000 \mathrm{~g})$ groups using merged data from the Birth Score Project (1994-2000) and the CARDIAC Project (2004-2010) for all infants who were born Full-Term ( $\mathrm{N}=19583)$

\begin{tabular}{|c|c|c|c|c|c|c|}
\hline \multirow{4}{*}{$\begin{array}{l}\text { Birth weight } \\
\text { categories } \\
\text { (grams) } \\
\text { BMI (N) } \\
\text { Mean (SD)* }\end{array}$} & \multicolumn{3}{|c|}{ Child CVD risk factors } & \multicolumn{3}{|c|}{ Mother CVD risk factors } \\
\hline & $>2500$ & $2500-4000$ & $>4000$ & $>2500$ & $\begin{array}{c}2500- \\
4000\end{array}$ & $>4000$ \\
\hline & 504 & 16707 & 2070 & & & \\
\hline & $\begin{array}{c}66.04 \\
(30.64)\end{array}$ & $\begin{array}{c}72.51 \\
(28.07)\end{array}$ & $\begin{array}{c}79.14 \\
(24.13)\end{array}$ & & & \\
\hline $\begin{array}{l}\text { SBP (N) } \\
\text { Mean (SD)* }\end{array}$ & $\begin{array}{c}510 \\
\mathbf{1 0 8 . 3} \\
\mathbf{( 1 1 . 7 5 )}\end{array}$ & $\begin{array}{r}16800 \\
\mathbf{1 0 8 . 7 9} \\
\mathbf{( 1 1 . 9 8 )}\end{array}$ & $\begin{array}{c}2087 \\
\mathbf{1 0 9 . 5 8} \\
(\mathbf{1 1 . 8 9 )}\end{array}$ & & & \\
\hline $\begin{array}{l}\operatorname{DBP}(\mathbf{N}) \\
\operatorname{Mean}(\mathrm{SD}) *\end{array}$ & $\begin{array}{c}509 \\
\mathbf{6 8 . 4 4} \\
(\mathbf{9 . 1 4})\end{array}$ & $\begin{array}{l}16754 \\
\mathbf{6 8 . 7 4} \\
\mathbf{( 9 . 3 4 )}\end{array}$ & $\begin{array}{l}2081 \\
\mathbf{6 9 . 3 6} \\
(\mathbf{9 . 1 4 )}\end{array}$ & & & \\
\hline $\begin{array}{l}\text { TC }(\mathrm{N}) \\
\text { Mean (SD) }\end{array}$ & $\begin{array}{c}437 \\
161.33 \\
(30.21)\end{array}$ & $\begin{array}{c}13982 \\
160.21 \\
(28.18)\end{array}$ & $\begin{array}{c}1703 \\
160.8 \\
(27.17)\end{array}$ & $\begin{array}{c}26 \\
188.74 \\
(28.92)\end{array}$ & $\begin{array}{c}970 \\
188.52 \\
(35.27)\end{array}$ & $\begin{array}{c}120 \\
194.71 \\
(30.47)\end{array}$ \\
\hline $\begin{array}{l}\text { LDL (N) } \\
\text { Mean (SD) }\end{array}$ & $\begin{array}{c}433 \\
94.08 \\
(26.63)\end{array}$ & $\begin{array}{c}13844 \\
92.79 \\
(24.63)\end{array}$ & $\begin{array}{c}1687 \\
93.74 \\
(24.81)\end{array}$ & $\begin{array}{c}26 \\
188.74 \\
(28.92)\end{array}$ & $\begin{array}{c}970 \\
188.52 \\
(35.27)\end{array}$ & $\begin{array}{c}120 \\
194.71 \\
(30.47)\end{array}$ \\
\hline $\begin{array}{l}\text { HDL (N) } \\
\text { Mean (SD) }\end{array}$ & $\begin{array}{c}436 \\
49.61 \\
(11.99)\end{array}$ & $\begin{array}{c}13933 \\
49.97 \\
(12.08)\end{array}$ & $\begin{array}{c}1697 \\
50.18 \\
(11.89)\end{array}$ & $\begin{array}{c}25 \\
51.4 \\
(11.55)\end{array}$ & $\begin{array}{c}970 \\
52.79 \\
(13.41)\end{array}$ & $\begin{array}{c}121 \\
51.82 \\
(12.61)\end{array}$ \\
\hline $\begin{array}{l}\text { Non-HDL (N) } \\
\text { Mean (SD) }\end{array}$ & $\begin{array}{c}436 \\
111.66 \\
(29.62)\end{array}$ & $\begin{array}{c}13933 \\
110.23 \\
(28.47)\end{array}$ & $\begin{array}{c}1697 \\
110.58 \\
(27.97)\end{array}$ & $\begin{array}{c}25 \\
138.4 \\
(30.15)\end{array}$ & $\begin{array}{c}970 \\
135.84 \\
(36.63)\end{array}$ & $\begin{array}{c}121 \\
142.2 \\
(30.85)\end{array}$ \\
\hline $\begin{array}{l}\log -T G(\mathbf{N}) \\
\operatorname{Mean}(\mathrm{SD})\end{array}$ & $\begin{array}{c}432 \\
4.4 \\
(0.52)\end{array}$ & $\begin{array}{c}13841 \\
4.39 \\
(0.5)\end{array}$ & $\begin{array}{c}1678 \\
4.36 \\
(0.51)\end{array}$ & $\begin{array}{c}25 \\
4.57 \\
(0.51)\end{array}$ & $\begin{array}{c}969 \\
4.62 \\
(0.54)\end{array}$ & $\begin{array}{c}121 \\
4.67 \\
(0.58)\end{array}$ \\
\hline
\end{tabular}

$* \mathrm{P}<0.05$ (omnibus F-test)

Post hoc test (Tukeys) were performed where the omnibus F-test was significant to explore which means were significantly different between groups. All comparisons were significant for BMI. For SBP and DBP only high birth weigh $(>4000 \mathrm{~g})$ was significantly greater than normal birth weigh $(2500-4000 \mathrm{~g})(\mathrm{p}<0.05)$

CVD-Cardiovascular Disease; SBP-systolic blood pressure; DBP-diastolic blood pressure; TCtotal cholesterol; LDL-low-density lipoprotein cholesterol; HDL-high-density lipoprotein; nonHDL-non-high-density lipoprotein cholesterol; cholesterol; TG-triglycerides 
Table 3: Pearson correlation between birth weight and CVD risk factors for mothers and children using merged data from the Birth Score Project (1994-2000) and the CARDIAC Project (20042010) for all infants who were born Full-Term $(\mathrm{N}=19,583)$

\begin{tabular}{cccc}
\hline Childhood CVD risk factors & N & Correlation Coefficient $\mathbf{r}$ & P-value \\
\hline BMI percentile & 19281 & $\mathbf{0 . 1 0 2 8 4}$ & $<0.0001$ \\
SBP & 19397 & $\mathbf{0 . 0 2 6 4 7}$ & 0.0002 \\
DBP & 19344 & $\mathbf{0 . 0 3 2 2 4}$ & $<0.0001$ \\
TC & 16122 & 0.00568 & 0.4704 \\
LDL & 15964 & 0.00336 & 0.6709 \\
HDL & 16066 & $\mathbf{0 . 0 2 3 0 5}$ & 0.0035 \\
Non-HDL & 16066 & -0.00565 & 0.4742 \\
Log-TG & 15951 & $\mathbf{0 . 0 2 7 6 8}$ & 0.0005 \\
\hline
\end{tabular}

Maternal CVD risk factors

\begin{tabular}{cccc}
\hline TC & 1116 & $\mathbf{0 . 0 6 1 9 3}$ & 0.0386 \\
LDL & 1082 & 0.04892 & 0.1078 \\
HDL & 1116 & -0.00539 & 0.8574 \\
Non-HDL & 1116 & 0.04932 & 0.0996 \\
Log-TG & 1115 & 0.03763 & 0.2093
\end{tabular}

CVD-Cardiovascular Disease; SBP-systolic blood pressure; DBP-diastolic blood pressure; TCtotal cholesterol; LDL-low-density lipoprotein cholesterol; HDL-high-density lipoprotein; nonHDL-non-high-density lipoprotein cholesterol; cholesterol; TG-triglycerides 
Table 4a: Results of the multiple regression analysis for the association between birth weight and CVD risk factors of fifth-grade WV children using merged data from the Birth Score Project (1994-2000) and the CARDIAC Project (2004-2010) for all infants who were born Full-Term $(\mathrm{N}=19,583)$

\begin{tabular}{|c|c|c|c|c|c|c|c|c|c|}
\hline & \multicolumn{3}{|c|}{ Model 1} & \multicolumn{3}{|c|}{ Model 2} & \multicolumn{3}{|c|}{ Model 3} \\
\hline & $\mathbf{N}$ & $\begin{array}{c}\text { Unstandardized } \\
\text { regression } \\
\text { coefficients }(95 \% \\
\text { CI) }\end{array}$ & $\begin{array}{l}\text { Standardiz } \\
\text { ed (Beta) }\end{array}$ & $\mathbf{N}$ & $\begin{array}{c}\text { Unstandardized } \\
\text { regression } \\
\text { coefficients }(95 \% \\
\text { CI) }\end{array}$ & $\begin{array}{l}\text { Standardiz } \\
\text { ed (Beta) }\end{array}$ & $\mathbf{N}$ & $\begin{array}{c}\text { Unstandardized } \\
\text { regression } \\
\text { coefficients }(95 \% \\
\text { CI) }\end{array}$ & $\begin{array}{c}\text { Standar } \\
\text { dized } \\
\text { (Beta) }\end{array}$ \\
\hline SBP & 1939 & 0.0007 & 0.026 & 1911 & -0.0002 & -0.009 & 8289 & -0.0002 & -0.007 \\
\hline Birth weight & 7 & $(0.0003-0.001)^{* *}$ & & 3 & $(-0.0005-0.0001)$ & & & $(-0.0006-0.0003)$ & \\
\hline LBW & & $\begin{array}{c}-0.49 \\
(-1.54-0.56)\end{array}$ & -0.007 & & $\begin{array}{c}0.55 \\
(-0.44-1.56)\end{array}$ & 0.007 & & $\begin{array}{c}0.48 \\
(-0.91-1.86)\end{array}$ & 0.007 \\
\hline HBW & & $\begin{array}{c}0.80 \\
(0.25-1.34) * *\end{array}$ & 0.021 & & $\begin{array}{c}-0.13 \\
(-0.64-0.39)\end{array}$ & -0.003 & & $\begin{array}{c}0.05 \\
(-0.71-0.82)\end{array}$ & 0.001 \\
\hline DBP & 1934 & 0.0006 & 0.032 & 1906 & 0.00009 & 0.005 & 1501 & 0.00010 & 0.005 \\
\hline $\begin{array}{l}\text { Birth weight } \\
\text { LBW }\end{array}$ & 4 & $\begin{array}{c}(\mathbf{0 . 0 0 0 3}-\mathbf{0 . 0 0 0 8}) * * \\
-0.31 \\
(-1.13-0.52)\end{array}$ & -0.005 & 3 & $\begin{array}{c}(-0.0001-0.0004) \\
0.35 \\
(-0.45-1.15)\end{array}$ & 0.006 & 8 & $\begin{array}{c}(-0.0002-0.0004) \\
0.38 \\
(-0.52-1.29)\end{array}$ & 0.007 \\
\hline HBW & & $\begin{array}{c}0.62 \\
(0.19-1.04) * *\end{array}$ & 0.021 & & $\begin{array}{c}0.034 \\
(-0.38-0.45)\end{array}$ & 0.001 & & $\begin{array}{c}0.13 \\
(-0.33-0.59)\end{array}$ & 0.004 \\
\hline TC & 1612 & 0.0003 & 0.006 & 1606 & -0.0002 & -0.004 & 1327 & -0.0007 & -0.013 \\
\hline Birth weight & 2 & $\begin{array}{c}(-0.0006-0.001) \\
1.12\end{array}$ & 0.006 & 6 & $\begin{array}{c}(-0.001-0.0007) \\
1.83\end{array}$ & 0.011 & 1 & $\begin{array}{c}(-0.0017-0.0002) \\
1.71\end{array}$ & 0.01 \\
\hline LBW & & $\begin{array}{c}(-1.56-3.80) \\
0.59 \\
(-0.82-2.00)\end{array}$ & 0.006 & & $\begin{array}{c}(-0.84-4.49) \\
-0.02 \\
(-1.44-1.39)\end{array}$ & 0 & & $\begin{array}{c}(-1.23-4.64) \\
-0.36 \\
(-1.90-1.19)\end{array}$ & -0.004 \\
\hline $\begin{array}{l}\text { LDL } \\
\text { Birth weight }\end{array}$ & $\begin{array}{c}1596 \\
4\end{array}$ & $\begin{array}{c}0.0002 \\
(-0.0006-0.001)\end{array}$ & 0.003 & $\begin{array}{c}1590 \\
8\end{array}$ & $\begin{array}{c}-0.0006 \\
(-0.001-0.0002)\end{array}$ & -0.011 & $\begin{array}{c}1313 \\
9\end{array}$ & $\begin{array}{l}-0.0010 \\
(-0.0019-- \\
0.00016) *\end{array}$ & -0.02 \\
\hline LBW & & $\begin{array}{c}1.29 \\
(-1.08-3.65)\end{array}$ & 0.008 & & $\begin{array}{c}2.20 \\
(-0.14-4.54)\end{array}$ & 0.014 & & $\begin{array}{c}2.61 \\
(0.05-5.18)^{*}\end{array}$ & 0.017 \\
\hline
\end{tabular}




\begin{tabular}{|c|c|c|c|c|c|c|c|c|c|}
\hline HBW & & $\begin{array}{c}0.95 \\
(-0.30-2.2)\end{array}$ & 0.012 & & $\begin{array}{c}0.10 \\
(-1.14-1.34)\end{array}$ & 0.001 & & $\begin{array}{c}0.08 \\
(-1.27-1.43)\end{array}$ & 0.001 \\
\hline HDL & 1606 & 0.0006 & 0.023 & 1601 & 0.0014 & 0.057 & 1549 & 0.0009 & 0.036 \\
\hline Birth weight & 6 & $(0.0002-0.0009)^{* * *}$ & & 0 & $(0.0011-0.0018)^{* * *}$ & & 1 & $(0.0005-0.0012) *$ & \\
\hline LBW & & $\begin{array}{c}-0.36 \\
(-1.50-0.79)\end{array}$ & -0.005 & & $\begin{array}{c}-1.25 \\
(-2.34--0.17) *\end{array}$ & -0.017 & & $\begin{array}{c}-0.85 \\
(-1.95-0.26)\end{array}$ & -0.011 \\
\hline HBW & & $\begin{array}{c}0.21 \\
(-0.39-0.82)\end{array}$ & 0.005 & & $\begin{array}{c}1.19 \\
(0.62-1.77) * *\end{array}$ & 0.03 & & $\begin{array}{c}0.71 \\
(0.12-1.30) *\end{array}$ & 0.018 \\
\hline Non-HDL & 1606 & -0.0003 & -0.006 & 1601 & -0.0017 & -0.029 & 1541 & -0.0018 & -0.032 \\
\hline Birth weight & 6 & $(-0.001-0.0006)$ & & 0 & $\begin{array}{l}(-0.0026-- \\
0.0008) * *\end{array}$ & & 9 & $\begin{array}{l}(-0.0028-- \\
0.0009) * *\end{array}$ & \\
\hline LBW & & $\begin{array}{c}1.43 \\
(-1.28-4.15)\end{array}$ & 0.008 & & $\begin{array}{c}3.02 \\
(0.38-5.67) *\end{array}$ & 0.017 & & $\begin{array}{c}3.18 \\
(0.27-6.08) *\end{array}$ & 0.018 \\
\hline HBW & & $\begin{array}{c}0.35 \\
(-1.08-1.78)\end{array}$ & 0.004 & & $\begin{array}{c}-1.25 \\
(-2.65-0.16)\end{array}$ & -0.013 & & $\begin{array}{c}-1.07 \\
(-2.60-0.45)\end{array}$ & -0.012 \\
\hline Log-TG & 1595 & -0.00003 & -0.028 & 1589 & -0.00007 & -0.064 & 1277 & -0.00005 & -0.048 \\
\hline Birth weight & 1 & $\begin{array}{c}(-0.00004- \\
-0.00001)^{* *}\end{array}$ & & 6 & $\begin{array}{l}(-0.00008- \\
-0.00005)^{* *}\end{array}$ & & 6 & $\begin{array}{l}(-0.00006-- \\
0.00003)^{* *}\end{array}$ & \\
\hline LBW & & $\begin{array}{c}0.014 \\
(-0.033-0.062)\end{array}$ & 0.005 & & $\begin{array}{c}0.054 \\
(0.009-0.099)^{*}\end{array}$ & 0.018 & & $\begin{array}{c}0.046 \\
(-0.003-0.097)\end{array}$ & 0.015 \\
\hline HBW & & $\begin{array}{c}-0.02 \\
(-0.05-0.005)\end{array}$ & -0.013 & & $\begin{array}{c}-0.065 \\
(-0.089--0.042)^{* *}\end{array}$ & -0.04 & & $\begin{array}{c}-0.05 \\
(-0.08--0.02) *\end{array}$ & -0.031 \\
\hline
\end{tabular}

$*<0.05 ; * *<0.01$

LBW (<2,500 grams), HBW ( $>4000$ grams), Referent: normal birth weight (2500-4000grams).

Variables included in the model: Model 1: All the outcomes were regressed on birth weight (both linear and categorical). Model 2: All the outcomes were regressed on birth weight variable and the child's BMI percentile in fifth grade. Model 3: All the outcomes were regressed on birth weight and the child's BMI percentile and additional covariates. Only covariates that were significant in the Spearman's correlation were used in the multiple regression analysis. Each non-significant covariate was deleted from the regression model one at a time. Variables retained in model for SBP: child's age, race (white vs. other), maternal health insurance status at time of delivery (non-Medicaid vs. Medicaid), and family history of cholesterol (yes vs. no); DBP: child's age, race (white vs. other), and family history of cholesterol (yes vs. no); TC: child's age, race (white vs. other), and family history of cholesterol (yes vs. no); LDL: child's age, gender, interaction between age*gender, and family history of cholesterol (yes vs. no); HDL: child's age, gender, race (white vs. other), family history of CVD (yes vs. no), maternal smoking status during pregnancy (yes vs. no), and maternal age at time of delivery; Non-HDL: child's age, family history of cholesterol (yes vs. no), and breastfeeding intention 
(breastfeed vs. both); TG: child's age, sex, race (white vs. other), family history of cholesterol (yes vs. no), family history of CVD (yes vs. no), and maternal education at birth.

CVD-Cardiovascular Disease; SBP-systolic blood pressure; DBP-diastolic blood pressure; TC-total cholesterol; LDL-low-density lipoprotein cholesterol; HDL-high-density lipoprotein; non-HDL-non-high-density lipoprotein cholesterol; cholesterol; TG-triglycerides 
Table 4b: Table 4a: Results of the multiple regression analysis for the association between birth weight and CVD risk factors of mothers using merged data from the Birth Score Project (1994-2000) and the CARDIAC Project (2004-2010) for all infants who were born Full-Term $(\mathrm{N}=19583)$

\begin{tabular}{|c|c|c|c|c|c|c|c|c|c|c|}
\hline & \multicolumn{5}{|c|}{ Model 1} & \multicolumn{5}{|c|}{ Model 2} \\
\hline & $\mathbf{N}$ & $\begin{array}{c}\text { Unstandardized } \\
\text { regression coefficients } \\
(95 \% \mathrm{CI})\end{array}$ & $\begin{array}{l}\text { Standa } \\
\text { rdized } \\
\text { (Beta) }\end{array}$ & $\begin{array}{c}\text { P- } \\
\text { Value }\end{array}$ & Adj-R ${ }^{2}$ & $\mathbf{N}$ & $\begin{array}{c}\text { Unstandardized } \\
\text { regression } \\
\text { coefficients }(95 \% \mathrm{CI})\end{array}$ & $\begin{array}{l}\text { Standar } \\
\text { dized } \\
\text { (Beta) }\end{array}$ & $\begin{array}{c}\text { P- } \\
\text { Value }\end{array}$ & Adj-R ${ }^{2}$ \\
\hline $\begin{array}{l}\text { TC } \\
\text { Birth weight }\end{array}$ & 1116 & $\begin{array}{c}0.005 \\
(0.0002-0.00904)\end{array}$ & 0.062 & 0.039 & 0.0029 & 1115 & $\begin{array}{c}0.004 \\
(-0.0001-0.0087)\end{array}$ & 0.057 & 0.0553 & 0.0246 \\
\hline LBW & & $\begin{array}{c}0.22 \\
(-13.29-13.73)\end{array}$ & 0.001 & 0.975 & 0.0013 & & $\begin{array}{c}-0.87 \\
(-14.25-12.50)\end{array}$ & -0.004 & 0.898 & 0.0231 \\
\hline HBW & & $\begin{array}{c}6.19 \\
(-0.38-12.77)\end{array}$ & 0.055 & 0.065 & & & $\begin{array}{c}5.74 \\
(-0.78-12.27)\end{array}$ & 0.051 & 0.0845 & \\
\hline $\begin{array}{l}\text { LDL } \\
\text { Birth weight }\end{array}$ & 1082 & $\begin{array}{c}0.003 \\
(-0.0007-0.007)\end{array}$ & 0.049 & 0.108 & 0.1078 & 1060 & $\begin{array}{c}0.003 \\
(-0.0007-0.0072)\end{array}$ & 0.050 & 0.1019 & 0.0191 \\
\hline LBW & & $\begin{array}{c}3.69 \\
(-8.56-15.94)\end{array}$ & 0.018 & 0.555 & 0.0014 & & $\begin{array}{c}1.602 \\
(-10.74-13.94)\end{array}$ & 0.008 & 0.7989 & 0.0188 \\
\hline HBW & & $\begin{array}{c}5.39 \\
(-0.46-11.25)\end{array}$ & 0.055 & 0.071 & & & $\begin{array}{c}5.49 \\
(-0.36-11.35)\end{array}$ & 0.056 & 0.0662 & \\
\hline $\begin{array}{l}\text { HDL } \\
\text { Birth weight }\end{array}$ & 1116 & $\begin{array}{c}-0.0002 \\
(-0.002-0.002)\end{array}$ & -0.005 & 0.857 & -0.0009 & 1016 & $\begin{array}{c}-0.001 \\
(-0.003-0.0004)\end{array}$ & -0.045 & 0.1444 & 0.0563 \\
\hline LBW & & $\begin{array}{c}-1.38 \\
(-6.66-3.90)\end{array}$ & -0.015 & 0.608 & -0.0011 & & $\begin{array}{c}1.73 \\
(-3.60-7.06)\end{array}$ & 0.019 & 0.5252 & 0.0551 \\
\hline HBW & & $\begin{array}{c}-0.96 \\
(-3.48-1.55)\end{array}$ & -0.023 & 0.453 & & & $\begin{array}{c}-1.55 \\
(-4.09-0.98)\end{array}$ & -0.037 & 0.2297 & \\
\hline $\begin{array}{l}\text { Non-HDL } \\
\text { Birth weight }\end{array}$ & 1116 & $\begin{array}{c}0.004 \\
(-0.001-0.008)\end{array}$ & 0.049 & 0.100 & 0.0015 & 1093 & $\begin{array}{c}0.00426 \\
(-0.0003-0.0088)\end{array}$ & 0.054 & 0.07 & 0.0226 \\
\hline LBW & & $\begin{array}{c}2.56 \\
(-11.71-16.83) \\
\end{array}$ & 0.011 & 0.725 & 0.0013 & & $\begin{array}{c}0.48 \\
(-14.20-15.17)\end{array}$ & 0.002 & 0.9486 & 0.0221 \\
\hline
\end{tabular}




\begin{tabular}{|c|c|c|c|c|c|c|c|c|c|c|}
\hline HBW & & $\begin{array}{c}6.35 \\
(-0.44-13.14)\end{array}$ & 0.055 & 0.067 & & & $\begin{array}{c}6.66 \\
(-0.156-13.48)\end{array}$ & 0.058 & 0.0555 & \\
\hline $\begin{array}{l}\text { Log-TG } \\
\text { Birth weight }\end{array}$ & 1115 & $\begin{array}{c}0.00004 \\
(-0.00002-0.0001)\end{array}$ & 0.038 & 0.209 & 0.0005 & 1114 & $\begin{array}{c}0.00005 \\
(-0.000017-0.00012)\end{array}$ & 0.044 & 0.139 & 0.005 \\
\hline LBW & & $\begin{array}{c}-0.06 \\
(-0.27-0.16)\end{array}$ & -0.016 & 0.604 & -0.0008 & & $\begin{array}{c}-0.062 \\
(-0.275-0.151)\end{array}$ & -0.017 & 0.5675 & 0.0034 \\
\hline HBW & & $\begin{array}{c}0.04 \\
(-0.06-0.15)\end{array}$ & 0.026 & 0.391 & & & $\begin{array}{c}0.051 \\
(-0.049-0.153)\end{array}$ & 0.030 & 0.3171 & \\
\hline
\end{tabular}

LBW (<2,500 grams), HBW ( $>4000$ grams), Referent: normal birth weight (2500-4000grams).

Variables included in the model: Model 1: All the outcomes were regressed on birth weight (both linear and categorical). Model 2: All the outcomes were regressed on birth weight and additional covariates. Only covariates that were significant in the Spearman's correlation were used in the multiple regression analysis. Each non-significant covariate was deleted from the regression model one at a time. Variables retained in model for TC: mothers age when child in fifth grade, and breastfeeding intention (breastfeed vs. both); LDL: mothers age when child in fifth grade, breastfeeding intention (breastfeed vs. both), and smoking in the house (yes vs. no); HDL: mothers age when child in fifth grade, smoking in the house (yes vs. no), family history of CVD (yes vs. no), family history of diabetes (yes vs. no), number of previous pregnancies ( $\geq 1$ vs. 0 ); Non-HDL: mothers age when child in fifth grade, breastfeeding intention (breastfeed vs. both), and smoking in the house (yes vs. no); TG: mothers age when child in fifth grade and breastfeeding intention (breastfeed vs. both).

CVD-Cardiovascular Disease; SBP-systolic blood pressure; DBP-diastolic blood pressure; TC-total cholesterol; LDL-low-density lipoprotein cholesterol; HDL-high-density lipoprotein; non-HDL-non-high-density lipoprotein cholesterol; cholesterol; TG-triglycerides 
Table 5a: Results of the multiple regression analysis for the association between birth weight (continuous) and LDL, HDL, non-HDL, and TG levels of fifth-grade WV children born full term using merged data from the Birth Score Project (1994-2000) and the CARDIAC Project (20042010) for all infants who were born Full-Term ( $\mathrm{N}=19583)$

\begin{tabular}{|c|c|c|c|c|c|c|c|c|c|}
\hline & & $\mathbf{N}$ & $\begin{array}{l}\text { Unstandardized } \\
\text { regression coefficients } \\
\text { and } 95 \% \text { CI }\end{array}$ & $\begin{array}{l}\text { Standardiz } \\
\text { ed (Beta) }\end{array}$ & $\begin{array}{c}\mathbf{t} \\
\text { Value }\end{array}$ & $\begin{array}{c}P- \\
\text { value }\end{array}$ & $\begin{array}{c}\text { Adj- } \\
\mathbf{R}^{2}\end{array}$ & $\begin{array}{l}\text { Reduce } \\
\text { d model } \\
\text { Adj-R }^{2}\end{array}$ & $\begin{array}{c}\text { Chan } \\
\text { ge in } \\
\text { Adj- } \\
\mathbf{R}^{2}\end{array}$ \\
\hline \multirow[t]{7}{*}{ LDL } & Intercept & 13139 & $142.72(128.72-156.71)$ & 0 & 19.99 & 0.0001 & $\begin{array}{c}0.036 \\
5\end{array}$ & 0.0361 & 0.0004 \\
\hline & Child's age in fifth grade & & $-5.23(-6.47--3.995)$ & -0.11 & -8.3 & 0.0001 & & & \\
\hline & Sex (male vs. female) & & $-23.32(-42.57--4.07)$ & -0.47 & -2.37 & 0.0176 & & & \\
\hline & Child's sex*age & & $2.25(0.49-3.992)$ & 0.5 & 2.51 & 0.0122 & & & \\
\hline & $\begin{array}{l}\text { Family history of } \\
\text { cholesterol }\end{array}$ & & $5.38(4.52-6.25)$ & 0.11 & 12.21 & 0.0001 & & & \\
\hline & $\begin{array}{l}\text { BMI percentile in fifth } \\
\text { grade }\end{array}$ & & $0.12(0.11-0.14)$ & 0.14 & 15.61 & 0.0001 & & & \\
\hline & Birth weigh (grams) & & $\begin{array}{c}-0.0011(-0.0019-- \\
0.0002)\end{array}$ & -0.02 & -2.33 & 0.0196 & & & \\
\hline \multirow[t]{9}{*}{ HDL } & Intercept & 15491 & $68.8(64.25-73.35)$ & 0 & 29.66 & 0.0001 & 0.124 & 0.1228 & 0.0012 \\
\hline & Child's age in fifth grade & & $-0.97(-1.35--0.59)$ & -0.04 & -5.02 & 0.0001 & & & \\
\hline & Sex (male vs. female) & & $2.18(1.82-2.54)$ & 0.09 & 11.75 & 0.0001 & & & \\
\hline & Race (white vs. other) & & $-2.71(-3.53--1.89)$ & -0.05 & -6.48 & 0.0001 & & & \\
\hline & Family history of CVD & & $-0.75(-1.12--0.38)$ & -0.03 & -3.93 & 0.0001 & & & \\
\hline & $\begin{array}{l}\text { Maternal smoking } \\
\text { (pregnancy) }\end{array}$ & & $-0.74(-1.17--0.32)$ & -0.03 & -3.41 & 0.0007 & & & \\
\hline & Maternal age at birth & & $0.05(0.02-0.09)$ & 0.03 & 2.86 & 0.0042 & & & \\
\hline & $\begin{array}{l}\text { BMI percentile in fifth } \\
\text { grade }\end{array}$ & & $-0.15(-0.16--0.14)$ & -0.34 & -43.96 & 0.0001 & & & \\
\hline & Birth weigh (grams) & & $0.001(0.0006-0.0013)$ & 0.04 & 4.59 & 0.0001 & & & \\
\hline $\begin{array}{l}\text { Non- } \\
\text { HDL }\end{array}$ & Intercept & 13221 & $137.4(125.8-148.99)$ & 0 & 23.22 & 0.0001 & $\begin{array}{c}0.066 \\
8 \\
\end{array}$ & 0.0659 & 0.0009 \\
\hline
\end{tabular}




\begin{tabular}{|c|c|c|c|c|c|c|c|c|c|}
\hline & Child's age in fifth grade & & $-3.57(-4.56--2.57)$ & -0.06 & -7.04 & 0.0001 & & & \\
\hline & $\begin{array}{l}\text { Family history of } \\
\text { cholesterol }\end{array}$ & & $6.45(5.47-7.43)$ & 0.11 & 12.91 & 0.0001 & & & \\
\hline & Feeding intention & & $-0.99(-1.94--0.03)$ & -0.02 & -2.02 & 0.0435 & & & \\
\hline & $\begin{array}{l}\text { BMI percentile in fifth } \\
\text { grade }\end{array}$ & & $0.23(0.22-0.25)$ & 0.23 & 26.31 & 0.0001 & & & \\
\hline & Birth weigh (grams) & & $-0.002(-0.003--0.001)$ & -0.032 & -3.75 & 0.0002 & & & \\
\hline \multirow[t]{9}{*}{ Log-TG } & Intercept & 12776 & $3.7(3.49-3.92)$ & 0 & 33.85 & 0.0001 & $\begin{array}{c}0.142 \\
1\end{array}$ & 0.1401 & 0.002 \\
\hline & Child's age in fifth grade & & $0.04(0.02-0.06)$ & 0.04 & 4.03 & 0.0001 & & & \\
\hline & Sex (male vs. female) & & $-0.11(-0.13--0.1)$ & -0.11 & -12.9 & 0.0001 & & & \\
\hline & Race (white vs. other) & & $0.15(0.11-0.19)$ & 0.07 & 7.67 & 0.0001 & & & \\
\hline & $\begin{array}{l}\text { Family history of } \\
\text { cholesterol }\end{array}$ & & $0.06(0.04-0.08)$ & 0.06 & 6.38 & 0.0001 & & & \\
\hline & Family history of CVD & & $0.02(0.01-0.04)$ & 0.02 & 2.18 & 0.0296 & & & \\
\hline & Maternal education at birth & & $-0.01(-0.02--0.01)$ & -0.04 & -4.18 & 0.0001 & & & \\
\hline & $\begin{array}{l}\text { BMI percentile in fifth } \\
\text { grade }\end{array}$ & & $0.006(0.0059-0.0065)$ & 0.35 & 41.98 & 0.0001 & & & \\
\hline & Birth weigh (grams) & & $\begin{array}{c}-0.00005(-0.00007-- \\
0.00004)\end{array}$ & -0.05 & -5.66 & 0.0001 & & & \\
\hline
\end{tabular}

Only covariates that were significant in the Spearman's correlation were used in the multiple regression analysis. Each non-significant covariate was deleted from the regression model one at a time. Variables retained in model for LDL: child's age, gender, interaction between age*sex, and family history of cholesterol (yes vs. no); HDL: child's age, gender, race (white vs. other), family history of CVD (yes vs. no), maternal smoking status during pregnancy (yes vs. no), and maternal age at time of delivery; Non-HDL: child's age, family history of cholesterol (yes vs. no), and breastfeeding intention (breastfeed vs. both); TG: child's age, sex, race (white vs. other), family history of cholesterol (yes vs. no), family history of CVD (yes vs. no), and maternal education at birth.

CVD-Cardiovascular Disease; LDL-low-density lipoprotein cholesterol; HDL-high-density lipoprotein; non-HDL-non-high-density lipoprotein cholesterol; cholesterol; TG-triglycerides 
Table 5b: Results of the multiple regression analysis for the association between birth weight (categorical) and LDL, HDL, non-HDL, and TG levels of fifth-grade WV children born full term using merged data from the Birth Score Project (1994-2000) and the CARDIAC Project (20042010) for all infants who were born Full-Term ( $\mathrm{N}=19583)$

\begin{tabular}{|c|c|c|c|c|c|c|c|c|c|}
\hline & & $\mathbf{N}$ & $\begin{array}{c}\text { Unstandardized } \\
\text { regression } \\
\text { coefficients and } 95 \% \\
\text { CI } \\
\end{array}$ & $\begin{array}{l}\text { Standardiz } \\
\text { ed (Beta) }\end{array}$ & $\begin{array}{c}\text { t } \\
\text { Value }\end{array}$ & P-value & Adj-R ${ }^{2}$ & $\begin{array}{c}\text { Reduce } \\
\text { d model } \\
\text { Adj-R }\end{array}$ & $\begin{array}{c}\text { Change } \\
\text { in Adj- } \\
\quad \mathbf{R}^{2}\end{array}$ \\
\hline \multirow[t]{8}{*}{ LDL } & Intercept & 13139 & $\begin{array}{c}138.95(125.41- \\
152.49)\end{array}$ & 0 & 20.12 & $<0.0001$ & 0.0363 & 0.0361 & 0.0002 \\
\hline & Child's age in fifth grade & & $-5.21(-6.44--3.97)$ & -0.11 & -8.26 & $<0.0001$ & & & \\
\hline & Sex (male vs. female) & & $-23.54(-42.79--4.29)$ & -0.48 & -2.4 & 0.0166 & & & \\
\hline & Child's sex*age & & $2.25(0.5-4.01)$ & 0.51 & 2.52 & 0.0118 & & & \\
\hline & $\begin{array}{l}\text { Family history of } \\
\text { cholesterol }\end{array}$ & & $5.39(4.53-6.25)$ & 0.11 & 12.23 & $<0.0001$ & & & \\
\hline & $\begin{array}{l}\text { BMI percentile in fifth } \\
\text { grade }\end{array}$ & & $0.12(0.11-0.14)$ & 0.14 & 15.46 & $<0.0001$ & & & \\
\hline & LBW (<2500 gram) & & $2.62(0.05-5.18)$ & 0.02 & 2 & 0.046 & & & \\
\hline & HBW (>4000 grams) & & $0.09(-1.27-1.44)$ & 0.01 & 0.12 & 0.9022 & & & \\
\hline \multirow[t]{10}{*}{ HDL } & Intercept & 15491 & $71.93(67.59-76.27)$ & 0 & 32.5 & $<0.0001$ & 0.1232 & 0.1228 & 0.0004 \\
\hline & Child's age in fifth grade & & $-1(-1.37--0.62)$ & -0.04 & -5.15 & $<0.0001$ & & & \\
\hline & Sex (male vs. female) & & $2.26(1.9-2.62)$ & 0.1 & 12.25 & $<0.0001$ & & & \\
\hline & Race (white vs. other) & & $-2.66(-3.47--1.84)$ & -0.05 & -6.35 & $<0.0001$ & & & \\
\hline & Family history of CVD & & $-0.75(-1.13--0.38)$ & -0.03 & -3.94 & $<0.0001$ & & & \\
\hline & $\begin{array}{l}\text { Maternal smoking } \\
\text { (pregnancy) }\end{array}$ & & $-0.88(-1.3--0.46)$ & -0.04 & -4.09 & $<0.0001$ & & & \\
\hline & Maternal age at birth & & $0.06(0.02-0.09)$ & 0.03 & 3.01 & 0.0026 & & & \\
\hline & $\begin{array}{l}\text { BMI percentile in fifth } \\
\text { grade }\end{array}$ & & $-0.15(-0.15--0.14)$ & -0.34 & -43.77 & $<0.0001$ & & & \\
\hline & LBW $(<2500$ gram $)$ & & $-0.85(-1.96-0.26)$ & -0.02 & -1.5 & 0.1327 & & & \\
\hline & HBW (>4000 grams) & & $0.72(0.13-1.31)$ & 0.02 & 2.37 & 0.0177 & & & \\
\hline
\end{tabular}




\begin{tabular}{|c|c|c|c|c|c|c|c|c|c|}
\hline \multirow[t]{7}{*}{ Non-HDL } & Intercept & 13221 & $\begin{array}{c}130.81(119.82- \\
141.8)\end{array}$ & 0 & 23.33 & $<0.0001$ & 0.0662 & 0.0659 & 0.0003 \\
\hline & Child's age in fifth grade & & $-3.53(-4.52--2.54)$ & -0.06 & -6.97 & $<0.0001$ & & & \\
\hline & $\begin{array}{l}\text { Family history of } \\
\text { cholesterol }\end{array}$ & & $6.46(5.48-7.44)$ & 0.11 & 12.92 & $<0.0001$ & & & \\
\hline & Feeding Intention & & $-1.07(-2.02--0.12)$ & -0.02 & -2.2 & 0.0281 & & & \\
\hline & $\begin{array}{l}\text { BMI Percentile in fifth } \\
\text { grade }\end{array}$ & & $0.23(0.21-0.25)$ & 0.23 & 26.14 & $<0.0001$ & & & \\
\hline & LBW (<2500 gram) & & $3.19(0.28-6.09)$ & 0.02 & 2.15 & 0.0316 & & & \\
\hline & HBW ( $>4000$ grams) & & $-1.08(-2.61-0.46)$ & -0.02 & -1.38 & 0.1671 & & & \\
\hline \multirow[t]{10}{*}{ Log-TG } & Intercept & 12776 & $3.54(3.34-3.75)$ & 0 & 33.5 & $<0.0001$ & 0.1411 & 0.1401 & 0.001 \\
\hline & Child's age in fifth grade & & $0.04(0.02-0.06)$ & 0.04 & 4.17 & $<0.0001$ & & & \\
\hline & Sex (male vs. female) & & $-0.12(-0.13--0.1)$ & -0.12 & -13.4 & $<0.0001$ & & & \\
\hline & Race (white vs. other) & & $0.15(0.11-0.19)$ & 0.07 & 7.52 & $<0.0001$ & & & \\
\hline & $\begin{array}{l}\text { Family history of } \\
\text { cholesterol }\end{array}$ & & $0.06(0.04-0.08)$ & 0.06 & 6.42 & $<0.0001$ & & & \\
\hline & Family history of CVD & & $0.02(0.01-0.04)$ & 0.02 & 2.17 & 0.0302 & & & \\
\hline & Maternal education at birth & & $-0.01(-0.02--0.01)$ & -0.04 & -4.66 & $<0.0001$ & & & \\
\hline & $\begin{array}{l}\text { BMI percentile in fifth } \\
\text { grade }\end{array}$ & & $\begin{array}{c}0.006(0.0059- \\
0.0065)\end{array}$ & 0.35 & 41.8 & $<0.0001$ & & & \\
\hline & LBW (<2500 gram) & & $0.05(-0.01-0.1)$ & 0.02 & 1.82 & 0.0684 & & & \\
\hline & HBW (>4000 grams) & & $-0.06(-0.08--0.03)$ & -0.04 & -3.77 & 0.0002 & & & \\
\hline
\end{tabular}

Only covariates that were significant in the Spearman's correlation were used in the multiple regression analysis. Each non-significant covariate was deleted from the regression model one at a time. Variables retained in model for LDL: child's age, gender, interaction between age*gender, and family history of cholesterol (yes vs. no); HDL: child's age, gender, race (white vs. other), family history of CVD (yes vs. no), maternal smoking status during pregnancy (yes vs. no), and maternal age at time of delivery; Non-HDL: child's age, family history of cholesterol (yes vs. no), and breastfeeding intention (breastfeed vs. both); TG: child's age, sex, race (white vs. other), family history of cholesterol (yes vs. no), family history of CVD (yes vs. no), and maternal education at birth.

CVD-Cardiovascular Disease; LDL-low-density lipoprotein cholesterol; HDL-high-density lipoprotein; non-HDL-non-high-density lipoprotein cholesterol; cholesterol; TG-triglycerides 


\section{Supplemental Tables Chapter 2}

Supplemental Table S1: Results of the Spline regression analysis for the association between birth weight and CVD risk factors of fifth-grade WV children using merged data from the Birth Score Project (1994-2000) and the CARDIAC Project (2004-2010) for all infants who were born Full-Term $(\mathrm{N}=19,583)$

\begin{tabular}{|c|c|c|c|c|c|c|c|c|c|c|}
\hline $\begin{array}{l}\text { CVD risk } \\
\text { factors }\end{array}$ & $\begin{array}{c}\text { BTW } \\
\text { (grams) }\end{array}$ & \multicolumn{3}{|c|}{ Model 1} & \multicolumn{3}{|c|}{ Model 2} & \multicolumn{3}{|c|}{ Model 3} \\
\hline \multirow{2}{*}{ Child } & & $\begin{array}{l}\text { Unstandardized } \\
\text { regression } \\
\text { coefficients and } \\
95 \% \text { CI }\end{array}$ & $\begin{array}{c}\mathrm{P}- \\
\text { value }\end{array}$ & $\begin{array}{c}\text { Adjus } \\
\text { ted- } \\
\mathbf{R}^{2}\end{array}$ & $\begin{array}{c}\text { Unstandardized } \\
\text { regression } \\
\text { coefficients and } \\
95 \% \text { CI }\end{array}$ & $\begin{array}{c}\text { P- } \\
\text { value }\end{array}$ & $\begin{array}{c}\text { Adju } \\
\text { sted- } \\
\mathbf{R}^{2}\end{array}$ & $\begin{array}{c}\text { Unstandardized } \\
\text { regression } \\
\text { coefficients and } \\
95 \% \text { CI }\end{array}$ & $\begin{array}{c}\text { P- } \\
\text { value }\end{array}$ & $\begin{array}{l}\text { Adjus } \\
\text { ted-R }\end{array}$ \\
\hline & & -0.00009 & 0.961 & 0.000 & -0.00105 & 0.543 & 0.115 & 0.00395 & 0.087 & 0.140 \\
\hline \multirow{6}{*}{ SBP } & $<2500$ & $(-0.004-0.004)$ & 6 & 7 & $(-0.0045-0.0023)$ & 7 & 6 & $(-0.0006-0.0085)$ & 4 & 3 \\
\hline & $2500-$ & 0.0006 & 0.771 & & 0.00072 & 0.687 & & -0.0045 & 0.063 & \\
\hline & 4000 & $(-0.0031-0.0043)$ & 2 & & $(-0.0028-0.0042)$ & 1 & & $(-0.009-0.0002)$ & 6 & \\
\hline & & 0.00156 & 0.092 & & 0.00103 & 0.239 & & 0.00157 & 0.224 & \\
\hline & $>4000$ & $(-0.0003-0.0033)$ & 8 & & $(-0.0007-0.0028)$ & 1 & & $(-0.0001-0.004)$ & 5 & \\
\hline & & -0.0009 & 0.512 & & -0.00171 & 0.218 & 0.068 & -0.0008 & 0.628 & 0.073 \\
\hline \multirow[t]{6}{*}{ DBP } & $<2500$ & $(-0.0037-0.0019)$ & 5 & 0.001 & $(-0.004-0.001)$ & 2 & 5 & $(-0.004-0.002)$ & 7 & 2 \\
\hline & $2500-$ & 0.002 & 0.302 & & 0.0018 & & & 0.0009 & & \\
\hline & 4000 & $(-0.001-0.004)$ & 9 & & $(-0.001-0.005)$ & 0.204 & & $(-0.003-0.004)$ & 0.601 & \\
\hline & & 0.0006 & 0.422 & & 0.0002 & 0.752 & & 0.0003 & & \\
\hline & $>4000$ & $(-0.0008-0.002)$ & 2 & & $(-0.0012-0.002)$ & 8 & & $(-0.001-0.002)$ & 0.722 & \\
\hline & & -0.00935 & & 0.000 & -0.0098 & 0.034 & 0.009 & -0.012 & 0.022 & 0.025 \\
\hline \multirow[t]{5}{*}{ TC } & $<2500$ & $(-0.0184-0.00021)$ & 0.045 & 1 & $(-0.019--0.0007)$ & 2 & 3 & $(-0.022--0.0017)$ & 4 & 8 \\
\hline & $2500-$ & 0.01011 & 0.036 & & 0.01008 & 0.036 & & 0.0119 & 0.030 & \\
\hline & 4000 & $(0.00061-0.0196)$ & 8 & & $(0.0006-0.0195)$ & 4 & & $(0.0011-0.02264)$ & 7 & \\
\hline & & -0.0008 & & & -0.0012 & 0.623 & & -0.0012 & & \\
\hline & $>4000$ & $(-0.005-0.004)$ & 0.728 & & $(-0.006-0.004)$ & 5 & & $(-0.006-0.004)$ & 0.649 & \\
\hline LDL & $<2500$ & -0.005 & 0.187 & 0 & -0.006 & 0.137 & 0.021 & -0.009 & 0.044 & 0.036 \\
\hline
\end{tabular}




\begin{tabular}{|c|c|c|c|c|c|c|c|c|c|c|}
\hline & & $(-0.013-0.0026)$ & 4 & & $(-0.014-0.0019)$ & 9 & 7 & $(-0.018--0.0002)$ & 5 & 6 \\
\hline & $2500-$ & 0.0055 & 0.193 & & 0.005 & & & 0.008 & 0.083 & \\
\hline & 4000 & $(-0.0028-0.0139)$ & 4 & & $(-0.002-0.013)$ & 0.195 & & $(-0.001-0.017)$ & 8 & \\
\hline & & 0.00164 & 0.438 & & 0.0012 & & & 0.002 & 0.473 & \\
\hline & $>4000$ & $\left(\begin{array}{lll}-0.00251 & 0.0058)\end{array}\right.$ & 4 & & $(-0.003-0.005)$ & 0.585 & & $(-0.003-0.006)$ & 3 & \\
\hline & & -0.002 & & 0.000 & -0.002 & & 0.110 & -0.0005 & 0.806 & \\
\hline \multirow[t]{7}{*}{ HDL } & $<2500$ & $(-0.006-0.002)$ & 0.369 & 7 & $(-0.005-0.003)$ & 0.527 & 9 & $(-0.004-0.003)$ & 8 & 0.124 \\
\hline & $2500-$ & 0.0028 & 0.184 & & 0.0029 & 0.138 & & 0.0016 & 0.419 & \\
\hline & 4000 & $(-0.0013-0.0068)$ & 9 & & $(-0.0009-0.0067)$ & 4 & & $(-0.0023-0.0054)$ & 5 & \\
\hline & & -0.00225 & & & & & & & & \\
\hline & & $(-0.0043-$ & 0.029 & & -0.0012 & 0.108 & & -0.0013 & 0.190 & \\
\hline & $>4000$ & $0.00023)$ & 1 & & $(-0.003-0.0004)$ & 8 & & $(-0.003-0.0006)$ & 5 & \\
\hline & & -0.007 & 0.123 & & -0.008 & 0.068 & 0.055 & -0.0117 & 0.024 & 0.066 \\
\hline \multirow[t]{7}{*}{ Non-HDL } & $<2500$ & $(-0.016-0.002)$ & 3 & 0 & $(-0.017-0.0007)$ & 9 & 8 & $(-0.022--0.0015)$ & 8 & 9 \\
\hline & $2500-$ & 0.0069 & 0.157 & & 0.00674 & 0.156 & & 0.01007 & 0.063 & \\
\hline & 4000 & $(-0.0027-0.0165)$ & 1 & & $(-0.0026-0.0161)$ & 8 & & $(-0.0006-0.0207)$ & 3 & \\
\hline & & 0.0017 & 0.474 & & 0.0007 & 0.762 & & 0.0008 & 0.748 & \\
\hline & $>4000$ & $(-0.003-0.0065)$ & 3 & & $(-0.0039-0.0054)$ & 5 & & $(-0.004-0.006)$ & 3 & \\
\hline & & -0.00006 & & & -0.00009 & & & -0.0001 & & \\
\hline & & $(-0.00023-$ & 0.450 & 0.000 & $(-0.00024-$ & 0.254 & 0.126 & $(-0.0003-$ & 0.128 & 0.142 \\
\hline \multirow[t]{7}{*}{ Log-TG } & $<2500$ & $0.0001)$ & 8 & 7 & $0.00006)$ & 7 & 4 & $0.00004)$ & 8 & 9 \\
\hline & & 0.00003 & & & 0.00002 & & & 0.00009 & & \\
\hline & $2500-$ & $(-0.00014-$ & 0.745 & & $(-0.00014-$ & 0.804 & & $(-0.00009-$ & 0.331 & \\
\hline & 4000 & $0.0002)$ & 6 & & $0.00018)$ & 1 & & $0.00027)$ & 3 & \\
\hline & & 0.00005 & & & 0.00003 & & & 0.000004 & & \\
\hline & & $(-0.00003-$ & 0.209 & & $(-0.00005-$ & 0.518 & & $(-0.00009-$ & 0.927 & \\
\hline & $>4000$ & $0.00014)$ & 8 & & $0.00011)$ & 8 & & $0.00008)$ & 5 & \\
\hline
\end{tabular}

Spline Knots at LBW $<2,500$ grams and HBW $>4000$ grams

Variables included in the model: Model 1: All the outcomes were regressed on birth weight. Model 2: All the outcomes were regressed on birth weight variable and the child's BMI percentile in fifth grade. Model 3: All the outcomes were regressed on birth weight and the child's BMI percentile and additional covariates. Only covariates that were significant in the Spearman's correlation were used in the multiple regression analysis. Each non-significant covariate was deleted from the regression model one at a time. Variables retained in model for SBP: child's age, race (white vs. other), maternal health insurance status at time of delivery (non-Medicaid vs. Medicaid), and family history of cholesterol (yes vs. 
no); DBP: child's age, race (white vs. other), and family history of cholesterol (yes vs. no); TC: child's age, race (white vs. other), and family history of cholesterol (yes vs. no); LDL: child's age, gender, interaction between age*gender, and family history of cholesterol (yes vs. no); HDL: child's age, gender, race (white vs. other), family history of CVD (yes vs. no), maternal smoking status during pregnancy (yes vs. no), and maternal age at time of delivery; Non-HDL: child's age, family history of cholesterol (yes vs. no), and breastfeeding intention (breastfeed vs. both); TG: child's age, sex, race (white vs. other), family history of cholesterol (yes vs. no), family history of CVD (yes vs. no), and maternal education at birth.

CVD-Cardiovascular Disease; SBP-systolic blood pressure; DBP-diastolic blood pressure; TC-total cholesterol; LDL-low-density lipoprotein cholesterol; HDL-high-density lipoprotein; non-HDL-non-high-density lipoprotein cholesterol; cholesterol; TG-triglycerides 
Supplemental Table S2: Results of the Spline regression analysis for the association between birth weight and maternal CVD risk factors using merged data from the Birth Score Project (1994-2000) and the CARDIAC Project (2004-2010) for all infants who were born Full-Term $(\mathrm{N}=19,583)$

\begin{tabular}{|c|c|c|c|c|c|c|c|c|c|}
\hline \multirow[b]{2}{*}{$\begin{array}{l}\text { Mothers' } \\
\text { CVD risk } \\
\text { factors }\end{array}$} & \multirow[b]{2}{*}{$\begin{array}{l}\text { BTW } \\
\text { (grams) }\end{array}$} & \multicolumn{4}{|c|}{ Model 1} & \multicolumn{4}{|c|}{ Model 2} \\
\hline & & $\begin{array}{c}\text { Beta-coefficient } \\
\text { unstandardized } \\
(\mathbf{9 5 \%} \% \mathbf{C I}) \\
-0.04\end{array}$ & $\begin{array}{l}\text { Standardi } \\
\text { zed (Beta) }\end{array}$ & P-value & $\begin{array}{l}\text { Adjust } \\
\text { ed-R } R^{2}\end{array}$ & $\begin{array}{c}\text { Beta-coefficient } \\
\text { unstandardized } \\
(\mathbf{9 5 \%} \% \mathbf{C I}) \\
-0.031\end{array}$ & $\begin{array}{c}\text { Standard } \\
\text { ized } \\
\text { (Beta) }\end{array}$ & P-value & $\begin{array}{c}\text { Adjuste } \\
\text { d-R }^{2}\end{array}$ \\
\hline \multirow[t]{3}{*}{ TC } & $<2500$ & $\begin{array}{c}(-0.107-0.027) \\
0.046\end{array}$ & -0.537 & 0.239 & 0.0027 & $\begin{array}{c}(-0.098-0.035) \\
0.037\end{array}$ & -0.418 & 0.356 & 0.0238 \\
\hline & $2500-4000$ & $\begin{array}{c}(-0.022-0.115) \\
-0.005\end{array}$ & 0.608 & 0.186 & & $\begin{array}{c}(-0.031-0.105) \\
-0.004\end{array}$ & 0.483 & 0.289 & \\
\hline & $>4000$ & $\begin{array}{c}(-0.03-0.02) \\
-0.045\end{array}$ & -0.014 & 0.701 & & $\begin{array}{c}(-0.029-0.02) \\
-0.036\end{array}$ & -0.012 & 0.731 & \\
\hline \multirow[t]{3}{*}{ LDL } & $<2500$ & $\begin{array}{c}(-0.106-0.015) \\
0.049\end{array}$ & -0.689 & 0.141 & 0.002 & $\begin{array}{c}(-0.096-0.023) \\
0.04\end{array}$ & -0.554 & 0.234 & 0.0189 \\
\hline & $2500-4000$ & $\begin{array}{c}(-0.012-0.111) \\
0.001\end{array}$ & 0.738 & 0.118 & & $\begin{array}{c}(-0.021-0.101) \\
0.001\end{array}$ & 0.603 & 0.198 & \\
\hline & $>4000$ & $\begin{array}{c}(-0.021-0.023) \\
0.007\end{array}$ & 0.003 & 0.938 & & $\begin{array}{c}(-0.02-0.023) \\
0.001\end{array}$ & 0.005 & 0.901 & \\
\hline \multirow[t]{3}{*}{ HDL } & $<2500$ & $\begin{array}{c}(-0.019-0.033) \\
-0.006\end{array}$ & 0.24 & 0.601 & 0.007 & $\begin{array}{c}(-0.025-0.026) \\
-0.001\end{array}$ & 0.02 & 0.965 & 0.055 \\
\hline & $2500-4000$ & $\begin{array}{c}(-0.033-0.02) \\
-0.006\end{array}$ & -0.219 & 0.635 & & $\begin{array}{c}(-0.028-0.025) \\
-0.004\end{array}$ & -0.05 & 0.916 & \\
\hline & $>4000$ & $(-0.016-0.003)$ & -0.048 & 0.195 & & $(-0.013-0.006)$ & -0.028 & 0.458 & \\
\hline \multirow[t]{2}{*}{$\begin{array}{l}\text { Non- } \\
\text { HDL }\end{array}$} & $<2500$ & $\begin{array}{c}-0.047 \\
(-0.116-0.023) \\
0.052\end{array}$ & -0.599 & 0.191 & 0.0016 & $\begin{array}{c}-0.038 \\
(-0.109-0.033) \\
0.044\end{array}$ & -0.494 & 0.289 & 0.0221 \\
\hline & $2500-4000$ & $(-0.02-0.123)$ & 0.652 & 0.157 & & $(-0.029-0.116)$ & 0.551 & 0.24 & \\
\hline
\end{tabular}




\begin{tabular}{|c|c|c|c|c|c|c|c|c|c|}
\hline & $>4000$ & $\begin{array}{c}-0.001 \\
(-0.027-0.024) \\
-0.000104\end{array}$ & -0.004 & 0.91 & & $\begin{array}{c}-0.001 \\
(-0.026-0.024)\end{array}$ & -0.003 & 0.942 & \\
\hline \multirow[t]{6}{*}{ Log-TG } & $<2500$ & $\begin{array}{c}(-0.00115- \\
0.000941)\end{array}$ & -0.08978 & 0.8446 & 0.0012 & $\begin{array}{c}-0.000145 \\
(-0.00119-0.0009)\end{array}$ & -0.12458 & 0.7857 & 0.0033 \\
\hline & & 0.000151 & & & & 0.0002 & & & \\
\hline & & $(-0.000921-$ & & & & $(-0.000872-$ & & & \\
\hline & $2500-4000$ & $0.00122)$ & 0.12706 & 0.7829 & & $0.00127)$ & 0.16901 & 0.7142 & \\
\hline & & 0.000006 & & & & 0.000006 & & & \\
\hline & $>4000$ & $\begin{array}{c}(-0.000375- \\
0.000387)\end{array}$ & 0.00113 & 0.9755 & & $\begin{array}{c}(-0.000374- \\
0.000386)\end{array}$ & 0.00109 & 0.9763 & \\
\hline
\end{tabular}

Spline Knots at LBW $<2,500$ grams and HBW $>4000$ grams

Variables included in the model: Model 1: All the outcomes were regressed on birth weight. Model 2: All the outcomes were regressed on birth weight and additional covariates. Only covariates that were significant in the Spearman's correlation were used in the multiple regression analysis. Each non-significant covariate was deleted from the regression model one at a time. Variables retained in model for TC: mothers age when child in fifth grade, and breastfeeding intention (breastfeed vs. both); LDL: mothers age when child in fifth grade, breastfeeding intention (breastfeed vs. both), and smoking in the house (yes vs. no); HDL: mothers age when child in fifth grade, smoking in the house (yes vs. no), family history of CVD (yes vs. no), family history of diabetes (yes vs. no), number of previous pregnancies ( $\geq 1$ vs. 0); Non-HDL: mothers age when child in fifth grade, breastfeeding intention (breastfeed vs. both), and smoking in the house (yes vs. no); TG: mothers age when child in fifth grade and breastfeeding intention (breastfeed vs. both).

CVD-Cardiovascular Disease; SBP-systolic blood pressure; DBP-diastolic blood pressure; TC-total cholesterol; LDL-low-density lipoprotein cholesterol; HDL-high-density lipoprotein; non-HDL-non-high-density lipoprotein cholesterol; cholesterol; TG-triglycerides 


\section{Chapter 3}

Table 1: 2011 National Immunization Survey (NIS) and Pediatric Nutrition Surveillance System (PedNSS) breastfeeding rates nationally and in the state of WV. ${ }^{13,14}$

\begin{tabular}{lccccc}
\hline & \multicolumn{2}{c}{ NIS } & \multicolumn{3}{c}{ PedNSS } \\
& $\begin{array}{c}\text { National } \\
\mathbf{( \% )}\end{array}$ & $\begin{array}{l}\text { WV } \\
\mathbf{( \% )}\end{array}$ & $\begin{array}{c}\text { National } \\
\mathbf{( \% )}\end{array}$ & $\begin{array}{c}\text { WV } \\
\mathbf{( \% )}\end{array}$ & WV Rank \\
Ever breastfed & 79.2 & 59.3 & 66.3 & 44.7 & 41 \\
Breastfed at least 6 months & 49.4 & 29.3 & 26.0 & 13.5 & 38 \\
Breastfed at least 12 months & 26.7 & 15.9 & 17.9 & 10.2 & 30 \\
Exclusive breastfeeding 3 months & 40.7 & 28.3 & 10.8 & 2.9 & 27 \\
Exclusive breastfeeding 6 months & 18.8 & 12.2 & 6.3 & 0.3 & 28 \\
\hline
\end{tabular}


Table 2: Maternal and child characteristics at birth and in fifth grade using merged data from the Birth Score Project (1998-2003) and the CARDIAC Project (2010-2013) for all infants who were born Full-Term ( $\mathrm{N}=10457)$

\begin{tabular}{|c|c|c|c|}
\hline Variable & $\mathbf{N}$ & Missing & $\begin{array}{l}\text { Frequency } \\
(\%) / \text { Mean (SD) }\end{array}$ \\
\hline Sex & 10457 & & \\
\hline Female & & & $5740(54.89)$ \\
\hline Male & & & $4717(45.11)$ \\
\hline Race & 10070 & 387 & \\
\hline Others & & & $630(6.26)$ \\
\hline White & & & $9440(93.74)$ \\
\hline Marital Status (at birth) & 10457 & & \\
\hline Single & & & $2788(26.66)$ \\
\hline Married & & & $7669(73.34)$ \\
\hline $\begin{array}{l}\text { No. of previous pregnancy (at } \\
\text { birth) }\end{array}$ & 10457 & & \\
\hline 0 & & & $3651(34.91)$ \\
\hline$\geq 1$ & & & $6806(65.09)$ \\
\hline $\begin{array}{l}\text { Health Insurance of mother (at } \\
\text { birth) }\end{array}$ & 10457 & & \\
\hline Medicaid & & & $4841(46.29)$ \\
\hline Non-Medicaid & & & $5616(53.71)$ \\
\hline Family History of Cholesterol & 8879 & 1578 & \\
\hline No & & & $6119(68.92)$ \\
\hline Yes & & & $2760(31.08)$ \\
\hline Family History of CVD & 10457 & & \\
\hline No & & & $6114(58.47)$ \\
\hline Yes & & & $4343(41.53)$ \\
\hline Smoking during Pregnancy & 10395 & 62 & \\
\hline Yes & & & $2580(24.82)$ \\
\hline No & & & $7815(75.18)$ \\
\hline No. of Prenatal care visits & 10457 & & \\
\hline$<12$ & & & $4641(44.38)$ \\
\hline$\geq 12$ & & & $5816(55.62)$ \\
\hline Breastfeeding & 6833 & 3624 & \\
\hline No & & & $3883(56.83)$ \\
\hline Yes & & & $2950(43.17)$ \\
\hline Age of Child (years) & 10457 & 0 & $10.97(0.47)$ \\
\hline Maternal Age at Birth (years) & 10424 & 33 & $25.79(5.5)$ \\
\hline Maternal Education at Birth & 10405 & 52 & $12.87(2.12)$ \\
\hline Weight Gain during Pregnancy & 9597 & 860 & $30.79(14.66)$ \\
\hline
\end{tabular}




\begin{tabular}{llll}
\hline (lbs) & & & \\
Gestational Age (weeks) & 10457 & 0 & $39.07(1.12)$ \\
Birth Weight (grams) & 10452 & 5 & $3352.64(481.55)$ \\
BMI-Percentile & 10444 & 13 & $72.58(28)$ \\
Child SBP (mmHg) & 10254 & 203 & $108.43(11.75)$ \\
Child DBP (mmHg) & 10255 & 202 & $67.49(9.53)$ \\
Child TC (mg/dL) & 9740 & 717 & $157.7(27.51)$ \\
Child LDL (mg/dL) & 9725 & 732 & $89.99(24.41)$ \\
Child HDL (mg/dL) & 9740 & 717 & $50.14(12.37)$ \\
Child NON-HDL (mg/dL) & 9740 & 717 & $107.56(27.86)$ \\
Child TG (mg/dL) & 9739 & 718 & $92.28(54.99)$ \\
Child log TG & 9739 & 718 & $4.38(0.52)$ \\
Maternal TC (mg/dL) & 112 & 10345 & $182.67(33.62)$ \\
Maternal LDL (mg/dL) & 109 & 10348 & $110.64(27.87)$ \\
Maternal HDL (mg/dL) & 112 & 10345 & $51.55(12.73)$ \\
Maternal NON-HDL (mg/dL) & 112 & 10342 & $131.12(33.64)$ \\
Maternal TG (mg/dL) & 112 & 10342 & $106.47(73.95)$ \\
Maternal log TG & 112 & 10342 & $4.47(0.61)$ \\
CVD-Cardiovascular Disease; SBP-systolic blood pressure; DBP-diastolic blood pressure; TC- \\
total cholesterol; LDL-low-density lipoprotein cholesterol; HDL-high-density lipoprotein; non- \\
HDL-non-high-density lipoprotein cholesterol; cholesterol; TG-triglycerides \\
\hline
\end{tabular}


Table 3: Results of the Independent Sample T-test for the mean difference in blood pressure and lipid levels in both fifth grade children who were breastfed vs. not breastfed and mothers who breastfed vs. did not breastfed for children born Full-Term using merged data from the Birth Score Project (1998-2003) and the CARDIAC Project (2010-2013)

\begin{tabular}{|c|c|c|c|c|c|c|c|c|c|c|}
\hline & & \multicolumn{5}{|c|}{ Childhood CVD risk factors } & \multicolumn{4}{|c|}{ Maternal CVD risk factors } \\
\hline & & Breastfed & $\begin{array}{c}\text { Not } \\
\text { Breastfed }\end{array}$ & $\begin{array}{c}\text { Mean } \\
\text { Difference } \\
(95 \% \mathrm{CI})\end{array}$ & P-value & $\begin{array}{c}\text { Cohen's } \\
\text { d effect } \\
\text { size }\end{array}$ & $\begin{array}{c}\text { Breastf } \\
\text { ed }\end{array}$ & $\begin{array}{c}\text { Not } \\
\text { Breastfed }\end{array}$ & $\begin{array}{c}\text { Mean } \\
\text { Difference } \\
(95 \% \text { CI })\end{array}$ & $\begin{array}{c}\text { P- } \\
\text { value }\end{array}$ \\
\hline \multirow{2}{*}{$\begin{array}{l}\text { BMI } \\
\text { (Percentile) }\end{array}$} & $(\mathbf{N})$ & 2948 & 3877 & & & & & & & \\
\hline & Mean (SD) & $\begin{array}{l}70.2 \\
(28.6)\end{array}$ & $\begin{array}{c}74.2 \\
(27.5)\end{array}$ & $\begin{array}{c}-3.95^{*} \\
(-5.29,-2.62)\end{array}$ & $<0.0001$ & 0.14 & & & & \\
\hline \multirow[t]{2}{*}{ SBP } & $(\mathbf{N})$ & 2866 & 3781 & & & & & & & \\
\hline & $\begin{array}{l}\text { Mean (SD) } \\
\text { (N) }\end{array}$ & $\begin{array}{c}107.3 \\
(11.8) \\
2867\end{array}$ & $\begin{array}{c}108.6 \\
(11.9) \\
3781\end{array}$ & $\begin{array}{c}-1.3 * \\
(-1.97,-0.81)\end{array}$ & $<0.0001$ & 0.11 & & & & \\
\hline DBP & Mean (SD) & $\begin{array}{l}66.4 \\
(9.5)\end{array}$ & $\begin{array}{l}67.2 \\
(9.7)\end{array}$ & $\begin{array}{c}-0.8 * \\
(-1.26,-0.33)\end{array}$ & 0.0009 & 0.08 & & & & \\
\hline \multirow[t]{2}{*}{ TC } & $(\mathbf{N})$ & 2792 & 3644 & & & & 31 & 21 & & \\
\hline & $\begin{array}{l}\text { Mean (SD) } \\
\text { (N) }\end{array}$ & $\begin{array}{c}157.5 \\
(27.8) \\
2788\end{array}$ & $\begin{array}{c}157.8 \\
(27.9) \\
3639\end{array}$ & $\begin{array}{c}-0.3 \\
(-1.71,1.04)\end{array}$ & 0.6285 & & $\begin{array}{c}182 \\
(34.1) \\
31\end{array}$ & $\begin{array}{c}187 \\
(41.2) \\
20\end{array}$ & $\begin{array}{c}-4.9 \\
(-26,16.09)\end{array}$ & 0.6385 \\
\hline LDL & $\begin{array}{l}\text { Mean (SD) } \\
\text { (N) }\end{array}$ & $\begin{array}{c}89.4 \\
(24.6) \\
2792\end{array}$ & $\begin{array}{c}89.3 \\
(24.8) \\
3644\end{array}$ & $\begin{array}{c}0.1 \\
(-1.12,1.31)\end{array}$ & 0.8868 & & $\begin{array}{c}108.5 \\
(27.3) \\
31\end{array}$ & $\begin{array}{c}114.8 \\
(31.2) \\
21\end{array}$ & $\begin{array}{c}-6.3 \\
(-22.92,10.35)\end{array}$ & 0.4515 \\
\hline HDL & $\begin{array}{l}\text { Mean (SD) } \\
\text { (N) }\end{array}$ & $\begin{array}{c}51.0 \\
(12.4) \\
2792\end{array}$ & $\begin{array}{c}50.1 \\
(12.5) \\
3644\end{array}$ & $\begin{array}{c}0.9 * \\
(0.33,1.56)\end{array}$ & 0.0026 & 0.07 & $\begin{array}{c}52.7 \\
(11.1) \\
31\end{array}$ & $\begin{array}{c}50.4 \\
(12.7) \\
21\end{array}$ & $\begin{array}{c}2.3 \\
(-4.37,8.99)\end{array}$ & 0.49 \\
\hline Non-HDL & $\begin{array}{l}\text { Mean (SD) } \\
(\mathrm{N})\end{array}$ & $\begin{array}{c}106.4 \\
(27.9) \\
2791\end{array}$ & $\begin{array}{l}107.7 \\
(28.5) \\
3644\end{array}$ & $\begin{array}{c}-1.3 \\
(-2.70,0.11)\end{array}$ & 0.0705 & & $\begin{array}{c}129.3 \\
(36.0) \\
31\end{array}$ & $\begin{array}{c}136.5 \\
(36.3) \\
21\end{array}$ & $\begin{array}{c}-7.3 \\
(-27.77,13.24)\end{array}$ & 0.4799 \\
\hline
\end{tabular}




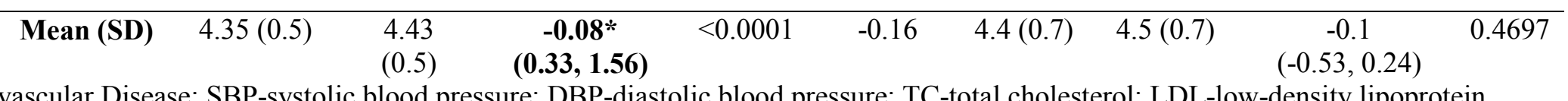

CVD-Cardiovascular Disease; SBP-systolic blood pressure; DBP-diastolic blood pressure; TC-total cholesterol; LDL-low-density lipoprotein cholesterol; HDL-high-density lipoprotein; non-HDL-non-high-density lipoprotein cholesterol; cholesterol; TG-triglycerides 
Table 4: Correlation between Breastfeeding and CVD risk factors for infants born Full-Term using merged data from the Birth Score Project (1998-2003) and the CARDIAC Project (20102013)

\begin{tabular}{rrrcc}
\hline $\begin{array}{l}\text { Childhood CVD risk } \\
\text { factors }\end{array}$ & $\mathbf{N}$ & $\begin{array}{c}\text { Correlation } \\
\text { Coefficient r }\end{array}$ & P-value \\
\hline BMI percentile & 6825 & $\mathbf{- 0 . 0 8}$ & $<\mathbf{0 . 0 0 0 1}$ \\
SBP & 6647 & $\mathbf{- 0 . 0 6}$ & $<\mathbf{0 . 0 0 0 1}$ \\
DBP & 6648 & $\mathbf{- 0 . 0 4}$ & $\mathbf{0 . 0 0 0 7}$ \\
TC & 6436 & -0.008 & 0.4941 \\
LDL & 6427 & 0.004 & 0.7731 \\
HDL & 6436 & $\mathbf{0 . 0 4}$ & $\mathbf{0 . 0 0 1 1}$ \\
Non-HDL & 6436 & -0.02 & 0.0632 \\
Log-TG & 6435 & $\mathbf{- 0 . 0 7}$ & $<\mathbf{0 . 0 0 0 1}$ \\
\hline Maternal CVD risk & & & 0.7406 \\
\hline & TC & 52 & -0.05 & 0.5743 \\
LDL & 51 & -0.08 & 0.3958 \\
HDL & 52 & 0.12 & 0.4554 \\
Non-HDL & 52 & -0.11 & 0.5935 \\
Log-TG & 52 & -0.08 & \\
\hline
\end{tabular}

CVD-Cardiovascular Disease; SBP-systolic blood pressure; DBP-diastolic blood pressure; TCtotal cholesterol; LDL-low-density lipoprotein cholesterol; HDL-high-density lipoprotein; nonHDL-non-high-density lipoprotein cholesterol; cholesterol; TG-triglycerides 
Table 5: Results of the multiple regression analysis for the association between reported history of breastfeeding and CVD risk factors of FifthGrade WV Children born Full-Term using merged data from the Birth Score Project (1998-2003) and the CARDIAC Project (2010-2013)

\begin{tabular}{|c|c|c|c|c|c|c|c|c|c|c|c|c|}
\hline \multirow{2}{*}{$\begin{array}{l}\text { Risk } \\
\text { factor } \\
\text { s } \\
\end{array}$} & \multicolumn{4}{|c|}{ Model 1} & \multicolumn{4}{|c|}{ Model 2} & \multicolumn{4}{|c|}{ Model 3} \\
\hline & $\begin{array}{c}\text { Unstandardiz } \\
\text { ed Regression } \\
\text { Coefficient } \\
(95 \% \text { CI })\end{array}$ & P-value & $\begin{array}{c}\text { Stand } \\
\text { ardize } \\
\text { d } \\
\text { Beta }\end{array}$ & $\mathbf{R}^{2}$ & $\begin{array}{c}\text { Unstandardiz } \\
\text { ed Regression } \\
\text { Coefficient } \\
(95 \% \text { CI })\end{array}$ & P-value & $\begin{array}{c}\text { Standar } \\
\text { dized } \\
\text { Beta }\end{array}$ & $\begin{array}{c}\text { Adjuste } \\
\text { d R }^{2}\end{array}$ & $\begin{array}{c}\text { Unstandardi } \\
\text { zed } \\
\text { Regression } \\
\text { Coefficient } \\
\text { (95\% CI) }\end{array}$ & $\begin{array}{c}P- \\
\text { value }\end{array}$ & $\begin{array}{l}\text { Stan } \\
\text { dard } \\
\text { ized } \\
\text { Beta }\end{array}$ & $\begin{array}{l}\text { Adjus } \\
\text { ted } R^{2}\end{array}$ \\
\hline SBP & $\begin{array}{c}-1.39 \\
(-1.97--0.81)\end{array}$ & $<0.0001$ & -0.06 & $\begin{array}{c}0.003 \\
3\end{array}$ & $\begin{array}{c}-0.77 \\
(-1.32--0.23)\end{array}$ & 0.0052 & -0.032 & 0.1188 & $\begin{array}{c}-0.43 \\
(-0.98-0.13)\end{array}$ & $\begin{array}{c}0.134 \\
9\end{array}$ & -0.02 & 0.1311 \\
\hline DBP & $\begin{array}{c}-0.79 \\
(-1.26--0.33)\end{array}$ & 0.0009 & -0.04 & $\begin{array}{c}0.001 \\
7\end{array}$ & $\begin{array}{c}-0.44 \\
(-0.89-0.02)\end{array}$ & 0.06 & -0.022 & 0.0633 & & & & \\
\hline TC & $\begin{array}{c}-0.34 \\
(-1.71-1.04)\end{array}$ & 0.6285 & -0.006 & 0.000 & $\begin{array}{c}-0.001 \\
(-1.37-1.37)\end{array}$ & 0.999 & $\stackrel{-}{0.00001}$ & 0.0069 & & & & \\
\hline LDL & $\begin{array}{c}0.09 \\
(-1.13-1.31)\end{array}$ & 0.8868 & 0.002 & 0.000 & $\begin{array}{c}0.62 \\
(-0.59-1.83)\end{array}$ & 0.3164 & 0.012 & 0.0231 & & & & \\
\hline HDL & $\begin{array}{c}0.95 \\
(0.33-1.56)\end{array}$ & 0.0026 & 0.04 & 0.001 & $\begin{array}{c}0.28 \\
(-0.29-0.86)\end{array}$ & 0.335 & 0.011 & 0.1378 & & & & \\
\hline $\begin{array}{l}\text { Non- } \\
\text { HDL }\end{array}$ & $\begin{array}{c}-1.29 \\
(-2.68-0.11)\end{array}$ & 0.0705 & -0.02 & $\begin{array}{c}0.000 \\
5\end{array}$ & $\begin{array}{c}-0.28 \\
(-1.64-1.07)\end{array}$ & 0.6824 & -0.005 & 0.0611 & & & & \\
\hline $\begin{array}{l}\text { Log } \\
\text { TG }\end{array}$ & $\begin{array}{c}-0.08 \\
(-0.1--0.05)\end{array}$ & $<0.0001$ & -0.07 & $\begin{array}{c}0.005 \\
3\end{array}$ & $\begin{array}{c}-0.05 \\
(-0.07--0.03)\end{array}$ & $<0.0001$ & -0.048 & 0.1302 & $\begin{array}{c}-0.04 \\
(-0.06-- \\
0.01)\end{array}$ & 0.008 & -0.03 & 0.1499 \\
\hline $\begin{array}{l}\text { Variabl } \\
\text { regress } \\
\text { the chil } \\
\text { regress } \\
\text { educati } \\
\text { number }\end{array}$ & $\begin{array}{l}\text { included in the } \\
\text { d on breastfed va } \\
\text { 's BMI percentile } \\
\text { on analysis. Cova } \\
\text { n), maternal heal } \\
\text { of previous pregn } \\
\text { cy in lbs. Covaria }\end{array}$ & $\begin{array}{l}\text { model: Mo } \\
\text { riable and t } \\
\text { and additi } \\
\text { riates inclu } \\
\text { h insuranc } \\
\text { ancies asse } \\
\text { tes that we }\end{array}$ & $\begin{array}{l}\text { del 1: Al } \\
\text { he child's } \\
\text { onal covi } \\
\text { ded child } \\
\text { e status a } \\
\text { ssed at b }\end{array}$ & $\begin{array}{l}\text { he ou } \\
\text { 3MI p } \\
\text { ates. } \\
\text { age, } \\
\text { ime o } \\
\text { h }(>=\end{array}$ & $\begin{array}{l}\text { omes were regre } \\
\text { rcentile in fifth g } \\
\text { nly covariates th } \\
\text { ender, race, infant } \\
\text { delivery (non-M } \\
\text { vs. 0), maternal } \\
\text { in the Spearman's }\end{array}$ & $\begin{array}{l}\text { sed on bre } \\
\text { de. Mode } \\
\text { were sig } \\
\text { birth weig } \\
\text { dicaid vs. } \\
\text { moking st }\end{array}$ & $\begin{array}{l}\text { tfed var } \\
\text { 3: All th } \\
\text { ficant in } \\
\text { in gram } \\
\text { ledicaid) } \\
\text { us durin }\end{array}$ & $\begin{array}{l}\text { le (yes vs } \\
\text { utcomes } \\
\text { Spearma } \\
\text { naternal } \\
\text { mily hist } \\
\text { regnancy }\end{array}$ & $\begin{array}{l}\text { 10). Model 2: } \\
\text { re regressed or } \\
\text { s correlation } \\
\text { ucation status } \\
\text { y of hyperchol } \\
\text { es vs. no), wei }\end{array}$ & $\begin{array}{l}1 \text { the } \\
\text { breast } \\
\text { re use } \\
\text { birth } \\
\text { sterole } \\
\text { ht gai }\end{array}$ & $\begin{array}{l}\text { comes } \\
d \text { variab } \\
\text { in the } n \\
-17 \text { yea } \\
\text { ia (yes }\end{array}$ & $\begin{array}{l}\text { ere } \\
\text { le and } \\
\text { ultiple } \\
\text { s of } \\
\text { s. no), } \\
\text { hild, }\end{array}$ \\
\hline
\end{tabular}


infant birth weight in grams, marital status of the mother at birth, number of prenatal care visits. Each non-significant covariate was deleted from the regression model one at a time. Variables retained in model for SBP: child's age, maternal education at birth. For TG: Child's age, sex, race, maternal education at birth, number of previous pregnancies, and family history of cholesterol.

CVD-Cardiovascular Disease; SBP-systolic blood pressure; DBP-diastolic blood pressure; TC-total cholesterol; LDL-low-density lipoprotein cholesterol; HDL-high-density lipoprotein; non-HDL-non-high-density lipoprotein cholesterol; cholesterol; TG-triglycerides 
Table 6: Results of the multiple regression analysis for all the variables in the model to predict Triglyceride of Fifth-Grade WV Children born Full Term using merged data from the Birth Score Project (1998-2003) and the CARDIAC Project (2010-2013)

\begin{tabular}{|c|c|c|c|c|c|c|c|c|c|c|}
\hline & \multicolumn{5}{|c|}{ Full Model } & \multicolumn{5}{|c|}{ Covariate Model } \\
\hline & $\begin{array}{c}\text { Unstandardized } \\
\text { regression } \\
\text { coefficients } \\
(95 \% \mathrm{CI})\end{array}$ & $\begin{array}{c}\text { Standa } \\
\text { rdized } \\
\text { Beta }\end{array}$ & $\begin{array}{c}\text { t } \\
\text { Value }\end{array}$ & P-value & $\begin{array}{c}\text { Adjusted } \\
\mathbf{R}^{\mathbf{2}}\end{array}$ & $\begin{array}{c}\text { Unstandardized } \\
\text { regression } \\
\text { coefficients } \\
(95 \% \mathrm{CI})\end{array}$ & $\begin{array}{c}\text { Stand } \\
\text { ardiz } \\
\text { ed } \\
\text { Beta }\end{array}$ & $\begin{array}{c}\text { t } \\
\text { Value }\end{array}$ & P-value & $\begin{array}{l}\text { Adjus } \\
\text { ted } R^{2}\end{array}$ \\
\hline Intercept & $\begin{array}{c}3.31 \\
(2.97-3.64)\end{array}$ & 0.00 & 19.3 & $<0.0001$ & 0.1499 & $\begin{array}{c}3.17 \\
(2.89-3.44)\end{array}$ & 0 & 22.56 & $<0.0001$ & 0.1486 \\
\hline Age (Years) & $\begin{array}{c}0.06 \\
(0.04-0.09)\end{array}$ & 0.06 & 4.4 & $<0.0001$ & & $\begin{array}{c}0.072 \\
(0.05-0.10)\end{array}$ & 0.06 & 6.08 & $<0.0001$ & \\
\hline $\begin{array}{l}\text { Gender } \\
\text { (Male vs. Female) }\end{array}$ & $\begin{array}{c}-0.11 \\
(-0.14--0.08)\end{array}$ & -0.10 & -8.24 & $<0.0001$ & & $\begin{array}{c}-0.11 \\
(-0.13--0.09)\end{array}$ & -0.11 & -10.48 & $<0.0001$ & \\
\hline $\begin{array}{l}\text { Race } \\
\text { (White vs. Others) }\end{array}$ & $\begin{array}{c}0.14 \\
(0.09-0.19)\end{array}$ & 0.07 & 5.3 & $<0.0001$ & & $\begin{array}{c}0.12 \\
(0.08-0.17)\end{array}$ & 0.06 & 5.58 & $<0.0001$ & \\
\hline $\begin{array}{l}\text { History of } \\
\text { hypercholesterolemia }\end{array}$ & $\begin{array}{c}0.07 \\
(0.04-0.10)\end{array}$ & 0.06 & 5.04 & $<0.0001$ & & $\begin{array}{c}0.07 \\
(0.05-0.10)\end{array}$ & 0.06 & 6.16 & $<0.0001$ & \\
\hline $\begin{array}{l}\text { Number of Previous } \\
\text { pregnancies }(\geq 1 \text { vs. } 0)\end{array}$ & $\begin{array}{c}-0.04 \\
(-0.07--0.02)\end{array}$ & -0.04 & -3.12 & 0.0018 & & $\begin{array}{c}-0.03 \\
(-0.06--0.01)\end{array}$ & -0.03 & -3.01 & 0.0026 & \\
\hline $\begin{array}{l}\text { Maternal education } \\
\text { (Years) }\end{array}$ & $\begin{array}{c}-0.010 \\
(-0.017--0.004)\end{array}$ & -0.04 & -3.16 & 0.0016 & & $\begin{array}{c}-0.008 \\
(-0.014--0.004)\end{array}$ & -0.04 & -3.43 & 0.0006 & \\
\hline BMI percentile & $\begin{array}{c}0.0065 \\
(0.006-0.007)\end{array}$ & 0.35 & 27.45 & $<0.0001$ & & $\begin{array}{c}0.0065 \\
(0.006-0.007)\end{array}$ & 0.35 & 33.72 & $<0.0001$ & \\
\hline $\begin{array}{l}\text { Breastfed } \\
\text { (Yes vs. No) }\end{array}$ & $\begin{array}{c}-0.04 \\
(-0.06--0.01)\end{array}$ & -0.03 & -2.65 & 0.008 & & & & & & \\
\hline \multicolumn{11}{|c|}{$\begin{array}{l}\text { Only covariates that were significant in the Spearman's correlation were used in the multiple regression analysis. Covariates included child's age, } \\
\text { gender, race, infant birth weight in grams, maternal education status at birth (1-17 years of education), maternal health insurance status at time of } \\
\text { delivery (non-Medicaid vs. Medicaid), family history of hypercholesterolemia (yes vs. no), number of previous pregnancies assessed at birth ( } \geq 1 \\
\text { vs. 0), maternal smoking status during pregnancy (yes vs. no), weight gain during pregnancy in lbs. Covariates that were not significant in the } \\
\text { Spearman's correlation and were excluded: maternal age at birth of the index child, infant birth weight in grams, marital status of the mother at } \\
\text { birth, number of prenatal care visits. Each non-significant covariate was deleted from the regression model one at a time. These included infant }\end{array}$} \\
\hline
\end{tabular}


birth weight in grams, family history of hypercholesterolemia (yes vs. no), number of previous pregnancies assessed at birth ( $\geq 1$ vs. 0), maternal smoking status during pregnancy (yes vs. no), weight gain during pregnancy in lbs. WV, West Virginia; CI, confidence interval.

CVD-Cardiovascular Disease; SBP-systolic blood pressure; DBP-diastolic blood pressure; TC-total cholesterol; LDL-low-density lipoprotein cholesterol; HDL-high-density lipoprotein; non-HDL-non-high-density lipoprotein cholesterol; cholesterol; TG-triglycerides 


\section{Supplemental Table Chapter 3}

Supplemental Table S1: Maternal and Child Characteristics at Birth and in Fifth Grade using merged data from the Birth Score Project (1998-2003) and the CARDIAC Project (2010-2013) for All infants $(\mathrm{N}=11980)$

\begin{tabular}{|c|c|c|c|}
\hline Variable & $\mathbf{N}$ & Missing & $\begin{array}{l}\text { Frequency } \\
(\%) / \text { Mean (SD) }\end{array}$ \\
\hline Sex & 11980 & & \\
\hline Female & & & $6553(54.7)$ \\
\hline Male & & & $5427(45.3)$ \\
\hline Race & 11531 & 449 & \\
\hline Others & & & $729(6.32)$ \\
\hline White & & & $10802(93.68)$ \\
\hline Marital Status (at birth) & 11980 & & \\
\hline Single & & & $3181(26.55)$ \\
\hline Married & & & $8799(73.45)$ \\
\hline No. of previous pregnancy (at birth) & 11981 & & \\
\hline 0 & & & $4193(35)$ \\
\hline$\geq 1$ & & & $7787(65)$ \\
\hline Health Insurance of mother (at birth) & 11982 & & \\
\hline Medicaid & & & $5619(46.9)$ \\
\hline Non-Medicaid & & & $6361(53.1)$ \\
\hline Family History of Cholesterol & 10166 & 1814 & \\
\hline No & & & $6940(68.27)$ \\
\hline Yes & & & $3226(31.73)$ \\
\hline Family History of CVD & 11980 & & \\
\hline No & & & $6978(58.25)$ \\
\hline Yes & & & $5002(41.75)$ \\
\hline Smoking during Pregnancy & 11901 & 79 & \\
\hline Yes & & & $2960(24.87)$ \\
\hline No & & & $8941(75.13)$ \\
\hline No. of Prenatal care visits & 11980 & & \\
\hline$<12$ & & & $5486(45.79)$ \\
\hline$\geq 12$ & & & $6494(54.21)$ \\
\hline Breastfeeding & 7861 & 4119 & \\
\hline No & & & $4478(56.96)$ \\
\hline Yes & & & $3383(43.04)$ \\
\hline Age of Child (years) & 11980 & 0 & $10.97(0.47)$ \\
\hline Maternal Age at Birth (years) & 11945 & 35 & $25.84(5.54)$ \\
\hline Maternal Education at Birth & 11919 & 61 & $12.87(2.12)$ \\
\hline Weight Gain during Pregnancy (lbs) & 10907 & 1073 & $30.58(14.67)$ \\
\hline Gestational Age (weeks) & 11647 & 333 & $38.59(1.91)$ \\
\hline
\end{tabular}




\begin{tabular}{llll}
\hline Birth Weight (grams) & 11975 & 5 & $3259.34(569.19)$ \\
BMI-Percentile & 11967 & 13 & $72.31(28.19)$ \\
Child SBP (mmHg) & 11741 & 239 & $108.44(11.85)$ \\
Child DBP (mmHg) & 11742 & 238 & $67.46(9.52)$ \\
Child TC (mg/dL) & 11133 & 847 & $157.87(27.65)$ \\
Child LDL (mg/dL) & 11114 & 866 & $90.06(24.54)$ \\
Child HDL (mg/dL) & 11133 & 847 & $50.18(12.42)$ \\
Child NON-HDL (mg/dL) & 11133 & 847 & $107.69(28.1)$ \\
Child TG (mg/dL) & 11132 & 848 & $92.54(55.46)$ \\
Child log TG & 11132 & 848 & $4.38(0.53)$ \\
Maternal TC (mg/dL) & 127 & 11853 & $181.1(32.81)$ \\
Maternal LDL (mg/dL) & 123 & 11857 & $109.0(27.59)$ \\
Maternal HDL (mg/dL) & 127 & 11853 & $51.28(13.13)$ \\
Maternal NON-HDL (mg/dL) & 127 & 11853 & $129.77(33.24)$ \\
Maternal TG (mg/dL) & 127 & 11853 & $106.0(74.54)$ \\
Maternal log TG & 127 & 11853 & $4.47(0.62)$ \\
\hline
\end{tabular}

CVD-Cardiovascular Disease; SBP-systolic blood pressure; DBP-diastolic blood pressure; TCtotal cholesterol; LDL-low-density lipoprotein cholesterol; HDL-high-density lipoprotein; nonHDL-non-high-density lipoprotein cholesterol; cholesterol; TG-triglycerides 
Supplemental Table S2: Results of the Independent Sample T-test for the mean difference in blood pressure and lipid levels in both fifth grade children who were breastfed vs. not breastfed and mothers who breastfed vs. did not breastfed for all children (both pre-term and full-term) using merged data from the Birth Score Project (1998-2003) and the CARDIAC Project (2010-2013)

\begin{tabular}{|c|c|c|c|c|c|c|c|c|c|}
\hline & & \multicolumn{4}{|c|}{ Childhood CVD risk factors } & \multicolumn{4}{|c|}{ Maternal CVD risk factors } \\
\hline & & Breastfed & $\begin{array}{c}\text { Not } \\
\text { Breastfed }\end{array}$ & $\begin{array}{c}\text { Mean } \\
\text { Difference } \\
(95 \% \text { CI })\end{array}$ & P-value & Breastfed & $\begin{array}{c}\text { Not } \\
\text { Breastfed }\end{array}$ & $\begin{array}{c}\text { Mean } \\
\text { Difference } \\
(95 \% \text { CI })\end{array}$ & P-value \\
\hline \multirow[t]{2}{*}{ SBP } & $(\mathrm{N})$ & 3286 & 4357 & & & & & & \\
\hline & Mean (SD) & $\begin{array}{l}107.4 \\
(12.0)\end{array}$ & $\begin{array}{l}108.7 \\
(11.9)\end{array}$ & $\begin{array}{c}-1.3 \\
(-1.8--0.8)\end{array}$ & $<0.0001$ & & & & \\
\hline \multirow[t]{2}{*}{ DBP } & $(\mathrm{N})$ & 3287 & 4357 & & & & & & \\
\hline & Mean (SD) & $\begin{array}{l}66.4 \\
(9.6)\end{array}$ & $\begin{array}{l}67.2 \\
(9.6)\end{array}$ & $\begin{array}{c}-0.8 \\
(-1.2--0.3)\end{array}$ & 0.0005 & & & & \\
\hline \multirow[t]{2}{*}{ TC } & $(\mathrm{N})$ & 3199 & 4194 & & & 34 & 25 & & \\
\hline & Mean (SD) & $\begin{array}{l}157.6 \\
(27.8)\end{array}$ & $\begin{array}{l}157.9 \\
(27.8)\end{array}$ & $\begin{array}{c}-0.3 \\
(-1.6-0.6)\end{array}$ & 0.5845 & $\begin{array}{l}180.0 \\
(33.1)\end{array}$ & $\begin{array}{l}186.0 \\
(39.3)\end{array}$ & $\begin{array}{c}-6.0 \\
(-25.0-12.0)\end{array}$ & 0.527 \\
\hline \multirow[t]{2}{*}{ LDL } & $(\mathrm{N})$ & 3193 & 4188 & & & 34 & 24 & & \\
\hline & $\begin{array}{l}\text { Mean (SD) } \\
\text { (N) }\end{array}$ & $\begin{array}{c}89.3 \\
(24.6) \\
3199\end{array}$ & $\begin{array}{c}89.4 \\
(24.7) \\
4194\end{array}$ & $\begin{array}{c}-0.1 \\
\left(-1.2^{-1.0)}\right.\end{array}$ & 0.864 & $\begin{array}{c}106.5 \\
(27.0) \\
34\end{array}$ & $\begin{array}{c}113.0 \\
(30.7) \\
25\end{array}$ & $\begin{array}{c}-6.5 \\
(-21.8-8.7)\end{array}$ & 0.3961 \\
\hline HDL & $\begin{array}{l}\text { Mean (SD) } \\
\text { (N) }\end{array}$ & $\begin{array}{c}51.0 \\
(12.3) \\
3199\end{array}$ & $\begin{array}{c}50.2 \\
(12.7) \\
4194\end{array}$ & $\begin{array}{c}0.8 \\
(0.2-1.4)\end{array}$ & 0.0066 & $\begin{array}{c}52.5 \\
(11.8) \\
34\end{array}$ & $\begin{array}{c}51.1 \\
(12.4) \\
25\end{array}$ & $\begin{array}{c}1.4 \\
(-4.9-7.7)\end{array}$ & 0.6593 \\
\hline Non-HDL & $\begin{array}{l}\text { Mean (SD) } \\
\text { (N) }\end{array}$ & $\begin{array}{c}106.5 \\
(28.1) \\
3198\end{array}$ & $\begin{array}{l}107.7 \\
(28.4) \\
4194\end{array}$ & $\begin{array}{c}-1.2 \\
(-2.5-0.1)\end{array}$ & 0.0812 & $\begin{array}{c}127.5 \\
(35.3) \\
36\end{array}$ & $\begin{array}{c}134.9 \\
(36.1) \\
25\end{array}$ & $\begin{array}{c}-7.4 \\
(-26.2-11.4)\end{array}$ & 0.4327 \\
\hline Log-TG & Mean (SD) & $\begin{array}{l}4.36 \\
(0.5)\end{array}$ & $\begin{array}{l}4.42 \\
(0.5)\end{array}$ & $\begin{array}{c}-0.06 \\
(-0.08--0.04)\end{array}$ & $<0.0001$ & $\begin{array}{c}4.4 \\
(0.7)\end{array}$ & $\begin{array}{c}4.5 \\
(0.7)\end{array}$ & $\begin{array}{c}-0.12 \\
(-0.49-0.25)\end{array}$ & 0.5152 \\
\hline
\end{tabular}


Supplemental Table 3S: Correlation between breastfeeding and maternal and childhood CVD risk factors for all infants using merged data from the Birth Score Project (1998-2003) and the CARDIAC Project (2010-2013) as well examining the association for maternal variables using imputed dataset.

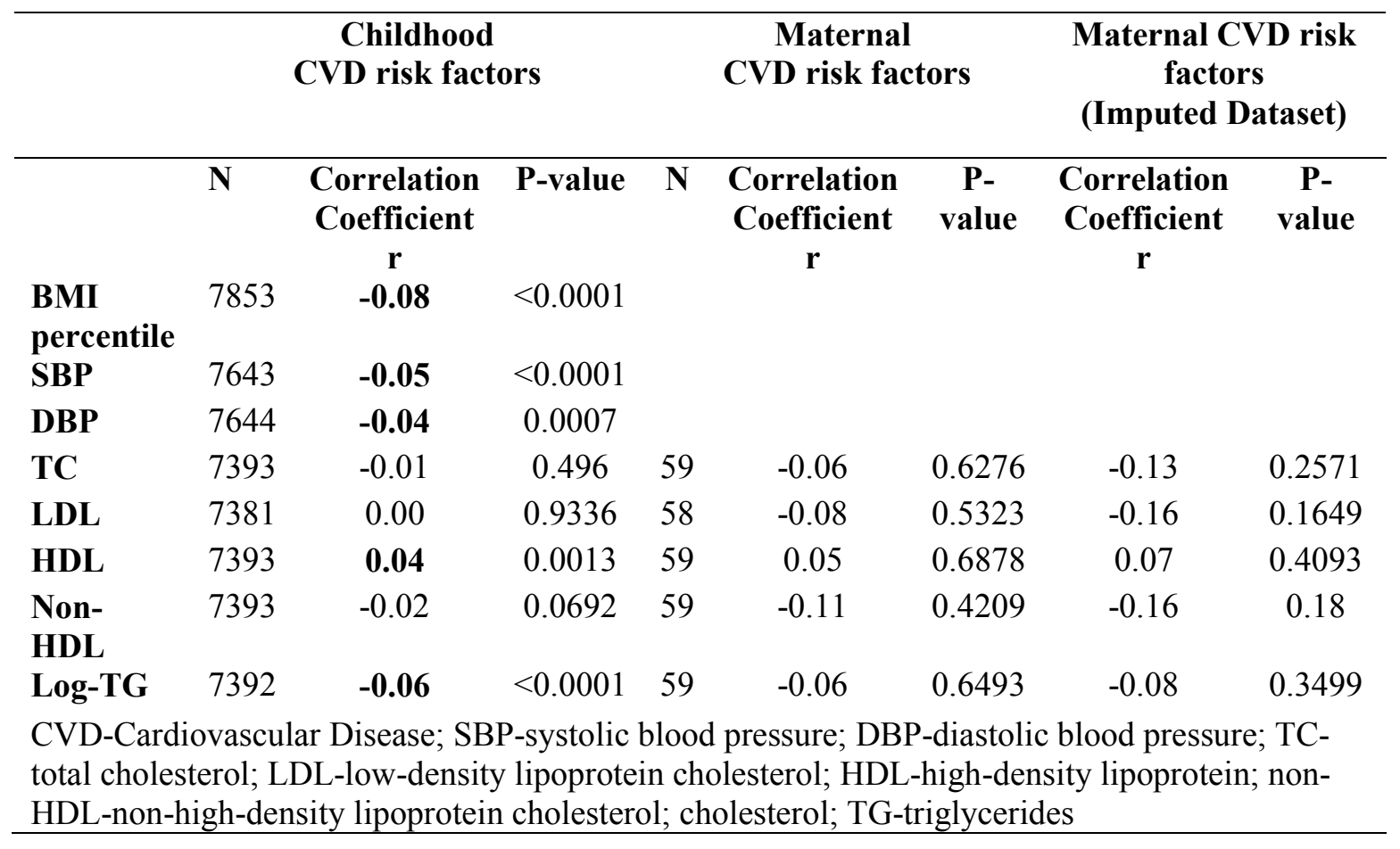


Supplemental Table S4: Results of the multiple regression analysis for the association between reported history of breastfeeding and CVD risk factors of Fifth-Grade WV Children for All infants (Preterm and Full term) using merged data from the Birth Score Project (1998-2003) and the CARDIAC Project (2010-2013)

\begin{tabular}{|c|c|c|c|c|c|c|c|c|c|c|c|c|}
\hline \multirow{2}{*}{$\begin{array}{l}\text { CVD } \\
\text { risk } \\
\text { factors }\end{array}$} & \multicolumn{4}{|c|}{ Model 1} & \multicolumn{4}{|c|}{ Model 2} & \multicolumn{4}{|c|}{ Model 3} \\
\hline & $\begin{array}{c}\text { Unstandar } \\
\text { dized } \\
\text { Regression } \\
\text { Coefficient }\end{array}$ & $\begin{array}{c}P- \\
\text { value }\end{array}$ & $\begin{array}{c}\text { Standar } \\
\text { dized } \\
\text { Beta }\end{array}$ & $\mathbf{R}^{2}$ & $\begin{array}{c}\text { Unstandar } \\
\text { dized } \\
\text { Regression } \\
\text { Coefficient }\end{array}$ & $\begin{array}{c}\text { P- } \\
\text { value }\end{array}$ & $\begin{array}{c}\text { Standar } \\
\text { dized } \\
\text { Beta }\end{array}$ & $\begin{array}{c}\text { Adjusted } \\
\mathbf{R}^{2}\end{array}$ & $\begin{array}{c}\text { Unstandar } \\
\text { dized } \\
\text { Regression } \\
\text { Coefficient }\end{array}$ & $\begin{array}{c}P- \\
\text { value }\end{array}$ & $\begin{array}{c}\text { Stan } \\
\text { dardi } \\
\text { zed } \\
\text { Beta }\end{array}$ & $\begin{array}{c}\text { Adjust } \\
\text { ed } R^{2}\end{array}$ \\
\hline SBP & -1.39 & 0.0001 & -0.054 & 0.003 & -0.73 & 0.0046 & -0.031 & 0.1195 & -0.34 & 0.2042 & -0.01 & 0.132 \\
\hline DBP & -0.75 & 0.0007 & -0.039 & 0.0015 & -0.41 & 0.0557 & -0.021 & 0.0669 & & & & \\
\hline TC & -0.36 & 0.5845 & -0.006 & 0.000 & -0.03 & 0.9666 & 0.000 & 0.0078 & & & & \\
\hline LDL & -0.10 & 0.864 & 0.002 & 0.000 & 0.42 & 0.4663 & 0.008 & 0.0256 & & & & \\
\hline HDL & 0.80 & 0.0066 & 0.032 & 0.001 & 0.17 & 0.5402 & 0.007 & 0.1438 & & & & \\
\hline $\begin{array}{l}\text { Non- } \\
\text { HDL }\end{array}$ & -1.16 & 0.0812 & -0.020 & 0.0004 & -0.28 & 0.762 & -0.003 & 0.0657 & & & & \\
\hline Log-TG & -0.06 & 0.0001 & -0.059 & 0.0034 & -0.04 & 0.0015 & -0.035 & 0.1328 & -0.03 & 0.046 & -0.02 & 0.1527 \\
\hline $\begin{array}{l}\text { Variables } \\
\text { breastfed } \\
\text { BMI perc } \\
\text { analysis. } \\
\text { maternal } \\
\text { previous } \\
\text { Covariate } \\
\text { in grams, } \\
\text { one at a ti } \\
\text { birth, nun } \\
\text { CVD-Car } \\
\text { cholester }\end{array}$ & $\begin{array}{l}\text { included in th } \\
\text { variable and th } \\
\text { entile and addi } \\
\text { Covariates incl } \\
\text { lealth insuranc } \\
\text { regnancies ass } \\
\text { that were not } \\
\text { marital status } \\
\text { me. Variables } \\
\text { ber of previou } \\
\text { diovascular Di } \\
\text { 1; HDL-high-d }\end{array}$ & $\begin{array}{l}\text { model: I } \\
\text { e child's I } \\
\text { ional cov } \\
\text { uded chil } \\
\text { e status at } \\
\text { essed at b } \\
\text { significat } \\
\text { f the mot } \\
\text { etained it } \\
\text { s pregnan } \\
\text { sease; SB } \\
\text { ensity lip }\end{array}$ & $\begin{array}{l}\text { del 1: All } \\
\text { II percent } \\
\text { ates. Onl } \\
\text { age, gend } \\
\text { me of del } \\
\text { h ( }>=1 \text { v } \\
\text { n the Spe } \\
\text { at birth, } \\
\text { odel for } \\
\text { s, and fa } \\
\text { ystolic b }\end{array}$ & $\begin{array}{l}\text { e outcor } \\
\text { in fifth } \\
\text { covariate } \\
\text {, race, in } \\
\text { ery (non- } \\
\text { 0), mater } \\
\text { man's co } \\
\text { umber of } \\
\text { 3P: child } \\
\text { ly histor } \\
\text { od pressu } \\
\text { HDL-no }\end{array}$ & $\begin{array}{l}\text { s were regre } \\
\text { ade. Model } \\
\text { that were sig } \\
\text { nt birth weig } \\
\text { ledicaid vs. I } \\
\text { l smoking st } \\
\text { elation and } \\
\text { renatal care } \\
\text { age, materna } \\
\text { of cholestero } \\
\text {; DBP-diast } \\
\text { high-density }\end{array}$ & $\begin{array}{l}\text { ed on b } \\
\text { All the } \\
\text { ificant it } \\
\text { t in grat } \\
\text { edicaid) } \\
\text { us durir } \\
\text { ere excl } \\
\text { sits. Eac } \\
\text { educatic } \\
\text { Additio } \\
\text { ic blooc } \\
\text { ipoprote }\end{array}$ & $\begin{array}{l}\text { stfed var } \\
\text { tcomes w } \\
\text { ne Spearn } \\
\text { materna } \\
\text { amily his } \\
\text { pregnanc } \\
\text { ed: mater } \\
\text { non-signi } \\
\text { at birth. I } \\
\text { lly gestat } \\
\text { cessure; T } \\
\text { cholester }\end{array}$ & $\begin{array}{l}\text { ole. Model } \\
\text { e regresse } \\
\text { n's correla } \\
\text { ducation s } \\
\text { y of hype } \\
\text { yes vs. no } \\
\text { l age at bi } \\
\text { ant covari } \\
\text { TG: Chil } \\
\text { hal age wa } \\
\text {-total chol } \\
\text {; cholester }\end{array}$ & $\begin{array}{l}\text { All the out } \\
\text { on breastfed } \\
\text { on were use } \\
\text { tus at birth ( } \\
\text { holesterolem } \\
\text { weight gain } \\
\text { h of the inde } \\
\text { te was delete } \\
\text { s age, sex, ra } \\
\text { adjusted for } \\
\text { terol; LDL-1 } \\
\text {; TG-triglyc }\end{array}$ & $\begin{array}{l}\text { omes w } \\
\text { ariable } \\
\text { in the } \mathrm{n} \\
-17 \text { yea } \\
\text { a (yes v } \\
\text { uring } \mathrm{p} \\
\text { child, } \mathrm{i} \\
\text { from tl } \\
\mathrm{e} \text {, mate } \\
\text { ut was } \\
\mathrm{W} \text {-dens } \\
\text { cides }\end{array}$ & $\begin{array}{l}\text { e regre } \\
\text { dd the } \\
\text { ltiple } r \\
\text { of edu } \\
\text { no), nu } \\
\text { gnancy } \\
\text { ant bir } \\
\text { regres } \\
\text { al educ } \\
\text { t signi } \\
\text { lipop }\end{array}$ & $\begin{array}{l}\text { d on } \\
\text { ld's } \\
\text { ession } \\
\text { tion), } \\
\text { oer of } \\
\text { lbs. } \\
\text { weight } \\
\text { n model } \\
\text { on at } \\
\text { int. } \\
\text { in }\end{array}$ \\
\hline
\end{tabular}


Supplemental Table S5: Results of the multiple regression analysis for all the variables in the model to predict Triglyceride of Fifth-Grade WV Children for All infants (Preterm and Full term) using merged data from the Birth Score Project (1998-2003) and the CARDIAC Project (20102013)

\begin{tabular}{|c|c|c|c|c|c|c|c|c|c|c|}
\hline & \multicolumn{5}{|c|}{ Full Model } & \multicolumn{5}{|c|}{ Covariate Model } \\
\hline & $\begin{array}{l}\text { Unstandardize } \\
\text { d regression } \\
\text { coefficients } \\
(95 \% \mathrm{CI})\end{array}$ & $\begin{array}{l}\text { Standa } \\
\text { rdized } \\
\text { Beta }\end{array}$ & t Value & P-value & $\begin{array}{l}\text { Adjust } \\
\text { ed } \mathbf{R}^{2}\end{array}$ & $\begin{array}{l}\text { Unstandardize } \\
\text { d regression } \\
\text { coefficients } \\
(95 \% \mathrm{CI})\end{array}$ & $\begin{array}{l}\text { Standar } \\
\text { dized } \\
\text { Beta }\end{array}$ & $\begin{array}{c}\mathrm{t} \\
\text { Value }\end{array}$ & P-value & $\begin{array}{c}\text { Adjust } \\
\text { ed } R^{2}\end{array}$ \\
\hline Intercept & $\begin{array}{c}3.33 \\
(3.02,3.65)\end{array}$ & 0.00 & 20.82 & $<0.0001$ & 0.1527 & $\begin{array}{c}3.184 \\
(2.928,3.44)\end{array}$ & 0.00 & 24.38 & $<0.0001$ & 0.1518 \\
\hline Age & $\begin{array}{c}0.06 \\
(0.03,0.08)\end{array}$ & 0.05 & 4.28 & $<0.0001$ & & $\begin{array}{c}0.068 \\
(0.046,0.089)\end{array}$ & 0.06 & 6.16 & $<0.0001$ & \\
\hline $\begin{array}{l}\text { Gender } \\
\text { (Male vs. Female) }\end{array}$ & $\begin{array}{c}-0.11 \\
(-0.13,-0.08)\end{array}$ & -0.10 & -8.50 & $<0.0001$ & & $\begin{array}{c}-0.113 \\
(-0.133,-0.093)\end{array}$ & -0.11 & -11.06 & $<0.0001$ & \\
\hline $\begin{array}{l}\text { Race } \\
\text { (White vs. Others) }\end{array}$ & $\begin{array}{c}0.14 \\
(0.09,0.19)\end{array}$ & 0.07 & 5.72 & $<0.0001$ & & $\begin{array}{c}0.135 \\
(0.094,0.176)\end{array}$ & 0.06 & 6.46 & $<0.0001$ & \\
\hline $\begin{array}{l}\text { History of } \\
\text { hypercholesterolemia } \\
\text { (Yes vs. No) }\end{array}$ & $\begin{array}{c}0.08 \\
(0.06,0.11)\end{array}$ & 0.07 & 6.07 & $<0.0001$ & & $\begin{array}{c}0.08 \\
(0.058,0.101)\end{array}$ & 0.07 & 7.29 & $<0.0001$ & \\
\hline $\begin{array}{l}\text { Number of Previous } \\
\text { pregnancies ( }>=1 \text { vs. 0) }\end{array}$ & $\begin{array}{c}-0.04 \\
(-0.06,-0.01)\end{array}$ & -0.03 & -2.79 & 0.0053 & & $\begin{array}{c}-0.031 \\
(-0.052,-0.011)\end{array}$ & -0.03 & -2.94 & 0.0032 & \\
\hline $\begin{array}{l}\text { Maternal education } \\
\text { (Years) }\end{array}$ & $\begin{array}{c}-0.01 \\
(-0.01,-0.003)\end{array}$ & -0.04 & -2.94 & 0.0033 & & $\begin{array}{c}-0.008 \\
(-0.013,-0.003)\end{array}$ & -0.03 & -3.24 & 0.0012 & \\
\hline BMI percentile & $\begin{array}{c}0.01 \\
(0.01,0.01)\end{array}$ & 0.36 & 30.22 & $<0.0001$ & & $\begin{array}{c}0.007 \\
(0.006,0.007)\end{array}$ & 0.35 & 36.60 & $<0.0001$ & \\
\hline $\begin{array}{l}\text { Breastfed } \\
\text { (Yes vs. No) }\end{array}$ & $\begin{array}{c}-0.03 \\
(-0.05,-0.0005)\end{array}$ & -0.02 & -2.00 & 0.0460 & & & & & & \\
\hline
\end{tabular}


Only covariates that were significant in the Spearman's correlation were used in the multiple regression analysis. Covariates included child's age, gender, race, infant birth weight in grams, maternal education status at birth (1-17 years of education), maternal health insurance status at time of delivery (non-Medicaid vs. Medicaid), family history of hypercholesterolemia (yes vs. no), number of previous pregnancies assessed at birth ( $>=1$ vs. 0), maternal smoking status during pregnancy (yes vs. no), weight gain during pregnancy in lbs. Covariates that were not significant in the Spearman's correlation and were excluded: maternal age at birth of the index child, infant birth weight in grams, marital status of the mother at birth, No. of prenatal care visits. Each non-significant covariate was deleted from the regression model one at a time. These included infant birth weight in grams, family history of hypercholesterolemia (yes vs. no), number of previous pregnancies assessed at birth ( $>=1 \mathrm{vs.} 0$ ), maternal smoking status during pregnancy (yes vs. no), weight gain during pregnancy in lbs. WV, West Virginia; CI, confidence interval. 


\section{Chapter 4}

Table 1: Previous SRs and MA examining the association between childhood obesity and selected adult CVD risk factors

\begin{tabular}{|c|c|c|c|}
\hline $\begin{array}{c}\text { Authors, year study } \\
\text { published }\end{array}$ & $\begin{array}{l}\text { Type of } \\
\text { study } \\
\text { design }\end{array}$ & $\begin{array}{l}\text { No. of studies included } \\
\text { and outcome of interest }\end{array}$ & Conclusion \\
\hline Park et al., $2012^{38}$ & SR & 5 studies for $\mathrm{HT}^{107,128-131}$ & $\begin{array}{l}\text { Increase in BMI/overweight in childhood was associated with increased risk of } \\
\text { HT. } 2 \text { out of } 5 \text { studies that adjusted for adult BMI found no association. The } \\
\text { review was unable to confirm the presence of long-term health effects of } \\
\text { childhood obesity independent of its effects on adult BMI. This conclusion was } \\
\text { not made for HT exclusively but the authors made a general statement. }\end{array}$ \\
\hline Lloyd et al., $2012^{18}$ & SR & $\begin{array}{l}5 \text { studies for lipids (TC, } \\
\text { TG, LDL, and } \\
\text { HDL) })^{4,116,132-134}\end{array}$ & $\begin{array}{l}\text { Little evidence that childhood obesity is an independent risk factor for adult } \\
\text { blood lipid status. }\end{array}$ \\
\hline Lloyd et al., $2010^{37}$ & SR & $\begin{array}{l}8 \text { studies for blood } \\
\text { pressure (SBP and } \\
\text { DBP })^{4,107,116,131,133,135-137}\end{array}$ & $\begin{array}{l}\text { Little evidence that childhood obesity is an independent risk factor for CVD } \\
\text { risk (SBP and DBP). The relationships observed are dependent on the tracking } \\
\text { of BMI from childhood to adulthood. }\end{array}$ \\
\hline Reilly et al., $2011^{39}$ & SR & $\begin{array}{l}4 \text { studies for } \\
\mathrm{HT}^{107,131,138,139}\end{array}$ & $\begin{array}{l}\text { Significant increase in adult HT with overweight and obesity during childhood } \\
\text { and adolescence. The SR did not mention if the studies included controlled for } \\
\text { adult weight status. }\end{array}$ \\
\hline Juonala et al., $2011^{40}$ & MA & $\begin{array}{l}\text { Studies from 4-cohorts- } \\
\text { BHS, MS, CDAH, and } \\
\text { YFS }\end{array}$ & $\begin{array}{l}\text { Childhood obesity was significantly associated with } \mathrm{HT} \text { even after adjustment } \\
\text { for adult obesity (relative risk, } 1.5 ; 95 \% \text { CI: } 1.1,2.1 ; \mathrm{P}=0.009 \text { ). For } \\
\text { dyslipidemias, the effect of childhood adiposity was reduced and became non- } \\
\text { significant when adult obesity was taken into account. }\end{array}$ \\
\hline
\end{tabular}


Table 2: Item-by-item results using the AMSTAR assessment instrument for previous SRs and MA

\begin{tabular}{|c|c|c|c|c|c|c|c|c|c|c|c|c|c|c|}
\hline Authors & Year & 1 & 2 & 3 & 4 & $5 *$ & 6 & 7 & 8 & 9 & 10 & 11 & 12 & $\begin{array}{c}\text { Total score Row } \\
\text { (\%) }\end{array}$ \\
\hline Park et al. ${ }^{38}$ & 2012 & SR & Yes & Yes & Yes & No & Yes & Yes & Yes & No & NA & Yes & Yes & $8 / 10=80 \%$ \\
\hline Lloyd et al. ${ }^{18}$ & 2012 & SR & Yes & Yes & Yes & No & No & Yes & Yes & Yes & NA & No & Yes & $7 / 10=70 \%$ \\
\hline Lloyd et al. ${ }^{37}$ & 2010 & SR & Yes & Yes & Yes & No & No & Yes & Yes & Yes & NA & No & No & $6 / 10=60 \%$ \\
\hline Reilly et al. ${ }^{39}$ & 2011 & SR & Yes & No & Yes & No & No & Yes & No & No & NA & No & Yes & $4 / 10=40 \%$ \\
\hline Juonala et al. ${ }^{40}$ & 2011 & MA & Yes & No & NA & NA & NA & Yes & No & Yes & Yes & NA & Yes & $5 / 7=71 \%$ \\
\hline \multirow[t]{2}{*}{ Total score column $(\%)$} & & & $5 / 5=$ & $3 / 5=$ & $4 / 4=$ & $0 / 4=$ & $1 / 4=$ & $5 / 5=$ & $3 / 5=$ & $3 / 5=$ & $1 / 1=$ & $1 / 4=$ & $4 / 5=$ & \\
\hline & & & $100 \%$ & $60 \%$ & $100 \%$ & $0 \%$ & $25 \%$ & $100 \%$ & $60 \%$ & $60 \%$ & $100 \%$ & $25 \%$ & $80 \%$ & \\
\hline
\end{tabular}

Note: NA is "Not Applicable" is chosen when item is not relevant. Scores are adjusted for NA responses.

A Measurement Tool to Assess Systematic Reviews (AMSTAR) instrument:

1-Type of study design

2-Was a priori design provided?

3-Was there duplicate study selection and data extraction?

4-Was a comprehensive literature search performed?

5 -Was the status of publication (i.e. grey literature) used as an inclusion criterion? *

6-Was a list of studies (included and excluded) provided?

7-Were the characteristics of the included studies provided?

8 -Was the scientific quality of the included studies assessed and documented?

9-Was the scientific quality of the included studies used appropriately in formulating conclusions?

10 -Were the methods used to combine the findings of studies appropriate?

11-Was the likelihood of publication bias assessed?

12 -Was the conflict of interest included?

*This question was modified and is asking if the authors included grey literature as their inclusion criteria. 'Yes' means they did and 'No' means they did not. 
Table 3: Study and participants characteristics:

\begin{tabular}{|c|c|c|c|c|c|c|c|c|c|c|}
\hline $\begin{array}{l}\text { Study-cohort } \\
\text { name }\end{array}$ & $\begin{array}{l}\text { Study } \\
\text { start } \\
\text { year }\end{array}$ & Country & $\begin{array}{l}\text { Study } \\
\text { design* }\end{array}$ & $\begin{array}{l}\mathbf{N} \\
\text { (Baseline) }\end{array}$ & $\begin{array}{l}\text { Age } \\
\text { exposure } \\
\text { assessed }\end{array}$ & $\begin{array}{l}\text { Age } \\
\text { outcome } \\
\text { assessed }\end{array}$ & Exposure & Outcome & $\begin{array}{l}\text { Adj. } \\
\text { adult } \\
\text { BMI }\end{array}$ & Sex \\
\hline $\begin{array}{l}\text { Abraham et al., } \\
1971(\mathrm{HMS})^{95}\end{array}$ & 1923 & USA & $\begin{array}{l}\text { Prospective } \\
\text { Cohort }\end{array}$ & 1087 & $9-12$ & 48 & $\begin{array}{l}\text { Relative over } \\
\text { weight }\end{array}$ & $\begin{array}{l}\text { SBP, } \\
\text { DBP, TC }\end{array}$ & No & Male \\
\hline 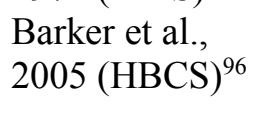 & 1934 & Finland & $\begin{array}{l}\text { Retrospective } \\
\text { longitudinal } \\
\text { study }\end{array}$ & 2003 & $\begin{array}{l}2-11 \text { (age } \\
\text { used in our } \\
\text { study 2) }\end{array}$ & 62 & BMI & $\begin{array}{l}\text { SBP, TC, } \\
\text { TG }\end{array}$ & $\begin{array}{l}\text { Yes } \\
\text { (only) }\end{array}$ & Overall \\
\hline $\begin{array}{l}\text { Berkey et al., } \\
1998 \\
(\text { LSCHD) }\end{array}$ & 1929 & USA & $\begin{array}{l}\text { Prospective } \\
\text { longitudinal } \\
\text { study }\end{array}$ & 67 & 17 & 30 & BMI & SBP & No & $\begin{array}{l}\text { Male } \\
\text { (this } \\
\text { study) } \\
\text { Female }\end{array}$ \\
\hline $\begin{array}{l}\text { Eisenmann et } \\
\text { al., } 2005 \\
(\text { ACLS })^{98}\end{array}$ & 1970 & USA & $\begin{array}{l}\text { Longitudinal, } \\
\text { prospective } \\
\text { epidemiologi } \\
\text { cal study }\end{array}$ & 48 & 16 & 27 & $\begin{array}{l}\text { BMI, WC, } \\
\% \mathrm{BF}\end{array}$ & $\begin{array}{l}\text { SBP, } \\
\text { DBP, TC, } \\
\text { HDL, TG }\end{array}$ & No & Overall \\
\hline $\begin{array}{l}\text { Freedman et al., } \\
2001(\mathrm{BHS})^{4}\end{array}$ & 1973 & USA & $\begin{array}{l}\text { Cross panel } \\
\text { design later } \\
\text { longitudinal } \\
\text { component }\end{array}$ & 2617 & 10 & 27 & $\begin{array}{l}\text { BMI, Triceps } \\
\text { ST }\end{array}$ & $\begin{array}{l}\text { SBP, } \\
\text { DBP, TC, } \\
\text { LDL, } \\
\text { HDL, TG }\end{array}$ & Yes & Overall \\
\hline $\begin{array}{l}\text { Graversen et } \\
\text { al., } 2014 \\
(\text { NFBC 1966) }\end{array}$ & 1966 & Finland & $\begin{array}{l}\text { Population } \\
\text { based cohort }\end{array}$ & 4111 & $\begin{array}{l}2-5 \text { (age used } \\
\text { in our study } \\
5 \text { ) }\end{array}$ & 31 & BMI & $\begin{array}{l}\text { SBP, } \\
\text { DBP, } \\
\text { HDL, TG }\end{array}$ & No & Overall \\
\hline $\begin{array}{l}\text { Gustafsson et } \\
\text { al., } 2011 \\
\text { (NSC) }^{100}\end{array}$ & 1981 & Sweden & $\begin{array}{l}\text { Prospective } \\
\text { cohort study }\end{array}$ & $\begin{array}{l}1083 \\
F=506 \\
M=577\end{array}$ & 16 & $\begin{array}{l}21 \text { (this } \\
\text { study), } \\
30,43\end{array}$ & BMI & SBP, DBP & No & $\begin{array}{l}\text { Male } \\
\text { Female }\end{array}$ \\
\hline $\begin{array}{l}\text { Holland et al., } \\
1993 \\
\text { (NSHD) }^{101}\end{array}$ & 1946 & $\begin{array}{l}\text { England, } \\
\text { Wales, } \\
\text { Scotland }\end{array}$ & $\begin{array}{l}\text { Prospective } \\
\text { longitudinal } \\
\text { study }\end{array}$ & $\begin{array}{l}3332 \text { at } \\
\text { birth }\end{array}$ & $4-7,11-14$ & 36 & BMI & SBP, DBP & No & $\begin{array}{l}\text { Male } \\
\text { Female }\end{array}$ \\
\hline $\begin{array}{l}\text { Kanade et al., } \\
2011 \text { (CBCI })^{102}\end{array}$ & 1979 & India & $\begin{array}{l}\text { Community } \\
\text { base }\end{array}$ & 387 & 3,15 & 24 & BMI & $\begin{array}{l}\text { SBP, } \\
\text { DBP, TG }\end{array}$ & No & Male \\
\hline
\end{tabular}




\begin{tabular}{|c|c|c|c|c|c|c|c|c|c|c|}
\hline $\begin{array}{l}\text { Klumbiene et } \\
\text { al., } 2000 \\
(\mathrm{JHL})^{103}\end{array}$ & 1977 & Lithuania & $\begin{array}{l}\text { prospective } \\
\text { cohort study } \\
\text { Longitudinal } \\
\text { cohort }\end{array}$ & $\begin{array}{l}505 \\
M=217 \\
F=288\end{array}$ & $12-13$ & $32-33$ & $\begin{array}{l}\text { BMI, Triceps } \\
\text { ST, Sub- } \\
\text { scapular ST }\end{array}$ & SBP, DBP & No & $\begin{array}{l}\text { Male } \\
\text { Female }\end{array}$ \\
\hline $\begin{array}{l}\text { Kneisley et al., } \\
1990 \text { (BPS- } \\
\text { TM) }\end{array}$ & 1959 & USA & $\begin{array}{l}\text { Retrospective } \\
\text { Cohort Study }\end{array}$ & $\begin{array}{l}576 \\
M=271 \\
F=305\end{array}$ & 7 & 32 & $\begin{array}{l}\text { Sub-scapular } \\
\text { ST }\end{array}$ & SBP & No & $\begin{array}{l}\text { Male } \\
\text { Female }\end{array}$ \\
\hline $\begin{array}{l}\text { Koziel et al., } \\
2011 \text { (WGS) }\end{array}$ & 1961 & Poland & $\begin{array}{l}\text { Longitudinal } \\
\text { Study }\end{array}$ & $\begin{array}{l}M=124 \\
F=139\end{array}$ & $\begin{array}{l}8,9,10,11 \\
12,13,14 \\
15,16,17,18\end{array}$ & 50 & BMI & SBP & $\begin{array}{l}\text { Yes } \\
\text { (only) }\end{array}$ & $\begin{array}{l}\text { Male } \\
\text { Female }\end{array}$ \\
\hline $\begin{array}{l}\text { Lauer et al., } \\
1993 \text { (MS) }\end{array}$ & 1971 & USA & $\begin{array}{l}\text { Longitudinal } \\
\text { Cohort }\end{array}$ & $\begin{array}{l}M=677 \\
F=748 \\
M=492 \\
F=528\end{array}$ & $\begin{array}{l}\text { 7-8, 9-10, 11- } \\
\text { 12, 13-14, } \\
\text { 15-16, 17-18: } \\
13-14,15-16 \\
17-18\end{array}$ & $\begin{array}{l}\mathbf{2 0}-\mathbf{2 5} \\
26-30\end{array}$ & BMI & SBP, DBP & No & $\begin{array}{l}\text { Male } \\
\text { Female }\end{array}$ \\
\hline $\begin{array}{l}\text { Li et al., } 2007 \\
(\text { BBC- 1958) }\end{array}$ & 1958 & $\begin{array}{l}\text { England, } \\
\text { Wales } \\
\text { and } \\
\text { Scotland }\end{array}$ & $\begin{array}{l}\text { Longitudinal, } \\
\text { Birth Cohort }\end{array}$ & 9297 & $7,11,16$ & $\begin{array}{l}33,45 \\
\text { (this } \\
\text { study) }\end{array}$ & BMI & SBP, DBP & Yes & Overall \\
\hline $\begin{array}{l}\text { Liddle et al., } \\
2012 \\
\text { (MUSP) }^{108}\end{array}$ & 1981 & Australia & $\begin{array}{l}\text { Longitudinal } \\
\text { Birth Cohort } \\
\text { study }\end{array}$ & 1755 & 5 & 21 & $\begin{array}{l}\text { BMI, Triceps } \\
\text { ST }\end{array}$ & SBP, DBP & No & Overall \\
\hline $\begin{array}{l}\text { Lyngdoh et al., } \\
2013 \\
(\text { SCDC) })^{109}\end{array}$ & 1989 & $\begin{array}{l}\text { Seychelle } \\
\text { s }\end{array}$ & $\begin{array}{l}\text { Longitudinal } \\
\text { cohort }\end{array}$ & $\begin{array}{l}390 \\
M=175 \\
F=215\end{array}$ & $12-15$ & $19-20$ & BMI & $\begin{array}{l}\text { SBP, } \\
\text { DBP, } \\
\text { LDL, } \\
\text { HDL, TG }\end{array}$ & Yes & $\begin{array}{l}\text { Overall } \\
\text { Male } \\
\text { Female }\end{array}$ \\
\hline $\begin{array}{l}\text { Miura et al., } \\
2001 \text { (YAJS) }^{110}\end{array}$ & 1965 & Japan & $\begin{array}{l}\text { 20-year FU } \\
\text { data using } \\
\text { record } \\
\text { linkage of a } \\
\text { Birth cohort }\end{array}$ & $\begin{array}{l}M=2198 \\
F=2428\end{array}$ & 3 & 20 & BMI & $\begin{array}{l}\text { SBP, } \\
\text { DBP, TC }\end{array}$ & No & $\begin{array}{l}\text { Male } \\
\text { Female }\end{array}$ \\
\hline
\end{tabular}




\begin{tabular}{|c|c|c|c|c|c|c|c|c|c|c|}
\hline $\begin{array}{l}\text { Pereira et al., } \\
2013 \text { (BBC- } \\
1958)^{111}\end{array}$ & 1958 & $\begin{array}{l}\text { England, } \\
\text { Wales } \\
\text { and } \\
\text { Scotland }\end{array}$ & $\begin{array}{l}\text { Large } \\
\text { Population } \\
\text { based Birth } \\
\text { Cohort }\end{array}$ & $\begin{array}{l}\mathrm{M}=3927 \\
\mathrm{~F}=3897\end{array}$ & $7,11,16$ & $\begin{array}{l}23,33 \\
42,45\end{array}$ & BMI & $\begin{array}{l}\text { TC, LDL, } \\
\text { HDL, TG, } \\
\text { non-HDL }\end{array}$ & No & $\begin{array}{l}\text { Male } \\
\text { Female }\end{array}$ \\
\hline $\begin{array}{l}\text { Porkka et al., } \\
1994 \text { (YFS) }\end{array}$ & 1980 & Finland & $\begin{array}{l}\text { Longitudinal } \\
\text { Cohort }\end{array}$ & 3596 & $\begin{array}{l}3-9,12-18 \\
\text { (this study) }\end{array}$ & $24-30$ & $\begin{array}{l}\text { BMI, Sub- } \\
\text { scapular ST }\end{array}$ & $\begin{array}{l}\text { TC, LDL, } \\
\text { HDL, TG }\end{array}$ & No & $\begin{array}{l}\text { Male } \\
\text { Female }\end{array}$ \\
\hline $\begin{array}{l}\text { Schmidt et al., } \\
2011 \text { (ASHFS- } \\
\text { CDAHS) }\end{array}$ & 1985 & Australia & $\begin{array}{l}\text { Prospective } \\
\text { cohort study }\end{array}$ & 2188 & $7-15$ & $26-32$ & $\begin{array}{l}\text { BMI, WC, } \\
\text { WHR, weight } \\
\text { to height } \\
\text { ratio, sum ST }\end{array}$ & $\begin{array}{l}\text { SBP, } \\
\text { DBP, } \\
\text { HDL, TG }\end{array}$ & No & Overall \\
\hline $\begin{array}{l}\text { Skidmore et al., } \\
2007 \\
(\mathrm{NSHD})^{114}\end{array}$ & 1946 & $\begin{array}{l}\text { England, } \\
\text { Wales } \\
\text { and } \\
\text { Scotland }\end{array}$ & $\begin{array}{l}\text { Prospective } \\
\text { longitudinal } \\
\text { birth cohort } \\
\text { study }\end{array}$ & $\begin{array}{l}5362 \\
\text { births } \\
(2311 \text { for } \\
\text { the } \\
\text { analysis) }\end{array}$ & $2,4,7,11,15$ & 53 & BMI & $\begin{array}{l}\text { TC, LDL, } \\
\text { HDL }\end{array}$ & No & Overall \\
\hline $\begin{array}{l}\text { Weitz et al., } \\
2014 \text { (LS-Mid- } \\
\text { TC) }\end{array}$ & 1966 & $\begin{array}{l}\text { Six } \\
\text { Solomon } \\
\text { Island }\end{array}$ & Longitudinal & $\begin{array}{l}540 \\
M=169 \\
F=219\end{array}$ & $\begin{array}{l}0-5,6-11,12- \\
19(6-11,12- \\
19 \text { for this } \\
\text { study) }\end{array}$ & 25 & $\begin{array}{l}\text { BMI, Sub- } \\
\text { scapular ST }\end{array}$ & $\mathrm{TC}, \mathrm{TG}$ & No & $\begin{array}{l}\text { Male } \\
\text { Female }\end{array}$ \\
\hline $\begin{array}{l}\text { Wright et al., } \\
2001 \\
(\text { NTFCS) }\end{array}$ & 1947 & UK & $\begin{array}{l}\text { Prospective } \\
\text { birth cohort }\end{array}$ & $\begin{array}{l}1142 \text { at } \\
\text { birth, } 2 / 3^{\text {rd }} \\
\text { followed } \\
\text { till age } 15\end{array}$ & 9,13 & 50 & & $\begin{array}{l}\text { SBP, } \\
\text { DBP, TC, } \\
\text { LDL, } \\
\text { HDL, TG }\end{array}$ & Yes & $\begin{array}{l}\text { Male } \\
\text { Female }\end{array}$ \\
\hline \multicolumn{11}{|c|}{$\begin{array}{l}\text { CVD-Cardiovascular Disease; SBP-systolic blood pressure; DBP-diastolic blood pressure; TC-total cholesterol; LDL-low-density lipoprotein } \\
\text { cholesterol; HDL-high-density lipoprotein; non-HDL-non-high-density lipoprotein cholesterol; cholesterol; TG-triglycerides; BMI-body mass } \\
\text { index; WC-waist circumference; WHR-waist to hip ratio; ST-skinfold thickness; BF-body fat; M-male; F-female; USA-United States of America; } \\
\text { UK-United Kingdom; FU- follow up; HMS-Hagerstown Morbidity Study; LSCHD-Longitudinal Study of Child Health and Development; HBCS- } \\
\text { Helsinki Birth Cohort Study; ACLS-Aerobics Center Longitudinal Study; BHS-Bogalusa Heart Study; NSC-Northern Swedish Cohort; NFBC } \\
\text { 1966- Northern Finland Birth Cohort } 1966 \text { Study; NSHD-Medical Research Council National Survey of Health and Development; CBCI- } \\
\text { Community Based Cohort study India; JHL-Study of Juvenile Hypertension in Lithuania; BPS-TM-Blood Pressure Study in Tecumseh, Michigan; } \\
\text { WGS-Wroclaw Growth Study; MS- The Muscatine Study; BBC 1958-British Birth Cohort 1958; MUSP-The Mater-University of Queensland } \\
\text { Study of Pregnancy (MUSP); SCDC-Seychelles Child Development Study; YAJS- Young Adult Japanese Study; YFS-Cardiovascular Risk in } \\
\text { Young Finns; ASHFS-CDAHS-Australian Schools Health and Fitness Survey-Childhood Determinants of Adult Health Study; NSHD-Medical } \\
\text { Research Council National Survey of Health and Development; LS-Mid-TC-Longitudinal Study of the Mid-20th Century; NTFCS-Thousand }\end{array}$} \\
\hline
\end{tabular}


Families Cohort Study

Holland et al., 1993 and Pereira et al., 2013 were not included in the meta-analysis.

\#Kanade et al., 2011-TG could not be used for meta-analysis because the study's main outcome was blood pressure and there was not enough information provided for TG to calculate an effects size.

*Terminologies used by the authors 
Table 4: Changes in primary outcomes using any definition for adiposity

\begin{tabular}{|c|c|c|c|c|c|c|}
\hline Variable & Studies (\#) & Participants (\#) & $\operatorname{Zr}(95 \% \mathrm{CI})$ & $\mathbf{Q}(\mathbf{P})$ & $I^{2}(\%)$ & $95 \%$ PI \\
\hline SBP & 16 & 27487 & $0.11(0.07,0.14)$ & $162.44(<0.001)$ & 90.77 & $-0.03,0.23$ \\
\hline SBP (adjusted) & 6 & 15156 & $-0.13(-0.18,-0.07)$ & $43.05(<0.001)$ & 88.39 & $-0.31,0.01$ \\
\hline DBP & 14 & 27153 & $0.11(0.07,0.14)$ & $135.95(<0.001)$ & 90.44 & $-0.01,0.23$ \\
\hline DBP (adjusted) & 5 & 13356 & $-0.11(-0.17,-0.04)$ & $51.75(<0.001)$ & 92.27 & $-0.37,0.06$ \\
\hline $\mathrm{TC}$ & 8 & 10420 & $0.01(-0.05,0.06)$ & $79.69(<0.001)$ & 91.22 & $-0.16,0.18$ \\
\hline TC (adjusted) & 4 & 7272 & $-0.06(-0.12,0.01)$ & $21.72(<0.001)$ & 86.19 & $-0.32,0.19$ \\
\hline LDL & 5 & 5462 & $0.02(-0.06,0.10)$ & $63.07(<0.001)$ & 93.66 & $-0.25,0.27$ \\
\hline LDL (adjusted) & 3 & 3365 & $-0.08(-0.12,-0.05)$ & $0.25(0.879)$ & 0 & $-0.08,-0.08$ \\
\hline HDL & 8 & 7915 & $-0.06(-0.10,-0.02)$ & $57.22(<0.001)$ & 87.77 & $-0.18,0.06$ \\
\hline HDL (adjusted) & 4 & 5854 & $0.04(-0.08,0.15)$ & $51.65(<0.001)$ & 86.44 & $-0.47,0.47$ \\
\hline TG & 8 & 5919 & $0.08(0.03,0.13)$ & $42.0(<0.001)$ & 83.33 & $-0.05,0.25$ \\
\hline TG (adjusted) & 5 & 5854 & $-0.08(-0.19,0.02)$ & $76.20(<0.001)$ & 94.75 & $-0.39,0.31$ \\
\hline
\end{tabular}


Table 5: Changes in primary outcomes using BMI measures only for the childhood exposure

\begin{tabular}{|c|c|c|c|c|c|}
\hline Variable & $\begin{array}{c}\text { Studies } \\
\text { (\#) }\end{array}$ & $\operatorname{Zr}(95 \%$ CI $)$ & $\mathbf{Q}(\mathbf{P})$ & $I^{2}(\%)$ & 95\% PI \\
\hline SBP & 14 & $0.10(0.06,0.13)$ & $97.56(<0.001)$ & 86.68 & $0,0.23$ \\
\hline DBP & 13 & $0.11(0.07,0.14)$ & $100.19(<0.001)$ & 88.02 & $0,0.24$ \\
\hline $\mathrm{TC}$ & 7 & $0.01(-0.05,0.07)$ & $54.28(<0.001)$ & 88.95 & $-0.17,0.16$ \\
\hline LDL & 5 & $0.02(-0.06,0.10)$ & $63.07(<0.001)$ & 93.66 & $-0.28,0.28$ \\
\hline HDL & 8 & $-0.06(-0.11,-0.01)$ & $39.61(<0.001)$ & 82.33 & $-0.19,0.09$ \\
\hline TG & 7 & $0.10(0.02,0.17)$ & $42.0(<0.001)$ & 83.33 & $-0.13,0.34$ \\
\hline \multicolumn{6}{|c|}{$\begin{array}{l}\text { SBP-systolic blood pressure; DBP-diastolic blood pressure; TC-total cholesterol; LDL-low- } \\
\text { density lipoprotein cholesterol; HDL-high-density lipoprotein; non-HDL-non-high-density } \\
\text { lipoprotein cholesterol; cholesterol; TG-triglycerides. } \\
\text { All the studies that used that adjusted for adult BMI used BMI as the exposure during childhood. } \\
\text { The results of the adjusted analysis are same as in table } 4 .\end{array}$} \\
\hline
\end{tabular}




\section{Figures}

\section{Chapter 4}

Figure 1: Flow diagram for the selection of studies

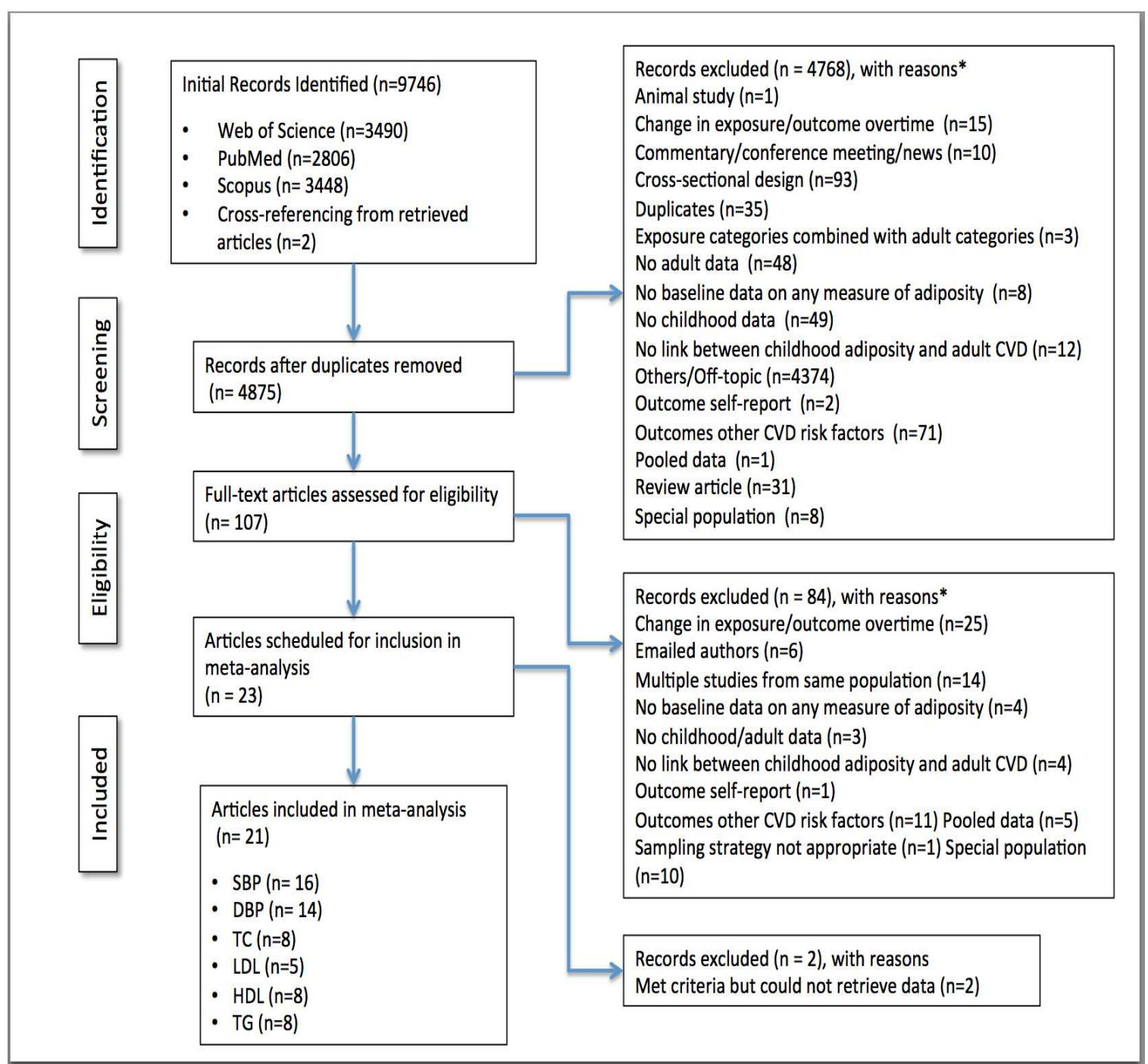

SBP-systolic blood pressure; DBP-diastolic blood pressure; TC-total cholesterol; LDL-lowdensity lipoprotein cholesterol; HDL-high-density lipoprotein; non-HDL-non-high-density lipoprotein cholesterol; cholesterol; TG-triglycerides.

Non-HDL was not analyzed because there were less than 3 studies to pool. 
Figure 2: Risk of bias assessment using STROBE instrument for each study

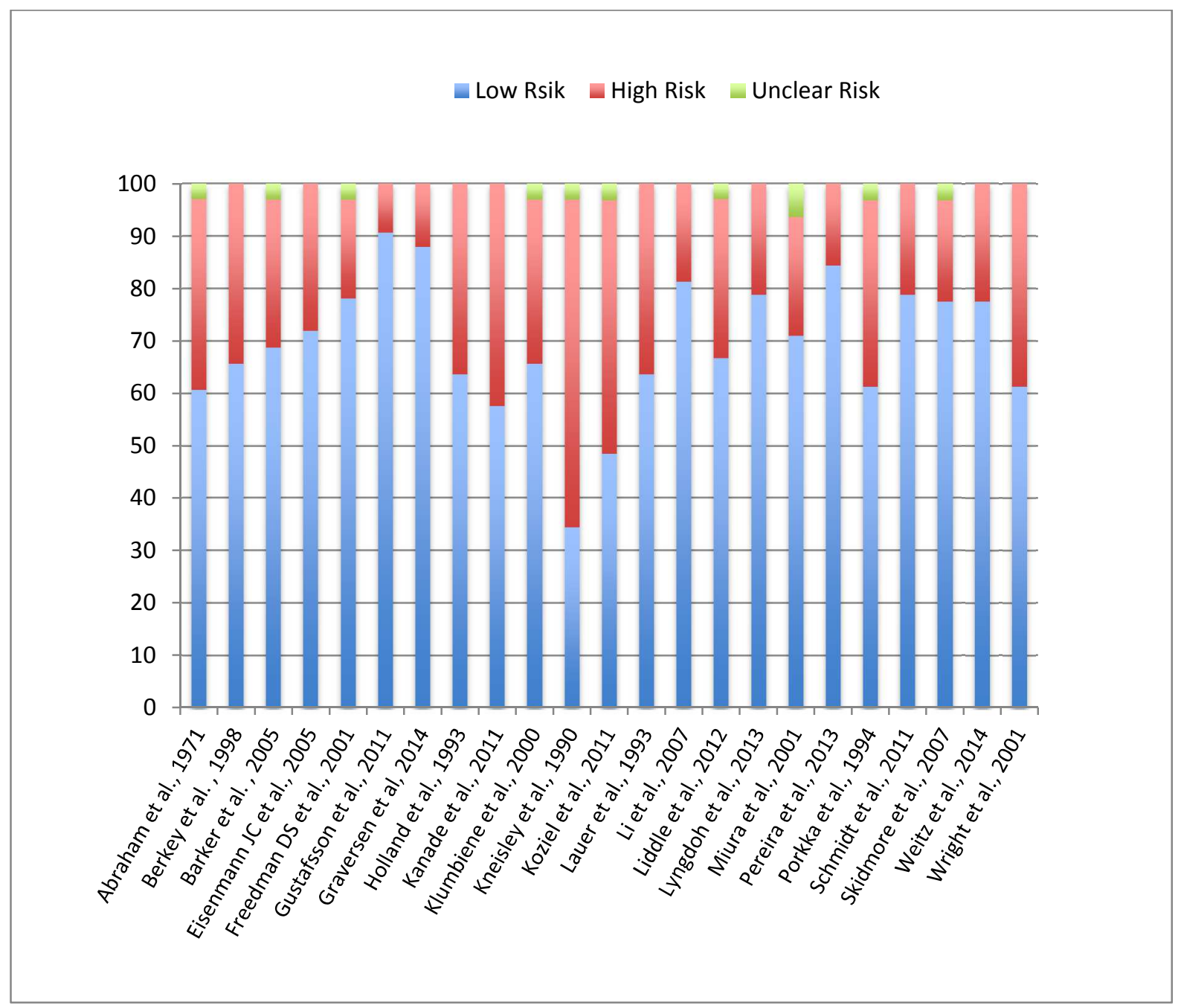

STROBE-Strengthening the Reporting of Observational Studies in Epidemiology (STROBE) consists of a checklist of 22 items related to the title, abstract, introduction, methods, results and discussion sections of the articles. Each study was assessed for the items. NA is "Not Applicable" is chosen when item is not relevant. Scores are adjusted for NA responses. The STROBE checklist is available at: http://strobe-statement.org/index.php?id=available-checklists 
Figure 3. Forest plot for the association between childhood obesity and adult SBP

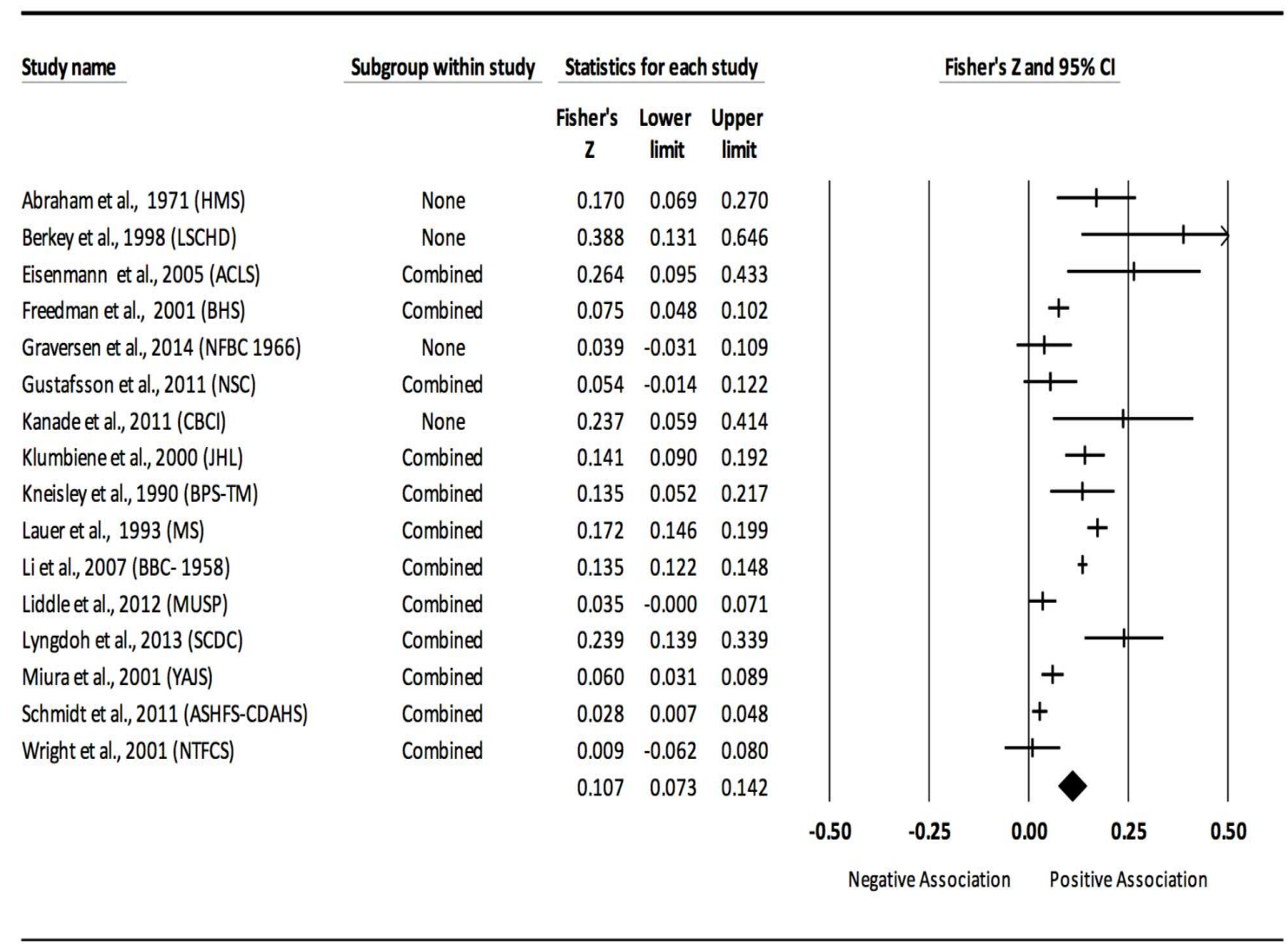

The common metric for the effect size for each study is the Fisher's $r$ to $z$ transformation of the correlation statistics. The vertical lines represent the represent the Fishers $\mathrm{Zr}$ while the left and right extremes of the vertical lines represent the corresponding $95 \%$ CI. The middle of the black diamond represents the overall Fishers $\mathrm{Zr}$ while the left and right extremes of the diamond represent the corresponding $95 \% \mathrm{CI}$. Combined measures represent those studies in which male and female were combined, or different age cohorts from each study were combines, or multiple readings from the same cohort were combined or one study using more than one exposure definition. 
Figure 4. Forest plot for the association between childhood obesity and adult SBP (adjusted for adult BMI)

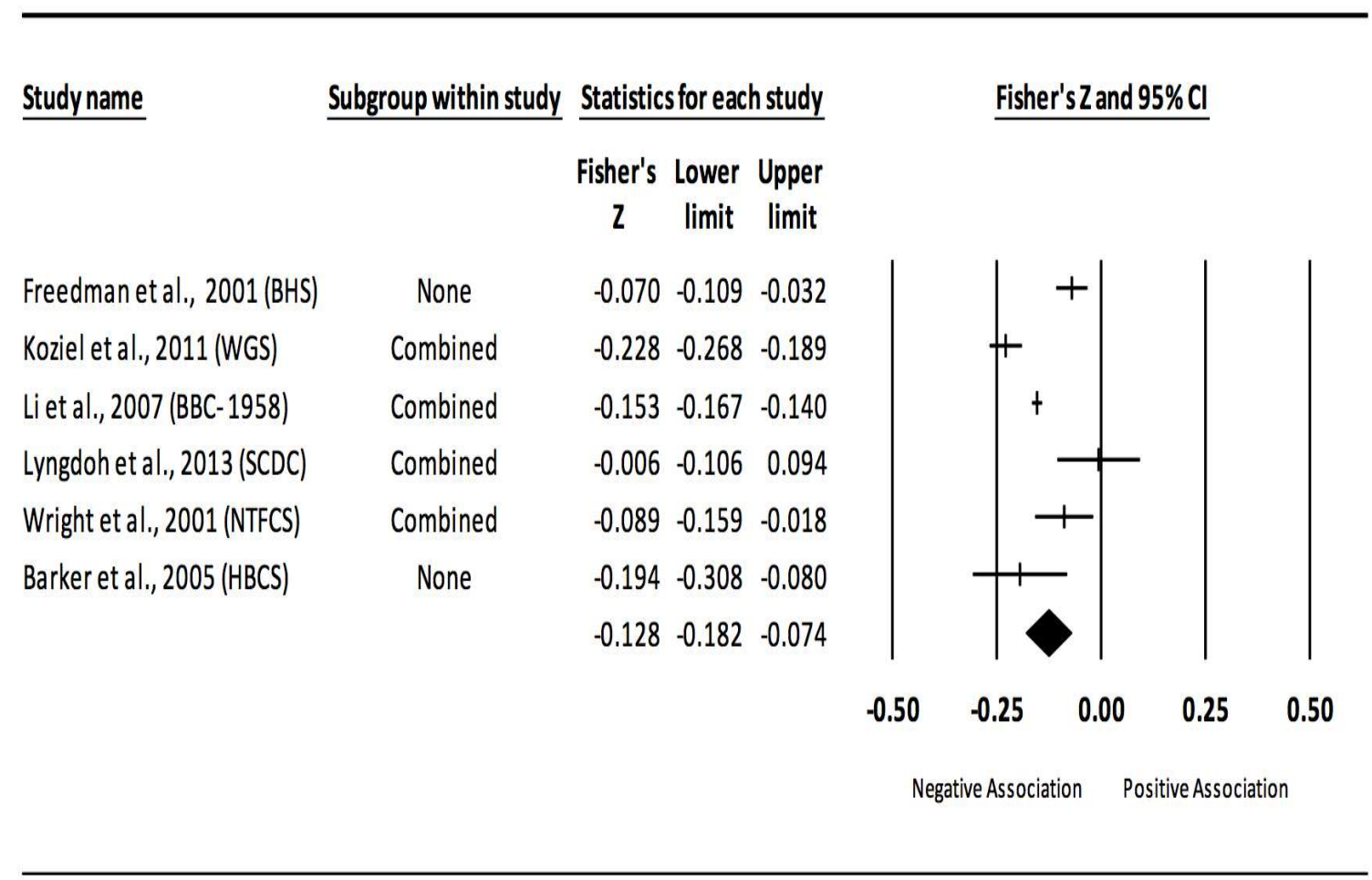

The common metric for the effect size for each study is the Fisher's $r$ to $z$ transformation of the correlation statistics. The vertical lines represent the represent the Fishers $\mathrm{Zr}$ while the left and right extremes of the vertical lines represent the corresponding $95 \%$ CI. The middle of the black diamond represents the overall Fishers $\mathrm{Zr}$ while the left and right extremes of the diamond represent the corresponding 95\% CI. Combined measures represent those studies in which male and female were combined, or different age cohorts from each study were combines, or multiple readings from the same cohort were combined or one study using more than one exposure definition. 
Figure 5. Forest plot for the association between childhood obesity and adult DBP

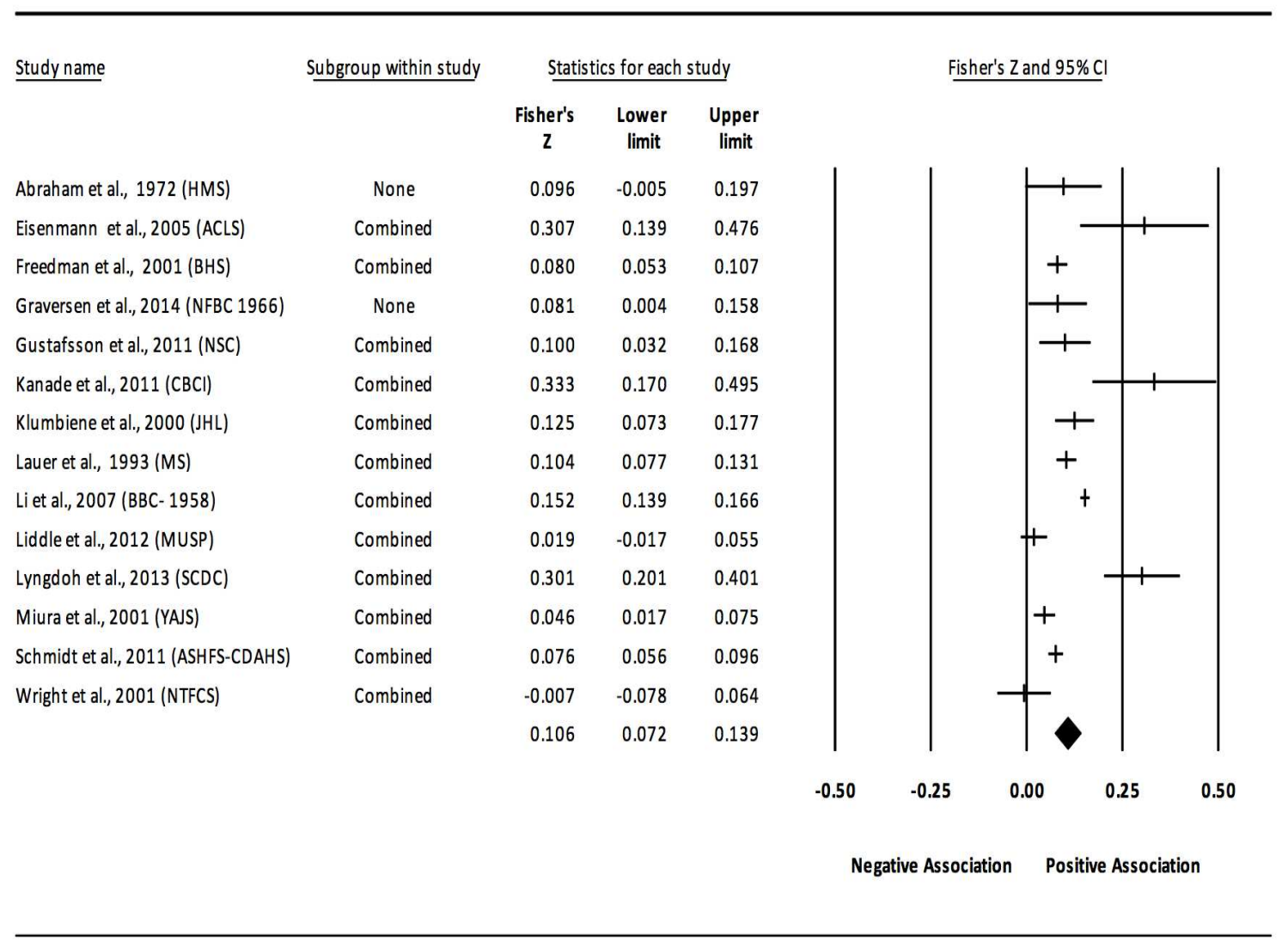

The common metric for the effect size for each study is the Fisher's $r$ to $z$ transformation of the correlation statistics. The vertical lines represent the represent the Fishers $\mathrm{Zr}$ while the left and right extremes of the vertical lines represent the corresponding $95 \% \mathrm{CI}$. The middle of the black diamond represents the overall Fishers $\mathrm{Zr}$ while the left and right extremes of the diamond represent the corresponding $95 \% \mathrm{CI}$. Combined measures represent those studies in which male and female were combined, or different age cohorts from each study were combines, or multiple readings from the same cohort were combined or one study using more than one exposure definition. 
Figure 6. Forest plot for the association between childhood obesity and adult DBP (adjusted for adult BMI)

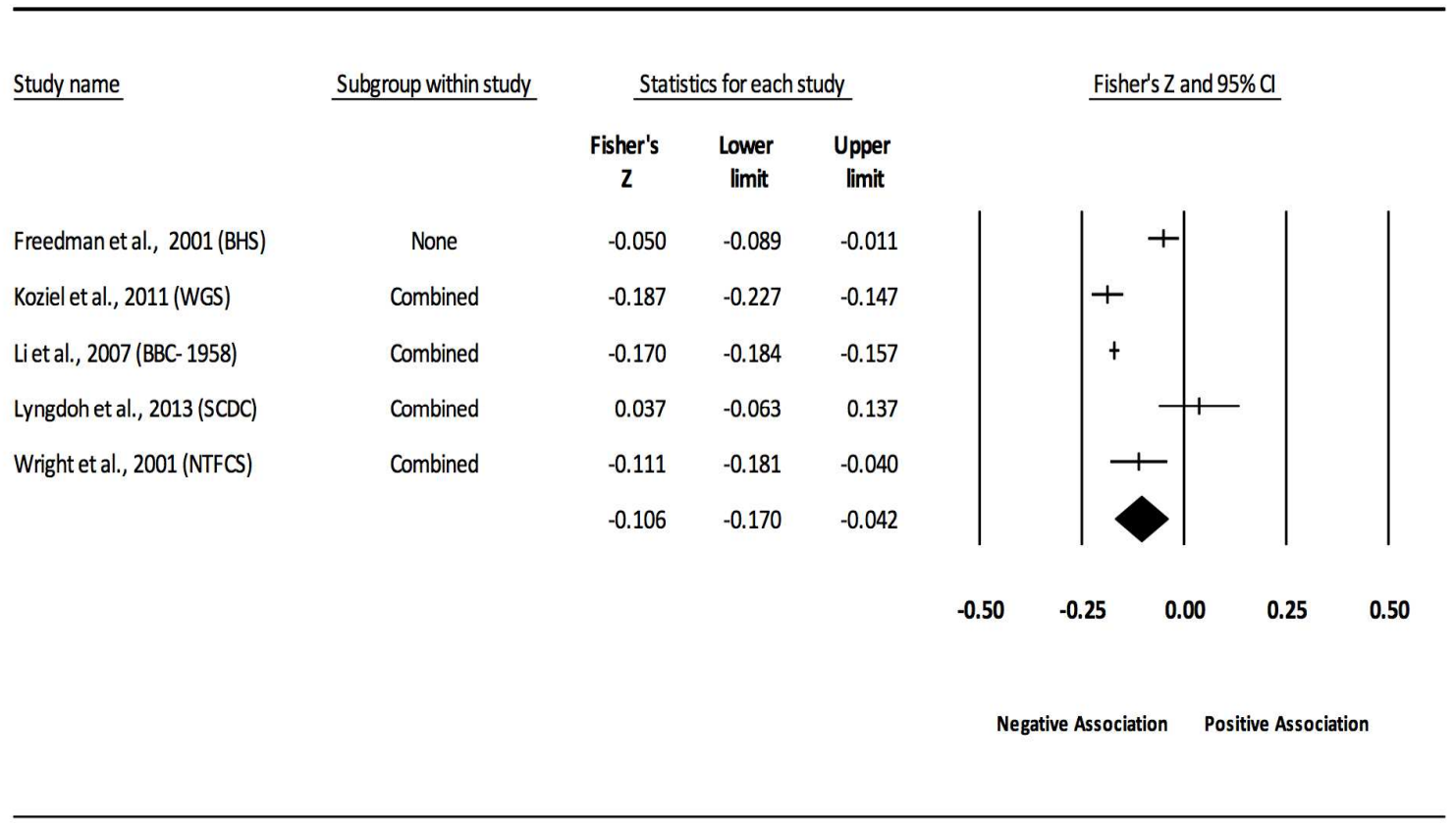

The common metric for the effect size for each study is the Fisher's $r$ to $z$ transformation of the correlation statistics. The vertical lines represent the represent the Fishers $\mathrm{Zr}$ while the left and right extremes of the vertical lines represent the corresponding $95 \%$ CI. The middle of the black diamond represents the overall Fishers $\mathrm{Zr}$ while the left and right extremes of the diamond represent the corresponding 95\% CI. Combined measures represent those studies in which male and female were combined, or different age cohorts from each study were combines, or multiple readings from the same cohort were combined or one study using more than one exposure definition. 
Figure 7. Forest plot for the association between childhood obesity and adult TC

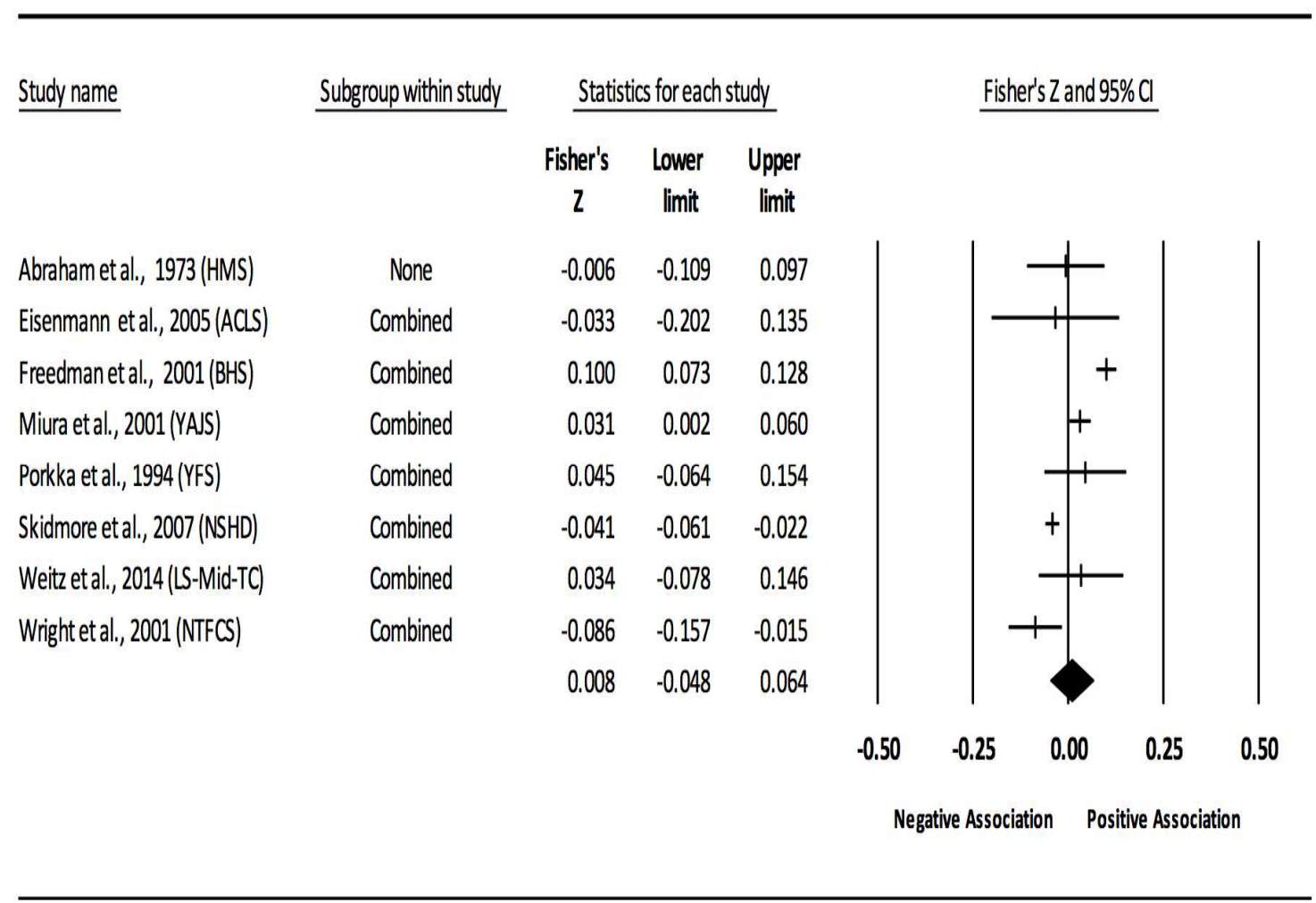

The common metric for the effect size for each study is the Fisher's $r$ to $z$ transformation of the correlation statistics. The vertical lines represent the represent the Fishers $\mathrm{Zr}$ while the left and right extremes of the vertical lines represent the corresponding $95 \% \mathrm{CI}$. The middle of the black diamond represents the overall Fishers $\mathrm{Zr}$ while the left and right extremes of the diamond represent the corresponding 95\% CI. Combined measures represent those studies in which male and female were combined, or different age cohorts from each study were combines, or multiple readings from the same cohort were combined or one study using more than one exposure definition. 
Figure 8. Forest plot for the association between childhood obesity and adult TC (adjusted for adult BMI)

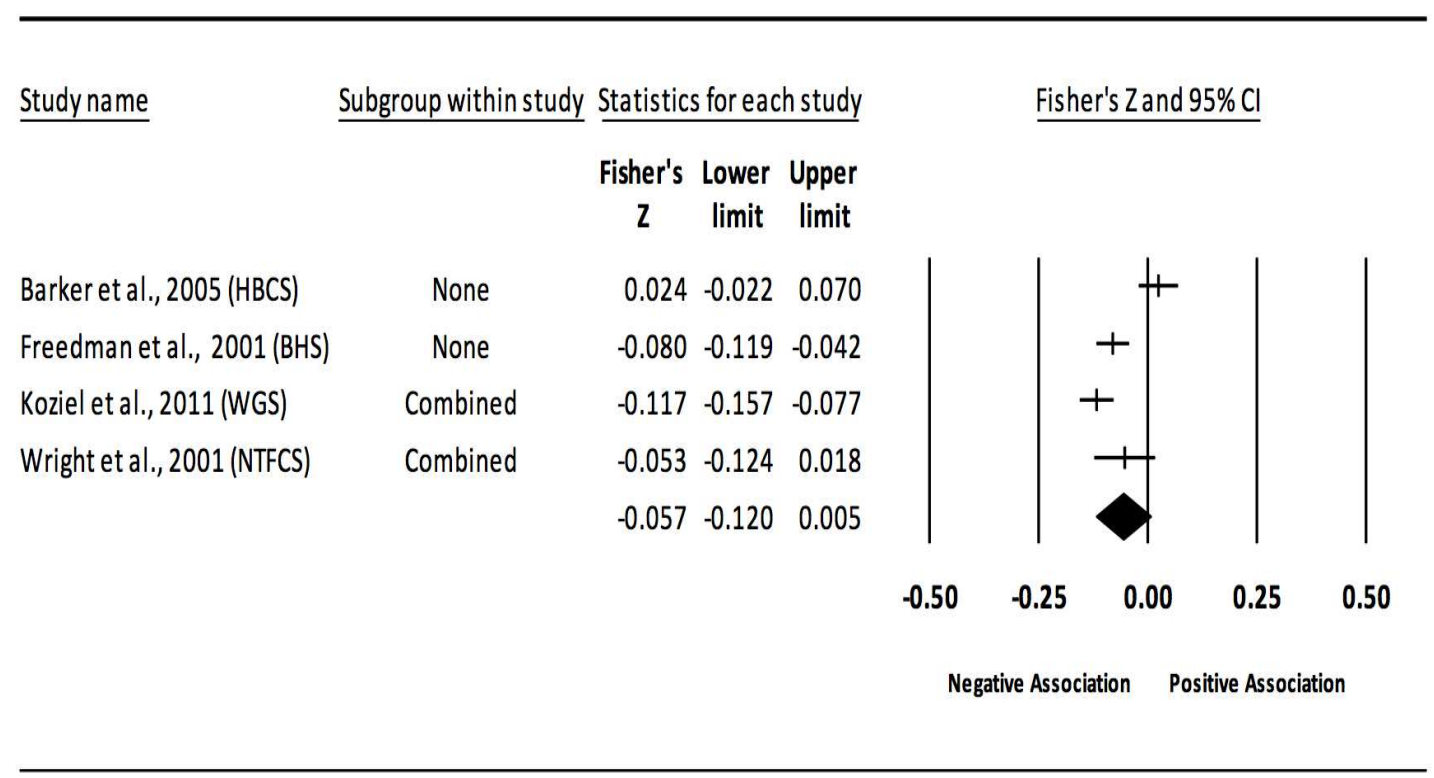

The common metric for the effect size for each study is the Fisher's $r$ to $z$ transformation of the correlation statistics. The vertical lines represent the represent the Fishers $\mathrm{Zr}$ while the left and right extremes of the vertical lines represent the corresponding $95 \% \mathrm{CI}$. The middle of the black diamond represents the overall Fishers $\mathrm{Zr}$ while the left and right extremes of the diamond represent the corresponding 95\%CI. Combined measures represent those studies in which male and female were combined, or different age cohorts from each study were combines, or multiple readings from the same cohort were combined or one study using more than one exposure definition. 
Figure 9. Forest plot for the association between childhood obesity and adult LDL

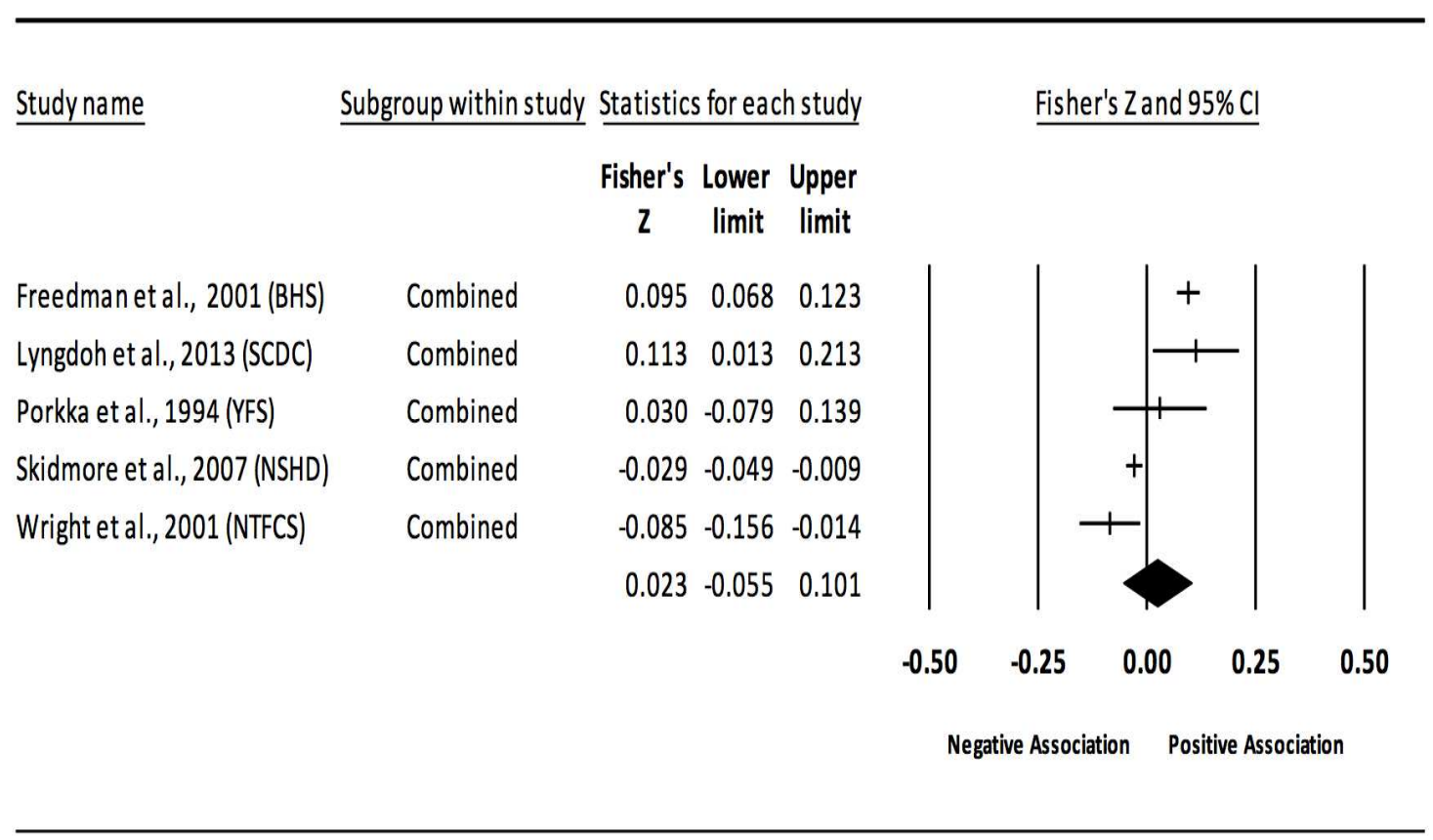

The common metric for the effect size for each study is the Fisher's $r$ to $z$ transformation of the correlation statistics. The vertical lines represent the represent the Fishers $\mathrm{Zr}$ while the left and right extremes of the vertical lines represent the corresponding $95 \% \mathrm{CI}$. The middle of the black diamond represents the overall Fishers $\mathrm{Zr}$ while the left and right extremes of the diamond represent the corresponding 95\%CI. Combined measures represent those studies in which male and female were combined, or different age cohorts from each study were combines, or multiple readings from the same cohort were combined or one study using more than one exposure definition. 
Figure 10. Forest plot for the association between childhood obesity and adult LDL (adjusted for adult BMI)

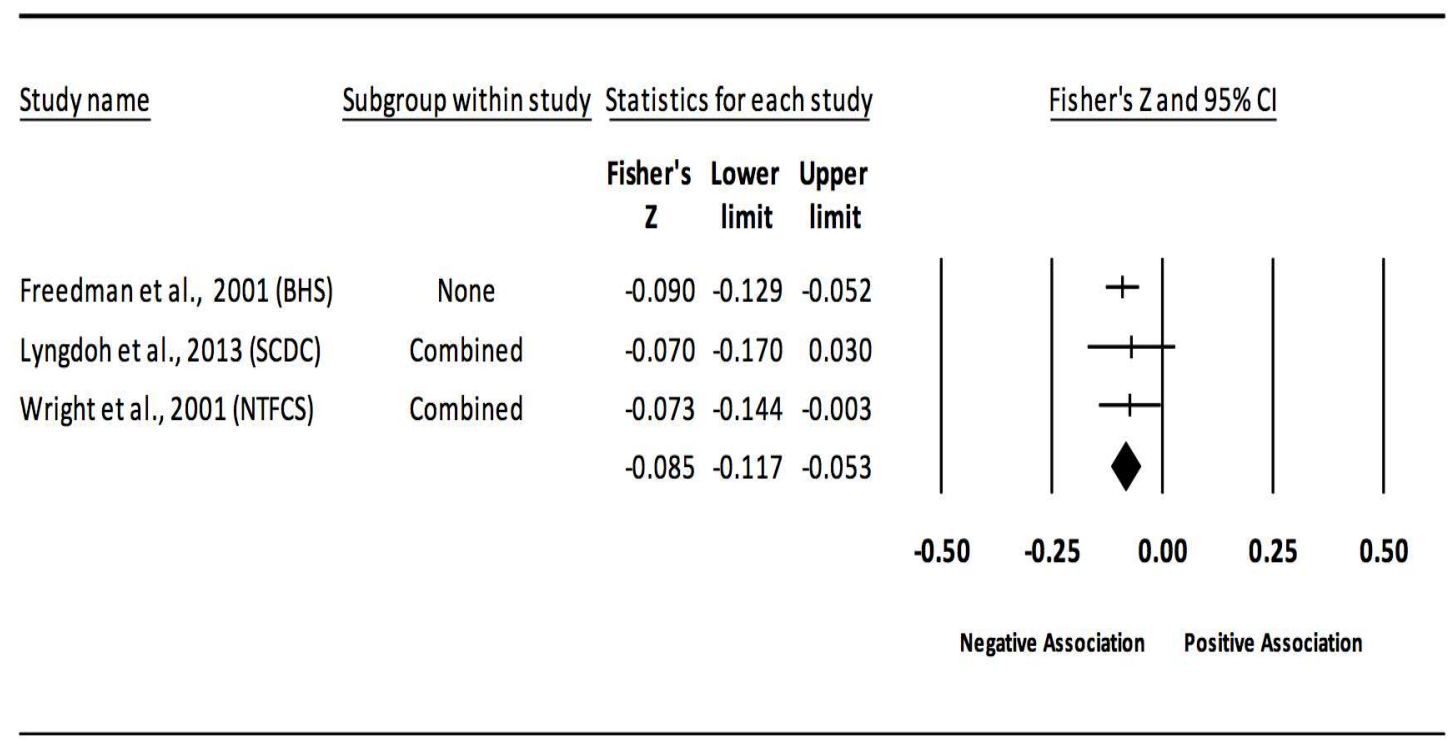

The common metric for the effect size for each study is the Fisher's $r$ to $z$ transformation of the correlation statistics. The vertical lines represent the represent the Fishers $\mathrm{Zr}$ while the left and right extremes of the vertical lines represent the corresponding $95 \% \mathrm{CI}$. The middle of the black diamond represents the overall Fishers $\mathrm{Zr}$ while the left and right extremes of the diamond represent the corresponding 95\%CI. Combined measures represent those studies in which male and female were combined, or different age cohorts from each study were combines, or multiple readings from the same cohort were combined or one study using more than one exposure definition. 
Figure 11. Forest plot for the association between childhood obesity and adult HDL

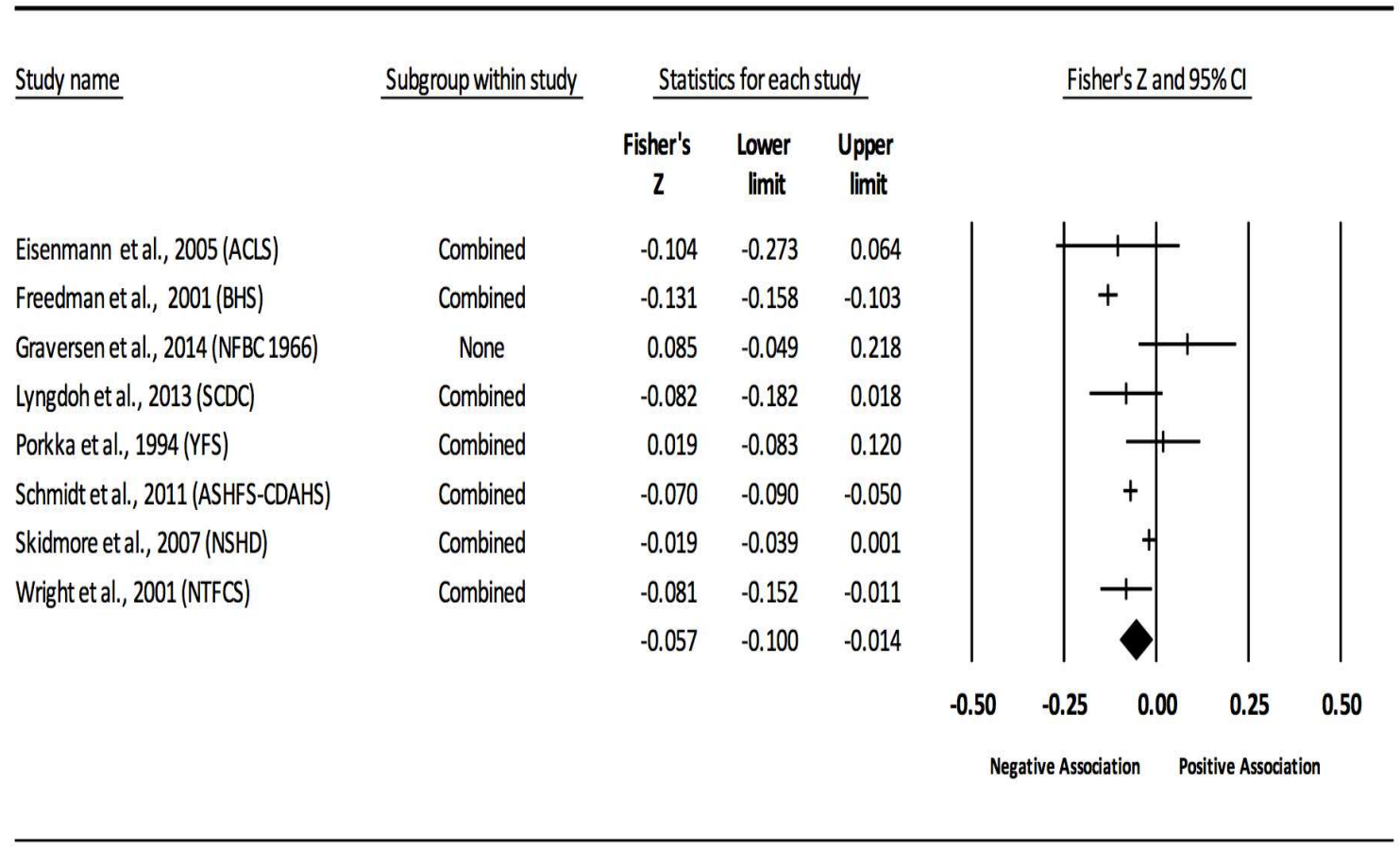

The common metric for the effect size for each study is the Fisher's $r$ to $\mathrm{z}$ transformation of the correlation statistics. The vertical lines represent the represent the Fishers $\mathrm{Zr}$ while the left and right extremes of the vertical lines represent the corresponding 95\% CI. The middle of the black diamond represents the overall Fishers $\mathrm{Zr}$ while the left and right extremes of the diamond represent the corresponding $95 \% \mathrm{CI}$. Combined measures represent those studies in which male and female were combined, or different age cohorts from each study were combines, or multiple readings from the same cohort were combined or one study using more than one exposure definition. 
Figure 12. Forest plot for the association between childhood obesity and adult HDL (adjusted for adult BMI)

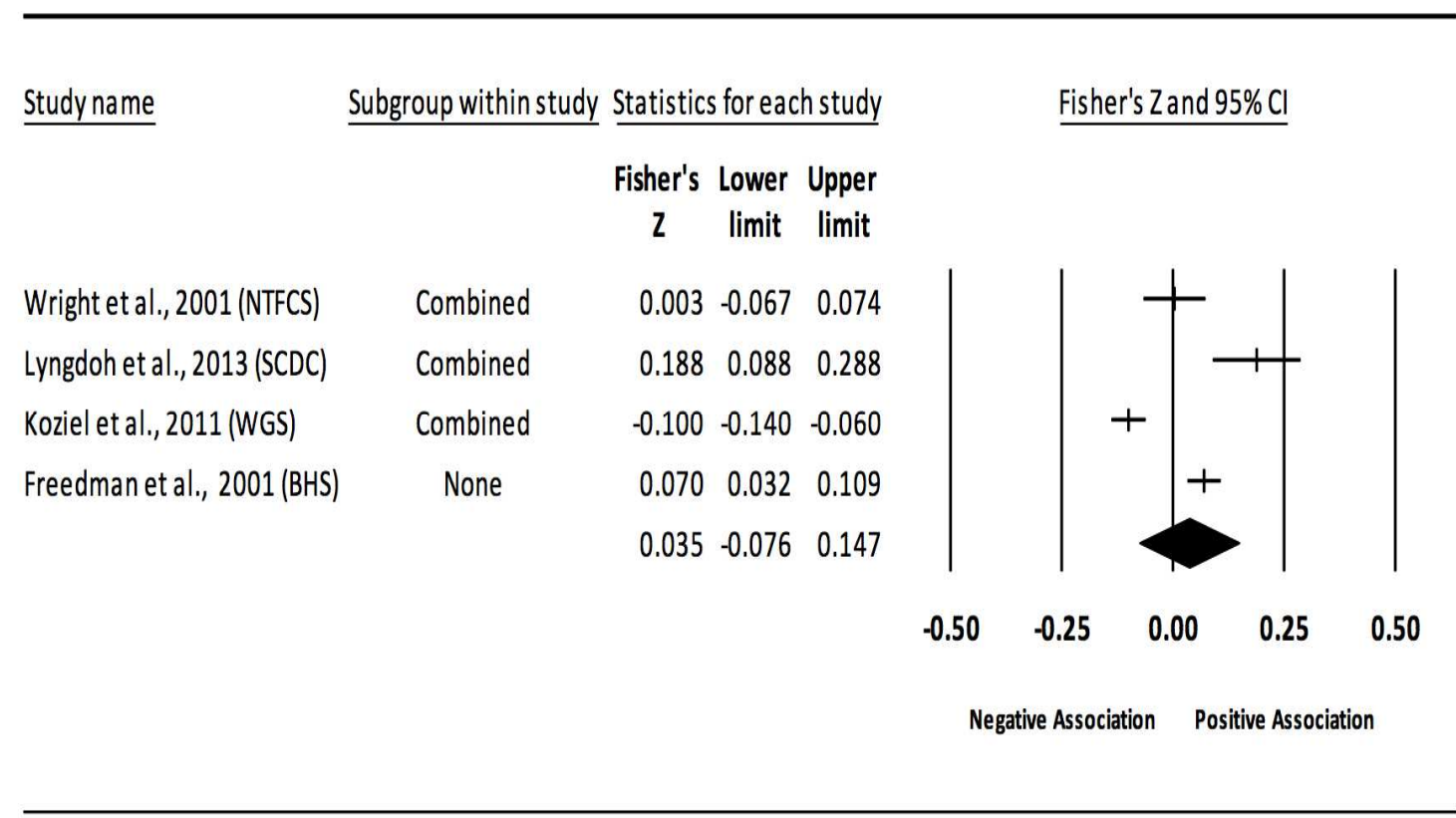

The common metric for the effect size for each study is the Fisher's $r$ to $z$ transformation of the correlation statistics. The vertical lines represent the represent the Fishers $\mathrm{Zr}$ while the left and right extremes of the vertical lines represent the corresponding 95\% CI. The middle of the black diamond represents the overall Fishers $\mathrm{Zr}$ while the left and right extremes of the diamond represent the corresponding $95 \% \mathrm{CI}$. Combined measures represent those studies in which male and female were combined, or different age cohorts from each study were combines, or multiple readings from the same cohort were combined or one study using more than one exposure definition. 
Figure 13. Forest plot for the association between childhood obesity and adult TG

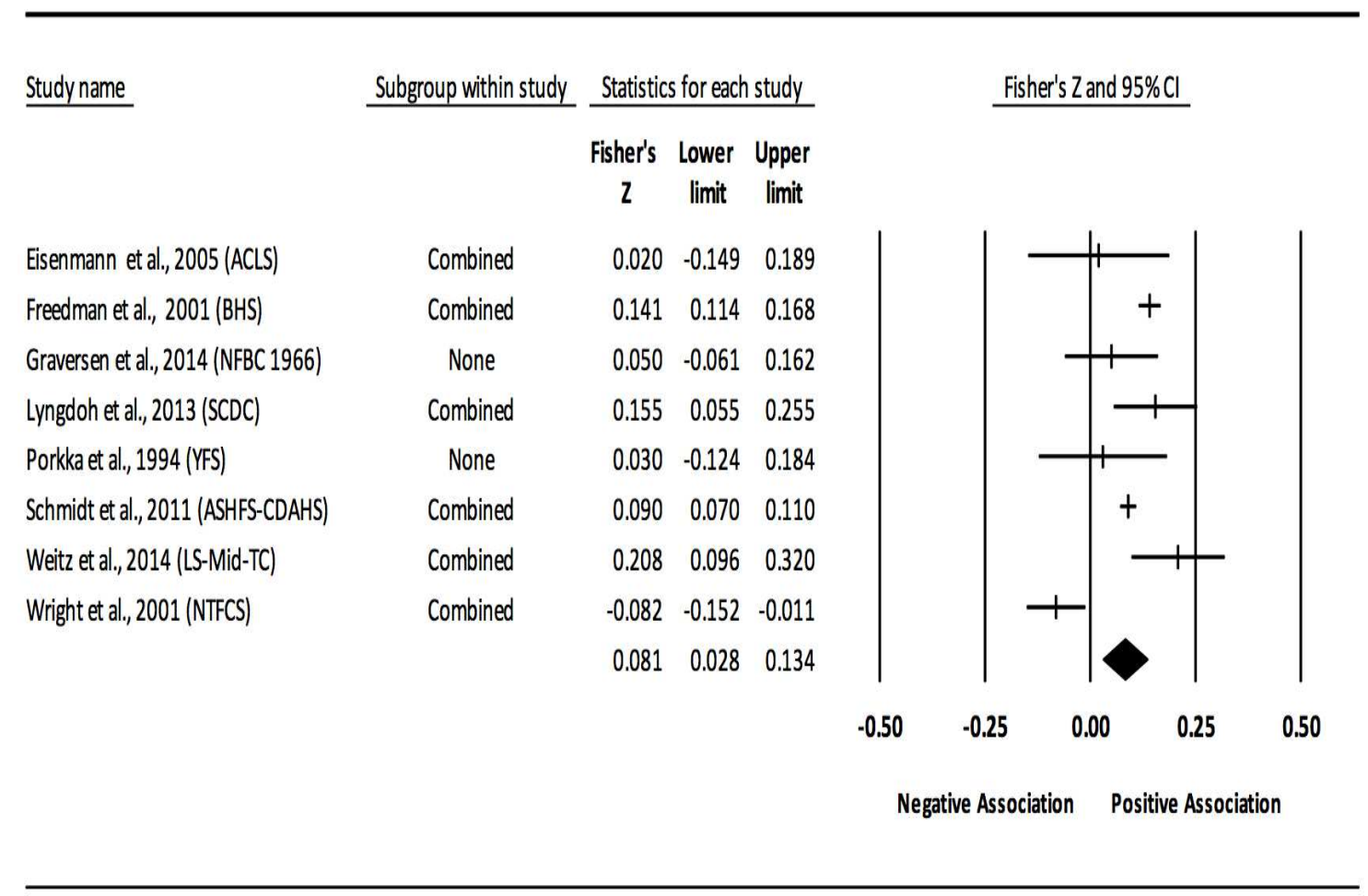

The common metric for the effect size for each study is the Fisher's $r$ to $z$ transformation of the correlation statistics. The vertical lines represent the represent the Fishers $\mathrm{Zr}$ while the left and right extremes of the vertical lines represent the corresponding 95\% CI. The middle of the black diamond represents the overall Fishers $\mathrm{Zr}$ while the left and right extremes of the diamond represent the corresponding 95\%CI. Combined measures represent those studies in which male and female were combined, or different age cohorts from each study were combines, or multiple readings from the same cohort were combined or one study using more than one exposure definition. 
Figure 14. Forest plot for the association between childhood obesity and adult TG (adjusted for adult BMI)

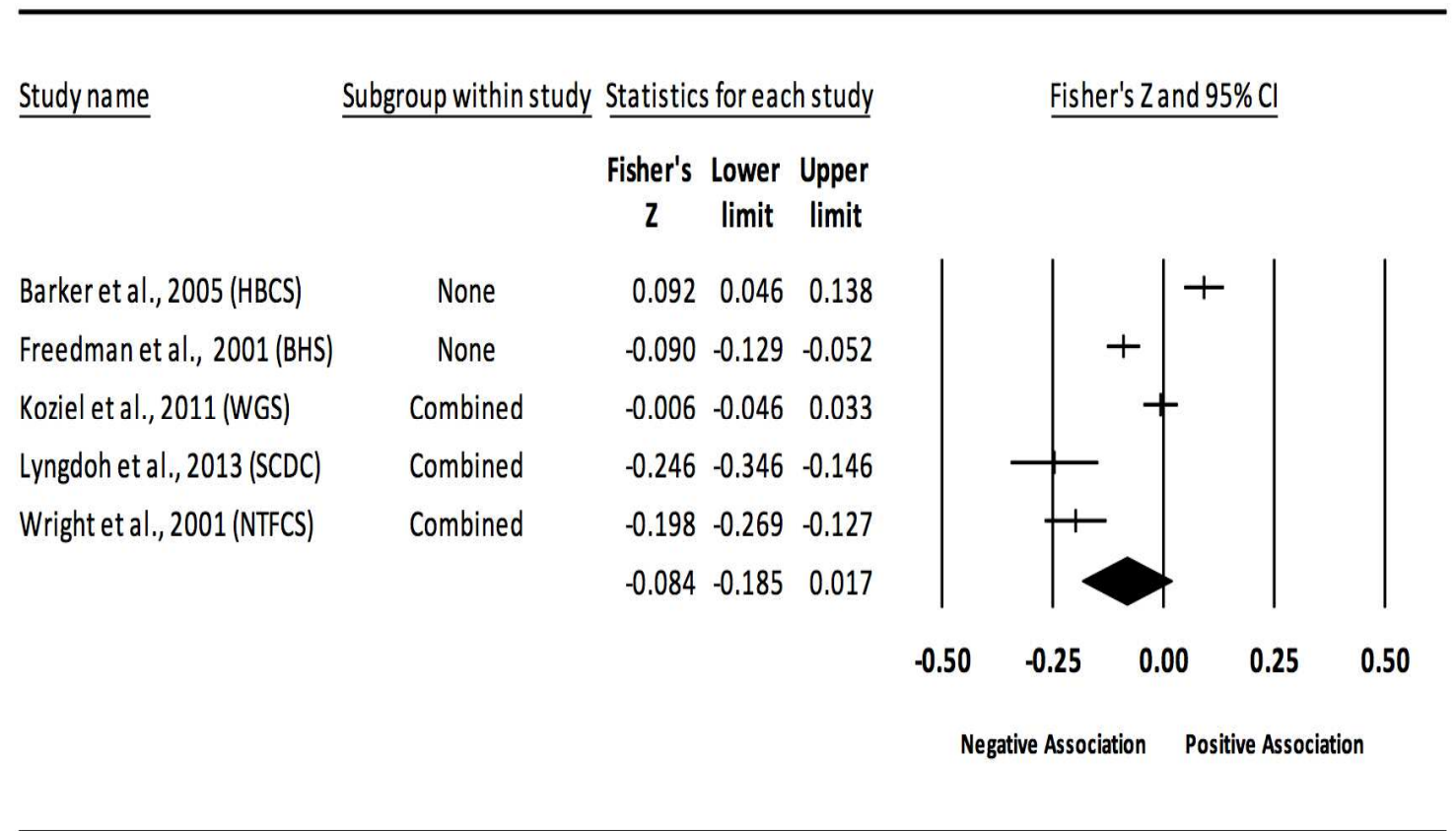

The common metric for the effect size for each study is the Fisher's $r$ to $z$ transformation of the correlation statistics. The vertical lines represent the represent the Fishers $\mathrm{Zr}$ while the left and right extremes of the vertical lines represent the corresponding $95 \% \mathrm{CI}$. The middle of the black diamond represents the overall Fishers $\mathrm{Zr}$ while the left and right extremes of the diamond represent the corresponding 95\%CI. Combined measures represent those studies in which male and female were combined, or different age cohorts from each study were combines, or multiple readings from the same cohort were combined or one study using more than one exposure definition. 


\section{Additional Files}

\section{Chapter 4}

Additional File 1- Search Strategy for Databases (June 5, 2015)

PubMed Database Search:

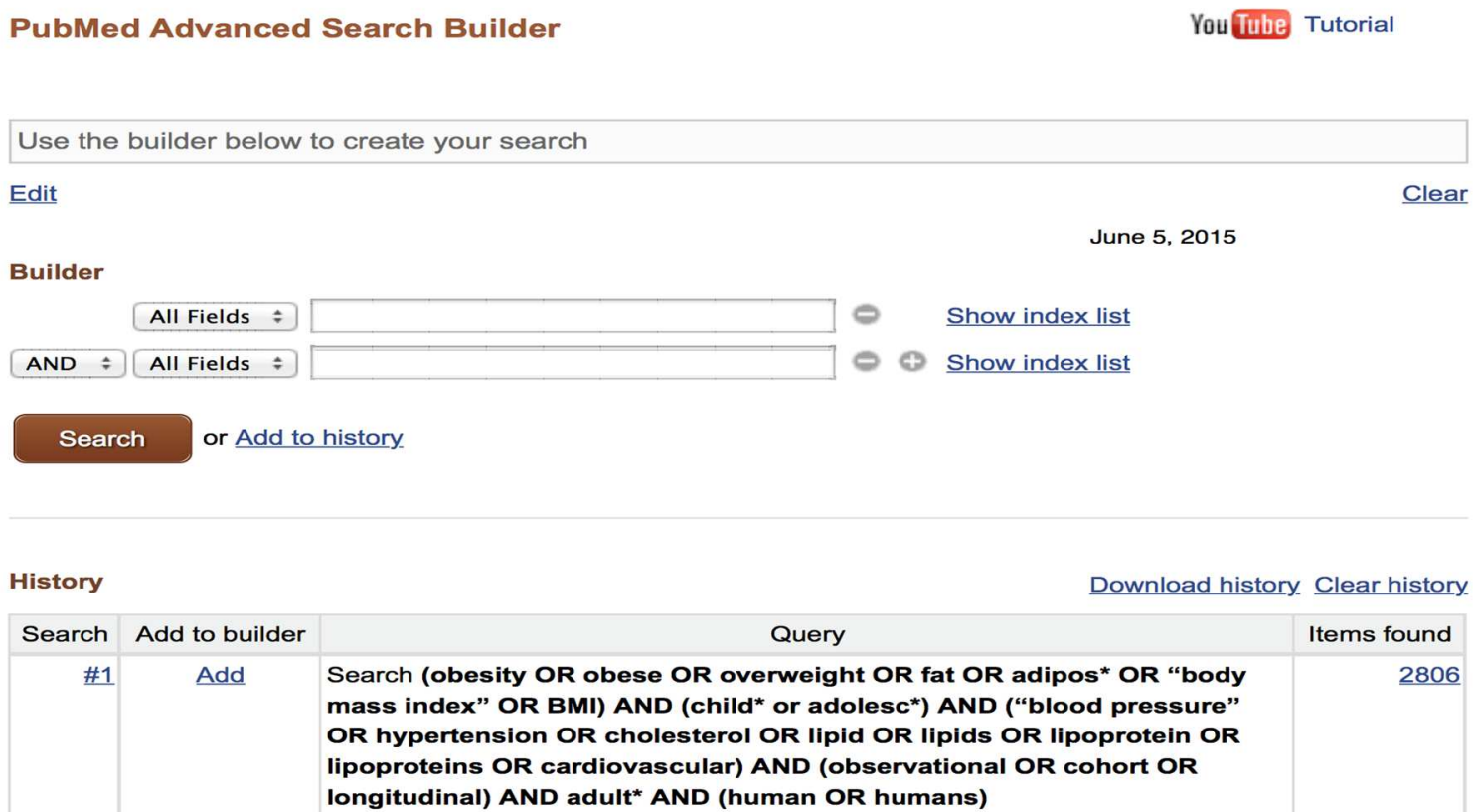

Web of Science Database Search

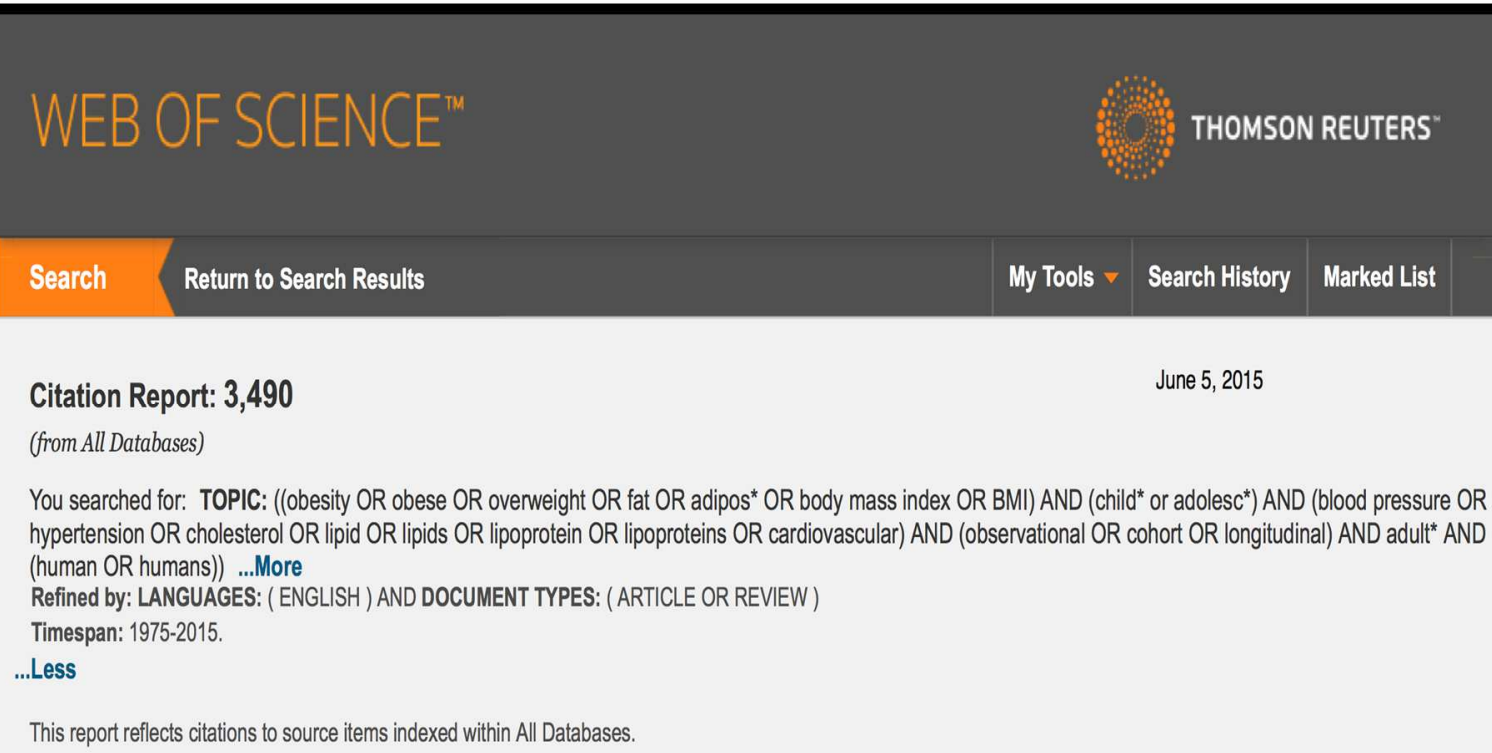

Citation Report: 3,490

(from All Databases)

You searched for: TOPIC: ((obesity OR obese OR overweight OR fat OR adipos* OR body mass index OR BMI) AND (child* or adolesc*) AND (blood pressure OR hypertension OR cholesterol OR lipid OR lipids OR lipoprotein OR lipoproteins OR cardiovascular) AND (observational OR cohort OR longitudinal) AND adult* AND (human OR humans)) ....More

Refined by: LANGUAGES: (ENGLISH ) AND DOCUMENT TYPES: ( ARTICLE OR REVIEW)

Timespan: 1975-2015.

...Less

This report reflects citations to source items indexed within All Databases. 
Scopus Database Search

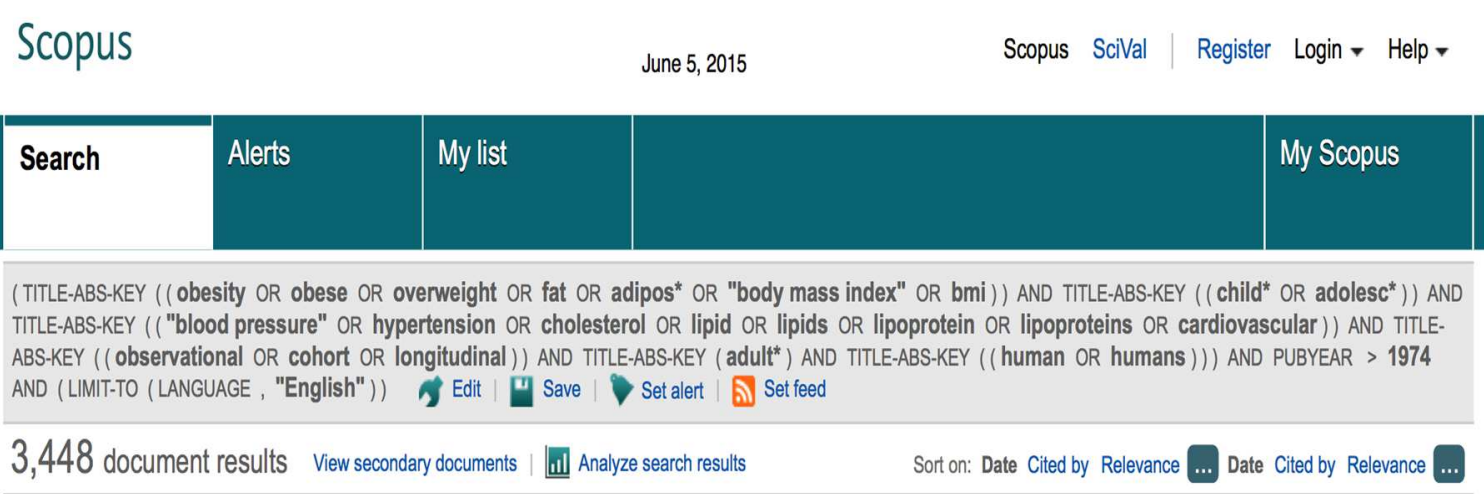

Search Strategy for Databases (February 2, 2015) to find existing systematic reviews and metaanalysis on the topic:

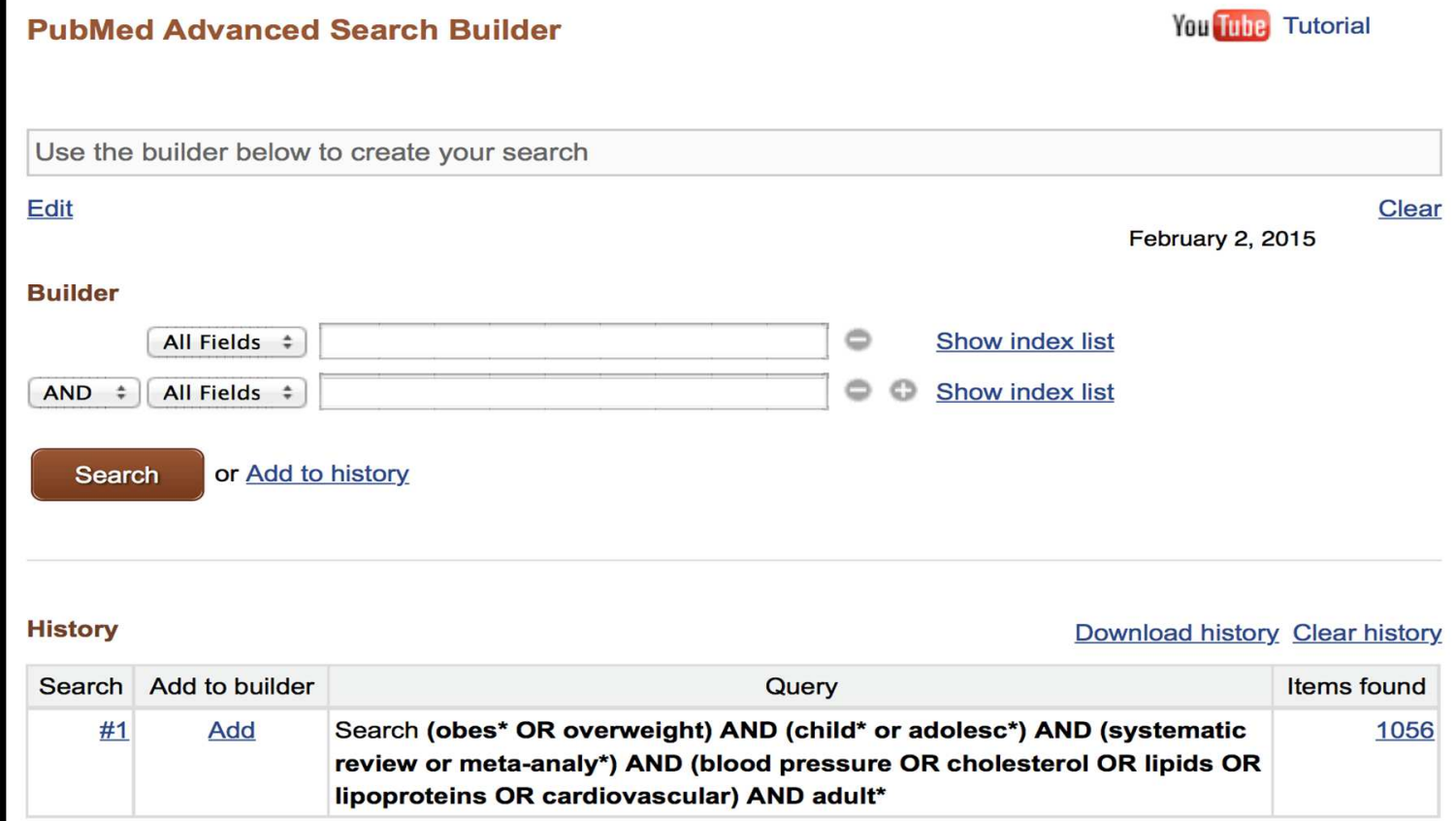


Additional file 2: List of excluded studies with reasons.

Approximately 5000 citations

Available upon request: Contact amnaumer@gmail.com 


\section{Additional file 3: Strobe Checklist}

\begin{tabular}{|c|c|c|c|c|c|c|c|c|c|c|c|c|c|c|c|c|c|}
\hline STROBE items & $\begin{array}{c}1 \\
\text { (a) }\end{array}$ & $\begin{array}{c}1 \\
\text { (b) }\end{array}$ & 2 & 3 & 4 & 5 & 6 (a) & $\begin{array}{c}6 \\
\text { (b) }\end{array}$ & 7 & 8 & 9 & 10 & 11 & $\begin{array}{l}12 \\
\text { (a) }\end{array}$ & $\begin{array}{l}12 \\
\text { (b) }\end{array}$ & $\begin{array}{l}12 \\
(' c)\end{array}$ & $\begin{array}{l}12 \\
\text { (d) }\end{array}$ \\
\hline Abraham et al., 1971 & $\mathrm{H}$ & $\mathrm{H}$ & $\mathrm{L}$ & $\mathrm{L}$ & $\mathrm{L}$ & $\mathrm{L}$ & $\mathrm{L}$ & NA & $\mathrm{L}$ & $\mathrm{L}$ & $\mathrm{L}$ & $\mathrm{L}$ & $\mathrm{L}$ & $\mathrm{H}$ & $\mathrm{L}$ & $\mathrm{H}$ & $\mathrm{L}$ \\
\hline Berkey et al., 1998 & $\mathrm{~L}$ & $\mathrm{~L}$ & $\mathrm{~L}$ & $\mathrm{~L}$ & $\mathrm{~L}$ & $\mathrm{~L}$ & $\mathrm{H}$ & NA & $\mathrm{L}$ & $\mathrm{L}$ & $\mathrm{H}$ & $\mathrm{H}$ & $\mathrm{H}$ & $\mathrm{L}$ & $\mathrm{H}$ & $\mathrm{H}$ & $\mathrm{H}$ \\
\hline Barker et al., 2005 & $\mathrm{~L}$ & $\mathrm{~L}$ & $\mathrm{~L}$ & $\mathrm{~L}$ & $\mathrm{~L}$ & $\mathrm{~L}$ & $\mathrm{~L}$ & NA & $\mathrm{L}$ & $\mathrm{L}$ & $\mathrm{H}$ & $\mathrm{L}$ & $\mathrm{H}$ & $\mathrm{L}$ & $\mathrm{L}$ & $\mathrm{H}$ & $\mathrm{H}$ \\
\hline $\begin{array}{l}\text { Eisenmann JC et al., } \\
2005\end{array}$ & $\mathrm{~L}$ & $\mathrm{~L}$ & $\mathrm{~L}$ & $\mathrm{~L}$ & $\mathrm{~L}$ & $\mathrm{~L}$ & $\mathrm{~L}$ & NA & $\mathrm{L}$ & $\mathrm{L}$ & $\mathrm{H}$ & $\mathrm{L}$ & $\mathrm{L}$ & $\mathrm{L}$ & $\mathrm{L}$ & $\mathrm{H}$ & $\mathrm{H}$ \\
\hline $\begin{array}{l}\text { Freedman DS et al., } \\
2001\end{array}$ & $\mathrm{~L}$ & $\mathrm{~L}$ & $\mathrm{~L}$ & $\mathrm{~L}$ & $\mathrm{~L}$ & $\mathrm{~L}$ & $\mathrm{~L}$ & NA & $\mathrm{L}$ & $\mathrm{L}$ & $\mathrm{H}$ & $\mathrm{L}$ & $\mathrm{L}$ & $\mathrm{L}$ & $\mathrm{L}$ & $\mathrm{H}$ & $\mathrm{H}$ \\
\hline $\begin{array}{l}\text { Gustafsson et al., } \\
2011\end{array}$ & $\mathrm{~L}$ & $\mathrm{~L}$ & $\mathrm{~L}$ & $\mathrm{~L}$ & $\mathrm{~L}$ & $\mathrm{~L}$ & $\mathrm{~L}$ & NA & $\mathrm{L}$ & $\mathrm{L}$ & $\mathrm{L}$ & $\mathrm{L}$ & $\mathrm{L}$ & $\mathrm{L}$ & $\mathrm{L}$ & $\mathrm{L}$ & $\mathrm{L}$ \\
\hline Graversen et al, 2014 & $\mathrm{~L}$ & $\mathrm{~L}$ & $\mathrm{~L}$ & $\mathrm{~L}$ & $\mathrm{~L}$ & $\mathrm{~L}$ & $\mathrm{~L}$ & NA & $\mathrm{L}$ & $\mathrm{L}$ & $\mathrm{H}$ & $\mathrm{L}$ & $\mathrm{L}$ & $\mathrm{L}$ & $\mathrm{L}$ & $\mathrm{H}$ & $\mathrm{H}$ \\
\hline Holland et al., 1993 & $\mathrm{~L}$ & $\mathrm{~L}$ & $\mathrm{~L}$ & $\mathrm{~L}$ & $\mathrm{~L}$ & $\mathrm{~L}$ & $\mathrm{~L}$ & NA & $\mathrm{L}$ & $\mathrm{L}$ & $\mathrm{L}$ & $\mathrm{L}$ & $\mathrm{L}$ & $\mathrm{H}$ & $\mathrm{H}$ & $\mathrm{L}$ & $\mathrm{H}$ \\
\hline Kanade et al., 2011 & $\mathrm{~L}$ & $\mathrm{~L}$ & $\mathrm{~L}$ & $\mathrm{~L}$ & $\mathrm{~L}$ & $\mathrm{~L}$ & $\mathrm{~L}$ & NA & $\mathrm{L}$ & $\mathrm{L}$ & $\mathrm{H}$ & $\mathrm{L}$ & $\mathrm{L}$ & $\mathrm{H}$ & $\mathrm{H}$ & $\mathrm{H}$ & $\mathrm{H}$ \\
\hline $\begin{array}{l}\text { Klumbiene et al., } \\
2000\end{array}$ & $\mathrm{~L}$ & $\mathrm{~L}$ & $\mathrm{~L}$ & $\mathrm{~L}$ & $\mathrm{~L}$ & $\mathrm{~L}$ & $\mathrm{~L}$ & NA & $\mathrm{L}$ & $\mathrm{L}$ & $\mathrm{H}$ & $\mathrm{L}$ & $\mathrm{L}$ & $\mathrm{L}$ & $\mathrm{L}$ & $\mathrm{H}$ & $\mathrm{H}$ \\
\hline Kneisley et al., 1990 & $\mathrm{H}$ & $\mathrm{L}$ & $\mathrm{H}$ & $\mathrm{L}$ & $\mathrm{H}$ & $\mathrm{H}$ & $\mathrm{L}$ & NA & UN & $\mathrm{H}$ & $\mathrm{H}$ & $\mathrm{L}$ & $\mathrm{L}$ & $\mathrm{H}$ & $\mathrm{H}$ & $\mathrm{H}$ & $\mathrm{H}$ \\
\hline Koziel et al., 2011 & $\mathrm{~L}$ & $\mathrm{~L}$ & $\mathrm{~L}$ & $\mathrm{~L}$ & $\mathrm{~L}$ & $\mathrm{~L}$ & $\mathrm{H}$ & NA & $\mathrm{L}$ & $\mathrm{L}$ & $\mathrm{H}$ & $\mathrm{UN}$ & $\mathrm{L}$ & $\mathrm{L}$ & $\mathrm{H}$ & $\mathrm{H}$ & $\mathrm{H}$ \\
\hline Lauer et al., 1993 & $\mathrm{H}$ & $\mathrm{H}$ & $\mathrm{L}$ & $\mathrm{L}$ & $\mathrm{L}$ & $\mathrm{L}$ & $\mathrm{L}$ & NA & $\mathrm{L}$ & $\mathrm{L}$ & $\mathrm{L}$ & $\mathrm{L}$ & $\mathrm{L}$ & $\mathrm{L}$ & $\mathrm{L}$ & $\mathrm{H}$ & $\mathrm{H}$ \\
\hline
\end{tabular}




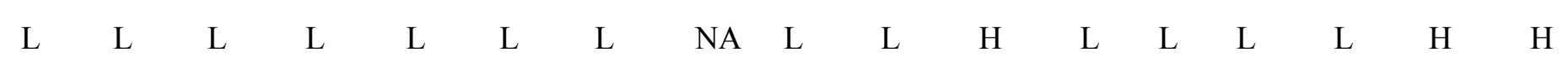

Liddle et al., 2012

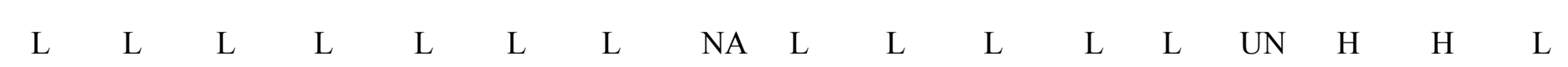

Lyngdoh et al., 2013

L L

Miura et al., 2001

Pereira et al., 2013

$$
\text { L L L }
$$

L L

L

L

NA L $\quad$ L $\quad H$

L

$\begin{array}{lllll}\text { L } & \mathrm{L} & \mathrm{L} & \mathrm{L} & \mathrm{H}\end{array}$

Porkka et al., 1994 L L L

$\mathrm{L}$

L

L NA L $\quad$ L $\quad$ H

$\begin{array}{lllllll}\text { L } & \text { UN } & \text { L } & \text { L } & \text { H } & \text { UN }\end{array}$

Porkka et al., 1994

Schmidt et al., 2011

L L L

L

L

L NA L

L L

L

Skidmore et al., 2007

Weitz et al., 2014

L L L

L

L

L L L

L

L

L $\quad$ NA $\quad$ L $\quad H \quad H$

$\begin{array}{llllll}\text { L } & \mathrm{H} & \mathrm{L} & \mathrm{L} & \mathrm{H} & \mathrm{H}\end{array}$

Wright et al., 2001

$\begin{array}{lllllllllllllllllllllllll}\text { L } & \text { L } & \text { L } & \text { L } & \text { L } & \text { L } & \text { L } & \text { NA } & \text { L } & \text { L } & \text { H } & \text { L } & \text { L } & \text { L } & \text { L } & \text { H } & \text { H }\end{array}$

\begin{tabular}{|c|c|c|c|c|c|c|c|c|c|c|c|c|c|c|c|c|c|}
\hline Wright et al., 2001 & $\mathrm{~L}$ & $\mathrm{~L}$ & $\mathrm{~L}$ & $\mathrm{~L}$ & $\mathrm{~L}$ & $\mathrm{~L}$ & $\mathrm{~L}$ & NA & $\mathrm{H}$ & $\mathrm{H}$ & $\mathrm{H}$ & $\mathrm{L}$ & $\mathrm{L}$ & $\mathrm{L}$ & $\mathrm{L}$ & $\mathrm{H}$ & $\mathrm{H}$ \\
\hline STROBE items & $\begin{array}{l}12 \\
\text { (e) }\end{array}$ & $\begin{array}{l}13 \\
\text { (a) }\end{array}$ & $\begin{array}{l}13 \\
\text { (b) }\end{array}$ & $\begin{array}{l}13 \\
\text { ('c) }\end{array}$ & $\begin{array}{l}14 \\
\text { (a) }\end{array}$ & $\begin{array}{l}14 \\
\text { (b) }\end{array}$ & $\begin{array}{l}14 \\
\text { ('c) }\end{array}$ & 15 & $\begin{array}{l}16 \\
\text { (a) }\end{array}$ & $\begin{array}{l}16 \\
\text { (b) }\end{array}$ & $\begin{array}{l}16 \\
\text { ('c) }\end{array}$ & 17 & 18 & 19 & 20 & 21 & 22 \\
\hline
\end{tabular}

$\begin{array}{llllllllllllllllllllllllllllllllll}\text { Abraham et al., } 1971 & \text { H } & \text { L } & \text { H } & \text { H } & \text { H } & \text { L } & \text { L } & \text { L } & \text { L } & \text { L } & \text { H } & \text { H } & \text { L } & \text { H } & \text { L } & \text { UN } & \text { H }\end{array}$

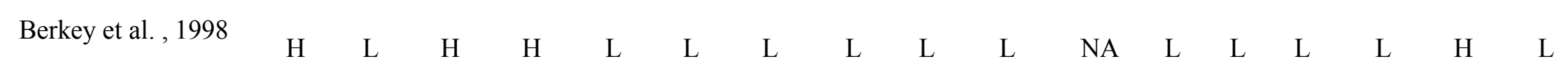

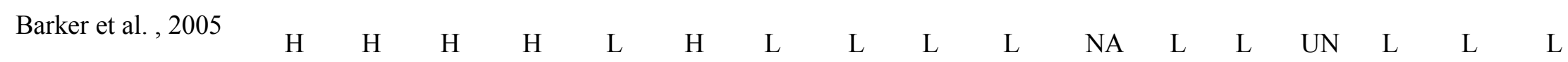

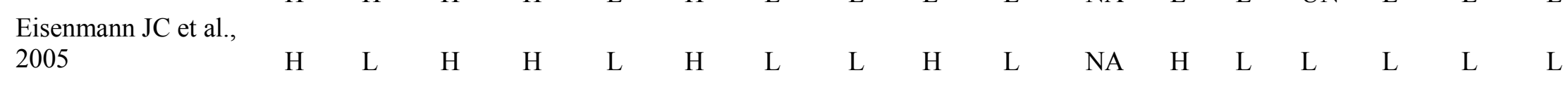


Freedman DS et al.,

Gustafsson et al.,

2011

$\begin{array}{cccccccccccccccccc}\text { H } & \text { L } & \text { UN } & \text { H } & \text { L } & \text { H } & \text { L } & \text { L } & \text { L } & \text { L } & \text { NA } & \text { L } & \text { L } & \text { L } & \text { L } & \text { L } & \text { L } \\ \text { H } & \text { L } & \text { L } & \text { H } & \text { L } & \text { L } & \text { L } & \text { L } & \text { L } & \text { L } & \text { NA } & \text { L } & \text { L } & \text { L } & \text { L } & \text { L } & \text { H } \\ \text { L } & \text { L } & \text { L } & \text { L } & \text { L } & \text { L } & \text { L } & \text { L } & \text { L } & \text { L } & \text { H } & \text { L } & \text { L } & \text { L } & \text { L } & \text { L } & \text { L } \\ \text { H } & \text { H } & \text { H } & \text { H } & \text { H } & \text { H } & \text { H } & \text { H } & \text { L } & \text { L } & \text { L } & \text { L } & \text { L } & \text { L } & \text { L } & \text { L } & \text { H } \\ \text { H } & \text { H } & \text { H } & \text { H } & \text { L } & \text { L } & \text { H } & \text { H } & \text { H } & \text { L } & \text { H } & \text { L } & \text { L } & \text { H } & \text { L } & \text { L } & \text { L } \\ \text { H } & \text { H } & \text { H } & \text { H } & \text { H } & \text { L } & \text { L } & \text { L } & \text { L } & \text { L } & \text { NA } & \text { L } & \text { L } & \text { H } & \text { L }\end{array}$

2000

Kneisley et al., 1990

Koziel et al., 2011

Lauer et al., 1993

Li et al., 2007

Liddle et al., 2012

Lyngdoh et al., 2013

Miura et al., 2001

Pereira et al., 2013

Porkka et al., 1994

$\begin{array}{lllll}\mathrm{H} & \mathrm{H} & \mathrm{H} & \mathrm{H} & \mathrm{H}\end{array}$

$\mathrm{H}$

$\begin{array}{llll}\mathrm{H} & \mathrm{H} & \mathrm{H} & \mathrm{H}\end{array}$

$\mathrm{H} \quad \mathrm{H}$

H

$\mathrm{H} \quad \mathrm{H}$

L

NA

L

$\begin{array}{llll}\mathrm{L} & \mathrm{H} & \mathrm{H} & \mathrm{H}\end{array}$

L $\quad$ L $\quad \mathrm{H} \quad \mathrm{H}$

$\mathrm{H} \quad \mathrm{H}$

$\mathrm{H} \quad \mathrm{H} \quad \mathrm{H} \quad \mathrm{H}$

L L

$\mathrm{H}$

L

$\mathrm{H}$

NA

NA

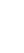

$\mathrm{H}$

$\begin{array}{llll}\mathrm{L} & \mathrm{H} & \mathrm{H} & \mathrm{H}\end{array}$

$\begin{array}{llllll}\text { L } & \mathrm{L} & \mathrm{H} & \mathrm{H} & \mathrm{H}\end{array}$

$\begin{array}{lllllll}\mathrm{H} & \mathrm{L} & \mathrm{H} & \mathrm{H} & \mathrm{H} & \mathrm{L}\end{array}$

$\mathrm{H}$

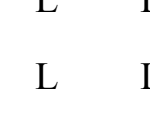

$\mathrm{L}$

L




$\begin{array}{lccccccccccccccccc}\text { Schmidt et al., 2011 } & \text { H } & \text { L } & \text { H } & \text { H } & \text { H } & \text { L } & \text { L } & \text { L } & \text { L } & \text { L } & \text { H } & \text { L } & \text { L } & \text { L } & \text { L } & \text { L } & \text { L } \\ \text { Skidmore et al., 2007 } & \text { L } & \text { H } & \text { L } & \text { H } & \text { H } & \text { L } & \text { L } & \text { L } & \text { L } & \text { NA } & \text { NA } & \text { L } & \text { L } & \text { L } & \text { L } & \text { L } & \text { L } \\ \text { Weitz et al., 2014 } & \text { H } & \text { L } & \text { L } & \text { H } & \text { H } & \text { L } & \text { L } & \text { L } & \text { L } & \text { NA } & \text { NA } & \text { L } & \text { L } & \text { L } & \text { L } & \text { L } & \text { H } \\ \text { Wright et al., 2001 } & \text { H } & \text { H } & \text { L } & \text { H } & \text { H } & \text { H } & \text { H } & \text { H } & \text { L } & \text { NA } & \text { NA } & \text { L } & \text { L } & \text { L } & \text { L } & \text { L } & \text { L }\end{array}$

NA-Not Applicable

L- Low Risk

H- High Risk

UN- Unclear

\section{STROBE Statement—checklist of items that should be included in reports of observational studies}

1 a. Indicate the study's design with a commonly used term in the title or the abstract

$1 \mathrm{~b}$. Provide in the abstract an informative and balanced summary of what was done and what was found

2. Explain the scientific background and rationale for the investigation being reported

3. State specific objectives, including any pre-specified hypotheses

4. Present key elements of study design early in the paper

5. Describe the setting, locations, and relevant dates, including periods of recruitment, exposure, follow-up, and data collection

6 a. Give the eligibility criteria, and the sources and methods of selection of participants. Describe methods of follow-up

$6 \mathrm{~b}$. For matched studies, give matching criteria and number of exposed and unexposed-Not Applicable for any study therefore this item is not shown in the figure below

7. Clearly define all outcomes, exposures, predictors, potential confounders, and effect modifiers. Give diagnostic criteria, if applicable

8. For each variable of interest, give sources of data and details of methods of assessment. Describe comparability of assessment methods if there

is more than one group

9. Describe any efforts to address potential sources of bias

10. Explain how the study size was arrived at.

11. Explain how quantitative variables were handled in the analyses. If applicable, describe which groupings were chosen and why

12 a. Describe all statistical methods, including those used to control for confounding

$12 \mathrm{~b}$. Describe any methods used to examine subgroups and interactions 
$12 \mathrm{c}$. Explain how missing data were addressed

12 d. Cohort study - If applicable, explain how loss to follow-up was addressed

$12 \mathrm{e}$. Describe any sensitivity analyses

13 a. Report numbers of individuals at each stage of study

$13 \mathrm{~b}$. Give reasons for non-participation at each stage

$13 \mathrm{c}$. Consider use of a flow diagram

14 a. Give characteristics of study participants

$14 \mathrm{~b}$. Indicate numbers of participants with missing data for each variable of interest

$14 \mathrm{c}$. Summaries follow-up time

15. Report numbers of outcome events or summary measures over time

16 a. Give unadjusted estimates and, if applicable, confounder-adjusted estimates and their precision. Make clear which confounders were adjusted for and why they were included

$16 \mathrm{~b}$. Report category boundaries when continuous variables were categorized

$16 \mathrm{c}$. If relevant, consider translating estimates of relative risk into absolute risk for a meaningful time period

17. Report other analyses done — eg analyses of subgroups and interactions, and sensitivity analyses

18. Summaries key results with reference to study objectives

19. Discuss limitations of the study, taking into account sources of potential bias or imprecision. Discuss both direction and magnitude of any potential bias

20. Give a cautious overall interpretation of results considering objectives, limitations, and multiplicity of analyses, results from similar studies, and other relevant evidence

21. Discuss the generalizability (external validity) of the study results

22. Give the source of funding and the role of the funders for the present study and, if applicable, for the original study on which the present article is based

\section{Risk of Bias Assessment:}

Figure: Risk of bias assessment for each item of the STROBE instrument 


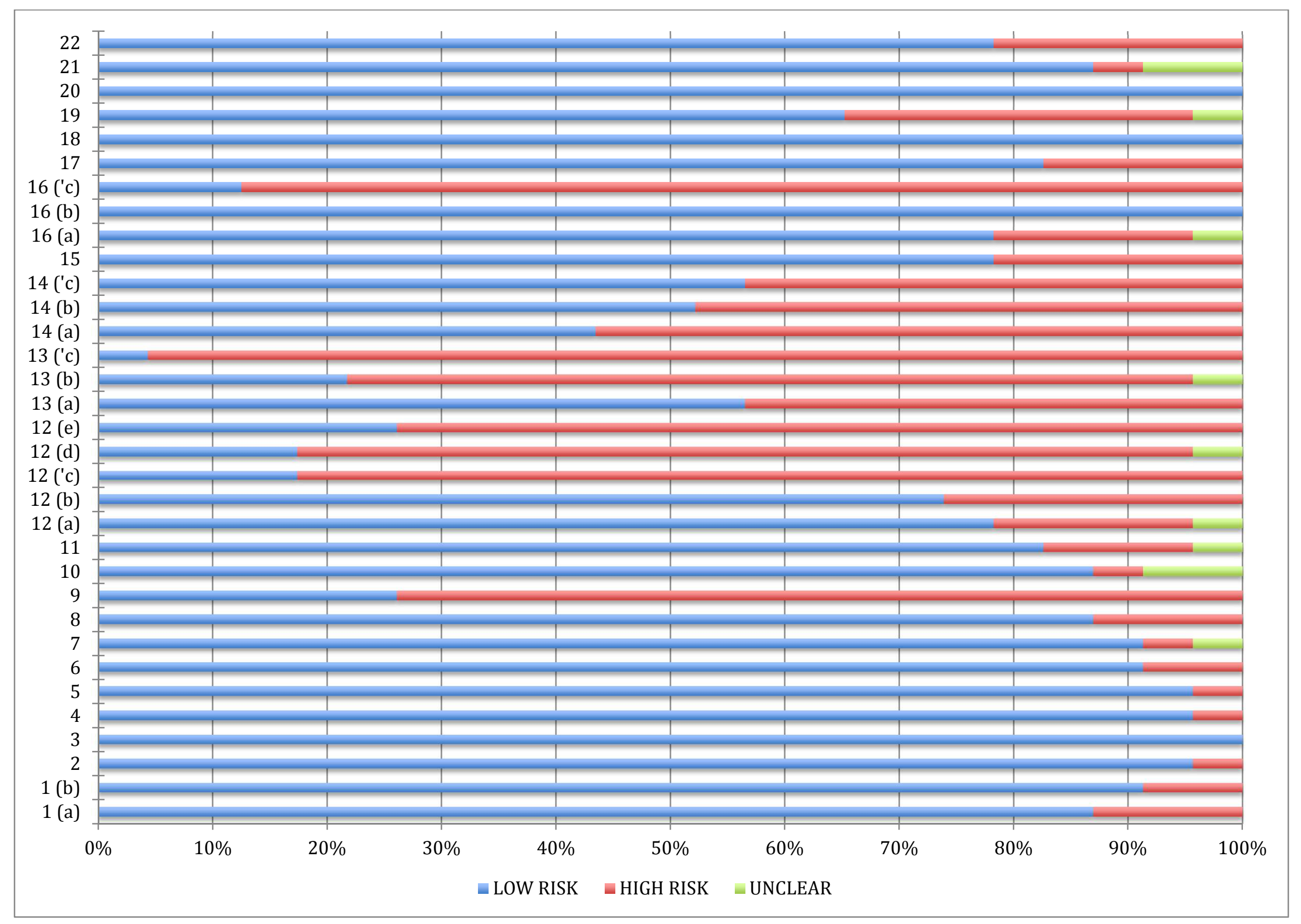


Additional File 4:

Influence meta-analysis, Cumulative meta-analysis and Funnel plots

\section{Influence Analysis}

Figure 1a. Influence analysis for the association between childhood obesity and adult SBP

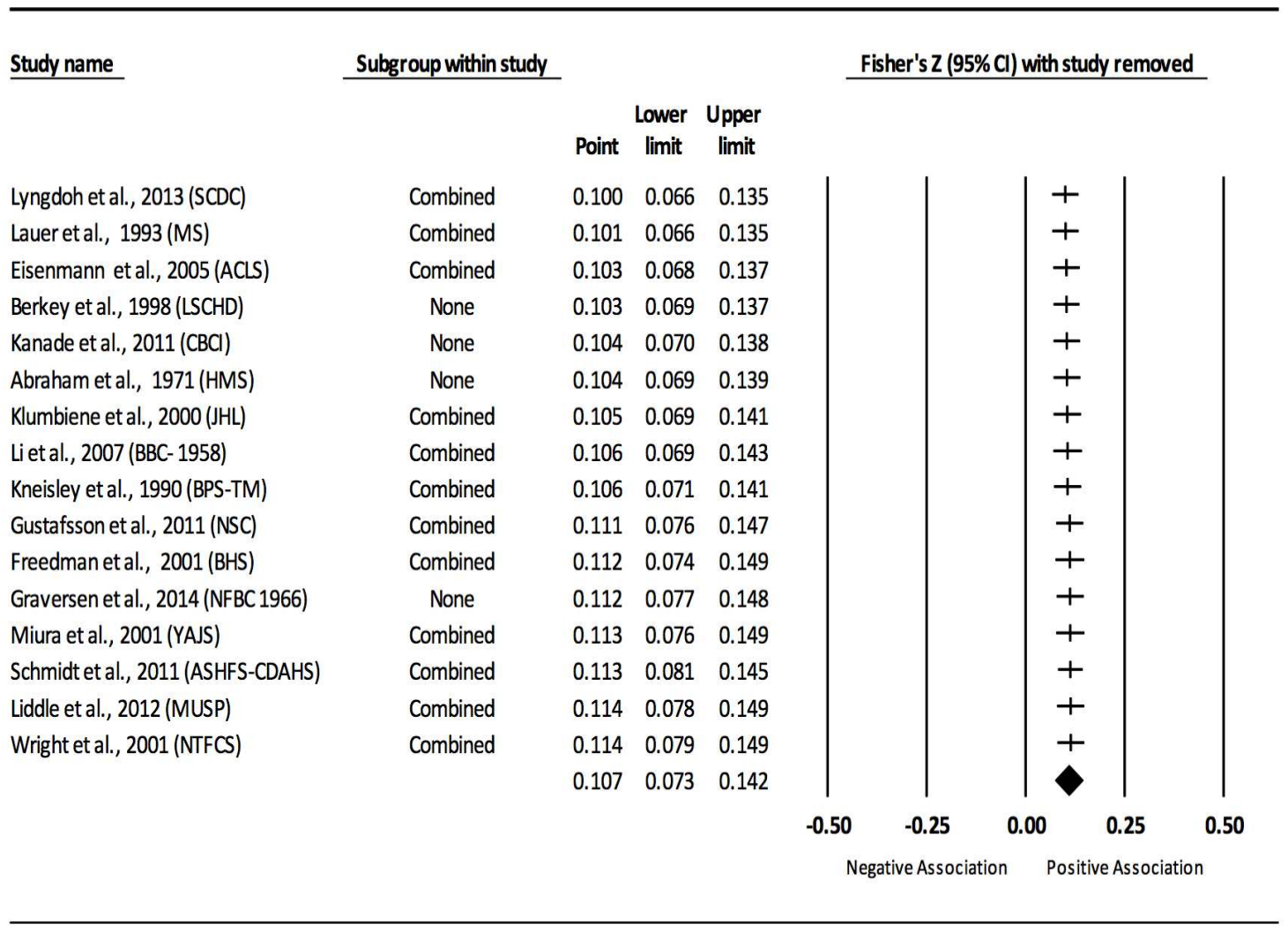

Influence analysis for point estimate changes in SBP with each corresponding study deleted from the model once. The vertical lines represent the represent the Fishers Zr while the left and right extremes of the squares represent the corresponding $95 \%$ confidence intervals. The middle of the black diamond represents the overall mean difference while the left and right extremes of the diamond represent the corresponding 95\% confidence intervals. Results are ordered from smallest to largest reductions. Combined measures represent those studies in which male and female were combined, or different age cohorts from each study were combines, or multiple readings from the same cohort were combined or one study using more than one exposure definition. 
Figure 1b. Influence analysis for the association between childhood obesity and adult SBP adjusted for adult BMI

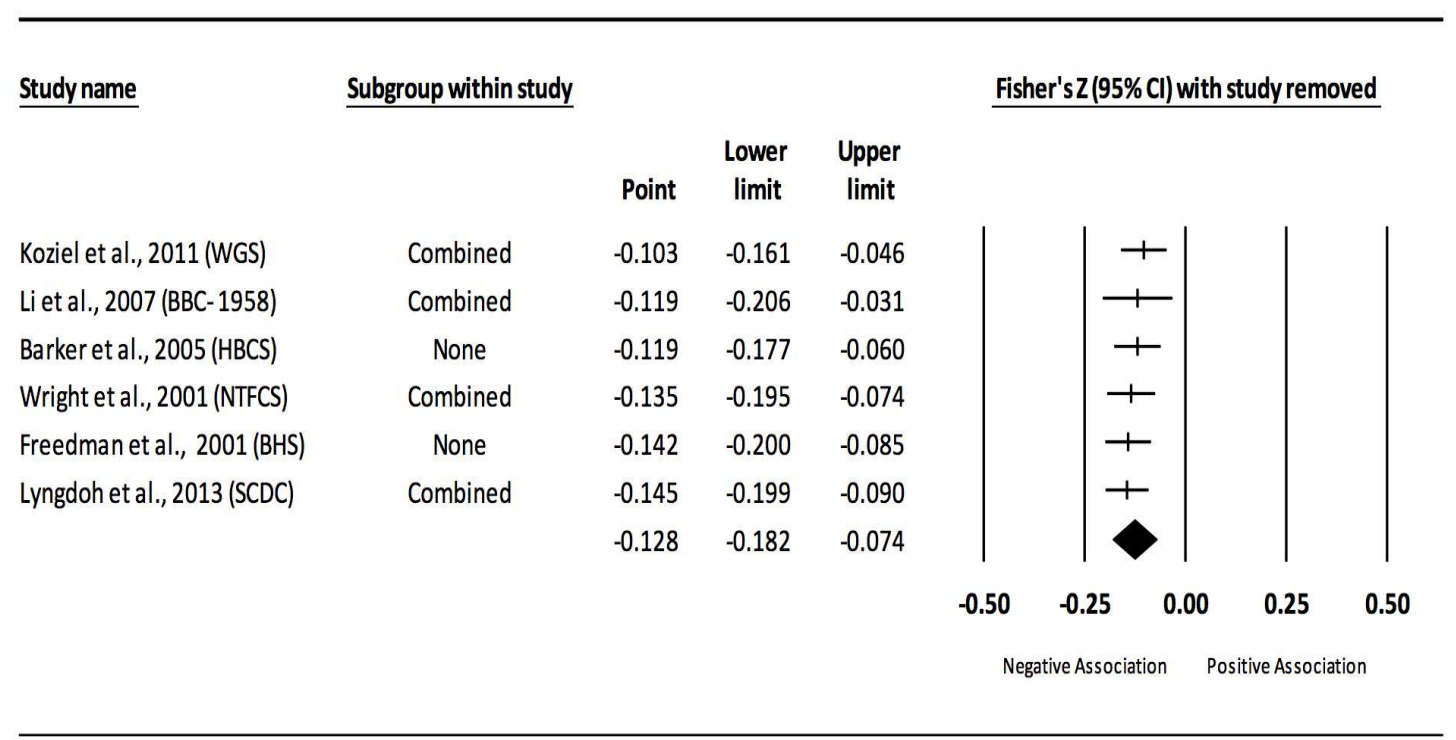

Influence analysis for point estimate changes in SBP with each corresponding study deleted from the model once. The vertical lines represent the represent the Fishers $\mathrm{Zr}$ while the left and right extremes of the squares represent the corresponding $95 \%$ confidence intervals. The middle of the black diamond represents the overall mean difference while the left and right extremes of the diamond represent the corresponding 95\% confidence intervals. Results are ordered from smallest to largest reductions. Combined measures represent those studies in which male and female were combined, or different age cohorts from each study were combines, or multiple readings from the same cohort were combined or one study using more than one exposure definition. 
Figure 2a. Influence analysis for the association between childhood obesity and adult DBP

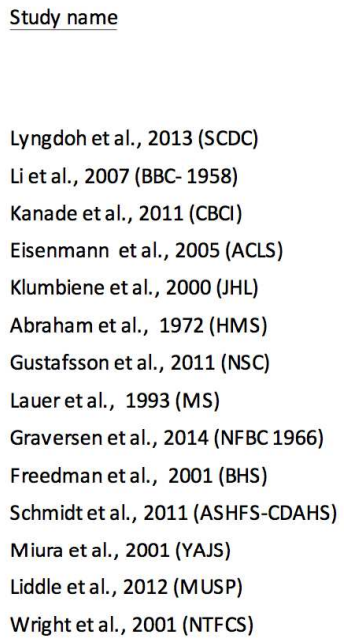

Subgroup within study

Combined

Combined

Combined

Combined

Combined

None

Combined

Combined

None

Combined

Combined

Combined

Combined

Combined
Fisher's Z $(95 \% \mathrm{Cl})$ with study removed

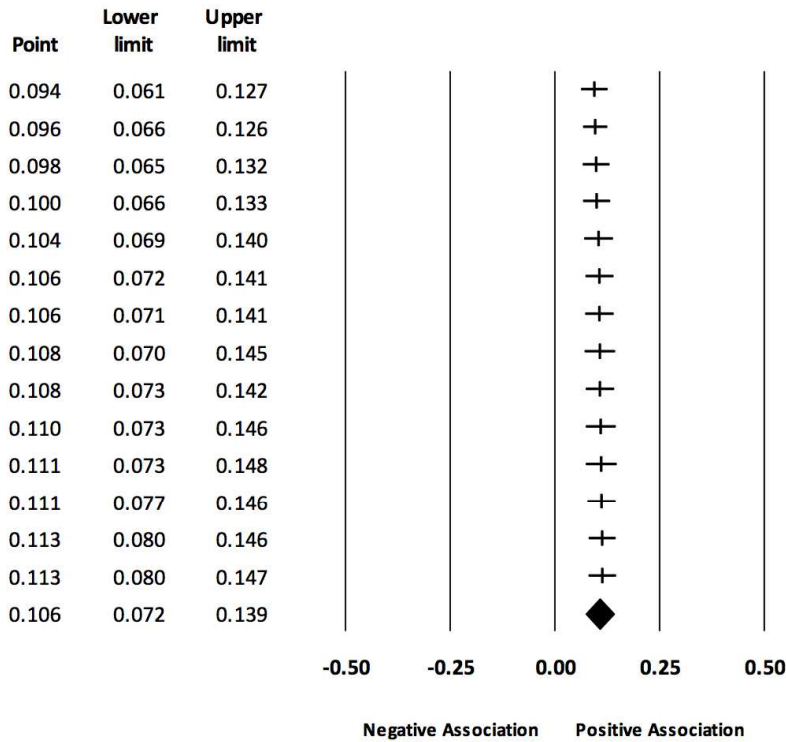

Influence analysis for point estimate changes in DBP with each corresponding study deleted from the model once. The vertical lines represent the represent the Fishers $\mathrm{Zr}$ while the left and right extremes of the squares represent the corresponding $95 \%$ confidence intervals. The middle of the black diamond represents the overall mean difference while the left and right extremes of the diamond represent the corresponding 95\% confidence intervals. Results are ordered from smallest to largest reductions. Combined measures represent those studies in which male and female were combined, or different age cohorts from each study were combines, or multiple readings from the same cohort were combined or one study using more than one exposure definition. 
Figure 2b. Influence analysis for the association between childhood obesity and adult DBP adjusted for adult BMI

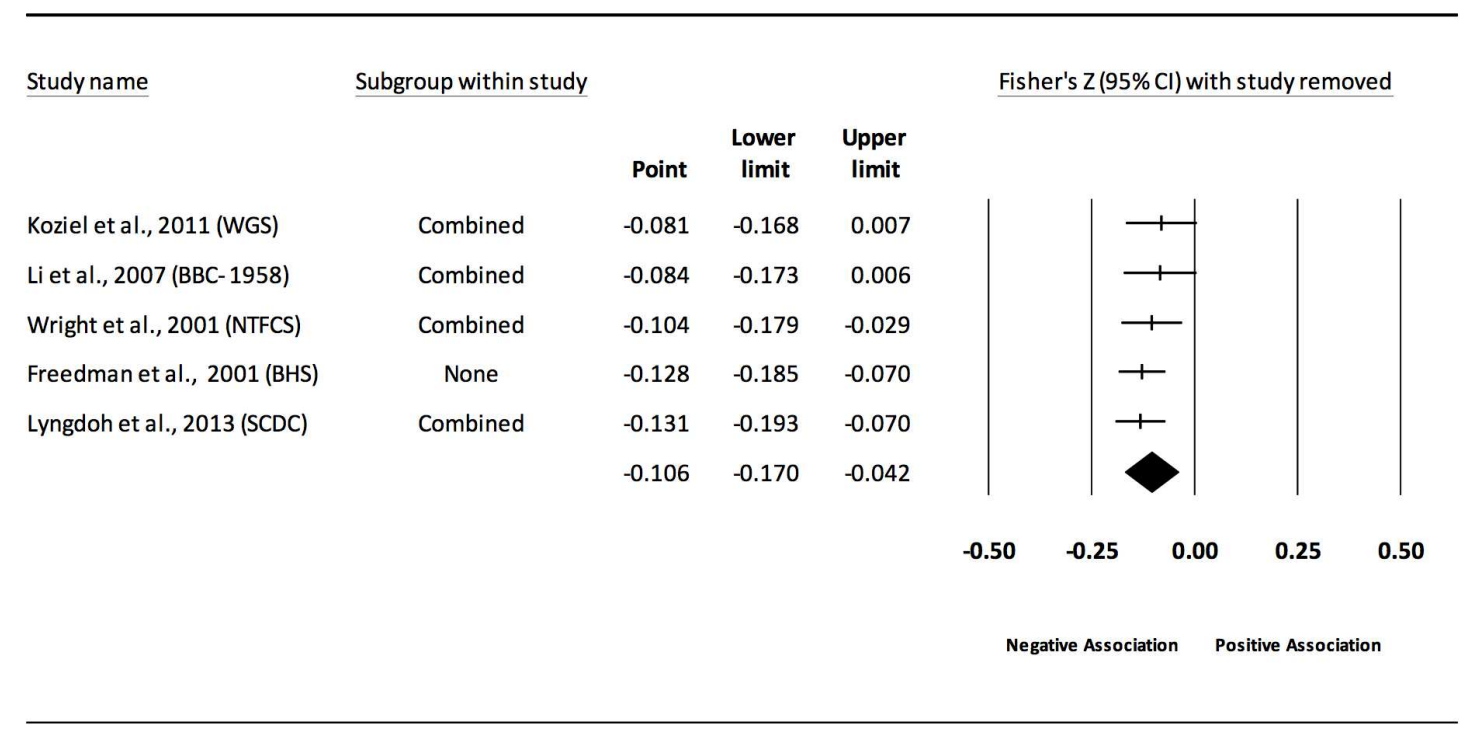

Influence analysis for point estimate changes in DBP with each corresponding study deleted from the model once. The vertical lines represent the represent the Fishers $\mathrm{Zr}$ while the left and right extremes of the squares represent the corresponding $95 \%$ confidence intervals. The middle of the black diamond represents the overall mean difference while the left and right extremes of the diamond represent the corresponding 95\% confidence intervals. Results are ordered from smallest to largest reductions. Combined measures represent those studies in which male and female were combined, or different age cohorts from each study were combines, or multiple readings from the same cohort were combined or one study using more than one exposure definition. 
Figure 3a. Influence analysis for the association between childhood obesity and adult TC

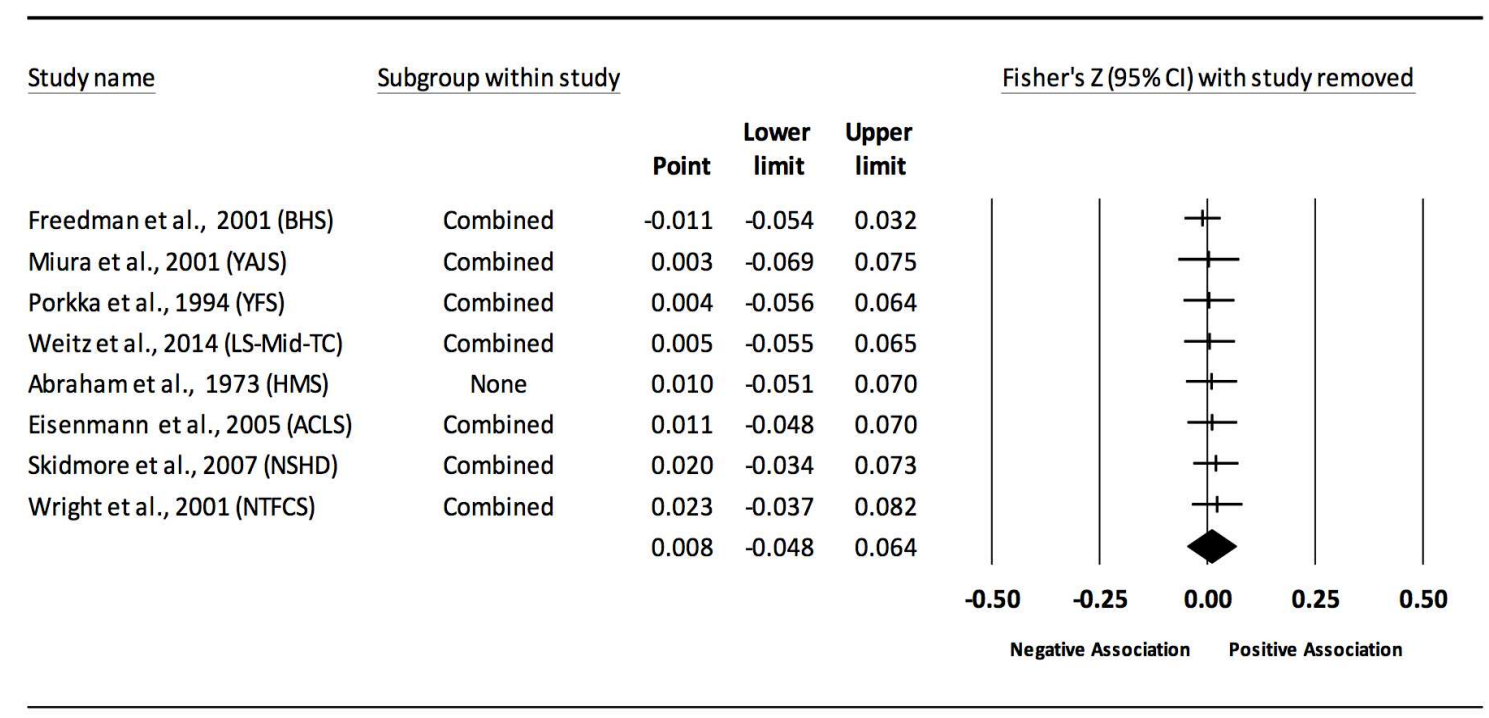

Influence analysis for point estimate changes in TC with each corresponding study deleted from the model once. The vertical lines represent the represent the Fishers $\mathrm{Zr}$ while the left and right extremes of the squares represent the corresponding $95 \%$ confidence intervals. The middle of the black diamond represents the overall mean difference while the left and right extremes of the diamond represent the corresponding 95\% confidence intervals. Results are ordered from smallest to largest reductions. Combined measures represent those studies in which male and female were combined, or different age cohorts from each study were combines, or multiple readings from the same cohort were combined or one study using more than one exposure definition. 
Figure 3b. Influence analysis for the association between childhood obesity and adult TC adjusted for adult BMI

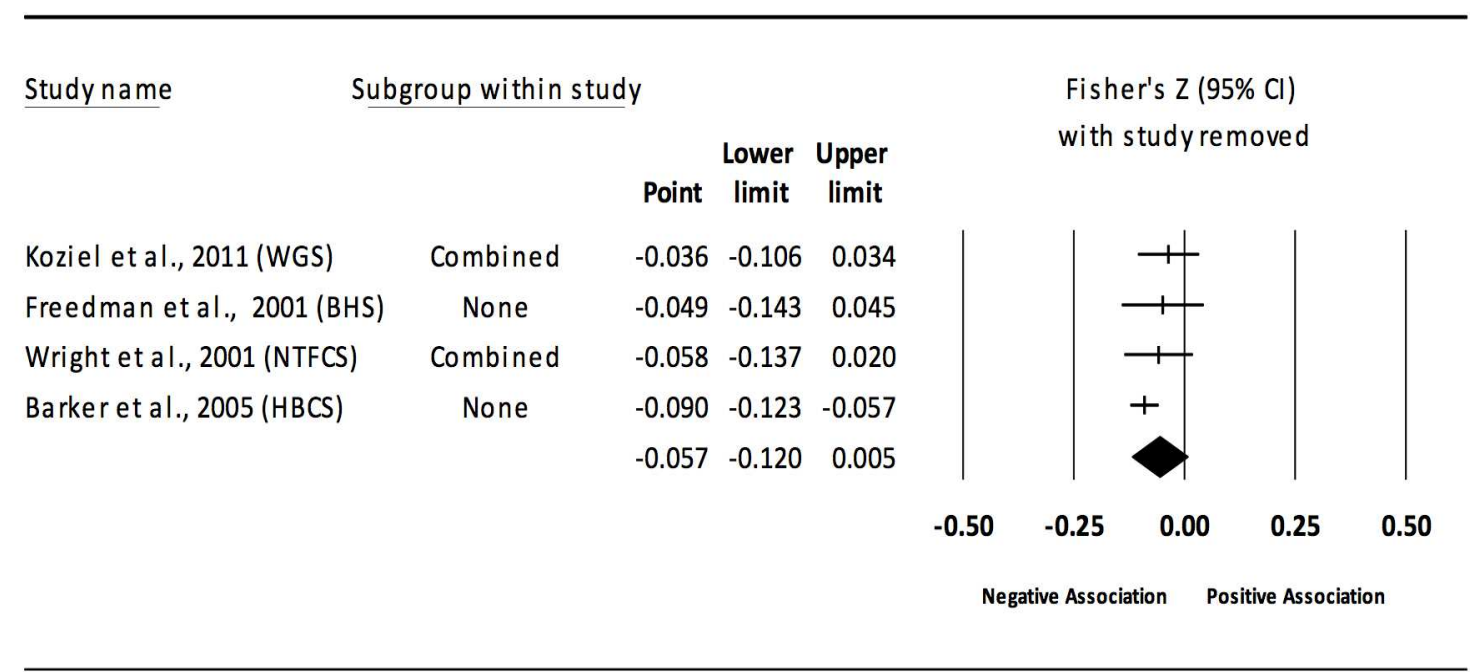

Influence analysis for point estimate changes in TC with each corresponding study deleted from the model once. The vertical lines represent the represent the Fishers $\mathrm{Zr}$ while the left and right extremes of the squares represent the corresponding $95 \%$ confidence intervals. The middle of the black diamond represents the overall mean difference while the left and right extremes of the diamond represent the corresponding 95\% confidence intervals. Results are ordered from smallest to largest reductions. Combined measures represent those studies in which male and female were combined, or different age cohorts from each study were combines, or multiple readings from the same cohort were combined or one study using more than one exposure definition. 
Figure 4a. Influence analysis for the association between childhood obesity and adult LDL

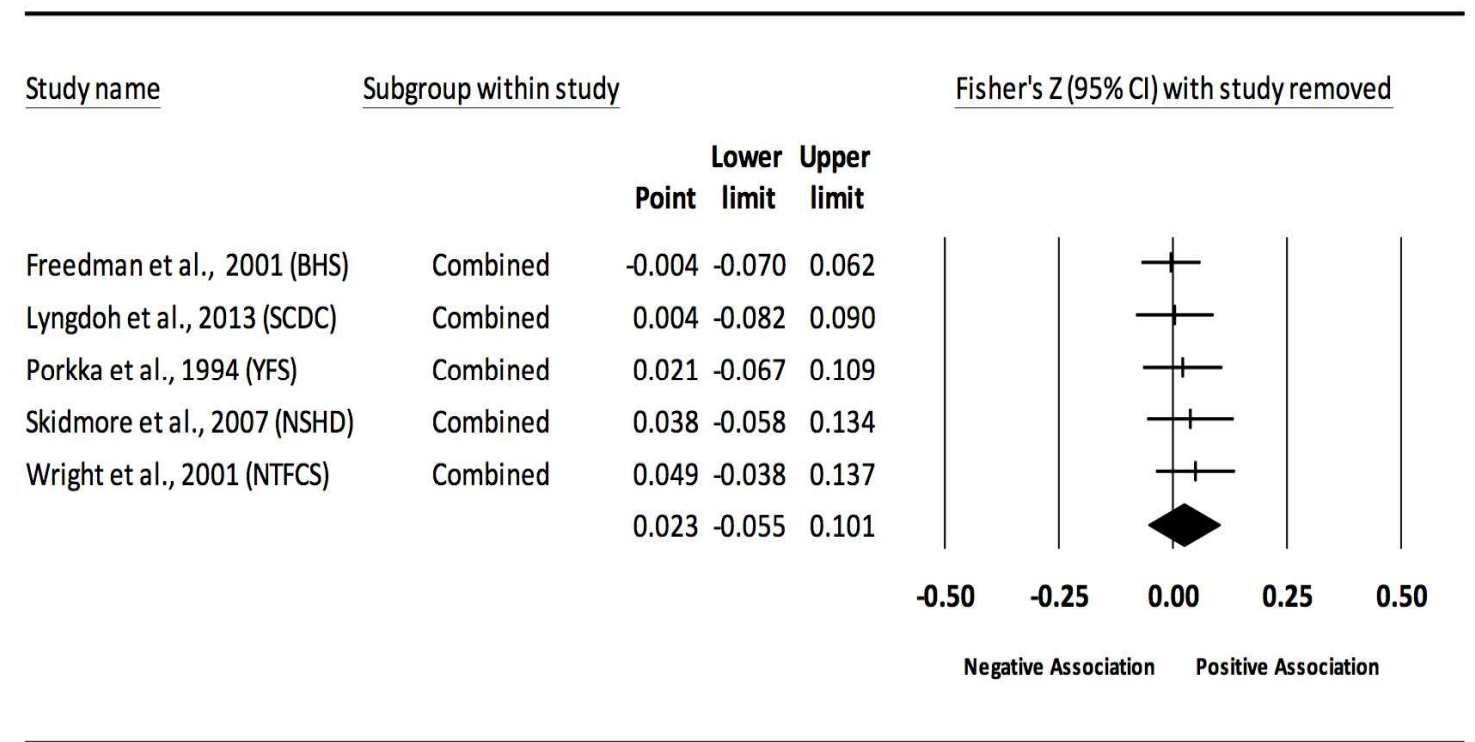

Influence analysis for point estimate changes in LDL with each corresponding study deleted from the model once. The vertical lines represent the represent the Fishers Zr while the left and right extremes of the squares represent the corresponding $95 \%$ confidence intervals. The middle of the black diamond represents the overall mean difference while the left and right extremes of the diamond represent the corresponding 95\% confidence intervals. Results are ordered from smallest to largest reductions. Combined measures represent those studies in which male and female were combined, or different age cohorts from each study were combines, or multiple readings from the same cohort were combined or one study using more than one exposure definition. 
Figure $4 \mathrm{~b}$. Influence analysis for the association between childhood obesity and adult LDL adjusted for adult BMI

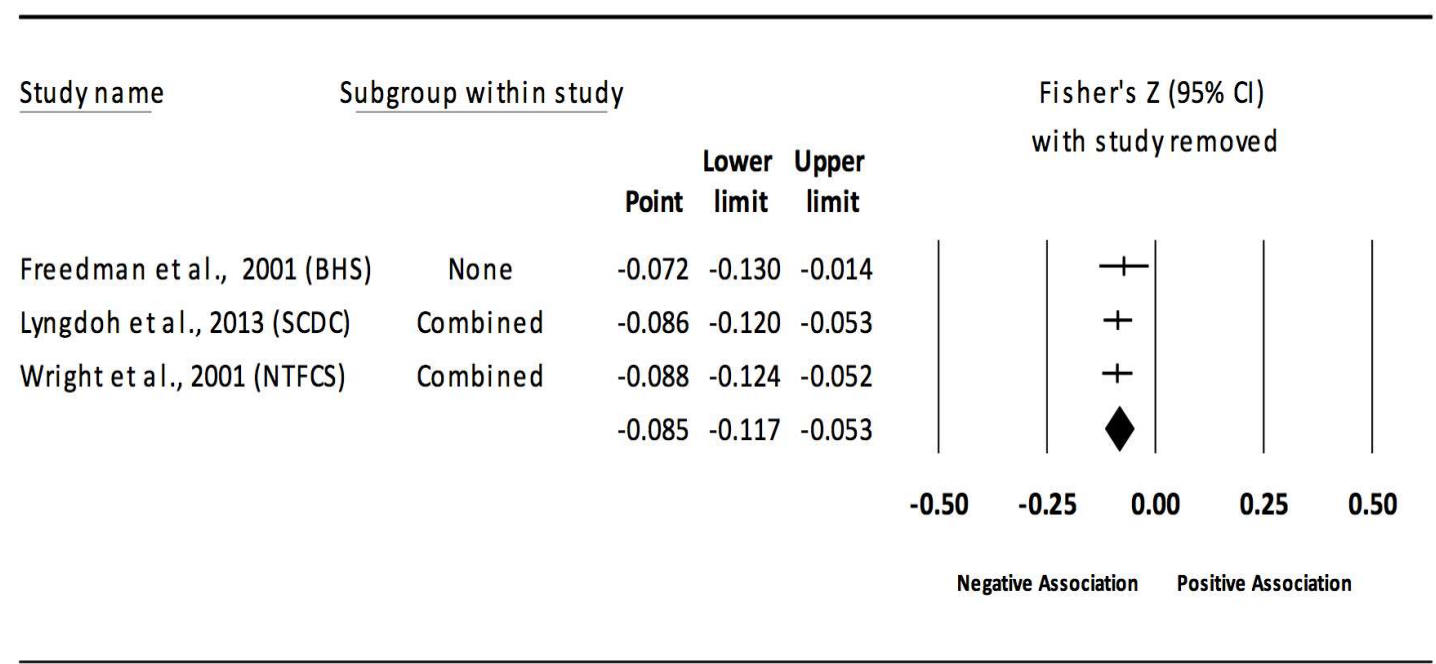

Influence analysis for point estimate changes in LDL with each corresponding study deleted from the model once. The vertical lines represent the represent the Fishers $\mathrm{Zr}$ while the left and right extremes of the squares represent the corresponding $95 \%$ confidence intervals. The middle of the black diamond represents the overall mean difference while the left and right extremes of the diamond represent the corresponding 95\% confidence intervals. Results are ordered from smallest to largest reductions. Combined measures represent those studies in which male and female were combined, or different age cohorts from each study were combines, or multiple readings from the same cohort were combined or one study using more than one exposure definition. 
Figure 5a. Influence analysis for the association between childhood obesity and adult HDL

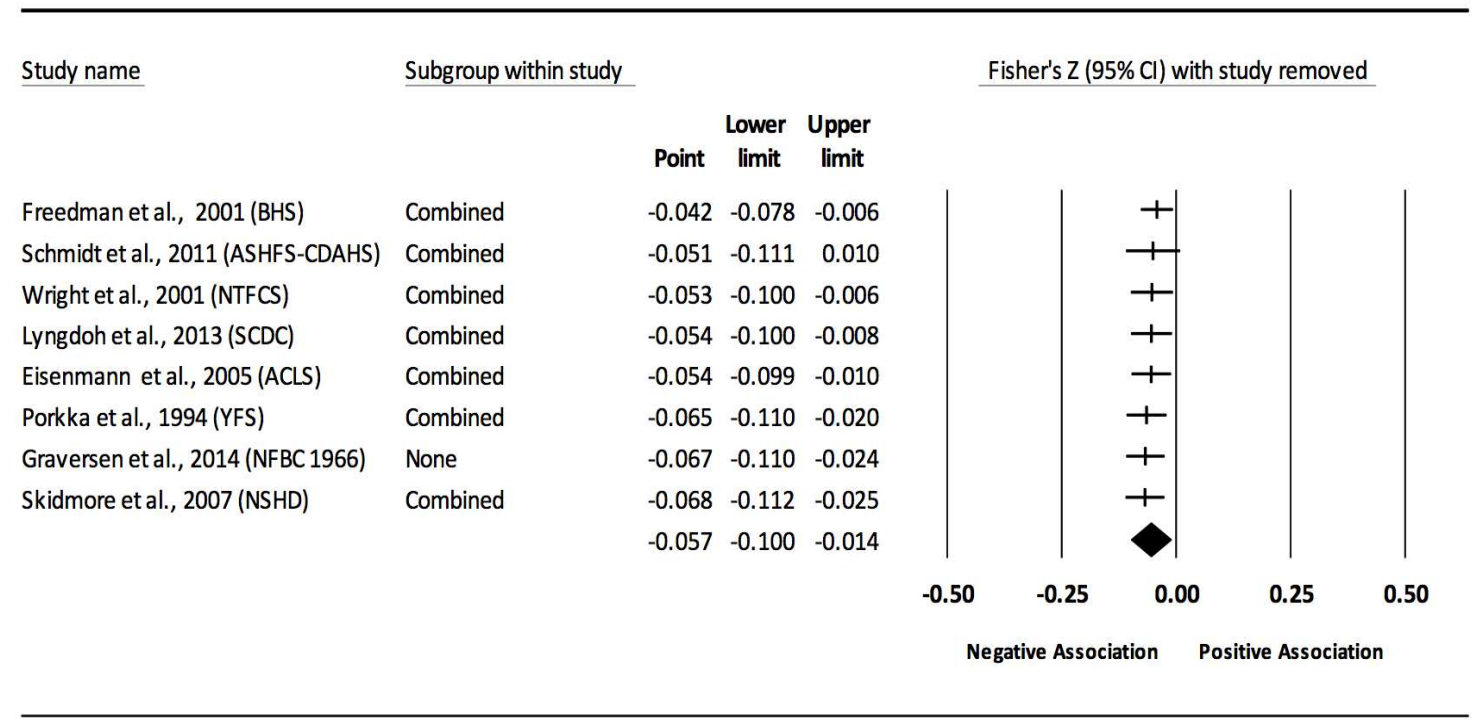

Influence analysis for point estimate changes in HDL with each corresponding study deleted from the model once. The vertical lines represent the represent the Fishers $\mathrm{Zr}$ while the left and right extremes of the squares represent the corresponding $95 \%$ confidence intervals. The middle of the black diamond represents the overall mean difference while the left and right extremes of the diamond represent the corresponding 95\% confidence intervals. Results are ordered from smallest to largest reductions. Combined measures represent those studies in which male and female were combined, or different age cohorts from each study were combines, or multiple readings from the same cohort were combined or one study using more than one exposure definition. 
Figure 5b. Influence analysis for the association between childhood obesity and adult HDL adjusted for adult BMI

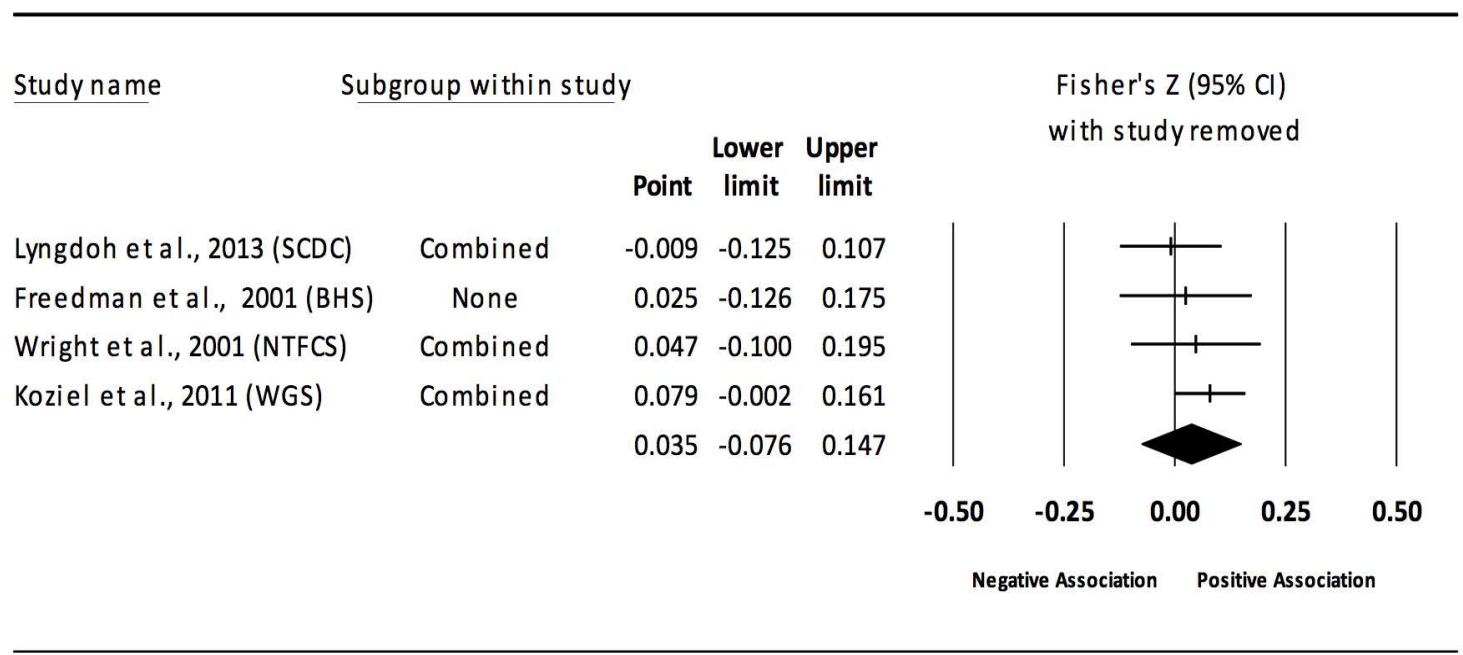

Influence analysis for point estimate changes in HDL with each corresponding study deleted from the model once. The vertical lines represent the represent the Fishers $\mathrm{Zr}$ while the left and right extremes of the squares represent the corresponding $95 \%$ confidence intervals. The middle of the black diamond represents the overall mean difference while the left and right extremes of the diamond represent the corresponding 95\% confidence intervals. Results are ordered from smallest to largest reductions. Combined measures represent those studies in which male and female were combined, or different age cohorts from each study were combines, or multiple readings from the same cohort were combined or one study using more than one exposure definition. 
Figure 6a. Influence analysis for the association between childhood obesity and adult TG

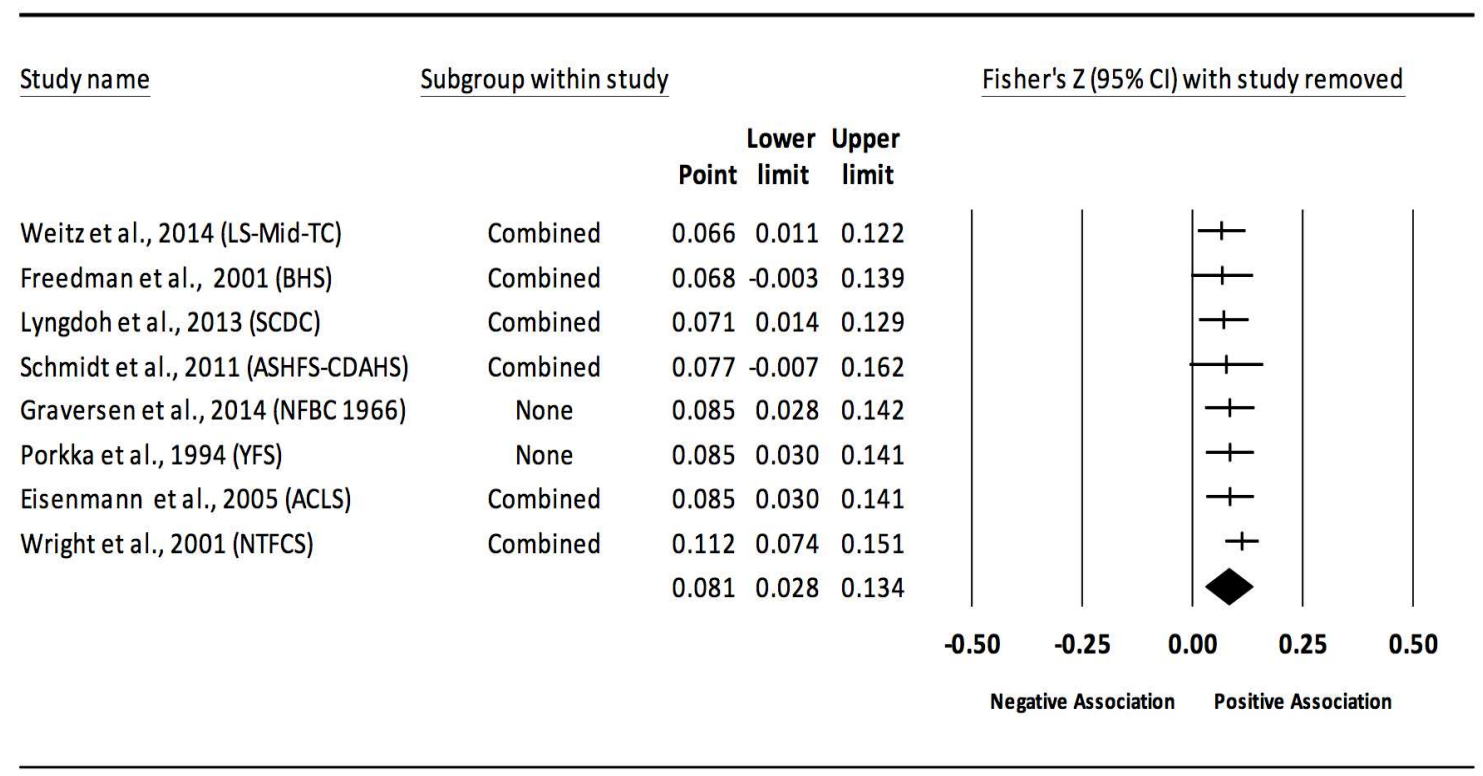

Influence analysis for point estimate changes in TG with each corresponding study deleted from the model once. The vertical lines represent the represent the Fishers $\mathrm{Zr}$ while the left and right extremes of the squares represent the corresponding $95 \%$ confidence intervals. The middle of the black diamond represents the overall mean difference while the left and right extremes of the diamond represent the corresponding 95\% confidence intervals. Results are ordered from smallest to largest reductions. Combined measures represent those studies in which male and female were combined, or different age cohorts from each study were combines, or multiple readings from the same cohort were combined or one study using more than one exposure definition. 
Figure 6b. Influence analysis for the association between childhood obesity and adult TG adjusted for adult BMI

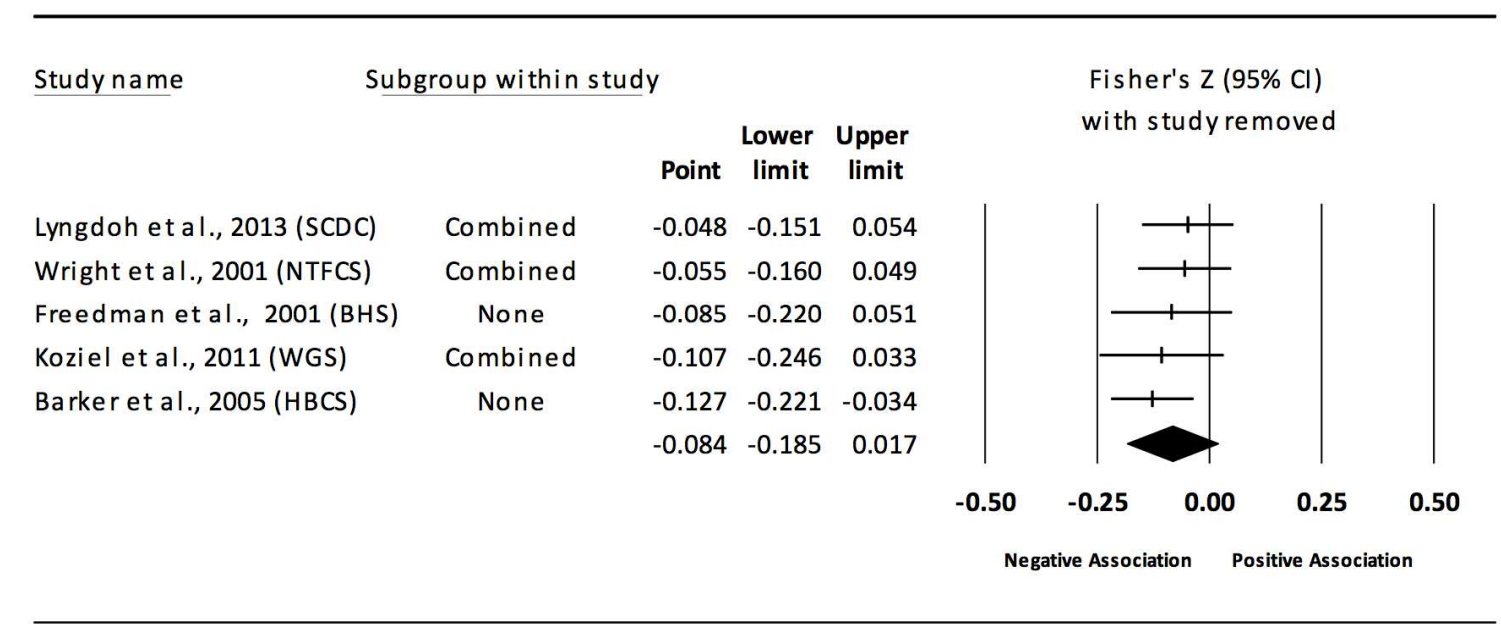

Influence analysis for point estimate changes in TG with each corresponding study deleted from the model once. The vertical lines represent the represent the Fishers $\mathrm{Zr}$ while the left and right extremes of the squares represent the corresponding $95 \%$ confidence intervals. The middle of the black diamond represents the overall mean difference while the left and right extremes of the diamond represent the corresponding 95\% confidence intervals. Results are ordered from smallest to largest reductions. Combined measures represent those studies in which male and female were combined, or different age cohorts from each study were combines, or multiple readings from the same cohort were combined or one study using more than one exposure definition. 
Cumulative Meta-Analysis:

Figure 1a. Cumulative meta-analysis for the association between childhood obesity and adult SBP

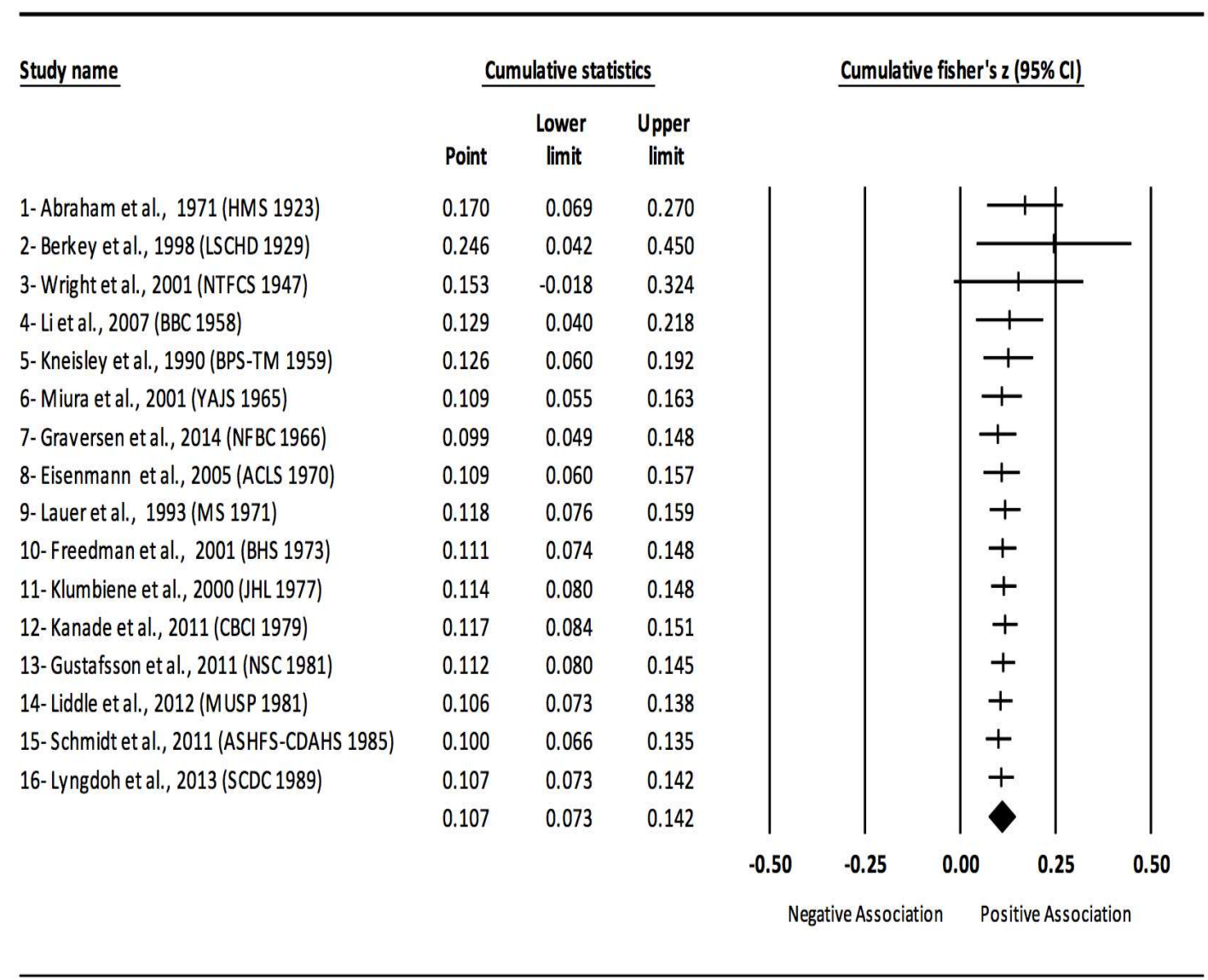

Cumulative meta-analysis, ordered by the year the study started. The vertical lines represent the represent the Fishers $\mathrm{Zr}$ while the left and right extremes of the vertical lines represent the corresponding $95 \%$ confidence intervals. The results of each corresponding study are pooled with all studies preceding it. The middle of the black diamond represents the overall Fishers Zr while the left and right extremes of the diamond represent the corresponding $95 \%$ confidence intervals. Combined measures represent those studies in which male and female were combined, or different age cohorts from each study were combines, or multiple readings from the same cohort were combined or one study using more than one exposure definition. 
Figure 1b. Cumulative meta-analysis for the association between childhood obesity and adult SBP adjusted for adult BMI

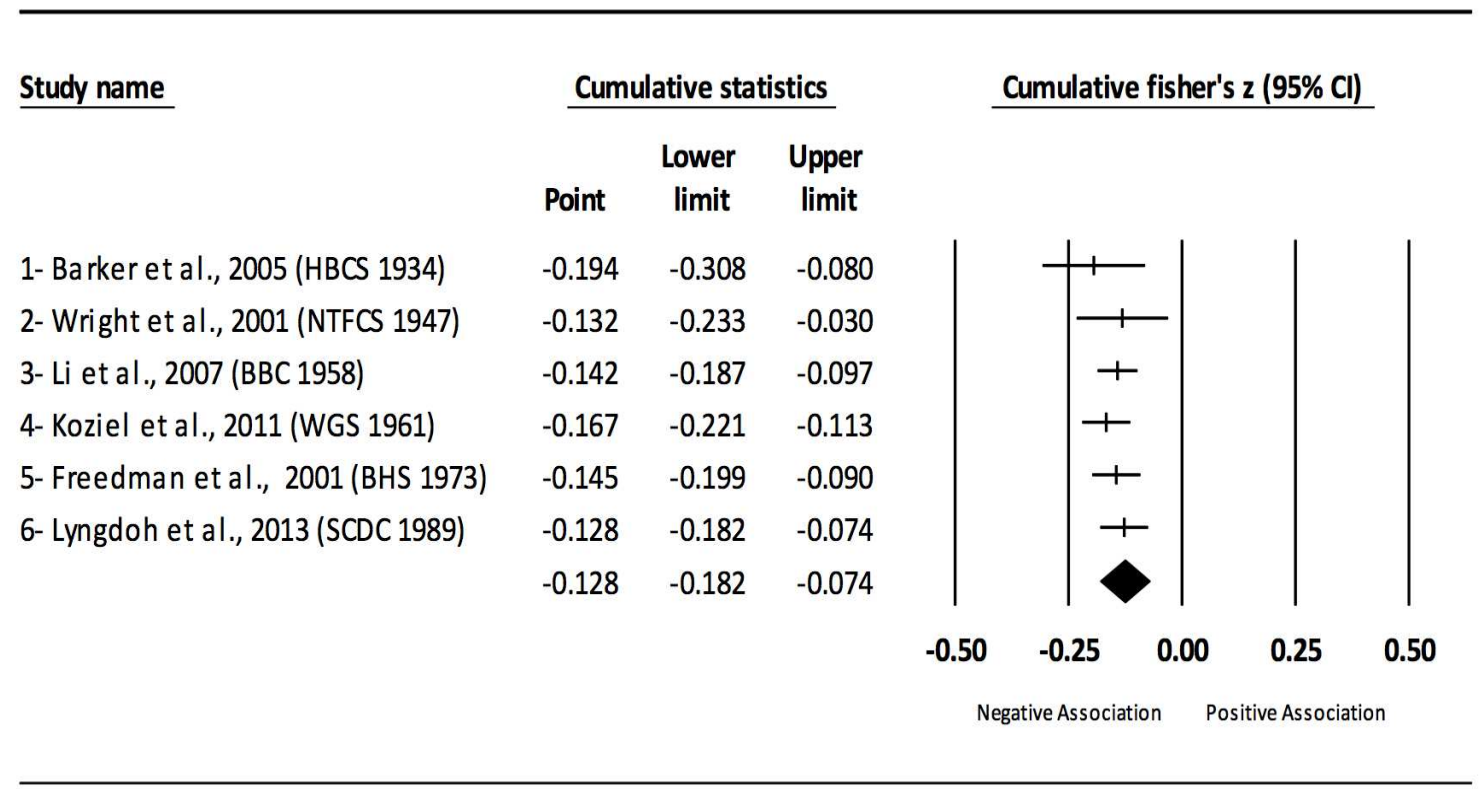

Cumulative meta-analysis, ordered by the year the study started. The vertical lines represent the represent the Fishers $\mathrm{Zr}$ while the left and right extremes of the vertical lines represent the corresponding $95 \%$ confidence intervals. The results of each corresponding study are pooled with all studies preceding it. The middle of the black diamond represents the overall Fishers $\mathrm{Zr}$ while the left and right extremes of the diamond represent the corresponding $95 \%$ confidence intervals. Combined measures represent those studies in which male and female were combined, or different age cohorts from each study were combines, or multiple readings from the same cohort were combined or one study using more than one exposure definition. 
Figure 2a. Cumulative meta-analysis for the association between childhood obesity and adult DBP

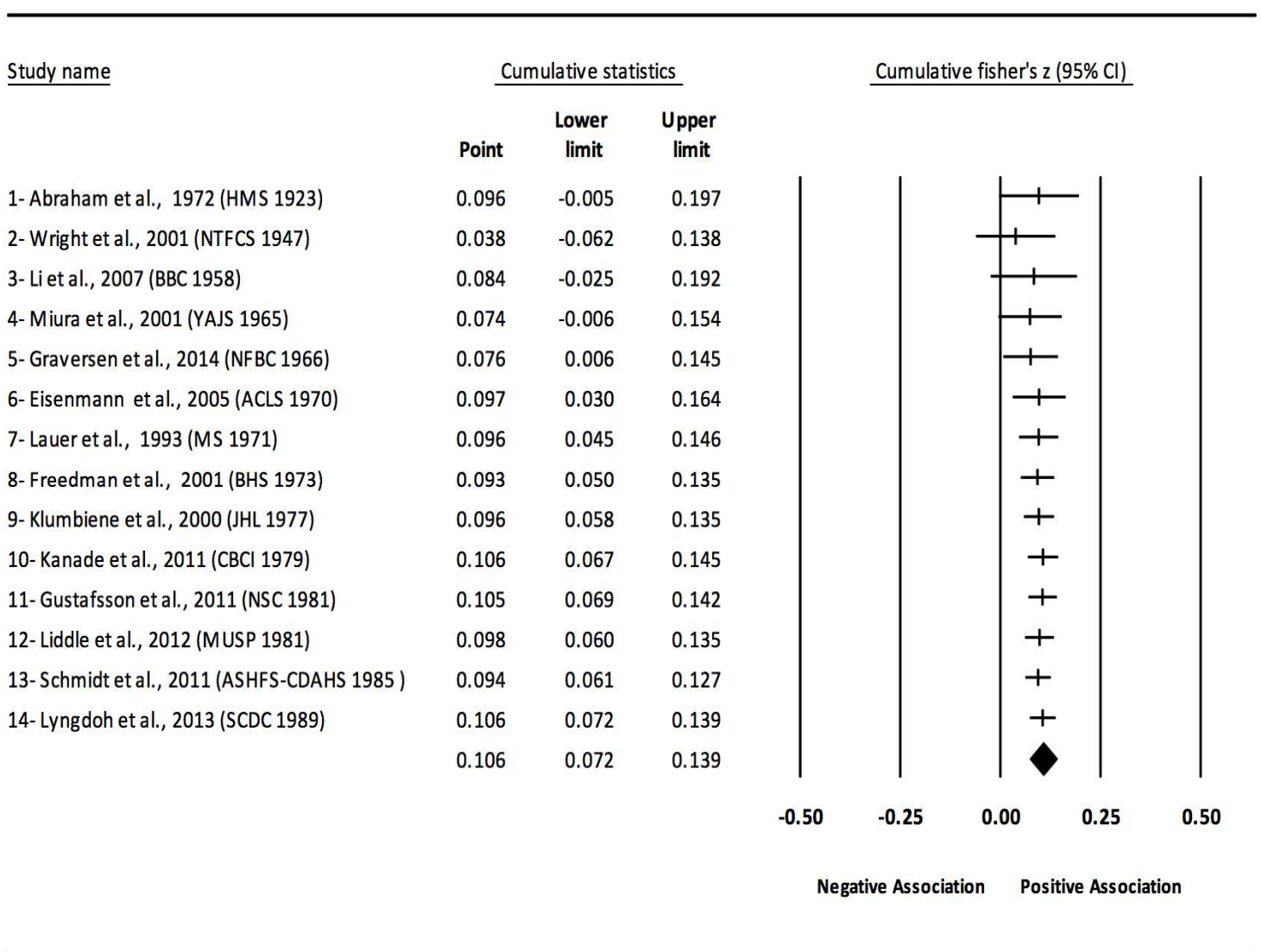

Cumulative meta-analysis, ordered by the year the study started. The vertical lines represent the represent the Fishers $\mathrm{Zr}$ while the left and right extremes of the vertical lines represent the corresponding $95 \%$ confidence intervals. The results of each corresponding study are pooled with all studies preceding it. The middle of the black diamond represents the overall Fishers $\mathrm{Zr}$ while the left and right extremes of the diamond represent the corresponding 95\% confidence intervals. Combined measures represent those studies in which male and female were combined, or different age cohorts from each study were combines, or multiple readings from the same cohort were combined or one study using more than one exposure definition. 
Figure 2b. Cumulative meta-analysis for the association between childhood obesity and adult DBP adjusted for adult BMI

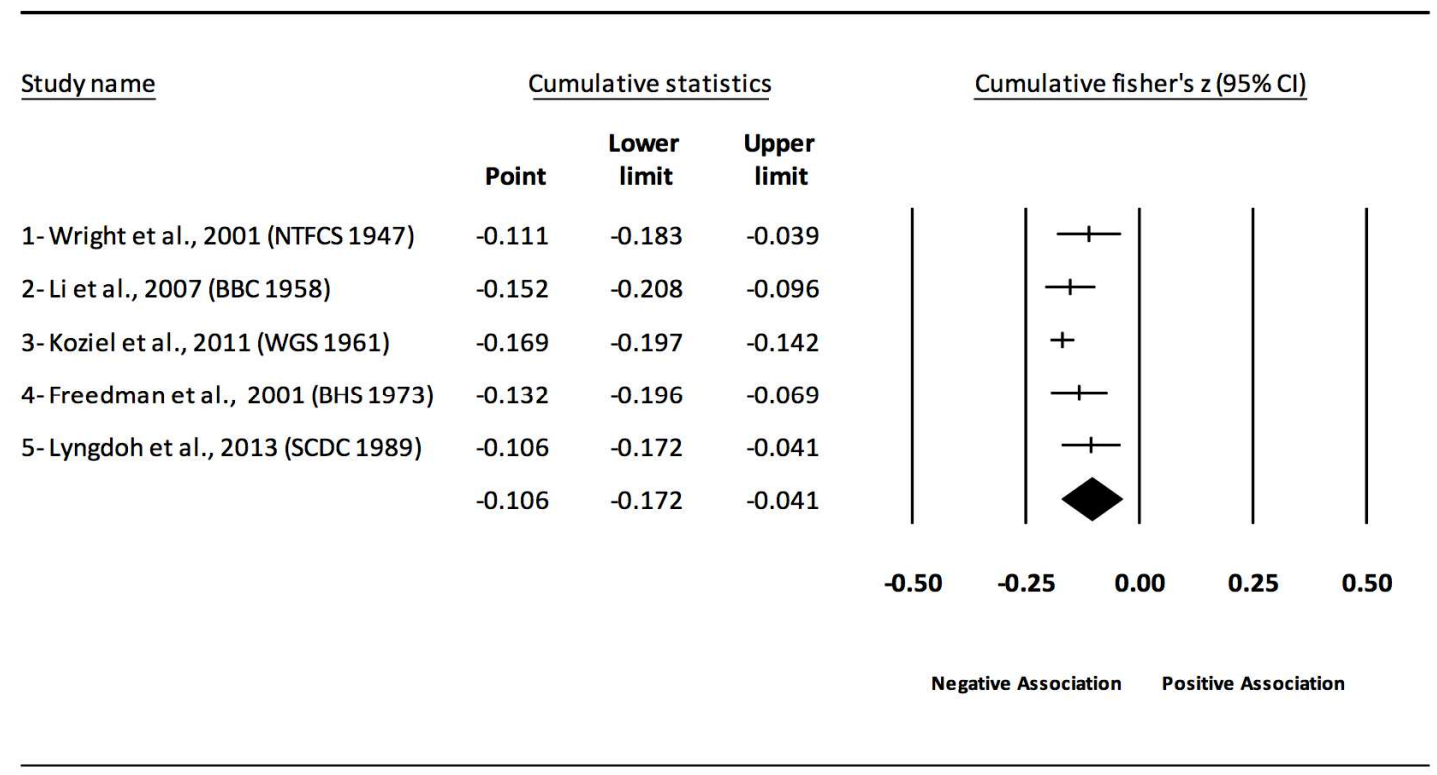

Cumulative meta-analysis, ordered by the year the study started. The vertical lines represent the represent the Fishers $\mathrm{Zr}$ while the left and right extremes of the vertical lines represent the corresponding $95 \%$ confidence intervals. The results of each corresponding study are pooled with all studies preceding it. The middle of the black diamond represents the overall Fishers $\mathrm{Zr}$ while the left and right extremes of the diamond represent the corresponding $95 \%$ confidence intervals. Combined measures represent those studies in which male and female were combined, or different age cohorts from each study were combines, or multiple readings from the same cohort were combined or one study using more than one exposure definition. 
Figure 3a. Cumulative meta-analysis for the association between childhood obesity and adult TC

\begin{tabular}{|c|c|c|c|c|c|c|c|c|}
\hline \multirow[t]{2}{*}{ Study name } & \multicolumn{3}{|c|}{ Cumulative statistics } & \multicolumn{5}{|c|}{ Cumulative fisher's z $(95 \% \mathrm{CI})$} \\
\hline & Point & $\begin{array}{c}\text { Lower } \\
\text { limit }\end{array}$ & $\begin{array}{c}\text { Upper } \\
\text { limit }\end{array}$ & & & & & \\
\hline 1- Abraham et al., 1973 (HMS 1923) & -0.006 & -0.109 & 0.097 & & & $\longrightarrow$ & & \\
\hline 2- Skidmore et al., 2007 (NSHD 1946) & -0.040 & -0.059 & -0.021 & & & + & & \\
\hline 3- Wright et al., 2001 (NTFCS 1947) & -0.043 & -0.062 & -0.025 & & & + & & \\
\hline 4- Miura et al., 2001 (YAJS 1965) & -0.023 & -0.076 & 0.030 & & & - & & \\
\hline 5- Weitz et al., 2014 (LS-Mid-TC 1966) & -0.016 & -0.065 & 0.032 & & & - & & \\
\hline 6- Eisenmann et al., 2005 (ACLS 1970) & -0.017 & -0.063 & 0.028 & & & & & \\
\hline 7- Freedman et al., 2001 (BHS 1973) & 0.004 & -0.056 & 0.064 & & & & & \\
\hline \multirow[t]{4}{*}{ 8-Porkka et al., 1994 (YFS 1980) } & 0.008 & -0.048 & 0.064 & & & & & \\
\hline & 0.008 & -0.048 & 0.064 & & & & & \\
\hline & & & & -0.50 & -0.25 & 0.00 & 0.25 & 0.50 \\
\hline & & & & \multicolumn{5}{|c|}{ Negative Association Positive Association } \\
\hline
\end{tabular}

Cumulative meta-analysis, ordered by the year the study started. The vertical lines represent the represent the Fishers $\mathrm{Zr}$ while the left and right extremes of the vertical lines represent the corresponding $95 \%$ confidence intervals. The results of each corresponding study are pooled with all studies preceding it. The middle of the black diamond represents the overall Fishers $\mathrm{Zr}$ while the left and right extremes of the diamond represent the corresponding 95\% confidence intervals. Combined measures represent those studies in which male and female were combined, or different age cohorts from each study were combines, or multiple readings from the same cohort were combined or one study using more than one exposure definition. 
Figure 3b. Cumulative meta-analysis for the association between childhood obesity and adult TC adjusted for adult BMI

\begin{tabular}{|c|c|c|c|c|c|c|c|c|}
\hline \multirow[t]{3}{*}{ Study name } & \multicolumn{3}{|c|}{ Cumulative statistics } & \multicolumn{5}{|c|}{ Cumulative fisher's z $(95 \% \mathrm{Cl})$} \\
\hline & \multicolumn{3}{|c|}{ Lower Upper } & & & & & \\
\hline & Point & limit & limit & & & & & \\
\hline 1- Barker et al., 2005 (HBCS 1934) & 0.024 & -0.022 & 0.070 & & & + & & \\
\hline 2 Wright et al., 2001 (NTFCS 1947) & -0.009 & -0.085 & 0.066 & & & & & \\
\hline 3- Koziel et al., 2011 (WGS 1961) & -0.049 & -0.143 & 0.045 & & & & & \\
\hline \multirow[t]{4}{*}{ 4- Freedman et al., 2001 (BHS 1973) } & -0.057 & -0.120 & 0.005 & & & & & \\
\hline & -0.057 & -0.120 & 0.005 & & & & & \\
\hline & & & & -0.50 & -0.25 & 0.00 & 0.25 & 0.50 \\
\hline & & & & \multicolumn{5}{|c|}{ Negative Association Positive Association } \\
\hline
\end{tabular}

Cumulative meta-analysis, ordered by the year the study started. The vertical lines represent the represent the Fishers $\mathrm{Zr}$ while the left and right extremes of the vertical lines represent the corresponding $95 \%$ confidence intervals. The results of each corresponding study are pooled with all studies preceding it. The middle of the black diamond represents the overall Fishers Zr while the left and right extremes of the diamond represent the corresponding 95\% confidence intervals. Combined measures represent those studies in which male and female were combined, or different age cohorts from each study were combines, or multiple readings from the same cohort were combined or one study using more than one exposure definition. 
Figure 4a. Cumulative meta-analysis for the association between childhood obesity and adult LDL

\begin{tabular}{|c|c|c|c|c|c|c|c|}
\hline \multirow[t]{2}{*}{ Study name } & \multicolumn{3}{|c|}{ Cumulative statistics } & \multicolumn{4}{|c|}{ Cumulative fisher's z $(95 \% \mathrm{Cl})$} \\
\hline & Point & $\begin{array}{l}\text { Lower } \\
\text { limit }\end{array}$ & $\begin{array}{l}\text { Upper } \\
\text { limit }\end{array}$ & & & & \\
\hline 1- Skidmore et al., 2007 (NSHD 1946) & -0.029 & -0.049 & -0.009 & & & + & \\
\hline 2- Wright et al., 2001 (NTFCS 1947) & -0.046 & -0.097 & 0.005 & & & - & \\
\hline 3- Freedman et al., 2001 (BHS 1973) & -0.003 & -0.102 & 0.097 & & & & \\
\hline 4- Porkka et al., 1994 (YFS 1980) & 0.004 & -0.082 & 0.090 & & & & \\
\hline \multirow[t]{4}{*}{ 5- Lyngdoh et al., 2013 (SCDC 1989) } & 0.023 & -0.055 & 0.101 & & & & \\
\hline & 0.023 & -0.055 & 0.101 & & & & \\
\hline & & & & -0.50 & -0.25 & 0.25 & 0.50 \\
\hline & & & & \multicolumn{4}{|c|}{ Negative Association Positive Association } \\
\hline
\end{tabular}

Cumulative meta-analysis, ordered by the year the study started. The vertical lines represent the represent the Fishers $\mathrm{Zr}$ while the left and right extremes of the vertical lines represent the corresponding $95 \%$ confidence intervals. The results of each corresponding study are pooled with all studies preceding it. The middle of the black diamond represents the overall Fishers $\mathrm{Zr}$ while the left and right extremes of the diamond represent the corresponding $95 \%$ confidence intervals. Combined measures represent those studies in which male and female were combined, or different age cohorts from each study were combines, or multiple readings from the same cohort were combined or one study using more than one exposure definition. 
Figure 4b. Cumulative meta-analysis for the association between childhood obesity and adult LDL adjusted for adult BMI

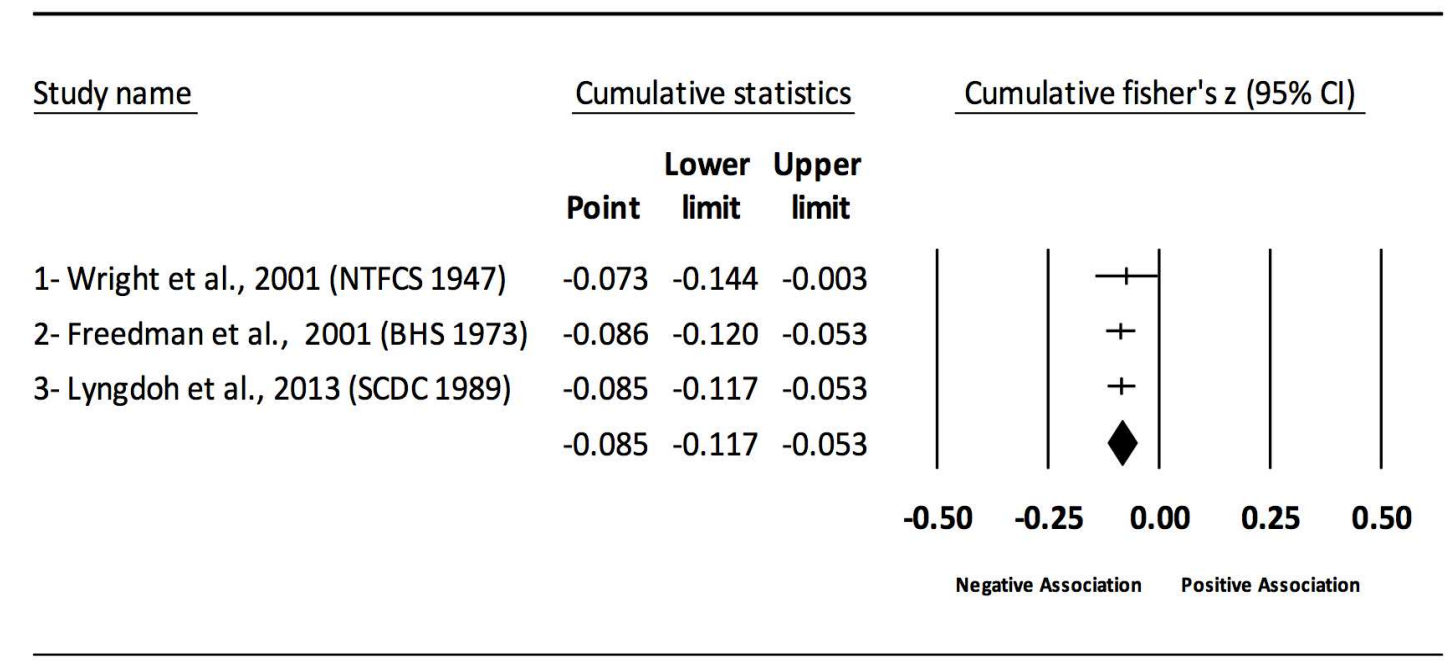

Cumulative meta-analysis, ordered by the year the study started. The vertical lines represent the represent the Fishers $\mathrm{Zr}$ while the left and right extremes of the vertical lines represent the corresponding $95 \%$ confidence intervals. The results of each corresponding study are pooled with all studies preceding it. The middle of the black diamond represents the overall Fishers $\mathrm{Zr}$ while the left and right extremes of the diamond represent the corresponding 95\% confidence intervals. Combined measures represent those studies in which male and female were combined, or different age cohorts from each study were combines, or multiple readings from the same cohort were combined or one study using more than one exposure definition. 
Figure 5a. Cumulative meta-analysis for the association between childhood obesity and adult HDL

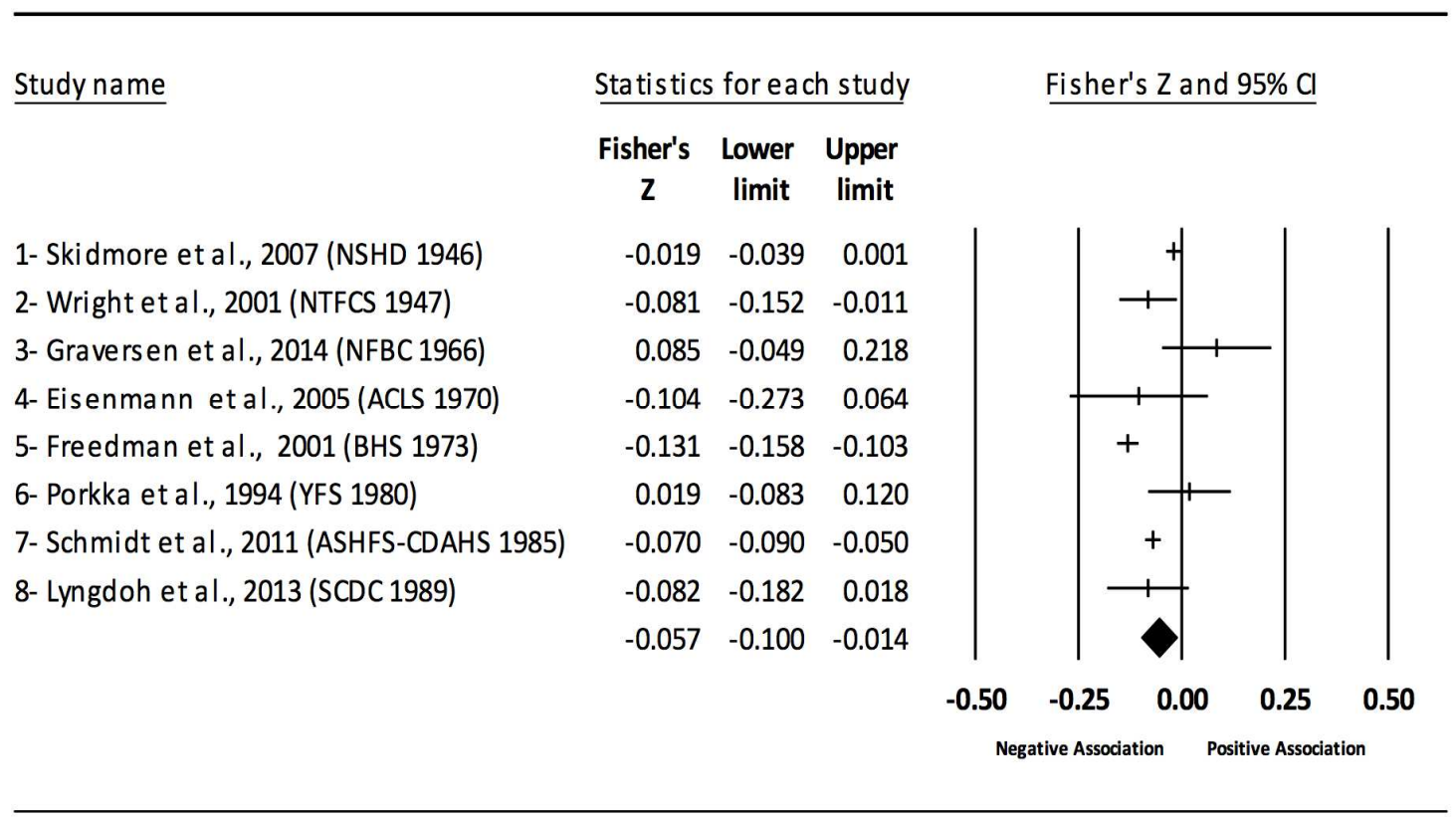

Cumulative meta-analysis, ordered by the year the study started. The vertical lines represent the represent the Fishers $\mathrm{Zr}$ while the left and right extremes of the vertical lines represent the corresponding $95 \%$ confidence intervals. The results of each corresponding study are pooled with all studies preceding it. The middle of the black diamond represents the overall Fishers $\mathrm{Zr}$ while the left and right extremes of the diamond represent the corresponding 95\% confidence intervals. Combined measures represent those studies in which male and female were combined, or different age cohorts from each study were combines, or multiple readings from the same cohort were combined or one study using more than one exposure definition. 
Figure 5b. Cumulative meta-analysis for the association between childhood obesity and adult HDL adjusted for adult BMI

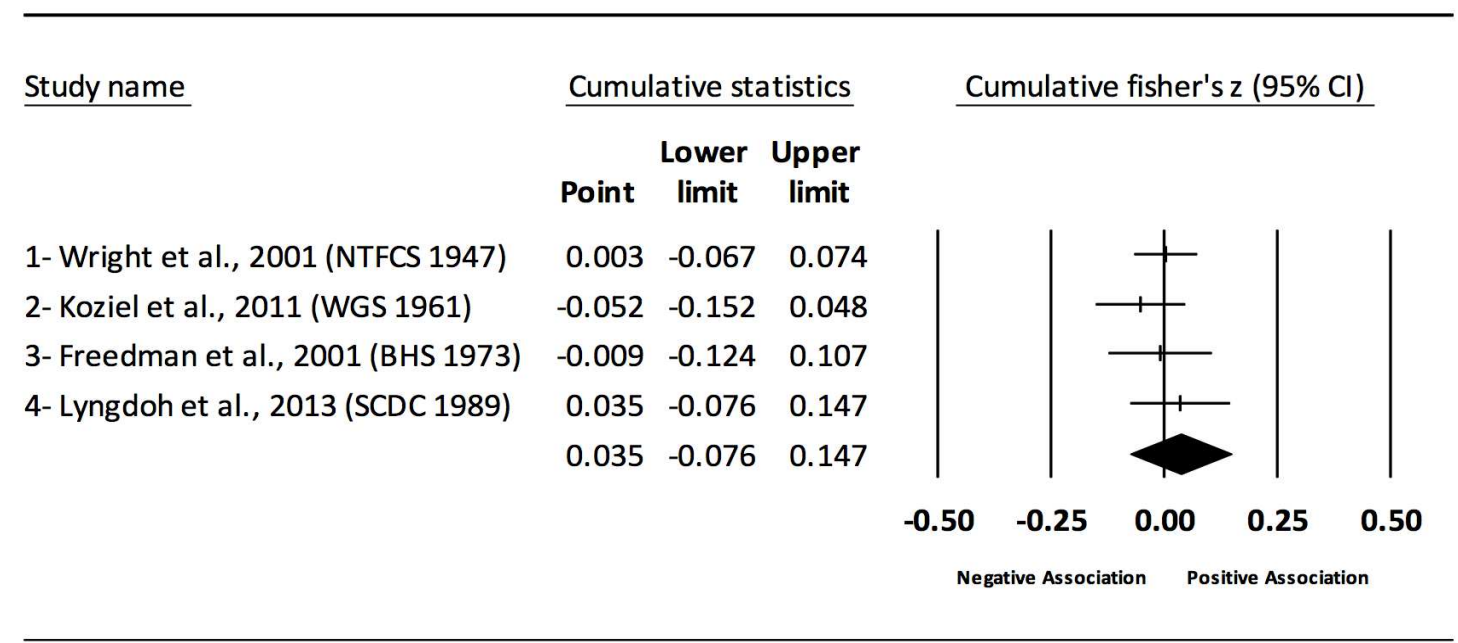

Cumulative meta-analysis, ordered by the year the study started. The vertical lines represent the represent the Fishers $\mathrm{Zr}$ while the left and right extremes of the vertical lines represent the corresponding $95 \%$ confidence intervals. The results of each corresponding study are pooled with all studies preceding it. The middle of the black diamond represents the overall Fishers $\mathrm{Zr}$ while the left and right extremes of the diamond represent the corresponding $95 \%$ confidence intervals. Combined measures represent those studies in which male and female were combined, or different age cohorts from each study were combines, or multiple readings from the same cohort were combined or one study using more than one exposure definition. 
Figure 6a. Cumulative meta-analysis for the association between childhood obesity and adult TG

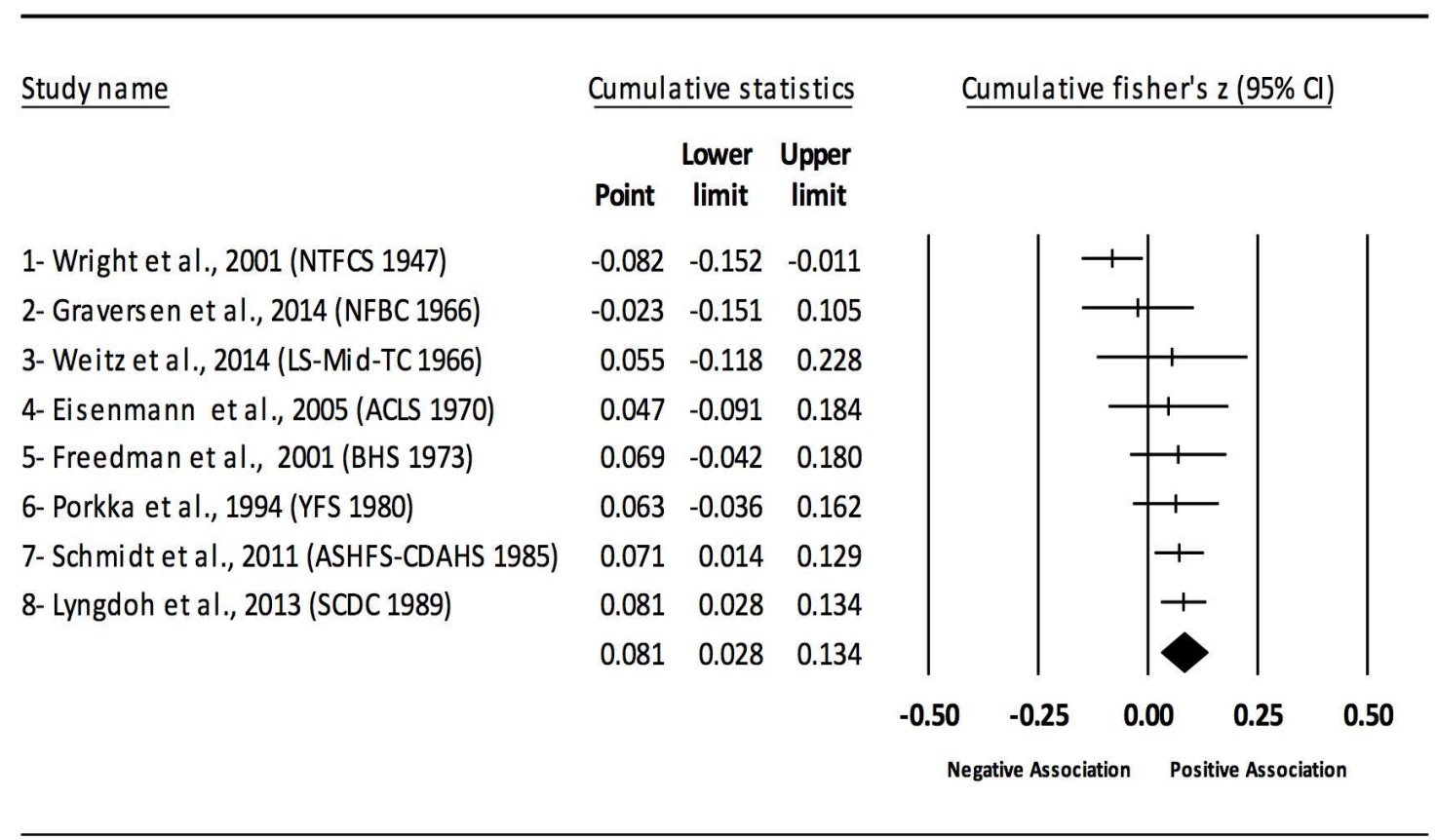

Cumulative meta-analysis, ordered by the year the study started. The vertical lines represent the represent the Fishers $\mathrm{Zr}$ while the left and right extremes of the vertical lines represent the corresponding $95 \%$ confidence intervals. The results of each corresponding study are pooled with all studies preceding it. The middle of the black diamond represents the overall Fishers $\mathrm{Zr}$ while the left and right extremes of the diamond represent the corresponding $95 \%$ confidence intervals. Combined measures represent those studies in which male and female were combined, or different age cohorts from each study were combines, or multiple readings from the same cohort were combined or one study using more than one exposure definition. 
Figure 6b. Cumulative meta-analysis for the association between childhood obesity and adult TG

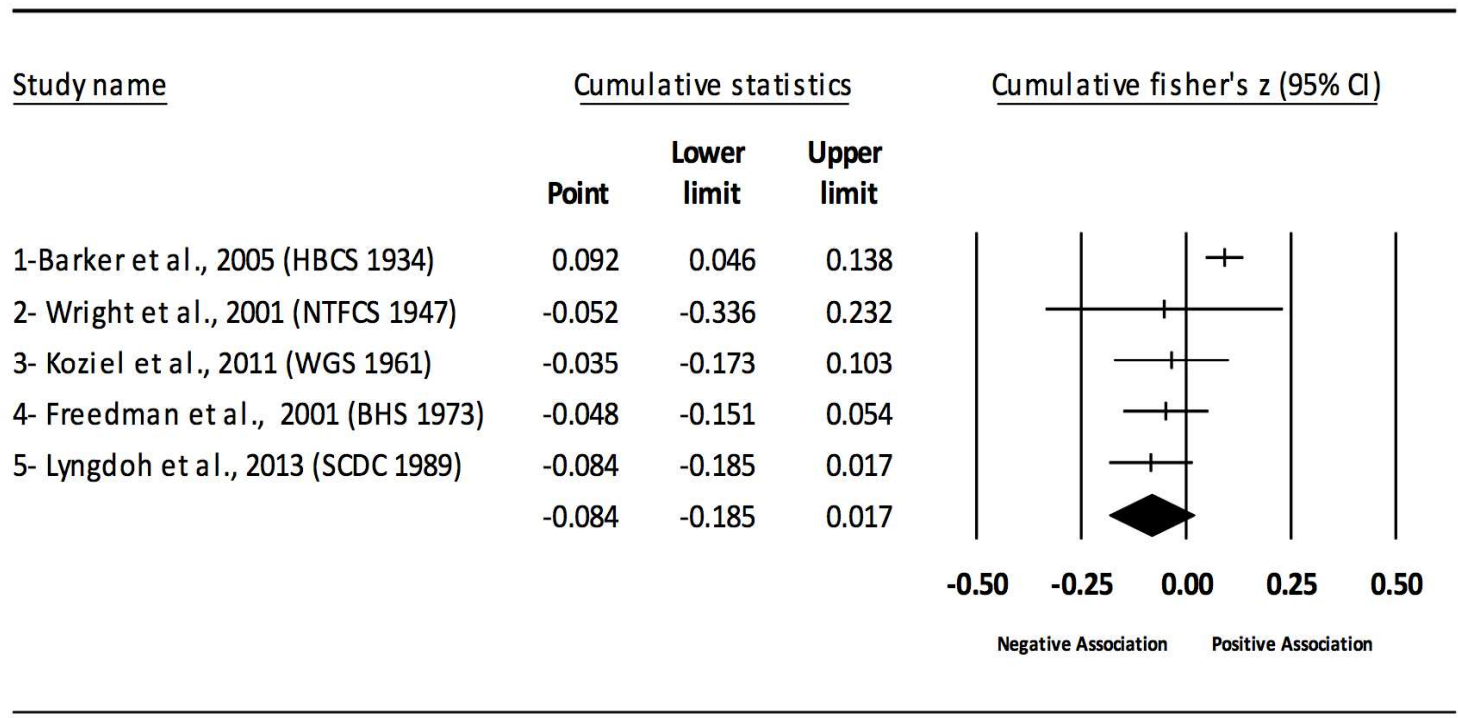

Cumulative meta-analysis, ordered by the year the study started. The vertical lines represent the represent the Fishers $\mathrm{Zr}$ while the left and right extremes of the vertical lines represent the corresponding $95 \%$ confidence intervals. The results of each corresponding study are pooled with all studies preceding it. The middle of the black diamond represents the overall Fishers Zr while the left and right extremes of the diamond represent the corresponding $95 \%$ confidence intervals. Combined measures represent those studies in which male and female were combined, or different age cohorts from each study were combines, or multiple readings from the same cohort were combined or one study using more than one exposure definition. 
Funnel plots:

Figure 1: Funnel plot for the association between childhood obesity and adult SBP

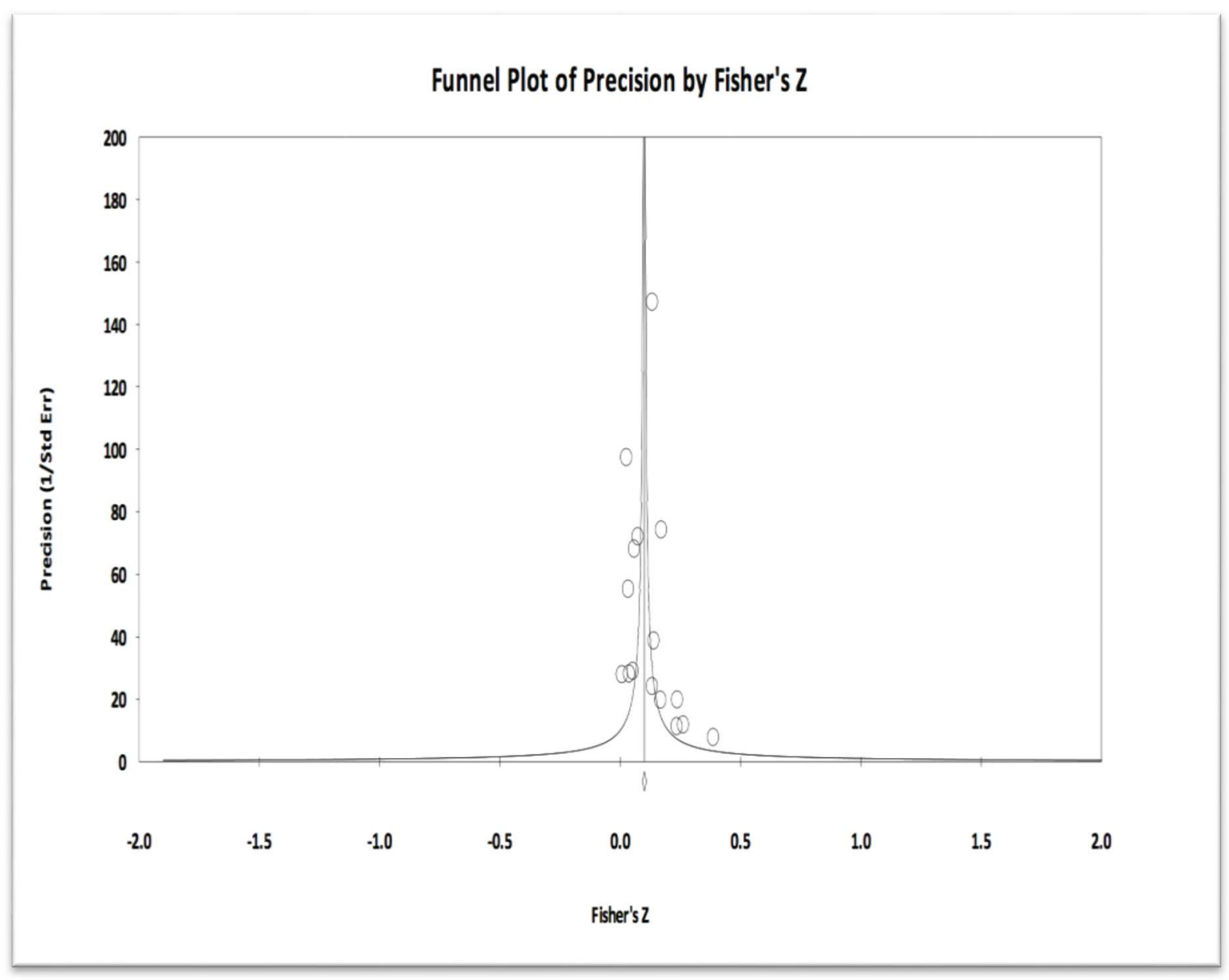


Figure 1: Funnel plot for the association between childhood obesity and adult DBP

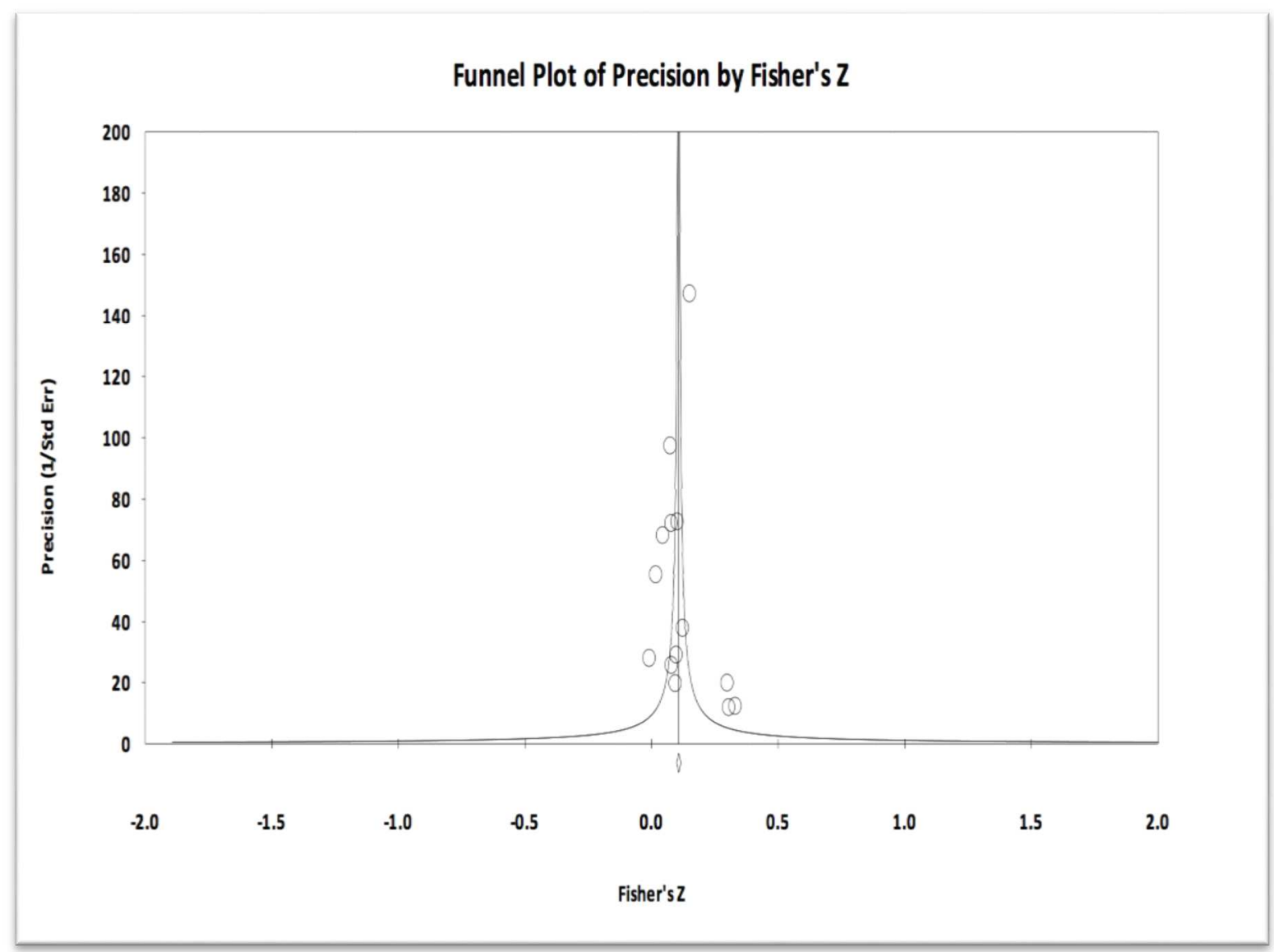




\section{Additional File 5}

Meta-Regression results for the association between childhood adiposity and adult cardiovascular disease (CVD) risk factors

Table 1: Systolic Blood Pressure (SBP) and Diastolic Blood Pressure (DBP)

\begin{tabular}{|c|c|c|c|c|c|c|c|c|}
\hline \multicolumn{9}{|c|}{ CVD risk factors } \\
\hline \multirow[b]{2}{*}{ Covariate } & \multicolumn{4}{|c|}{ SBP } & \multicolumn{4}{|c|}{ DBP } \\
\hline & $\begin{array}{c}\text { \# of } \\
\text { Studies }\end{array}$ & Beta \pm SE & CI $(95 \%)$ & P-value & $\begin{array}{c}\text { \# of } \\
\text { Studies } \\
\end{array}$ & Beta \pm SE & CI $(95 \%)$ & $\begin{array}{c}\text { P- } \\
\text { value }\end{array}$ \\
\hline Baseline Age & 16 & $0.0104 \pm 0.004$ & $0.0025,0.0183$ & 0.0096 & 14 & $0.0096 \pm 0.0039$ & $0.0019,0.0173$ & 0.0145 \\
\hline Follow-up Age & 16 & $-0.0006 \pm 0.002$ & $-0.0045,0.0033$ & 0.7559 & 14 & $-0.0011 \pm 0.0016$ & $-0.0042,0.0021$ & 0.5116 \\
\hline Length of FU & 16 & $-0.0024 \pm 0.002$ & $-0.0063,0.0014$ & 0.216 & 14 & $-0.0028 \pm 0.0017$ & $-0.0062,0.0006$ & 0.1076 \\
\hline Type of Analysis & 16 & $0.0218 \pm 0.0421$ & $-0.0607,0.1043$ & 0.6046 & 14 & $0.0176 \pm 0.0393$ & $-0.0594,0.0946$ & 0.6535 \\
\hline Country & 16 & $0.0741 \pm 0.0386$ & $-0.0015,0.1497$ & 0.0548 & 14 & $0.014 \pm 0.0412$ & $-0.0668,0.0948$ & 0.7342 \\
\hline$*$ Sex & & $0.0303 \pm 0.029$ & $-0.0265,0.0871$ & 0.2964 & & $-0.0304 \pm 0.0294$ & $-0.0879,0.0272$ & 0.3008 \\
\hline \multirow[t]{3}{*}{ *Exposure } & & $0.0476 \pm 0.0245$ & $-0.0005,0.0956$ & 0.0524 & & $0.0156 \pm 0.0258$ & $-0.0351,0.0662$ & 0.5465 \\
\hline & \multicolumn{7}{|c|}{ CVD risk factors adjusted for adult BMI } & \\
\hline & \multicolumn{4}{|c|}{ SBP } & \multicolumn{4}{|c|}{ DBP } \\
\hline Baseline Age & 6 & $0.0055 \pm 0.0084$ & $-0.011,0.0221$ & 0.5118 & 5 & $0.0072 \pm 0.0249$ & $-0.0416,0.0559$ & 0.7723 \\
\hline Follow-up Age & 6 & $-0.0046 \pm 0.0015$ & $-0.0075,-0.0017$ & 0.0017 & 5 & $-0.006 \pm 0.0014$ & $-0.0087,-0.0032$ & 0.0001 \\
\hline Follow-up Time & 6 & $-0.0038 \pm 0.0014$ & $-0.0066,-0.001$ & 0.0078 & 5 & $-0.0061 \pm 0.0014$ & $-0.0089,-0.0034$ & 0.0001 \\
\hline$*$ Sex & & $0.0409 \pm 0.0432$ & $-0.0438,0.1256$ & 0.3435 & & $0.0165 \pm 0.045$ & $-0.0717,0.1047$ & 0.7141 \\
\hline \multicolumn{9}{|c|}{$\begin{array}{l}\text { Type of Analysis: Correlation/Beta coefficient vs. Mean Difference/OR/RR } \\
\text { Country: USA vs. Others } \\
\text { Sex: Male vs. Female } \\
\text { Exposure: BMI vs. Other Measures of Adiposity } \\
\text { *Factors assessed using sub-group as the study of analysis instead of study as the unit of analysis }\end{array}$} \\
\hline For categorical val & les. less & an three recults for & - & & & & & \\
\hline
\end{tabular}


Table 3: High-density lipoprotein cholesterol (HDL) and triglycerides (TG)

CVD risk factors

\begin{tabular}{|c|c|c|c|c|c|c|c|c|}
\hline \multirow[b]{2}{*}{ Covariate } & \multicolumn{4}{|c|}{ HDL } & \multicolumn{4}{|c|}{ TG } \\
\hline & $\begin{array}{c}\text { \# of } \\
\text { Studies }\end{array}$ & Beta \pm SE & CI $(95 \%)$ & P-value & $\begin{array}{c}\text { \# of } \\
\text { Studies }\end{array}$ & Beta $\pm \mathrm{SE}$ & CI $(95 \%)$ & $\begin{array}{c}\text { P- } \\
\text { value }\end{array}$ \\
\hline Baseline Age & 8 & $-0.0072 \pm 0.0079$ & $-0.0227,0.0083$ & 0.3622 & 8 & $-0.0041 \pm 0.0095$ & $-0.0227,0.0146$ & 0.6699 \\
\hline Follow-up Age & 8 & $0.0014 \pm 0.0016$ & $-0.0017,0.0045$ & 0.3754 & 8 & $-0.0091 \pm 0.0016$ & $-0.0122,-0.006$ & 0.0001 \\
\hline Length of FU & 8 & $0.0013 \pm 0.0014$ & $-0.0015,0.0041$ & 0.3507 & 8 & $-0.0073 \pm 0.0019$ & $-0.011,-0.0037$ & 0.0001 \\
\hline Type of Analysis & & & & & & & & \\
\hline Country & & & & & & & & \\
\hline$*$ Sex & & $0.0144 \pm 0.0517$ & $-0.0868,0.1157$ & 0.7798 & & $0.0141 \pm 0.0964$ & $-0.1749,0.2031$ & 0.884 \\
\hline *Exposure & & $0.0231 \pm 0.0232$ & $-0.0224,0.0685$ & 0.3197 & & $-0.0237 \pm 0.0329$ & $-0.0882,0.0408$ & 0.4715 \\
\hline
\end{tabular}

CVD risk factors adjusted for adult BMI

HDL

TG

\begin{tabular}{|c|c|c|c|c|c|c|c|c|}
\hline Baseline Age & 4 & $0.0104 \pm 0.0438$ & $-0.0755,0.0963$ & 0.8123 & 5 & $-0.0208 \pm 0.0112$ & $-0.0427,0.0011$ & 0.0622 \\
\hline Follow-up Age & 4 & $-0.0069 \pm 0.0024$ & $-0.0115,-0.0022$ & 0.0036 & 5 & $0.0055 \pm 0.0028$ & $0,0.0109$ & 0.0493 \\
\hline Length of FU & 4 & $-0.0068 \pm 0.0024$ & $-0.0115,-0.0022$ & 0.0041 & 5 & $0.0049 \pm 0.0021$ & $0.0008,0.0091$ & 0.0205 \\
\hline$*$ Sex & & $-0.1345 \pm 0.0528$ & $-0.2381,-0.031$ & 0.0109 & & $0.144 \pm 0.0456$ & $0.0546,0.2334$ & 0.0016 \\
\hline
\end{tabular}

Type of Analysis: Correlation/Beta coefficient vs. Mean Difference/OR/RR

Country: USA vs. Others

Sex: Male vs. Female

Exposure: BMI vs. Other Measures of Adiposity

*Factors assessed using sub-group as the study of analysis instead of study as the unit of analysis

For categorical variables, less than three results for any one category were used as the cut-off for analysis 